\title{
Enhancing the Thermoelectric Performance of Calcium Cobaltite Ca3Co409 through Single and Dual Elements Doping
}

\author{
Cesar Octavio Romo de la Cruz \\ West Virginia University, ceromodelacruz@mix.wvu.edu
}

Follow this and additional works at: https://researchrepository.wvu.edu/etd

Part of the Ceramic Materials Commons

\footnotetext{
Recommended Citation

Romo de la Cruz, Cesar Octavio, "Enhancing the Thermoelectric Performance of Calcium Cobaltite Ca3Co409 through Single and Dual Elements Doping" (2019). Graduate Theses, Dissertations, and Problem Reports. 7396.

https://researchrepository.wvu.edu/etd/7396

This Dissertation is protected by copyright and/or related rights. It has been brought to you by the The Research Repository @ WVU with permission from the rights-holder(s). You are free to use this Dissertation in any way that is permitted by the copyright and related rights legislation that applies to your use. For other uses you must obtain permission from the rights-holder(s) directly, unless additional rights are indicated by a Creative Commons license in the record and/ or on the work itself. This Dissertation has been accepted for inclusion in WVU Graduate Theses, Dissertations, and Problem Reports collection by an authorized administrator of The Research Repository @ WVU. For more information, please contact researchrepository@mail.wvu.edu.
} 
Enhancing the Thermoelectric Performance of Calcium Cobaltite Ca3Co409 through Single and Dual Elements Doping

Cesar Octavio Romo de la Cruz

Follow this and additional works at: https://researchrepository.wvu.edu/etd

Part of the Ceramic Materials Commons 


\title{
Enhancing the Thermoelectric Performance of Calcium Cobaltite through Single and Dual Elements Doping
}

\author{
Cesar Octavio Romo de la Cruz
}

\author{
Dissertation \\ Submitted to the Benjamin M. Statler College of Engineering and Mineral Resources \\ at West Virginia University \\ in partial fulfillment of the requirements for the degree of \\ Doctor of Philosophy in \\ Mechanical Engineering \\ Xueyan Song, Ph.D., Chair \\ Yun Chen, Ph. D. \\ Hailin Li, Ph.D. \\ Jacky Prucz, Ph. D. \\ David Tucker, Ph.D. \\ Department of Mechanical and Aerospace Engineering \\ Morgantown, West Virginia \\ 2019
}

Keywords: Calcium Cobalt Oxide, thermoelectric materials, grain boundaries, nanostructure Copyright 2019 Cesar Octavio Romo de la Cruz 


\section{Abstract \\ Enhancing the Thermoelectric Performance of Calcium Cobaltite $\mathrm{Ca}_{3} \mathrm{Co}_{4} \mathrm{O}_{9}$ through Single and Dual Elements Doping}

\section{Cesar Octavio Romo de la Cruz}

Worldwide energy consumption losses more than $60 \%$ of the energy after its conversion, most of these losses are in the form of waste heat. Therefore, it is a matter of utmost importance to harness waste heat and reutilize this energy source to improve the overall conversion efficiency. Thermoelectric (TE) technology is one of the rising alternatives to harvest the excessive amount of energy lost as waste heat for a better and sustainable energy future. TE materials can utilize waste heat to improve efficiency in the ever-increasing demand for energy by converting a temperature difference directly into electricity due to the Seebeck effect. The energy conversion efficiency of TE materials is described by the figure of merit $Z T$, which is defined as $Z T=S^{2} \rho^{-1} \kappa^{-1} T$, where $S, \rho, S^{2} \rho^{-1}$, and $\kappa$ are the Seebeck coefficient, electrical resistivity, electrical power factor(PF) and thermal conductivity, respectively. P-type calcium cobaltite $\mathrm{Ca}_{3} \mathrm{Co}_{4} \mathrm{O}_{9-\delta}$ is a promising candidate for TE applications over conventional TE materials due to its high thermal stability in the air at high temperatures, low cost, lightweight, and non-toxicity. Single crystal $\mathrm{Ca}_{3} \mathrm{Co}_{4} \mathrm{O}_{9-\delta}$ already possess an excellent TE performance, approaching that of the well-developed conventional TE materials. However, TE energy conversion efficiency of polycrystalline $\mathrm{Ca}_{3} \mathrm{Co}_{4} \mathrm{O}_{9-\delta}$ remains low and accounts to $\sim 30-60 \%$ of the single crystals. To overcome the low performance of the $\mathrm{ZT}$ in $\mathrm{Ca}_{3} \mathrm{Co}_{4} \mathrm{O}_{9-\delta}$ ceramics, dopants were introduced through both the cation stoichiometric substitution and novel non-stoichiometric addition. This thesis work shows that dopants intragranular non-stoichiometric addition and concurrent intergranular segregation at the grain boundaries dramatically decrease the electrical resistivity, simultaneously increase the Seebeck coefficient, and ultimately result in the polycrystalline ceramics outperforming the single crystals over a wide range of temperatures.

A record high thermoelectric figure of merit ZT 0.9 at $1073 \mathrm{~K}$ was achieved for $\mathrm{Ca}_{3} \mathrm{Co}_{4} \mathrm{O}_{9-\delta}$ ceramics that is surpassing the $\mathrm{Ca}_{3} \mathrm{Co}_{4} \mathrm{O}_{9-\delta}$ single crystals. Furthermore, the engineered $\mathrm{Ca}_{3} \mathrm{Co}_{4} \mathrm{O}_{9-\delta}$ polycrystalline ceramics also outperformed the best reported p-type $\mathrm{SiGe}$ from $373 \mathrm{~K}$ to $973 \mathrm{~K}$, while $\mathrm{Ca}_{3} \mathrm{Co}_{4} \mathrm{O}_{9-\delta}$ ceramics are having just $5-10 \%$ of the cost of $\mathrm{SiGe}$ and will be performing directly in air. Most of all, the present work presents a novel approach to engineer the lattice and grain boundary in ceramics to decouple the strongly coupled thermoelectric parameters of $S, \rho$, and $\kappa$ to largely increase the electrical power factor of thermometric oxide ceramics. 


\section{Acknowledgements}

First and foremost, I would like to thank my advisor Dr. Xueyan Song for her expertise, guidance, encouragement and advice she has provided throughout my time as a $\mathrm{Ph}$. D. student. I have been privileged to have an advisor who gives me the autonomy to explore on my own, and at the same time the leadership and help when I encounter difficulties. Without her help and insights this work would have been an overwhelming assignment. I also want to thank Dr. Yun Chen for his valuable research ideas and recommendations throughout my $\mathrm{Ph}$. D. program.

I would also like to thank the members of my doctoral committee: Dr. Hailin Li, Dr. Jacky Prucz, and Dr. David Tucker for their input, valuable discussions and accessibility. I also want to acknowledge the support of the Benjamin M. Statler College of Engineering and Mineral Resources at West Virginia University.

Many thanks go to all my friends and colleagues at West Virginia University for their valuable support and encouragement, all of them have made my time here such a great experience. Furthermore, completing this work would have been more difficult were if not for the support and friendship provided by the members of Prof. Song's lab research group.

Most importantly, I must express my gratitude to Maria, my wife, who has been patient and loving throughout. For her continuous support and encouragement during all the ups and downs of my research, and for helping me keep my life in proper perspective and balance.

Lastly, I would love to express my sincere and biggest gratitude to my mother, my sister, and my brothers for their love, encouragement, dedication and these many years of support. $\uparrow$ Dad, your belief in me has made this journey possible, thank you. 


\section{Table of Contents}

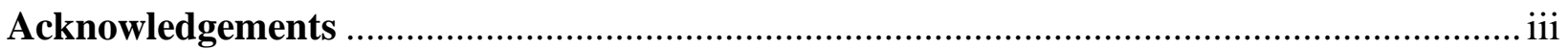

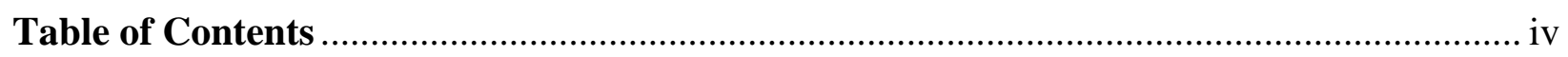

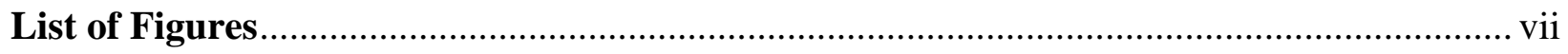

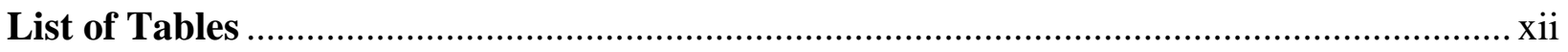

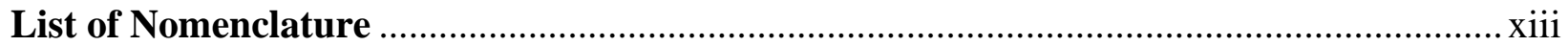

Chapter 1. Introduction to Thermoelectric Materials ....................................................... 1

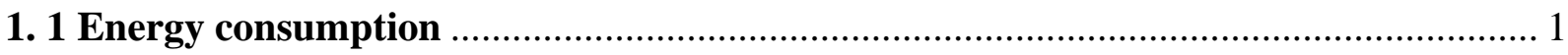

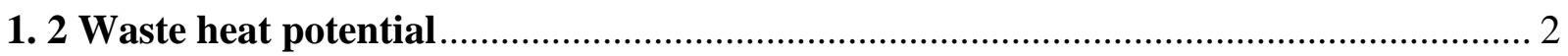

1. 3 Historical background of thermoelectricity .................................................. 4

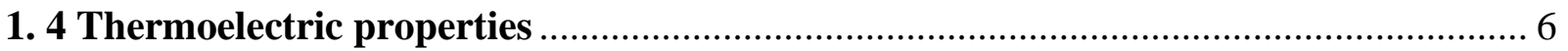

1. 5 Strategies to optimize thermoelectric properties............................................... 11

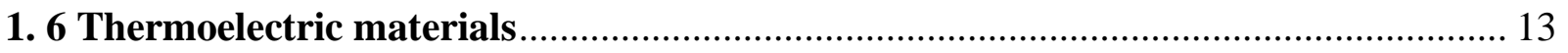

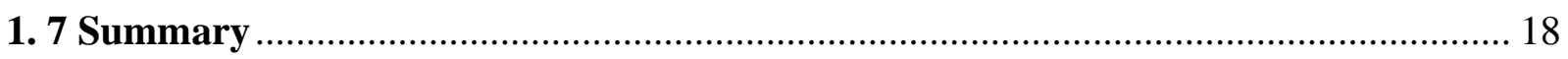

Chapter 2. Review of $\mathrm{Ca}_{3} \mathrm{Co}_{4} \mathrm{O} 9$ Thermoelectric Ceramics .............................................. 23

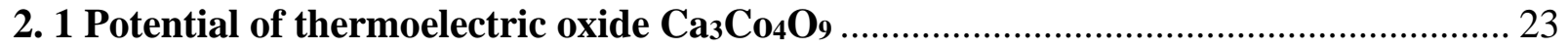

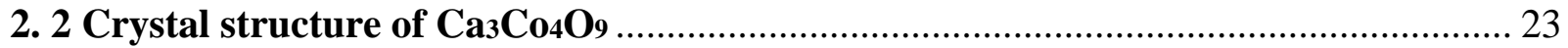

2. 3 Enhancement of $\mathrm{Ca}_{3} \mathrm{Co}_{4} \mathrm{O} 9$ through ion doping and nano-inclusions ....................... 26

2. 4 Enhancement of $\mathrm{Ca}_{3} \mathrm{Co}_{4} \mathrm{O}_{9}$ through modification of the processing techniques....... 36

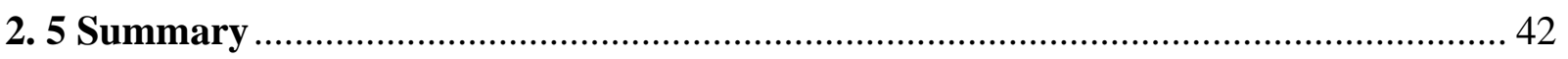

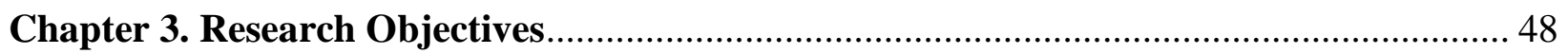

Chapter 4. General Experimental Procedure Utilized in Chapters 5 to 9 ......................... 52

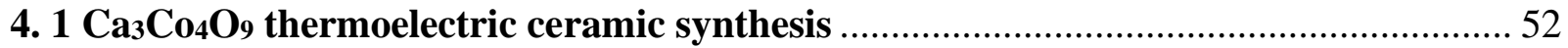

4. 2 Electrical and thermal properties measurement ............................................. 53

4. 3 Microstructure and nanostructure imaging with TEM and SEM ......................... 54

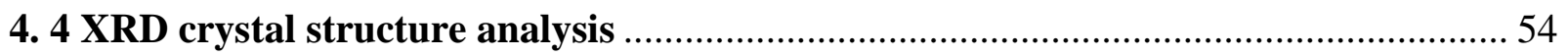

Chapter 5. Impact of Potassium Addition on the Thermoelectric Performance of $\mathrm{Ca}_{3} \mathrm{Co}_{4} \mathrm{O}_{9}$

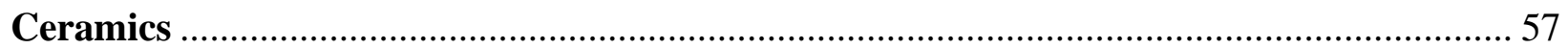

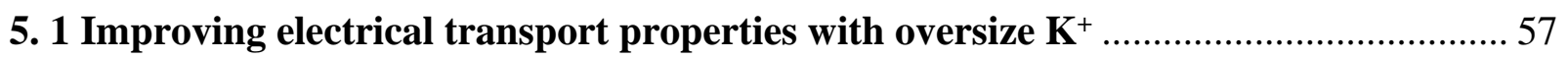

5. 2 Background \& motivation for $\mathrm{Ca}_{3} \mathrm{Co}_{4} \mathrm{O}_{9} \mathrm{~K}_{\mathrm{x}}$ material .......................................... 57

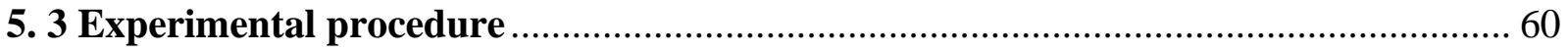

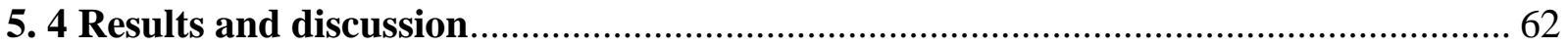




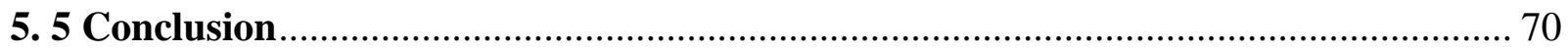

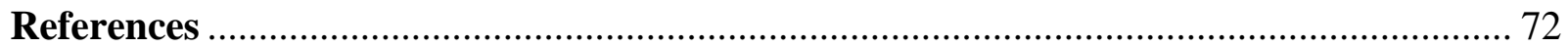

Chapter 6. Influence of the Dual Non-Stoichiometric Addition of Bismuth and Potassium in Thermoelectric Performance of Calcium Cobaltite Ceramics ............................................ 74

6. 1 Complex interactions of dual dopants at GB leading to improved ZT ………........... 74

6. 2 Introduction ............................................................................................................ 75

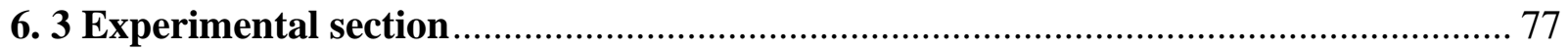

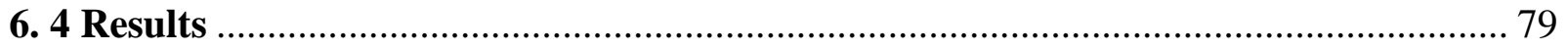

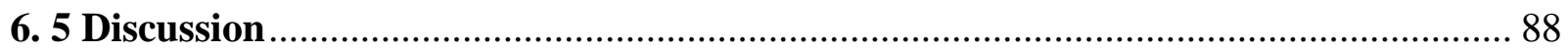

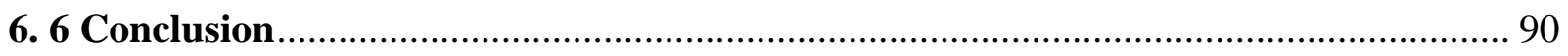

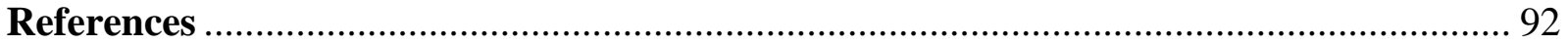

Chapter 7. Influence of Terbium Depletion at the Grain Boundaries on the Nanostructure

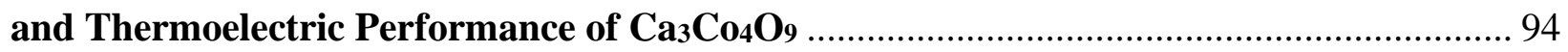

7. 1 Reducing thermal transport properties with Terbium............................................. 94

7. 2 Background \& motivation for $\mathrm{Ca3}_{-\mathbf{x}} \mathrm{Tb}_{\mathbf{x}} \mathrm{Co} \mathrm{C}_{9} \mathrm{O}$ material............................................ 94

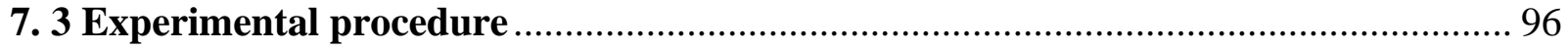

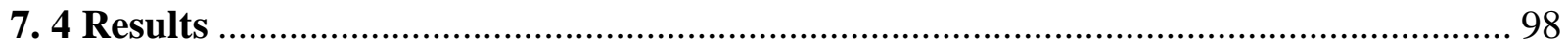

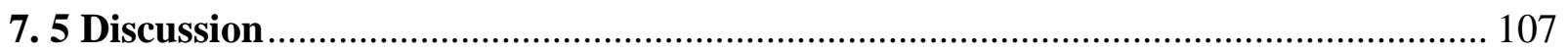

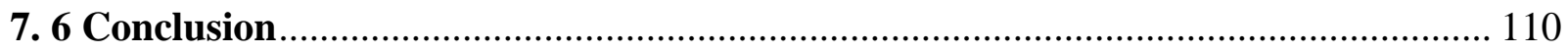

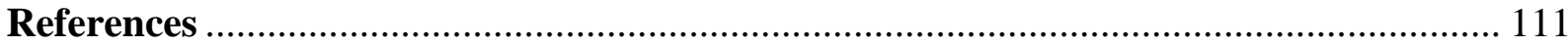

Chapter 8. Impact of Potassium Non-Stoichiometric Addition and Terbium Stoichiometric Substitution on the Thermoelectric Performance of $\mathrm{Ca}_{3} \mathrm{Co}_{4} \mathrm{O}_{9}$ Ceramics …….................. 113

8. 1 Different size of dopants for better ZT performance............................................. 113

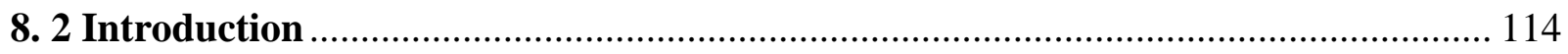

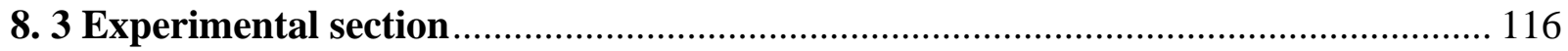

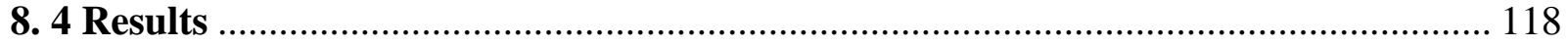

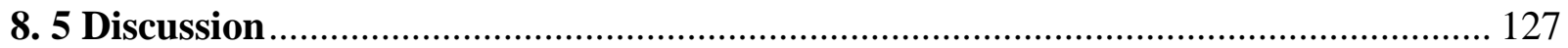

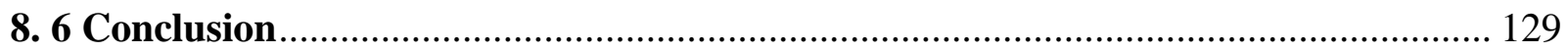

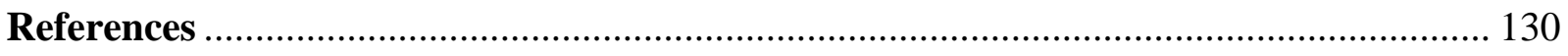

Chapter 9. Systematic Dual Doping Influence on the Thermoelectric Performance of

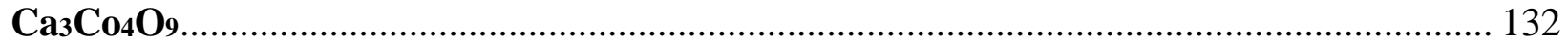

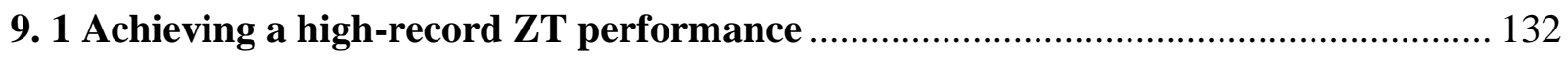

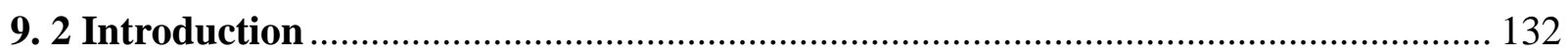




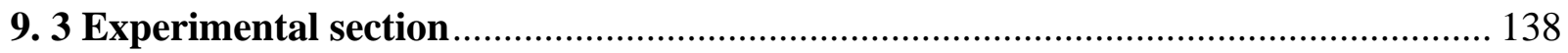

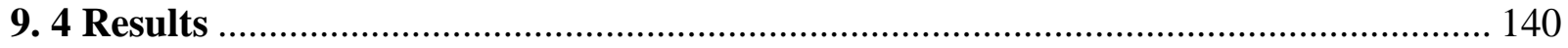

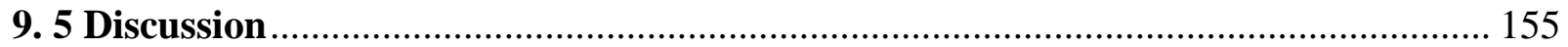

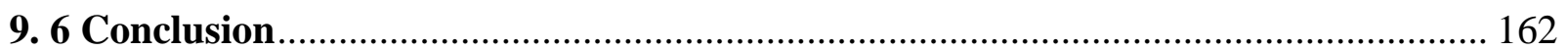

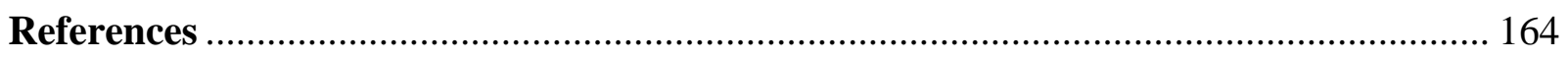

Chapter 10. Conclusion and Future Work ................................................................ 168

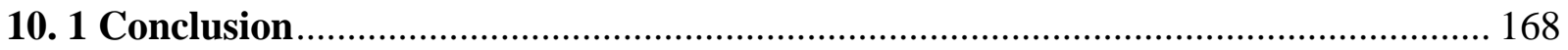

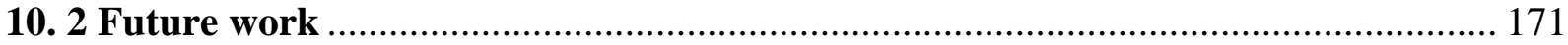

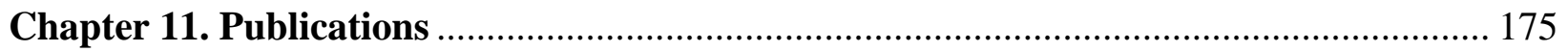




\section{List of Figures}

Figure 1-1: Left: Total primary energy supply by fuel. ${ }^{[1]}$ Mtoe: Million Tons of Oil Equivalent. Right: World energy consumption projections, $1990-2040$ (quadrillion Btu). ${ }^{[2]} \ldots 1$

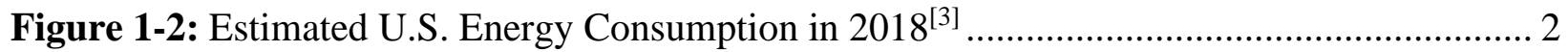

Figure 1-3: A depiction of the thermoelectric effect when a material is subjected to an external temperature gradient and the system is in equilibrium. (a) The more energetic electrons have a longer mean free path. (b) These more energetic electrons (denoted by blue dots) migrate to a lower potential until an electric field (E) is developed to

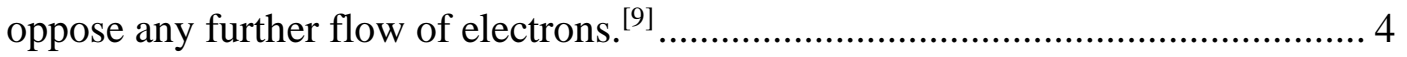

Figure 1-4: Schematic illustrations of a thermoelectric module for (a) power generation (Seebeck effect) and (b) active refrigeration (Peltier effect). ${ }^{[16]}$ 5

Figure 1-5: Thermoelectric module showing the direction of charge flow on power generation. ${ }^{[15]}$

Figure 1-6: Thermoelectric energy conversion as a function of $\mathrm{ZT}$ at the setting of $\mathrm{T}_{\mathrm{c}}=300 \mathrm{~K}$, and efficiency for conventional thermal engines. ${ }^{[28]}$ 8

Figure 1-7: Optimizing ZT through carrier concentration tuning: Left: Maximizing the efficiency (ZT) of a thermoelectric involves finding the middle ground of thermal conductivity and Seebeck coefficient with electrical conductivity. Right: Reducing the lattice thermal conductivity leads to a dual benefit for the thermoelectric figure of merit, lowering the thermal conductivity allows the carrier concentration to be reduced, leading to both a decrease in $\kappa_{\mathrm{el}}$ and a larger $\mathrm{S}^{[15]}$. 11

Figure 1-8: Schematic diagram illustrating various phonon scattering mechanisms within a thermoelectric material, along with electronic transport of hot and cold electrons. Atomic scale defects in alloys scatter phonons due to differences in mass or due to generation of strain fields, additionally the inclusion of nanoparticles, significantly reduce lattice thermal conductivity by the additional scattering of mid- and longwavelength phonons ${ }^{[45]}$ 12

Figure 1-9: Development of bulk thermoelectric materials: the thermoelectric figure-of-merit ZT as a function of temperature and year. Green cylinders represent the p-type materials,

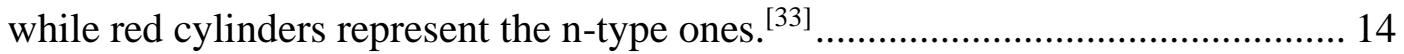

Figure 1-10: Left: Schematic comparison of various thermoelectric (TE) materials for applications of waste heat harvest and refrigeration, in terms of the temperature range of operation and the abundance and environmental friendliness of constituent elements. Rigth: The abundance of elements used in TE materials, the elements represented by darkcolored columns are $<1 \mathrm{ppm} .{ }^{[51]}$............................................ 14

Figure 1-11: Thermoelectric performance of different material families in relation with the scarcity of the constituents. Marker size is related with the dimensionless figure of merit ZT of the material. ${ }^{[55]}$ 15

Figure 1-12: Crystal structures of the layered cobalt oxides. $\mathrm{CoO}_{2}$ block in common (responsible for good electrical conductivity and key component for good Seebeck coefficient), which alternately stacks with various block layers along the $\mathrm{c}$ axis (responsible for

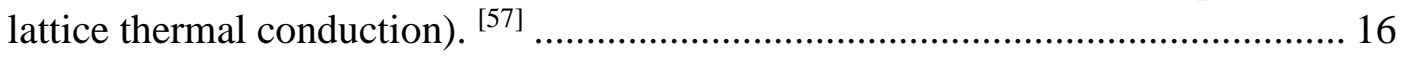


Figure 1-13: Dimensionless figure of merit $\mathrm{ZT}$ of the layered cobalt oxides comparing to conventional thermoelectric material. ${ }^{[57]}$

Figure 2-1: Representative schematic diagrams of (a) lattice misfit and (b) the resulting displacements in the two layers. Light-blue, dark-blue, and red balls are $\mathrm{Ca}, \mathrm{Co}$, and $\mathrm{O}$ atoms, respectively ${ }^{[12]}$. (c) Diagram of the crystal structure of $\mathrm{Ca}_{3} \mathrm{Co}_{4} \mathrm{O}_{9}$ perpendicular to (a) the a axis and (b) the b axis. The $\mathrm{Co} 1$ and $\mathrm{Co} 2$ sites indicated refer to $\mathrm{Co}$ atoms from the $\mathrm{Ca}_{2} \mathrm{CoO}_{3}$ layer and the $\mathrm{CoO}_{2}$ layer, respectively ${ }^{[13]}$... 24

Figure 2-2: Lamellar/multilayer nanostructures to heavily doped aligned lamellar nanostructures with embedded nano-sized inclusions. ${ }^{[23]}$ 28

Figure 2-3: Left top: XRD plots of the $\mathrm{Ca}_{3} \mathrm{CO}_{4} \mathrm{O}_{9}$ sintered specimens obtained for: (a) Solid state, (b) sol-gel. Crystallographic planes are indicated for the $\mathrm{Ca}_{3} \mathrm{Co}_{4} \mathrm{O}_{9}$ phase and $\mathrm{Ca}_{3} \mathrm{Co}_{2} \mathrm{O}_{6}$ (identified by a *).Left bottom: Temperature dependence of the power factor for $\mathrm{Ca}_{3} \mathrm{Co}_{4} \mathrm{O}_{9}$, for: $(\bullet)$ Solid state; ( $\bullet$ sol-gel. Right: SEM obtained on transversal fractured $\mathrm{Ca}_{3} \mathrm{Co}_{4} \mathrm{O}_{9}$ samples prepared by solid-state (a), and sol-gel (b) [63]

Figure 2-4: DSC/TGA curve of $\mathrm{Ca}_{3} \mathrm{Co}_{4} \mathrm{O}_{9} .^{[68]}$ 38

Figure 2-5: Comparison of the SEM images of the fractured surfaces of the $\mathrm{Ca}_{3} \mathrm{Co}_{4} \mathrm{O}_{9}$ samples prepared by classic sintering at $920{ }^{\circ} \mathrm{C}(\mathrm{CS}-920)$, hot pressing (HP), SPS sintered at $920{ }^{\circ} \mathrm{C}$ (SPT-920), and cold pressed sample sintered at $920^{\circ} \mathrm{C}$ (CP-920). The arrow indicates the stress direction. ${ }^{[64]}$ 40

Figure 4-1: procedure of fabrication polycrystalline ceramics pellets with the precursor powders made from the conventional chemical sol-gel route. 53

Figure 4-2: Principle of the Seebeck and resistivity measurement in the LSR unit. Retrieved from: linseis.com/en/products/thermoelectrics/lsr-3/............................................. 53

Figure 5-1: $\mathrm{XRD}$ powder diffraction patterns for the undoped $\mathrm{Ca}_{3} \mathrm{Co}_{4} \mathrm{O}_{9}$ and the doped $\mathrm{Ca}_{3} \mathrm{Co}_{4} \mathrm{O}_{9} \mathrm{~K}_{x}(\mathrm{x}=0.05,0.1,0.15$, and 0.2$)$ samples. 63

Figure 5-2:Cross-sectional SEM images from the fractured surface of $\mathrm{Ca}_{3} \mathrm{Co}_{4} \mathrm{O}_{9} \mathrm{~K}_{\mathrm{x}}(\mathrm{x}=0,0.05$, 0.1, 0.15, and 0.2) samples after sintering stage. (a) $\mathrm{Ca}_{3} \mathrm{Co}_{4} \mathrm{O}_{9}$, (b) $\mathrm{Ca}_{3} \mathrm{Co}_{4} \mathrm{O}_{9} \mathrm{~K}_{0.05}$,

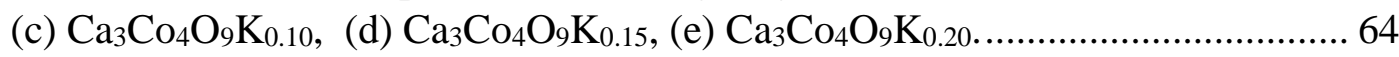

Figure 5-3: Low magnification TEM diffraction contrast image showing the EDS spot examinations performed at different location between two adjacent grains and the grain boundary for the nanostructure analysis of $\mathrm{Ca}_{3} \mathrm{Co}_{4} \mathrm{O}_{9} \mathrm{~K}_{0.1}$. Results are given in Table 3.

Figure 5-4: Temperature dependence of electrical properties for $\mathrm{Ca}_{3} \mathrm{Co}_{4} \mathrm{O}_{9} \mathrm{~K}_{\mathrm{x}}(\mathrm{x}=0,0.05,0.1$, 0.15, and 0.2). Electrical resistivity (a), Seebeck coefficient (b), and power factor (c).

Figure 5-5: Temperature dependence of (a) thermal conductivity $(\kappa-\mathrm{T})$, and (b) electronic and lattice thermal contribution for $\mathrm{Ca}_{3} \mathrm{Co}_{4} \mathrm{O}_{9} \mathrm{~K}_{\mathrm{x}}$ 69

Figure 5-6: Temperature dependence of $\mathrm{ZT}$ for $\mathrm{Ca}_{3} \mathrm{Co}_{4} \mathrm{O}_{9} \mathrm{~K}_{\mathrm{x}}$ samples. ................................... 70

Figure 6-1: Temperature dependence of electrical properties for $\mathrm{Ca}_{3} \mathrm{Co}_{4} \mathrm{O}_{9} \mathrm{Bi}_{x} \mathrm{~K}_{y}$. (a) $\rho-\mathrm{T}$, (b) $\mathrm{S}-\mathrm{T}$,

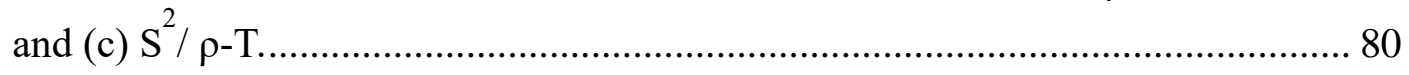


Figure 6-2: Temperature dependence of (a) thermal conductivity $(\kappa-T),(b)$ electronic and lattice thermal contribution, and (c) thermoelectric figure of merit (ZT) for $\mathrm{Ca}_{3} \mathrm{Co}_{4} \mathrm{O}_{9} \mathrm{Bi}_{\mathrm{x}} \mathrm{K}_{\mathrm{y}}$. 82

Figure 6-3: $\mathrm{XRD}$ powder diffraction patterns for the undoped $\mathrm{Ca}_{3} \mathrm{Co}_{4} \mathrm{O}_{9}$ and the doped $\mathrm{Ca}_{3} \mathrm{Co}_{4} \mathrm{O}_{9} \mathrm{Bi}_{\mathrm{x}} \mathrm{K}_{\mathrm{y}}$ samples. In all samples, monoclinic $\mathrm{Ca}_{3} \mathrm{Co}_{4} \mathrm{O}_{9}$ were observed. Nonetheless, a minor amount of $\mathrm{Co}_{3} \mathrm{O}_{4}$ (marked as *), $\mathrm{Bi}_{2} \mathrm{Ca}_{2} \mathrm{Co}_{2} \mathrm{O}_{\mathrm{x}}$ (marked as ${ }^{+}$), and an unidentified phase (marked as ${ }^{\#}$ ) were observed. 83

Figure 6-4: $\mathrm{SEM}$ images from the top surface of $\mathrm{Ca}_{3} \mathrm{Co}_{4} \mathrm{O}_{9} \mathrm{Bi}_{\mathrm{x}} \mathrm{K}_{\mathrm{y}}$ samples after sintering stage. (a) $\mathrm{Ca}_{3} \mathrm{Co}_{4} \mathrm{O}_{9}$, (b) $\mathrm{Ca}_{3} \mathrm{Co}_{4} \mathrm{O}_{9} \mathrm{~K}_{0.05}$, (c) $\mathrm{Ca}_{3} \mathrm{Co}_{4} \mathrm{O}_{9} \mathrm{~K}_{0.05} \mathrm{Bi}_{0.1}$, and (d) $\mathrm{Ca}_{3} \mathrm{Co}_{4} \mathrm{O}_{9} \mathrm{~K}_{0.05} \mathrm{Bi}_{0.15}$. 85

Figure 6-5: Cross-sectional SEM images from the fractured surface of $\mathrm{Ca}_{3} \mathrm{Co}_{4} \mathrm{O}_{9} \mathrm{Bi}_{\mathrm{x}} \mathrm{K}_{\mathrm{y}}$ samples after sintering stage. (a) $\mathrm{Ca}_{3} \mathrm{Co}_{4} \mathrm{O}_{9}$, (b) $\mathrm{Ca}_{3} \mathrm{Co}_{4} \mathrm{O}_{9} \mathrm{~K}_{0.05}$, (c) $\mathrm{Ca}_{3} \mathrm{Co}_{4} \mathrm{O}_{9} \mathrm{~K}_{0.05} \mathrm{Bi}_{0.1}$, and (d) $\mathrm{Ca}_{3} \mathrm{Co}_{4} \mathrm{O}_{9} \mathrm{~K}_{0.05} \mathrm{Bi}_{0.15}$ 86

Figure 6-6: TEM images showing the nanostructure of the $\mathrm{Ca}_{3} \mathrm{Co}_{4} \mathrm{O}_{9} \mathrm{~K}_{0.05} \mathrm{Bi}_{0.15}$ sample. The grain boundary from the crystals with different c-axis orientations. The red circles on the TEM images indicate the electron beam sample areas for the EDS data acquisition.

Figure 7-1 (a) $\rho-\mathrm{T}$, (b) $\mathrm{S}-\mathrm{T}$, and (c) $\mathrm{S}^{2} / \rho$-T show the temperature dependence of electrical properties for $\mathrm{Ca}_{3-\mathrm{x}} \mathrm{Tb}_{\mathrm{x}} \mathrm{Co}_{4} \mathrm{O}_{9}$. Inset figure shows the direction of the performed measurements. 99

Figure 7-2: Temperature dependence of: (a) $\kappa-\mathrm{T}$, (b) $\kappa_{\mathrm{e}}$ and $\kappa_{\mathrm{i}}$, and (c) $\mathrm{ZT}$ for $\mathrm{Ca}_{3-\mathrm{x}} \mathrm{Tb}_{\mathrm{x}} \mathrm{Co}_{4} \mathrm{O}_{9}$. Inset figure in (d) shows the direction of the performed measurements for $\kappa-\mathrm{T}$. 100

Figure 7-3: XRD powder diffraction patterns for $\mathrm{Ca}_{3-\mathrm{x}} \mathrm{Tb}_{\mathrm{x}} \mathrm{Co}_{4} \mathrm{O}_{9}(\mathrm{x}=0,0.05,0.30$, and 0.50) samples. In all samples, monoclinic $\mathrm{Ca}_{3} \mathrm{Co}_{4} \mathrm{O}_{9}$ was observed. Nonetheless, a minor amount of $\mathrm{Co}_{3} \mathrm{O}_{4}$ was observe as indicated by the marked peaks. (004) diffraction peak showing the shift to a higher Bragg's angle. Red line correspond to position of peak in the undoped $\mathrm{Ca}_{3} \mathrm{Co}_{4} \mathrm{O}_{9}$...... 101

Figure 7-4: SEM images of plan-view [a)-d)] and cross-section of [e)-f)] $\mathrm{Ca}_{3-\mathrm{x}} \mathrm{Tb}_{\mathrm{x}} \mathrm{Co}_{4} \mathrm{O}_{9}$ samples after sintering stage. a) and e) $\mathrm{Ca}_{3} \mathrm{Co}_{4} \mathrm{O}_{9}$, b) and f) $\mathrm{Ca}_{2.95} \mathrm{~Tb}_{0.05} \mathrm{Co}_{4} \mathrm{O}_{9}$, c) and g) $\mathrm{Ca}_{2.7} \mathrm{~Tb}_{0.3} \mathrm{Co}_{4} \mathrm{O}_{9}$, d) and h) $\mathrm{Ca}_{2.5} \mathrm{~Tb}_{0.5} \mathrm{Co}_{4} \mathrm{O}_{9}$. Arrows represent pressing direction of samples. 103

Figure 7-5: SEM images of the undoped (a) $\mathrm{Ca}_{3} \mathrm{Co}_{4} \mathrm{O}_{9}$. 104

Figure 7-6: TEM images showing the nanostructure of the $\mathrm{Ca}_{2.5} \mathrm{~Tb}_{0.5} \mathrm{Co}_{4} \mathrm{O}_{9}$ sample. (a) The grain boundary from the crystals with different c-axis orientations. The red circles on the TEM images indicate the electron beam sample areas for the EDS data acquisition. (b) TEM showing that adjacent crystals have a large amount of high angle GBs between them 105

Figure 7-7: $\mathrm{Co} 2 \mathrm{p}_{3 / 2}$ and $2 \mathrm{p}_{1 / 2}$ peaks from XPS spectrum of $\mathrm{Ca}_{3-\mathrm{x}} \mathrm{Tb}_{\mathrm{x}} \mathrm{Co}_{4} \mathrm{O}_{9}$ samples. Inset figure showing Co $2 \mathrm{p}_{3 / 2}$ peaks shifting to a lower binding energy. 106

Figure 7-8: Deconvolution of $\mathrm{Co} 2 \mathrm{p}_{3 / 2}$ peak from XPS spectrums of $\mathrm{Ca}_{3-\mathrm{x}} \mathrm{Tb}_{\mathrm{x}} \mathrm{Co}_{4} \mathrm{O}_{9}$ samples. 107 Figure 8-1: Temperature dependence of electrical properties for $\mathrm{Ca}_{3-\mathrm{x}} \mathrm{Tb}_{\mathrm{x}} \mathrm{Co}_{4} \mathrm{O}_{9} \mathrm{~K}_{\mathrm{y}}(\mathrm{x}=0, \mathrm{y}=0$; $\mathrm{x}=0.05, \mathrm{y}=0 ; \mathrm{x}=0.5, \mathrm{y}=0 ; \mathrm{x}=0.05, \mathrm{y}=0.1 ; \mathrm{x}=0.5, \mathrm{y}=0.1$ ). (a) $\rho-\mathrm{T}$, (b) $\mathrm{S}-\mathrm{T}$, and (c) $\mathrm{S}^{2}$ / $\rho-\mathrm{T}$. 119 
Figure 8-2: Temperature dependence of (a) thermal conductivity $(\kappa-T)$, and (b) thermoelectric figure of merit $(\mathrm{ZT})$ for $\mathrm{Ca}_{3-\mathrm{x}} \mathrm{Tb}_{\mathrm{x}} \mathrm{Co}_{4} \mathrm{O}_{9} \mathrm{~K}_{\mathrm{y}}(\mathrm{x}=0, \mathrm{y}=0 ; \mathrm{x}=0.05, \mathrm{y}=0 ; \mathrm{x}=0.5, \mathrm{y}=0$; $\mathrm{x}=0.05, \mathrm{y}=0.1 ; \mathrm{x}=0.5, \mathrm{y}=0.1)$.

Figure 8-3: XRD powder diffraction patterns for the undoped $\mathrm{Ca}_{3} \mathrm{Co}_{4} \mathrm{O}_{9}$ and the doped $\mathrm{Ca}_{3 \text { - }}$ ${ }_{\mathrm{x}} \mathrm{Tb}_{\mathrm{x}} \mathrm{Co}_{4} \mathrm{O}_{9} \mathrm{~K}_{\mathrm{y}}(\mathrm{x}=0.05, \mathrm{y}=0 ; \mathrm{x}=0.5, \mathrm{y}=0 ; \mathrm{x}=0.05, \mathrm{y}=0.1 ; \mathrm{x}=0.5, \mathrm{y}=0.1)$ samples. In all samples, monoclinic $\mathrm{Ca}_{3} \mathrm{Co}_{4} \mathrm{O}_{9}$ were observed (ICDD cards \#4-16-860). Nonetheless, a minor amount of $\mathrm{Co}_{3} \mathrm{O}_{4}$ was observed as indicated by the marked peaks (ICDD cards \# 4-8-3173).

Figure 8-4: $\mathrm{SEM}$ images from the top surface of $\mathrm{Ca}_{3-\mathrm{x}} \mathrm{Tb}_{\mathrm{x}} \mathrm{Co}_{4} \mathrm{O}_{9} \mathrm{~K}_{\mathrm{y}}(\mathrm{x}=0, \mathrm{y}=0 ; \mathrm{x}=0.05, \mathrm{y}=0$; $\mathrm{x}=0.5, \mathrm{y}=0 ; \mathrm{x}=0.05, \mathrm{y}=0.1 ; \mathrm{x}=0.5, \mathrm{y}=0.1$ ) samples after sintering stage. (a) $\mathrm{Ca}_{3} \mathrm{Co}_{4} \mathrm{O}_{9}$, (b) $\mathrm{Ca}_{2.95} \mathrm{~Tb}_{0.05} \mathrm{Co}_{4} \mathrm{O}_{9}$, (c) $\mathrm{Ca}_{2.5} \mathrm{~Tb}_{0.5} \mathrm{Co}_{4} \mathrm{O}_{9}$, (d) $\mathrm{Ca}_{2.95} \mathrm{~Tb}_{0.05} \mathrm{Co}_{4} \mathrm{O}_{9} \mathrm{~K}_{0.1}$, (e)

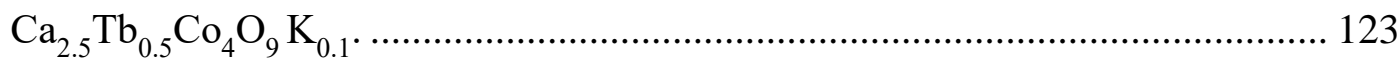

Figure 8-5: Cross-sectional SEM images from the fractured surface of $\mathrm{Ca}_{3-\mathrm{x}} \mathrm{Tb}_{\mathrm{x}} \mathrm{Co}_{4} \mathrm{O}_{9} \mathrm{~K}_{\mathrm{y}}(\mathrm{x}=0$, $\mathrm{y}=0 ; \mathrm{x}=0.05, \mathrm{y}=0 ; \mathrm{x}=0.5, \mathrm{y}=0 ; \mathrm{x}=0.05, \mathrm{y}=0.1 ; \mathrm{x}=0.5, \mathrm{y}=0.1)$ samples after sintering stage. (a) $\mathrm{Ca}_{3} \mathrm{Co}_{4} \mathrm{O}_{9}$, (b) $\mathrm{Ca}_{2.95} \mathrm{~Tb}_{0.05} \mathrm{Co}_{4} \mathrm{O}_{9}$, (c) $\mathrm{Ca}_{2.5} \mathrm{~Tb}_{0.5} \mathrm{Co}_{4} \mathrm{O}_{9}$, (d) $\mathrm{Ca}_{2.95} \mathrm{~Tb}_{0.05} \mathrm{Co}_{4} \mathrm{O}_{9} \mathrm{~K}_{0.1}, \quad$ (e) $\mathrm{Ca}_{2.5} \mathrm{~Tb}_{0.5} \mathrm{Co}_{4} \mathrm{O}_{9} \mathrm{~K}_{0.1}$ 124

Figure 8-6: TEM images showing the nanostructure of the $\mathrm{Ca}_{2.95} \mathrm{~Tb}_{0.05} \mathrm{Co}_{4} \mathrm{O}_{9} \mathrm{~K}_{0.1}$ sample. (a) The grain boundary from the crystals with different c-axis orientations. The red circles on the TEM images indicate the electron beam sample areas for the EDS data acquisition 125

Figure 9-1: Timeline of the improvement in the $\mathrm{ZT}$ of $\mathrm{Ca}_{3} \mathrm{Co}_{4} \mathrm{O}_{9}$ polycrystalline materials by the addition of different doping elements. ${ }^{(18,19,21,22, ~ 25-27, ~ 29-31, ~ 34, ~ 35, ~ 38, ~ 40, ~ 42, ~ 44, ~ 45, ~ 48, ~ 49, ~ 53, ~}$ $54)$ *ZT value of this work is also reported. The goal of reaching a ZT material over 1 for commercial applications would be achievable within a few years. 136

Figure 9-2: (a) $\rho$-T, (b) $S$-T, and (c) $S^{2} / \rho$-T show the temperature dependence of properties for $\mathrm{Ca}_{3} \mathrm{Co}_{4} \mathrm{O}_{9}$ with dual dopants. Inset figure(a) shows the direction of the performed measurements. 141

Figure 9-3: Temperature dependence of: (a) total thermal conductivity $(\kappa-T)$, (b) electronic contribution $\left(\kappa_{\mathrm{e}}\right)$ and lattice contribution $\left(\kappa_{\mathrm{i}}\right)$, and $(\mathrm{c}) \mathrm{ZT}$ for $\mathrm{Ca}_{3} \mathrm{Co}_{4} \mathrm{O}_{9}$ with dual dopants. Inset figure in (a) shows the direction of the performed measurements for $\kappa-\mathrm{T}$. 144

Figure 9-4: XRD powder diffraction patterns for the undoped and the doped $\mathrm{Ca}_{3} \mathrm{Co}_{4} \mathrm{O}_{9}$ samples. In all samples, monoclinic $\mathrm{Ca}_{3} \mathrm{CO}_{4} \mathrm{O}_{9}$ was observed. Nonetheless, a minor amount of $\mathrm{Co}_{3} \mathrm{O}_{4}$ and $\mathrm{Ca}_{3} \mathrm{Co}_{2} \mathrm{O}_{6}$ was observed as indicated by the marked peaks * and ${ }^{+}$, respectively. (003) diffraction peak showing the shift to a lower Bragg's angle. Red line correspond to position of peak in the undoped $\mathrm{Ca}_{3} \mathrm{Co}_{4} \mathrm{O}_{9}$..... 145

Figure 9-5: SEM plan-view images from the surface of $\mathrm{Ca}_{3} \mathrm{Co}_{4} \mathrm{O}_{9}$ samples with dual dopants after sintering stage. 147

Figure 9-6: SEM cross-sectional images from the fractured surface of $\mathrm{Ca}_{3} \mathrm{Co}_{4} \mathrm{O}_{9}$ samples with dual dopants after sintering stage. Arrows represent the pressing direction of the samples. 148 
Figure 9-7: TEM images showing the nanostructure of the doped $\mathrm{Ca}_{3} \mathrm{Co}_{4} \mathrm{O}_{9}$ sample. (a) The grain boundary from the crystals with different c-axis orientations. The red circles on the TEM images indicate the electron beam sample areas for the EDS data acquisition. (b) TEM showing adjacent crystals having a preferred crystal orientation in the nanolamellas. (c)-(d) HRTEM showing the low angle GBs between neighboring crystals. 151

Figure 9-8: $\mathrm{Co} 2 \mathrm{p}_{3 / 2}$ and $2 \mathrm{p}_{1 / 2}$ peaks from XPS spectrum of $\mathrm{Ca}_{3} \mathrm{Co}_{4} \mathrm{O}_{9}$ samples with dual dopants. Inset figure showing $\mathrm{Co} 2 \mathrm{p}_{3 / 2}$ peaks shifting to a lower binding energy. 153

Figure 9-9: Deconvolution of $\mathrm{Co} 2 \mathrm{p}_{3 / 2}$ peak from XPS of $\mathrm{Ca}_{3} \mathrm{Co}_{4} \mathrm{O}_{9}$ samples with dual dopants. 154

Figure 9-10: Temperature dependence of thermoelectric properties for different state-of-the-art ptype materials: (a) $\mathrm{S}^{2} / \rho-\mathrm{T}$, (b) $\kappa-\mathrm{T}$, and (c) ZT. 159

Figure 9-11: Temperature dependence of thermoelectric properties for different dopant species and doping level of high-performance $\mathrm{Ca}_{3} \mathrm{Co}_{4} \mathrm{O}_{9}$ ceramics. 162

Figure 10-1: Schematic showing the systematic microstructure evolution of $\mathrm{Ca}_{3} \mathrm{Co}_{4} \mathrm{O}_{9} \ldots \ldots \ldots .171$

Figure 10-2: Multiple leg setup for prototype of $\mathrm{Ca}_{3} \mathrm{Co}_{4} \mathrm{O}_{9}$ legs as p-type material and $\mathrm{CaMnO}_{3}$ as n-type material using silver paste for bonding the legs with the silver foil and alumina. 172

Figure 10-3: Characteristic of TE modules as function of the electrical current. The electrical power densities were calculated from the active area of the unicouples 173 


\section{List of Tables}

Table 1-1: U.S. Manufacturing Industries Ranked by Process Heating Energy Use in 2010. ${ }^{[4]} \ldots 3$

Table 5-1: Apparent density of the $\mathrm{Ca}_{3} \mathrm{Co}_{4} \mathrm{O}_{9} \mathrm{~K}_{x}$ samples.

Table 5-2: Lattice parameters $a, b_{1}, b_{2}$, $c$, and $\beta$ for $\mathrm{Ca}_{3} \mathrm{Co}_{4} \mathrm{O}_{9} \mathrm{~K}_{\mathrm{x}}(\mathrm{x}=0,0.05,0.1,0.15$, and 0.2) samples. $b_{1}$ and $b_{2}$ refer to $b$-axis lattice parameters in the rock-salt layer $\mathrm{Ca}_{2} \mathrm{CoO}_{3}$ and the $\mathrm{CoO}_{2}$ layer, respectively.

Table 5-3: EDS spot examination for $\mathrm{Ca}_{3} \mathrm{Co}_{4} \mathrm{O}_{9} \mathrm{~K}_{0.1}$ sample showing the chemistry of the interior of the grain (spots 1, 3, 4, 6, 7, 9, 10, 12, 13, and 15) and between grain boundary areas (spots $2,5,8,11,14$ ), respectively. The symbol (-) indicates the absence of the

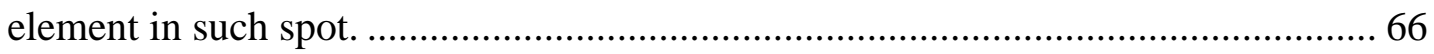

Table 6-1: Apparent density of the $\mathrm{Ca}_{3} \mathrm{Co}_{4} \mathrm{O}_{9} \mathrm{Bi}_{\mathrm{x}} \mathrm{K}$ samples............................................... 78

Table 6-2: Lattice parameters $a, b_{1}, b_{2}$, c, and $\beta$ for the $\mathrm{Ca}_{3} \mathrm{Co}_{4} \mathrm{O}_{9} \mathrm{Bi}_{\mathrm{x}} \mathrm{K}_{\mathrm{y}}$ samples. $\mathrm{b}_{1}$ and $\mathrm{b}_{2}$ refer to b-axis lattice parameters in the rock-salt layer $\mathrm{Ca}_{2} \mathrm{CoO}_{3}$ and the $\mathrm{CoO}_{2}$ layer,

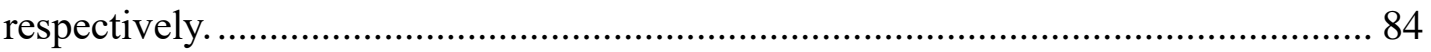

Table 6-3: Chemistry of the grain interior and grain boundaries of $\mathrm{Ca}_{3} \mathrm{Co}_{4} \mathrm{O}_{9} \mathrm{Bi}_{0.15} \mathrm{~K}_{0.05}$ for different sites labeled in the TEM image. ..................................................... 87

Table 7-1: Apparent density of the $\mathrm{Ca}_{3-\mathrm{x}} \mathrm{Tb}_{\mathrm{x}} \mathrm{Co}_{4} \mathrm{O}_{9}$ samples. ................................................. 97

Table 7-2: Lattice parameters $a, b_{1}, b_{2}, c$, and $\beta$ for $\mathrm{Ca}_{3-\mathrm{x}} \mathrm{Tb}_{\mathrm{x}} \mathrm{Co}_{4} \mathrm{O}_{9}$ samples. $\mathrm{b}_{1}$ and $\mathrm{b}_{2}$ refer to $b$ axis lattice parameters in the rock-salt layer $\mathrm{Ca}_{2} \mathrm{CoO}_{3}$ and the $\mathrm{CoO}_{2}$ layer, respectively. 102

Table 7-3: Chemistry of the grain interior and grain boundaries of $\mathrm{Ca}_{2.5} \mathrm{~Tb}_{0.5} \mathrm{Co}_{4} \mathrm{O}_{9}$ for different sites labeled in the TEM image in Figure 7-6(a)................................................. 105

Table 7-4: Ratios of $\mathrm{Co}^{4+}, \mathrm{Co}^{3+}$ and $\mathrm{Co}^{2+}$ from the XPS deconvolution of the Co 2p $\mathrm{p}_{3 / 2}$ peak... 107

Table 8-1: Apparent density of the $\mathrm{Ca}_{3-\mathrm{x}} \mathrm{Tb}_{\mathrm{x}} \mathrm{Co}_{4} \mathrm{O}_{9} \mathrm{~K}_{\mathrm{y}}$ samples. ......................................... 117

Table 8-2: Lattice parameters $a, b_{1}, b_{2}, c$, and $\beta$ for $\mathrm{Ca}_{3-\mathrm{x}} \mathrm{Tb}_{\mathrm{x}} \mathrm{Co}_{4} \mathrm{O}_{9} \mathrm{~K}_{\mathrm{y}}(\mathrm{x}=0, \mathrm{y}=0 ; \mathrm{x}=0.05, \mathrm{y}=0$; $\mathrm{x}=0.5, \mathrm{y}=0 ; \mathrm{x}=0.05, \mathrm{y}=0.1 ; \mathrm{x}=0.5, \mathrm{y}=0.1)$ samples. $\mathrm{b}_{1}$ and $\mathrm{b}_{2}$ refer to $\mathrm{b}$-axis lattice parameters in the rock-salt layer $\mathrm{Ca}_{2} \mathrm{CoO}_{3}$ and the $\mathrm{CoO}_{2}$ layer, respectively......... 122

Table 8-3: Chemistry of the grain interior and grain boundaries of $\mathrm{Ca}_{2.95} \mathrm{~Tb}_{0.05} \mathrm{Co}_{4} \mathrm{O}_{9} \mathrm{~K}_{0.1}$ for different sites labeled in the TEM image in Figure 8-6....................................... 126

Table 9-1: Progression of the performance of $\mathrm{Ca}_{3} \mathrm{Co}_{4} \mathrm{O}_{9}$ thermoelectric material. .................. 135

Table 9-2: Apparent density of the $\mathrm{Ca}_{3} \mathrm{Co}_{4} \mathrm{O}_{9}$ samples..................................................... 139

Table 9-3: Lattice parameters $a, b_{1}, b_{2}, c$, and $\beta$ for the single doped and dual doped $\mathrm{Ca}_{3} \mathrm{Co}_{4} \mathrm{O}_{9}$ samples. $b_{1}$ and $b_{2}$ refer to $b$-axis lattice parameters in rock-salt layer $\mathrm{Ca}_{2} \mathrm{CoO}_{3}$ and

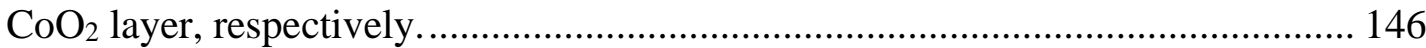

Table 9-4: Lotgering factor for the dual doped $\mathrm{Ca}_{3} \mathrm{Co}_{4} \mathrm{O}_{9}$ samples ..................................... 150

Table 9-5: Chemistry of the grain interior and grain boundaries of the dual doped $\mathrm{Ca}_{3} \mathrm{Co}_{4} \mathrm{O}_{9}$ sample for different sites labeled in the TEM image in Figure 9-7(a). ................. 152

Table 9-6: Ratios of $\mathrm{Co}^{4+}, \mathrm{Co}^{3+}$ and $\mathrm{Co}^{2+}$ from the XPS deconvolution of the Co $2 \mathrm{p}_{3 / 2}$ peak... 154

Table 10-1: Summary of peak improvements for different analyzed materials by

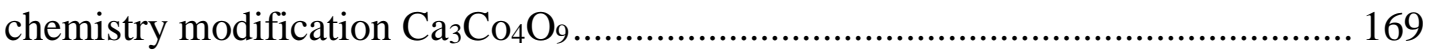




\section{List of Nomenclature}

\begin{tabular}{|c|c|c|}
\hline$C_{v}$ & Specific heat a constant volume & {$[\mathrm{J} / \mathrm{K}]$} \\
\hline$C_{p}$ & Specific heat a constant pressure & {$[\mathrm{J} / \mathrm{K}]$} \\
\hline$e$ & Electron charge & {$[\mathrm{C}]$} \\
\hline$h$ & Planck constant & {$[\mathrm{J} \mathrm{s}]$} \\
\hline$k_{b}$ & Boltzmann constant & {$[\mathrm{J} / \mathrm{K}]$} \\
\hline$l$ & Mean free path of the phonons & {$[\mathrm{m}]$} \\
\hline$L$ & Lorenz number & {$\left[\mathrm{W} \Omega \mathrm{K}^{-2}\right]$} \\
\hline$m$ & Density of states effective mass of carriers & {$[\mathrm{kg}]$} \\
\hline$n$ & Charge carrier density & {$\left[\mathrm{m}^{-3}\right]$} \\
\hline$S^{2} \sigma$ & Electrical power factor $(\mathrm{PF})$ & {$\left[\mathrm{W} / \mathrm{mK}^{2}\right]$} \\
\hline$S$ & Seebeck coefficient & {$[\mathrm{V} / \mathrm{K}]$} \\
\hline$T$ & Absolute temperature & {$[\mathrm{K}]$} \\
\hline$T_{h}$ & Hot side temperature & {$[\mathrm{K}]$} \\
\hline$T_{c}$ & Cold side temperature & {$[\mathrm{K}]$} \\
\hline$v_{s}$ & Velocity of the sound & {$[\mathrm{m} / \mathrm{s}]$} \\
\hline$Z$ & Figure of merit & {$\left[\mathrm{K}^{-1}\right]$} \\
\hline$Z T$ & Dimensionless figure of merit & [ ] \\
\hline$\Delta T$ & Temperature difference & {$[\mathrm{K}]$} \\
\hline$\eta$ & Maximum efficiency of thermoelectric device & {$[\%]$} \\
\hline$\theta$ & Bragg's angle & {$\left[{ }^{\circ}\right]$} \\
\hline$\kappa_{e l}$ & Electronic contribution of thermal conductivity & {$[\mathrm{W} / \mathrm{m} \mathrm{K}]$} \\
\hline$\kappa_{p h}$ & Lattice contribution of thermal conductivity & {$[\mathrm{W} / \mathrm{m} \mathrm{K}]$} \\
\hline$\kappa_{\text {tot }}$ & Total thermal conductivity & {$[\mathrm{W} / \mathrm{m} \mathrm{K}]$} \\
\hline$\mu$ & Carrier mobility & {$\left[\mathrm{m}^{2} / \mathrm{Vs}\right]$} \\
\hline$\rho$ & Electrical resistivity & {$[1 / \sigma]$} \\
\hline$\sigma$ & Electrical conductivity & {$[\mathrm{S} / \mathrm{m}]$} \\
\hline
\end{tabular}




\section{Chapter 1. Introduction to Thermoelectric Materials}

\section{1 Energy consumption}

Today's globalized world fiercely demands an unprecedented amount of energy. Humankind needs to evolve as the countless emerging new technologies grow, fulfillment of such world energy consumption requires an increasing necessity to ensure an adequate supply of energy in the future. Nonetheless, in order to fulfil those needs companies fight to improve their cost competitiveness, and at the same time provide an outstanding level of environmental performance in their processes, owing to the fact that the growing demand for energy requires a source of energy for the future that not only focuses on fossil fuel sources and conventional technologies but on a broader range of environmental friendly energies with cutting-edge renewable energy technology. Additionally, consumption of renewable energy and efficient use of fuel will reduce ecological footprint of society which is vastly influenced by the use of fossil fuels. According to the International Energy Agency the total primary energy supply has increased by almost 2.5 times between 1971 and $2015^{[1]}$, relying mostly in fossil fuels as the predominant fuel source to produce energy, as shown in Figure 1-1 left. Remarkably, in the long term world energy consumption for 2040 is projected to be 56 percent higher in comparison to $2010^{[2]}$ (depicted in Figure 1-1, Right). Therefore,

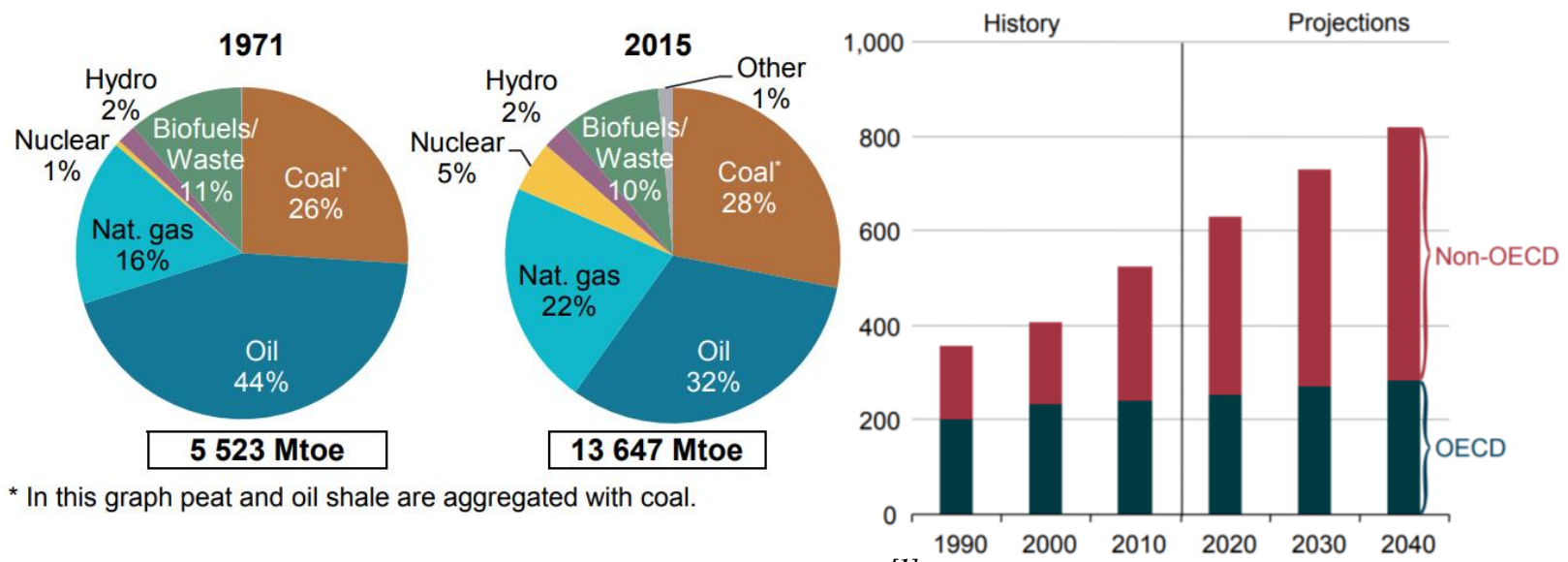

Figure 1-1: Left: Total primary energy supply by fuel. ${ }^{[1]}$ Mtoe: Million Tons of Oil Equivalent. Right: World energy consumption projections, 1990-2040 (quadrillion Btu). ${ }^{[2]}$ 
innovative solutions must be investigated in worldwide efforts to make renewable energy more efficient, for this reason researchers have been working to develop alternative materials and devices to improve the energy consumption efficiency.

\section{2 Waste heat potential}

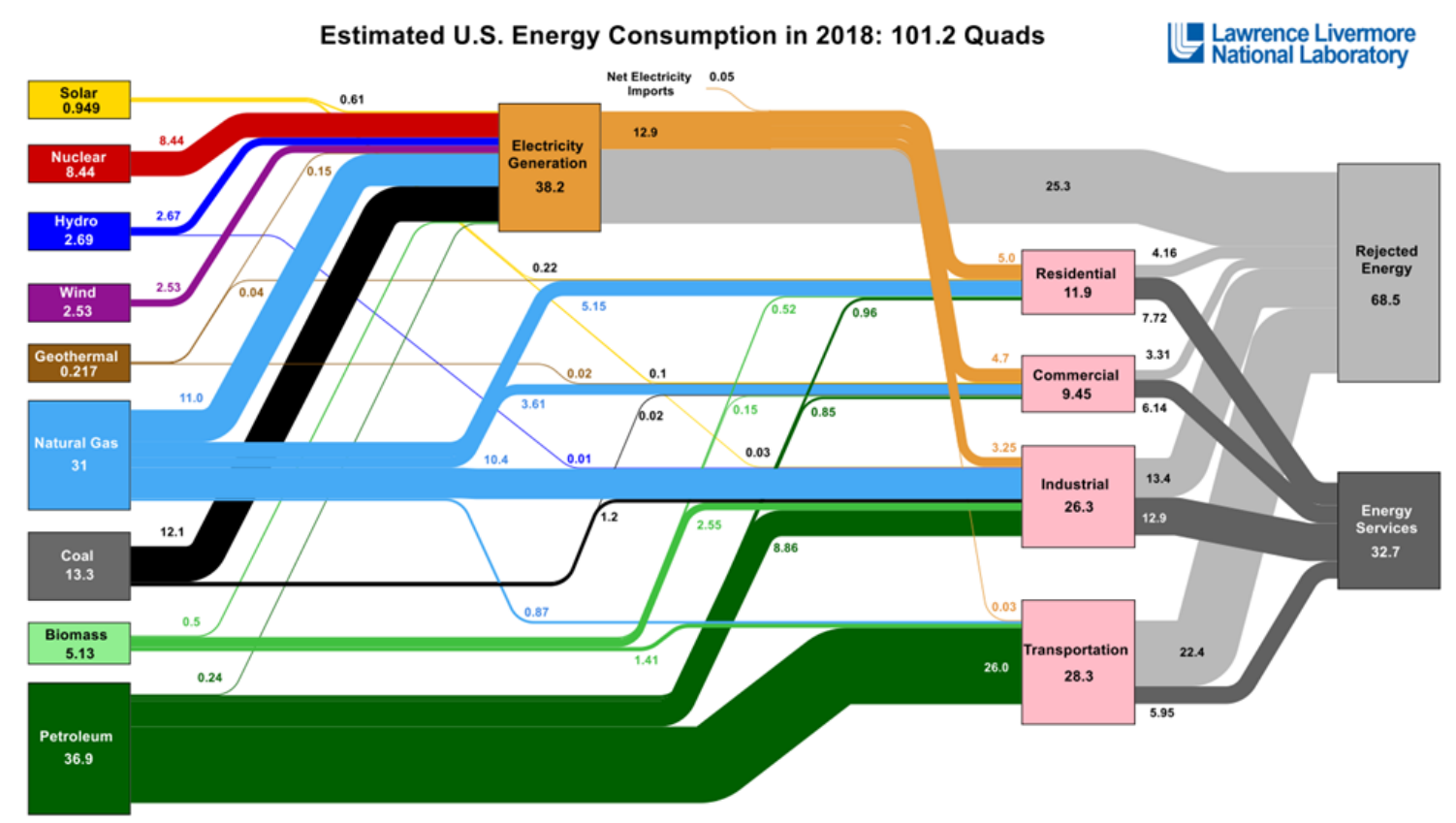

Figure 1-2: Estimated U.S. Energy Consumption in 2018 $8^{[3]}$

According to the estimated U.S. energy consumption in $2018^{[3]}$, about $68 \%$ of all energy is lost after conversion as rejected energy. Focusing specifically on the industrial manufacturing sector, the percentage of process heating energy lost accounts for $36 \%$ of the total U.S. industry energy use $^{[4]}$. Iron and steel industry lost $46 \%$ of the of process heating energy, as shown in Table 1-1. Therefore, waste heat recovery represents an extraordinary opportunity to utilize the thermal losses and provide additional energy to supply the demand in industrial processes. Additionally, given the high economic impact to take advantage of the calorific energy wasted in the industrial processes to save energy in order to minimize cost of production, an opportunity analysis for recovering energy from industrial waste heat and emissions from report from Pacific Northwest National Laboratory (PNNL) focused on the potential opportunities to recovering chemical and 
thermal emissions from U.S. industry, it is reported in this analysis that capturing and redeploying this energy could generate an economic benefit of approximately $\$ 5$ Billion dollars if realized ${ }^{[5]}$, the estimated energy recovery opportunity represents $4.3 \%$ of the total energy used by U. S. industry. ${ }^{[4]}$

Table 1-1: U.S. Manufacturing Industries Ranked by Process Heating Energy Use in 2010. ${ }^{[4]}$

\begin{tabular}{lcccc}
\hline Manufacturing Industry & $\begin{array}{l}\text { Process } \\
\text { heating } \\
\text { energy use } \\
\text { (TBtu) }\end{array}$ & $\begin{array}{l}\text { Process } \\
\text { heating energy } \\
\text { losses (TBtu) }\end{array}$ & $\begin{array}{l}\text { Percent of process } \\
\text { heating energy lost } \\
\text { within sector (\%) }\end{array}$ & $\begin{array}{l}\text { Percent of total U.S. } \\
\text { manufacturing } \\
\text { process heating } \\
\text { energy losses (\%) }\end{array}$ \\
\hline Petroleum refining & 2,250 & 397 & $18 \%$ & $15 \%$ \\
\hline Chemicals & 1,455 & 328 & $23 \%$ & $13 \%$ \\
\hline Forest products & 980 & 701 & $72 \%$ & $27 \%$ \\
\hline Iron and steel & 729 & 334 & $46 \%$ & $13 \%$ \\
\hline Food and beverage & 519 & 293 & $56 \%$ & $11 \%$ \\
\hline Cement & 213 & 84 & $39 \%$ & $3 \%$ \\
\hline Glass & 161 & 88 & $55 \%$ & $1 \%$ \\
\hline Fabricated metals & 138 & 49 & $36 \%$ & $1 \%$ \\
\hline Rubber products & 87 & 20 & $23 \%$ & $1 \%$ \\
\hline Alumina and aluminum & 82 & 37 & $45 \%$ & $1 \%$ \\
\hline Transportation equipment & 65 & 23 & $35 \%$ & $1 \%$ \\
\hline Foundries & 61 & 28 & $46 \%$ & $5 \%$ \\
\hline Electronics equipment & 43 & 15 & $35 \%$ & $100 \%$ \\
\hline Textiles & 41 & 23 & $56 \%$ & \\
\hline Machinery & 38 & 13 & $34 \%$ & $39 \%$ \\
\hline Other Manufacturing & 342 & 134 & $36 \%$ & \\
\hline All Manufacturing & 7,204 & 2,567 & & \\
\hline
\end{tabular}

Although no particular waste heat recovery technology is capable of harness all the diverse forms of thermal loss opportunities (from low to high temperature waste heat), several options represent increasing potential for integration in industrial processes ${ }^{[6]}$. In this assessment, thermoelectric waste-energy recovery systems are among the most promising conversion technologies. Thermoelectric materials provide a resource to develop solid-state energy conversion devices capable of directly convert heat to electricity with the appeal in terms of simplicity. In this 
assessment, remote industrial facilities with abundant waste heat and expensive energy resources might benefit from waste heat recovery. ${ }^{[7]}$

\section{3 Historical background of thermoelectricity}

The ability to use heat as a source to produce electricity is not a novel phenomenon, in fact it dates back to the $19^{\text {th }}$ century. In 1821 the German physicist Thomas Seebeck observed that if a circuit made from two dissimilar materials is subject to a temperature difference, one could obtain a small current flow from the hot side to the cold side ${ }^{[8]}$ (Figure 1-3 ${ }^{[9]}$ ), such effect is known as the Seebeck effect in honor to the German physicist.

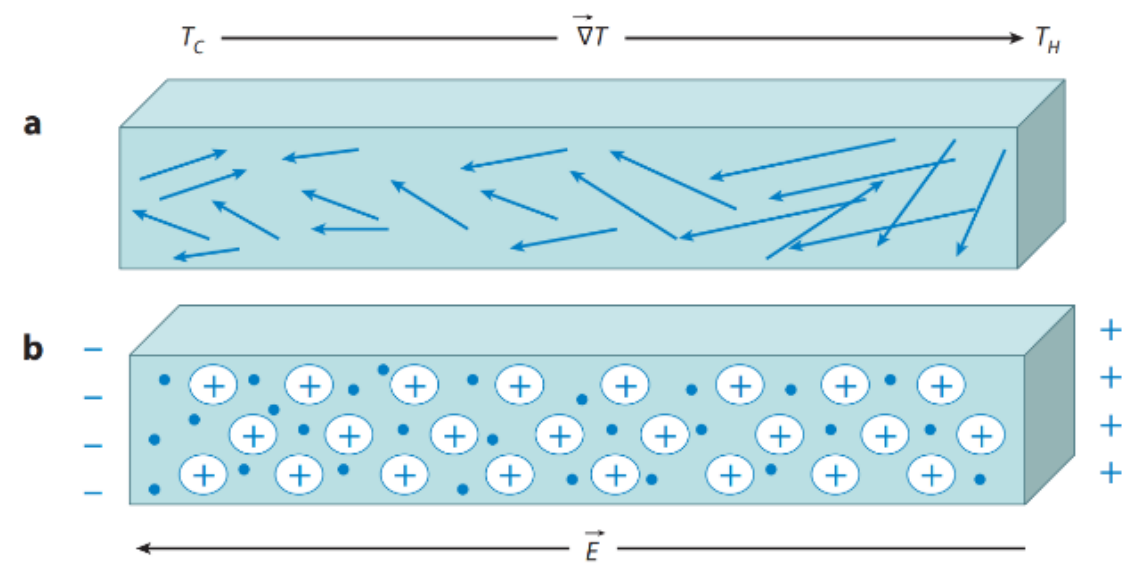

Figure 1-3: A depiction of the thermoelectric effect when a material is subjected to an external temperature gradient and the system is in equilibrium. (a) The more energetic electrons have a longer mean free path. (b) These more energetic electrons (denoted by blue dots) migrate to a lower potential until an electric field $(E)$ is developed to oppose any further flow of electrons. ${ }^{[9]}$

In the same way, French physicist Jean Charles Peltier found it was possible to use an electrical current to produce a temperature difference at the connection of two different metals ${ }^{[10]}$, later it was discovered that the direction of the current can be changed in order to produce a hot end or a cold end ${ }^{[11]}$, the heat generated or absorbed at the material is proportional to the electrical current supplied and is known as the Peltier coefficient. In 1851 William Thomson found that as a matter of fact both the Seebeck and the Peltier effect are related ${ }^{[12]}$, such a thermoelectric material could be used to either generate a power current when subject to a temperature difference or pump heat 
if supplied with an electric current. Thomson stated that the heat effects which cause the current take place, not only at the junctions, but along the materials themselves ${ }^{[13]}$. After numerous demonstrations and studies about thermoelectric materials, in 1911 Altenkirch $^{[14]}$ correctly derived $^{2}$ the maximum efficiency of a thermoelectric power generator and performance of a Peltier cooler. The efforts of Altenkirch lead to the basis of the concept of the thermoelectric figure of merit (ZT). The findings serve to identify the desirable characteristic of a good thermoelectric material, such material must have: low thermal conductivity necessary to maintain a large temperature gradient between the hot and cold sides in a thermoelectric system, low electrical resistivity, otherwise electron scattering generates heat on both sides of the barrier and throughout the materials due to the Joule effect, and a high Seebeck coefficient ${ }^{[15]}$.
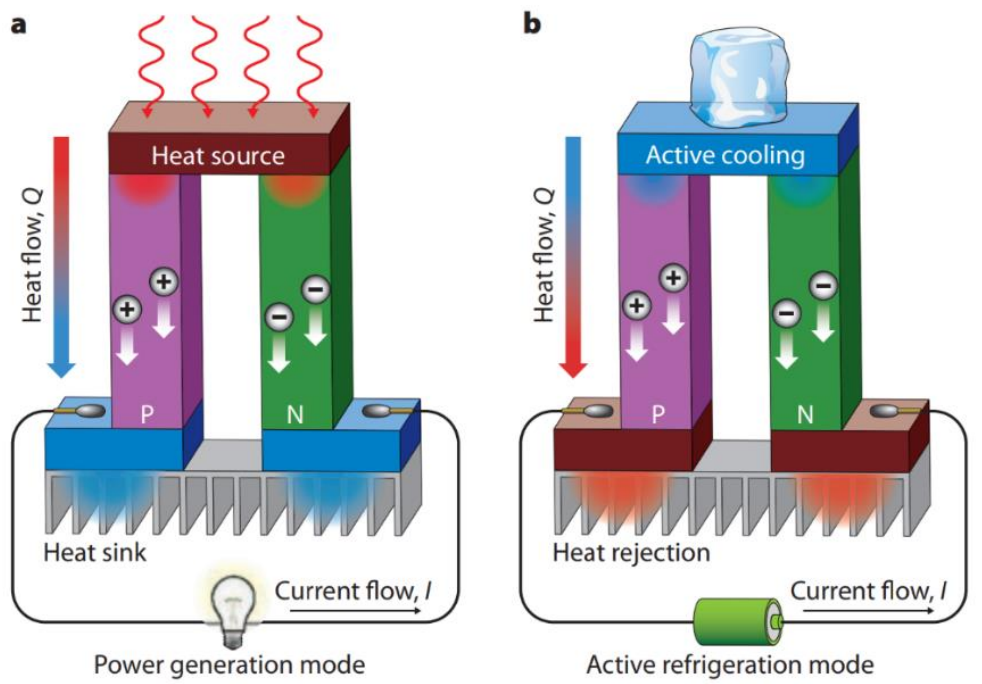

Figure 1-4: Schematic illustrations of a thermoelectric module for (a) power generation (Seebeck effect) and (b) active refrigeration (Peltier effect). ${ }^{[16]}$

Since the discovery of the effects on thermoelectric materials, numerous attempts were made to implement thermoelectric for the use in the industrial sector with only small success ${ }^{[17]}$. Nonetheles, during 1950s the arrival of doped semiconductor materials with small band gap, which were discovered to have considerable higher thermoelectric performances than pure metals, revitalized the attention in thermoelectrics ${ }^{[18]}$. In 1961, research conducted at the National 
Aeronautics and Space Administration (NASA) established the radioisotope thermoelectric generators to provide electrical power for their U. S. space program ${ }^{[19]}$. They conceived that the simplicity and reliability of thermoelectrics was to be the most viable way to generate electric power remotely, since then it has been an important part of the U. S. space program ${ }^{[20]}$. However, thermoelectric generators used in space applications are out of reach for potential commercial applications due to the excessive cost of materials manufacture. Additionally, most of the materials do not meet targets necessary to be commercial worthwhile. Therefore, finding new materials with superior properties to maximize power-generation efficiency is crucial to develop thermoelectric devices for future commercial applications. Is for that reason, thermoelectric materials represent an enthusiastic research topic for current material scientist.
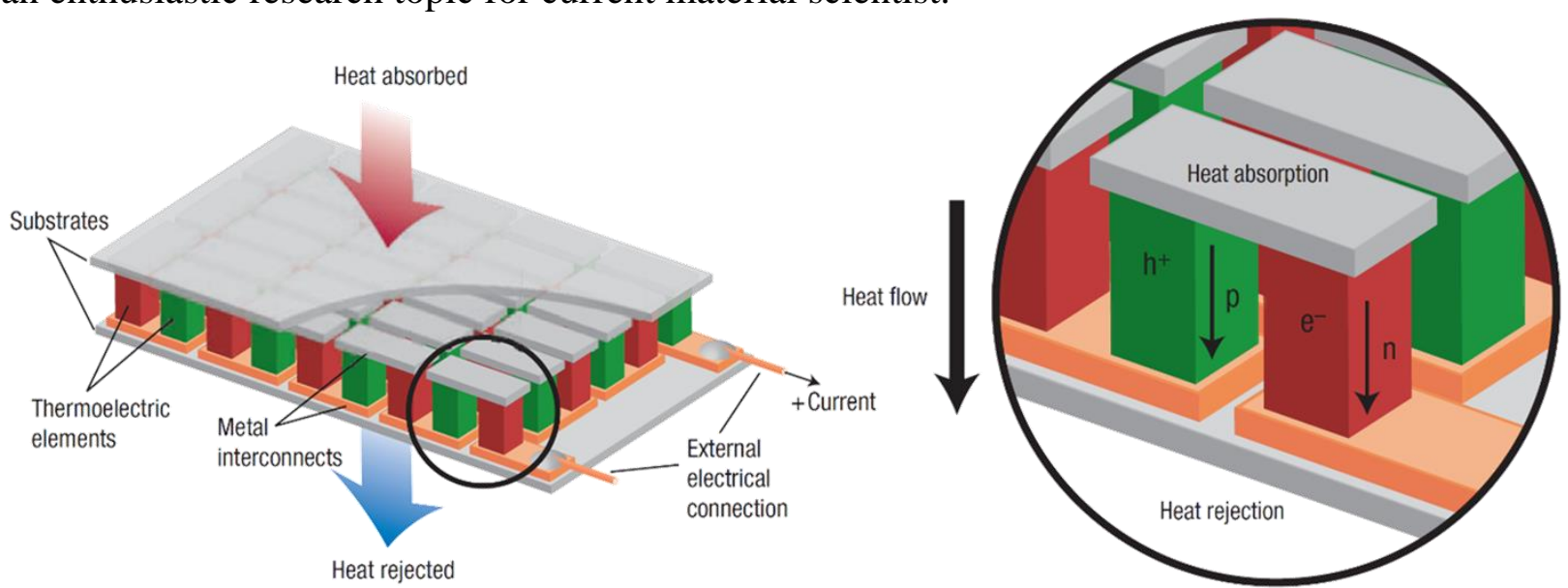

Figure 1-5: Thermoelectric module showing the direction of charge flow on power generation. ${ }^{[15]}$

\section{4 Thermoelectric properties}

Thermoelectric power generator devices are made up of multiple p-type (material containing free holes, positively charged) and n-type (materials containing free electrons, negatively charged) legs which are connected electrically in series and thermally in parallel. The p-type and n-type legs are joined typically by a metallic interconnection (must be an excellent electrical conductor) and the device is placed with the top side connected to a heat source and the bottom to a heat $\sin ^{[21]}$, 
as shown in Figure 1-5. Several advantages provide the thermoelectric generators such as: a long lifespan, no moving parts, hence no maintenance, no scale effect, and noiseless operations ${ }^{[22]}$.

The performance of materials used for thermoelectric power devices is evaluated by the equation of the figure of merit $\mathrm{Z}\left[\mathrm{K}^{-1}\right]$ :

$$
Z=\frac{S^{2} \sigma}{\kappa_{t o t}}=\frac{S^{2} \sigma}{\kappa_{e l}+\kappa_{p h}}
$$

where $S[\mathrm{~V} / \mathrm{K}]$ is the Seebeck coefficient, $\sigma[\mathrm{S} / \mathrm{m}]$ is the electrical conductivity, $\kappa_{\text {tot }}[\mathrm{W} / \mathrm{m} \mathrm{K}]$ is the thermal conductivity, $\kappa_{\mathrm{el}}$ and $\kappa_{\mathrm{ph}}$ are the carrier and phonon components of $\kappa_{\mathrm{tot}}$, respectively ${ }^{[23]}$. The product $S^{2} \sigma$ is called the electrical power factor (PF) and depends on the Seebeck coefficient and on the electrical conductivity. For convenience, dimensionless figure of merit $Z T$ is widely use to represent the efficiency of the n-type and p-type materials which compose a thermoelectric device. $Z T$ is directly determined multiplying $Z$ by the operating temperature. A thermoelectric material having a higher figure of merit ZT is more convenient, as it can carry out higher conversion power ${ }^{[24]}$, hence the search for higher electrical power factor and combined with a lower thermal conductivity is desirable for the optimal material performance.

$$
Z T=\frac{S^{2} \sigma}{\kappa_{e l}+\kappa_{p h}} T=\frac{P F}{\kappa_{t o t}} T
$$

Owing to thermoelectric materials low efficiency conversion, their applications have been limited to focused fields where the reliability rather than the cost is a major consideration ${ }^{[25]}$. In this assessment, researchers have focused their efforts to maximize the thermoelectric materials figure of merit $Z T$, because it is directly related to the equation for Carnot efficiency ${ }^{[26]}$ :

$$
\eta=\frac{T_{h}-T_{c}}{T_{h}} \frac{\sqrt{1+Z T}-1}{\sqrt{1+Z T}+T_{c} / T_{h}}
$$


where $\eta$ defines the Carnot efficiency, $Z T$ is the figure of merit of the material at the corresponding temperature, $T_{h}$ the temperature of the heat source (hot end), and $T c$ is the temperature of the heat sink or cold end. To illustrate this, if a material with a $Z T$ of 1 , with a heat source of $1000 \mathrm{~K}$, and a cold end of room temperature will yield an efficiency of $14 \%$, considering both materials have equal values of $Z T$ at the corresponding temperature. Figure 1-6 illustrates the conversion efficiencies for different $Z T$ values. In order to be competitive with traditional mechanical energy conversion systems, thermoelectric materials with $Z T$ values above 2 are ideal in order to promote practical applications $^{[27]}$.

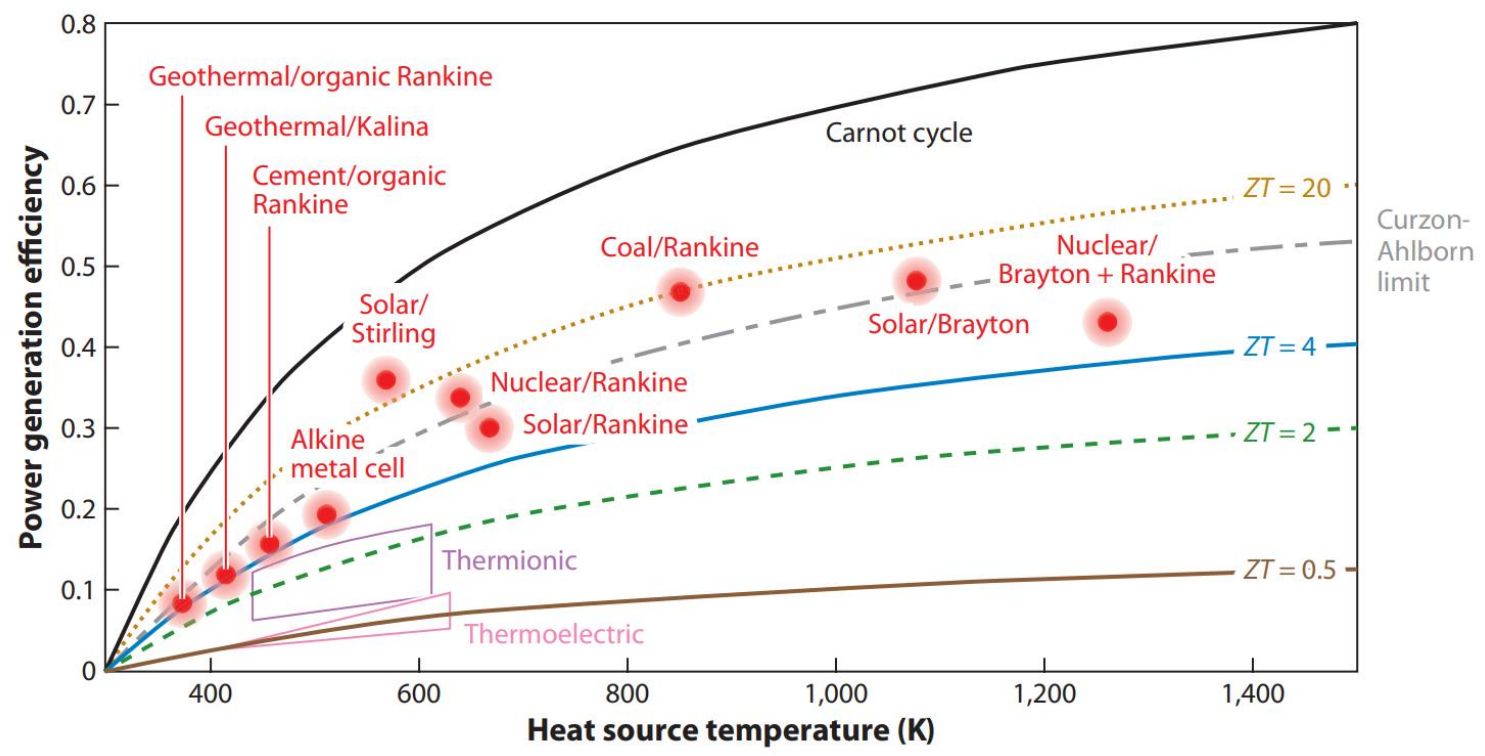

Figure 1-6: Thermoelectric energy conversion as a function of ZT at the setting of $T_{c}=300 \mathrm{~K}$, and efficiency for conventional thermal engines. ${ }^{[28]}$

Nonetheless, it is necessary to analyze in detail the conditions and assumptions when using this equation to express the efficiency of a thermoelectric device, since there are more conditions affecting the efficiency that only materials performance, such conditions can be found in literature ${ }^{[29]}$. Additionally, electrical and thermal resistances of the contacts and electrodes are neglected and constant ZT values are assumed for the Carnot equation ${ }^{[30]}$. To succeed in the goal of the development of thermoelectric materials with superior performance, the physical parameters 
related with the $Z T$ must be tailored. Nonetheless, it is highly important to first recognize the contributions of the transport properties $S, \sigma$, and $\kappa$, since they are intrinsically related upon one another predominantly as a function of the carrier concentration and carrier mobility ${ }^{[31]}$. For instance, the Seebeck coefficient for a semiconductor with parabolic band dispersion in relation with the carrier concentration is given by ${ }^{[32]}$ :

$$
S=\frac{8 \pi^{2} k_{b}^{2}}{3 e h^{2}}(m)(T)\left(\frac{\pi}{3 n}\right)^{2 / 3}
$$

where $k_{b}$ is the Boltzmann constant, $e$ the electron charge, $h$ the Planck constant, and $\mathrm{m}$ the density of states effective mass of carriers, and $n$ the carrier concentration. Consequently, a material with a high Seebeck coefficient must own a low carrier concentration, which is usually find in semiconductors or insulators ${ }^{[33]}$. Conversely, the electrical conductivity is given by:

$$
\sigma=n e \mu
$$

where $n$ is the carrier concentration, $e$ is the electron charge, and $\mu$ the carrier mobility. Empirical relations exist between carrier concentration and mobility ${ }^{[34]}$. Thus, to obtain a large $\sigma$ the carrier concentration and mobility should be as high as possible in order to attain a great electrical power factor. Moreover, $\kappa_{\text {tot }}$ holds two core contribution from $\kappa_{\mathrm{el}}$ and $\kappa_{\mathrm{ph}}, \kappa_{\mathrm{ph}}$ is the phonon contribution and has little dependence on the carrier concentration $n$, contrariwise $\kappa_{\mathrm{el}}$ is the carrier contribution and is directly influenced by $n$ through the Wiedemann-Franz relation, as given by the equation ${ }^{[35]}$ :

$$
\kappa_{e l}=L \sigma T=L n e \mu T
$$

where $\mathrm{L}$ is the Lorenz number. Subsequently, there is a direct consequence by increasing the electrical conductivity the material will have a proportional increase in the thermal conductivity. Thus, the contribution of the $\kappa_{\mathrm{ph}}$ is obtained by deducting the role of the $\kappa_{\mathrm{el}}$ to the overall value of 
$\kappa_{\text {tot. }}$ Since $\kappa_{\text {ph }}$ has little to none dependence in carrier concentration, the lattice contribution it is one aspect to optimize without suffer from low electrical power factor ${ }^{[33]}$ because is the only parameter not determined by the electronic structure. In most cases, thermoelectric parameters such as electrical conductivity and Seebeck coefficient cannot be optimized independently due to their relation with the electronic structure of the material ${ }^{[36]}$. Additionally, the kinetic theory provides a good approximation for the lattice thermal conductivity.

$$
\kappa_{p h}=1 / 3 C_{v} l v_{s}
$$

where $C_{v}$ is the specific heat a constant volume, $v_{s}$ is the velocity of the sound, and $l$ is the mean free path of the phonons. Values for $C_{v}$, and $v_{s}$ are independent variables for typical materials at high temperature. Consequently, $\kappa_{\mathrm{ph}}$ is essentially determined by the mean free path of the phonons at higher temperatures ${ }^{[9]}$. Although the power factor could be increased by tunning the doping concentration, thus, modifying the amount present of charge carriers in the material, reducing $\kappa_{\text {tot }}$ and $\kappa_{\mathrm{ph}}$ is considerable more challenging, in particular for $\kappa_{\mathrm{ph}}$, because is determined by the structure, rigidity, atomic masses, and other characteristics of the lattice ${ }^{[37]}$.

Figure 1-7 depicts the thermoelectric properties $S, \sigma$, and $\kappa$ for a classical semiconductor as a function of the carrier concentration. The optimal doping level to attain the best carrier concentration could lead to maximize the ZT of the material. Additionally, reducing $\kappa_{\mathrm{ph}}$ lead to a further decrease of $\kappa_{\text {tot }}$ and a larger Seebeck coefficient. Such values can be adjusted by controlling the doping level ${ }^{[33]}$. 

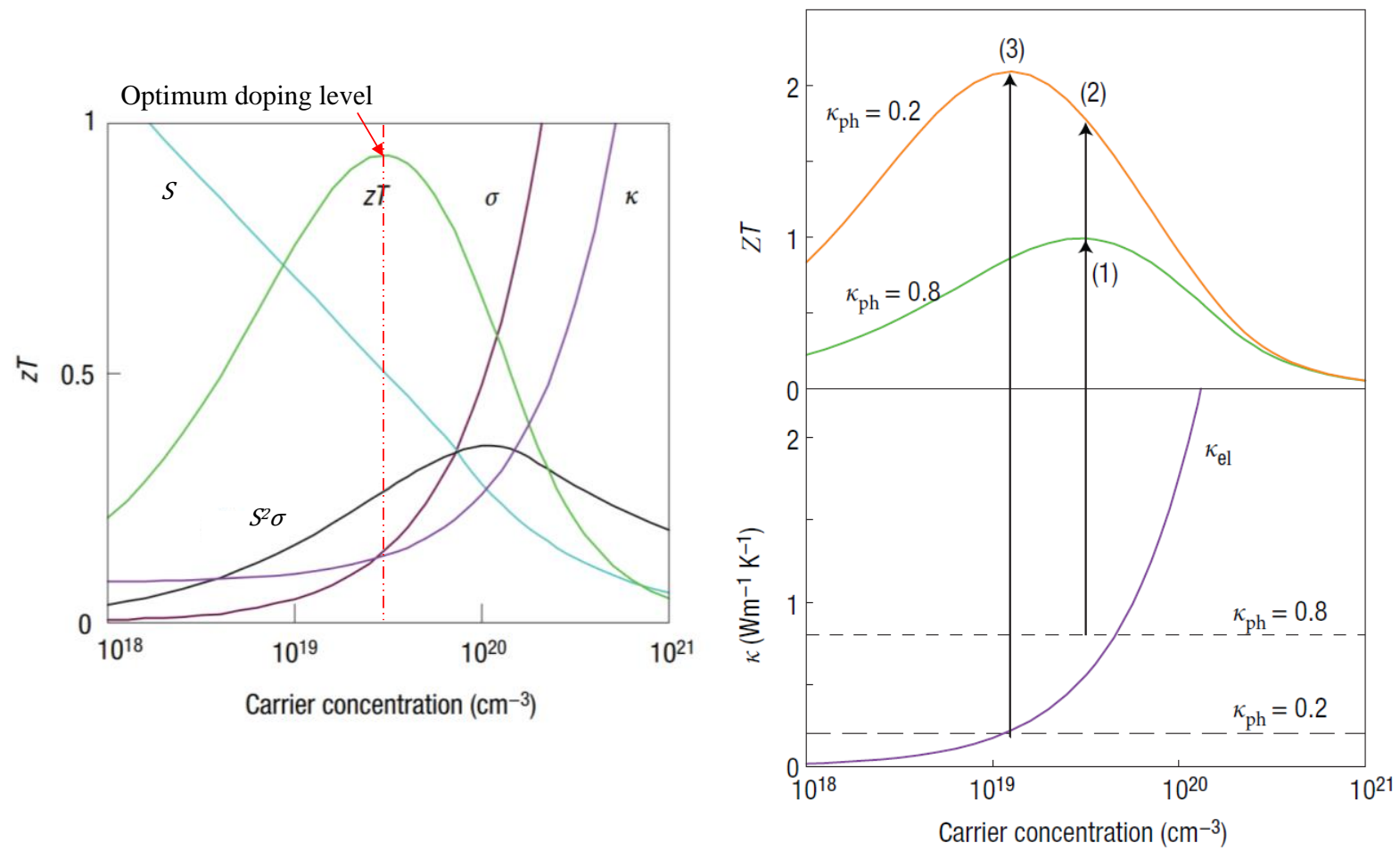

Figure 1-7: Optimizing ZT through carrier concentration tuning: Left: Maximizing the efficiency (ZT) of a thermoelectric involves finding the middle ground of thermal conductivity and Seebeck coefficient with electrical conductivity. Right: Reducing the lattice thermal conductivity leads to a dual benefit for the thermoelectric figure of merit, lowering the thermal conductivity allows the carrier concentration to be reduced, leading to both a decrease in $\kappa_{e l}$ and a larger $S^{[15]}$.

\section{5 Strategies to optimize thermoelectric properties}

Given the necessity to develop outstanding commercial capabilities for thermoelectric generators to expand, gain a competitive advantage, and most notably to improve the efficiency, current materials require an integration of multiple successful techniques to improve performance. In this assessment, increasing the power factor by band engineering has been reported to exploit the performance of the electrical power factor by utilizing electron energy filtering, nanostructuring, highly mismatched electronic doping, and modulating doping. In addition, allowing for different sources of scattering on all relevant length scales (from atomic-scale lattice disorder and nanoscale to mesoscale grain boundaries $\left.{ }^{[38]}\right)$, it is possible to achieve a maximum reduction in lattice thermal conductivity and a large enhancement in the thermoelectric 
performance $^{[39]}$, as shown in Figure 1-8. Such state-of-the-art thermoelectric materials are commonly referenced as nanocomposites because they compromise nanostructures from fine grains, dispersed particles, nanoinclusions to atomic defects ${ }^{[40]}$. Furthermore, a high vacancy concentration is reported to reduce the lattice thermal conductivity of a semiconductor ${ }^{[41]}$. Additionally, point defects could have a positive influence generating potential barriers at the grain boundaries to enhance $S^{[42]}$, since low energy carriers contribute negatively to $S^{[43]}$, thus filtering these low energy carriers improve $S$. This nanostructuration synthesis could be achieved by utilizing superlattices, modulation doping, surface doping in a bottom-up engineering method to control composition, size, shape, and phase of the material ${ }^{[44]}$.

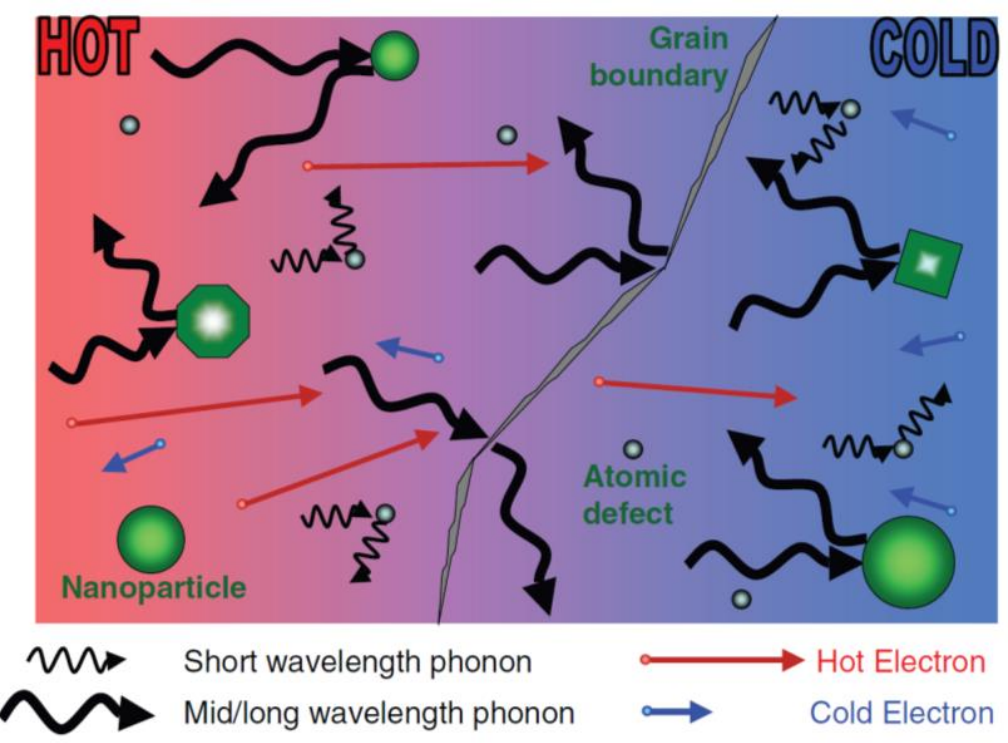

Figure 1-8: Schematic diagram illustrating various phonon scattering mechanisms within a thermoelectric material, along with electronic transport of hot and cold electrons. Atomic scale defects in alloys scatter phonons due to differences in mass or due to generation of strain fields, additionally the inclusion of nanoparticles, significantly reduce lattice thermal conductivity by the additional scattering of mid-and long-wavelength phonons ${ }^{[45]}$.

Modulation doping is one technique discussed as an effective way to increase the ZT by the means of carrier mobility enhancement relative to uniform doping because it avoids ionized impurity scattering. Modulation doping compromise two phase composite materials made of undoped and heavily doped phases, such spatial separation of carriers and impurity scattering 
enable a higher carrier mobility without deteriorating the Seebeck coefficient ${ }^{[33]}$. Also, the combination of a super lattice arrangement and modulation doping provide energy filtering effects as an effective way to promote a better performance in ZT by the means of a Seebeck coefficient and enhancement of carrier mobility ${ }^{[46]}$. Moreover, alignment of the plate-like grains in anisotropic materials is reported as a feasible approach to increase carrier mobility, commonly the layered structures of these materials have better electrical conductivity in the in-plane than the out-plane overshadowing the ratio related to the thermal conductivity. Methods such as spark plasma sintering, hot pressing, and magnetic processing ${ }^{[47] .}$ More importantly, in thermoelectric ceramic materials the most promising route to optimize the $\mathrm{ZT}$ value for most materials has been cationic substitution $^{[48]}$.

\section{6 Thermoelectric materials}

Thermoelectric materials are considered as an alternative that allow thermal losses to be recovered and directly convert it into electrical energy using thermoelectric generators when a temperature difference is placed through the device ${ }^{[49]}$. Heavily doped semiconductors with behavior similar to the metals make for the best thermoelectric materials, as insulators have high electrical resistivity and metals exhibit low Seebeck coefficient ${ }^{[50]}$. The majority of novel thermoelectric materials displaying a high $Z T$ contain heavy elements such as $\mathrm{Pb}, \mathrm{Sb}, \mathrm{Te}$, or $\mathrm{Bi}^{[51]}$. In addition SiGe alloys have been used for a long time in thermoelectric modules either as a ptype $^{[52]}$ or as an n-type material ${ }^{[53]}$. Recently, SnSe layered crystals have been reported with a unprecedented $Z T$ value of 2.6 along the $b$ axis of the orthorhombic unit cell ${ }^{[54]}$. Tan et al. ${ }^{[33]}$ have summarized chronologically the most recent production of novel thermoelectric materials by ZT performance and operation temperature, as shown in Figure 1-9. 


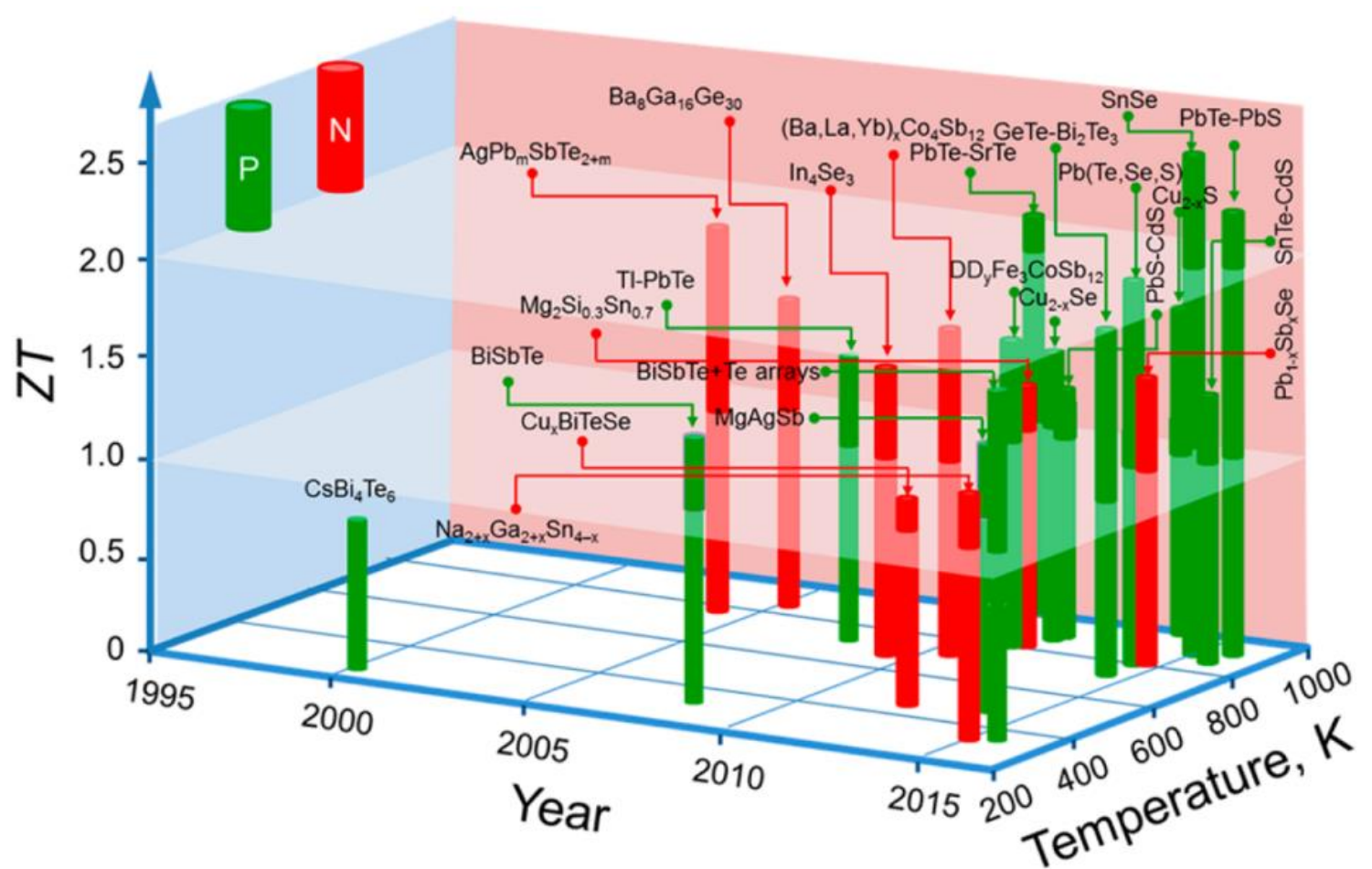

Figure 1-9: Development of bulk thermoelectric materials: the thermoelectric figure-of-merit ZT as a function of temperature and year. Green cylinders represent the p-type materials, while red cylinders represent the n-type ones. ${ }^{[33]}$

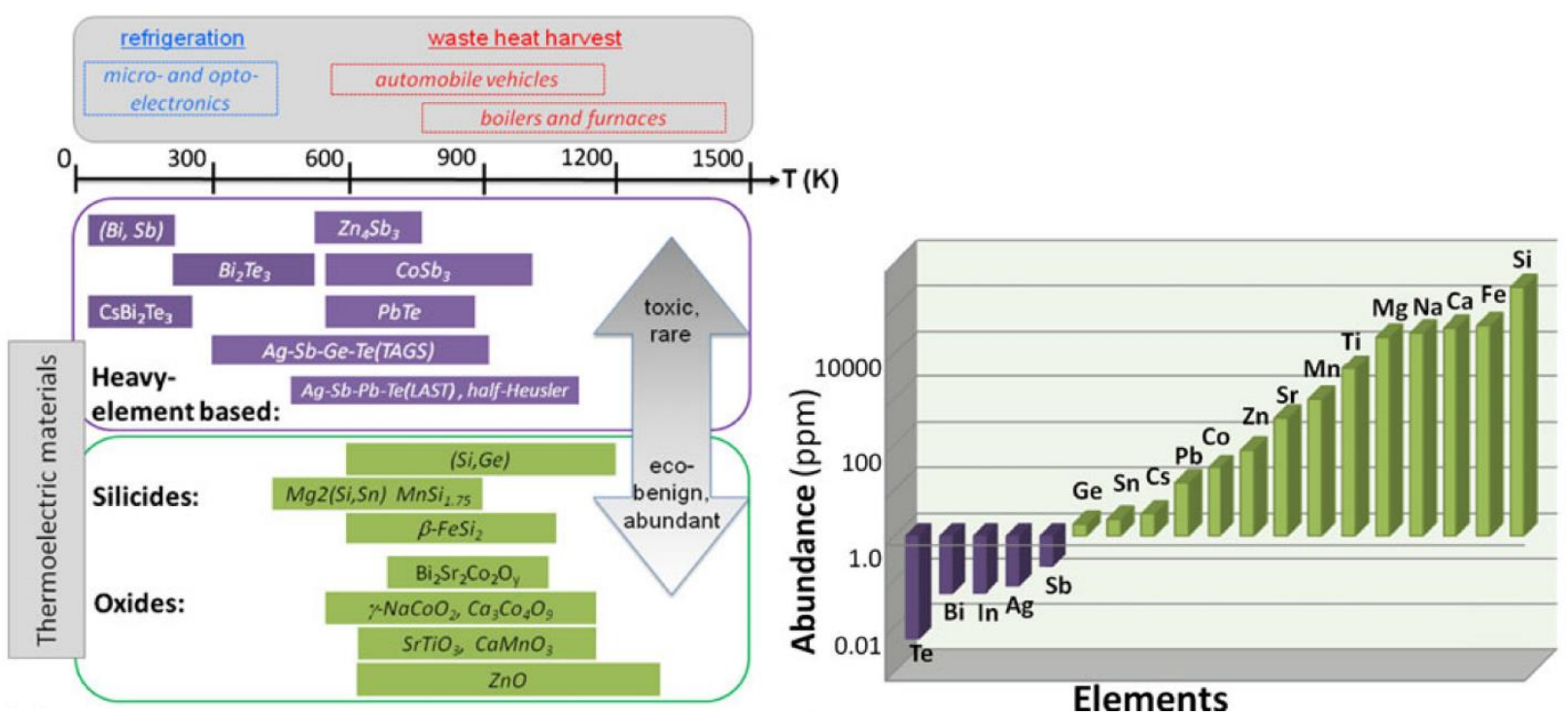

Figure 1-10: Left: Schematic comparison of various thermoelectric (TE) materials for applications of waste heat harvest and refrigeration, in terms of the temperature range of operation and the abundance and environmental friendliness of constituent elements. Rigth: The abundance of elements used in TE materials, the elements represented by darkcolored columns are $<1$ ppm. ${ }^{[51]}$ 
Although these materials developed have a performance capable of competing in efficiency with some conventional heat recovery technologies due to their high ZT, they own several inherent disadvantages for their applications in thermoelectric devices. First, the low chemical and thermal stability of such materials could impact in their ability to perform well in a large temperature gradient in air. Second, the cost of raw materials for the compounds aforementioned in Figure 1-9 are not suitable for most application. Finally, due to the lack of abundant sources, for instance in BiTe both materials are scarce, and the toxicity of the elements used for these compounds, they hold a higher risk if utilized for thermoelectric device applications and could be hazardous for the environment.

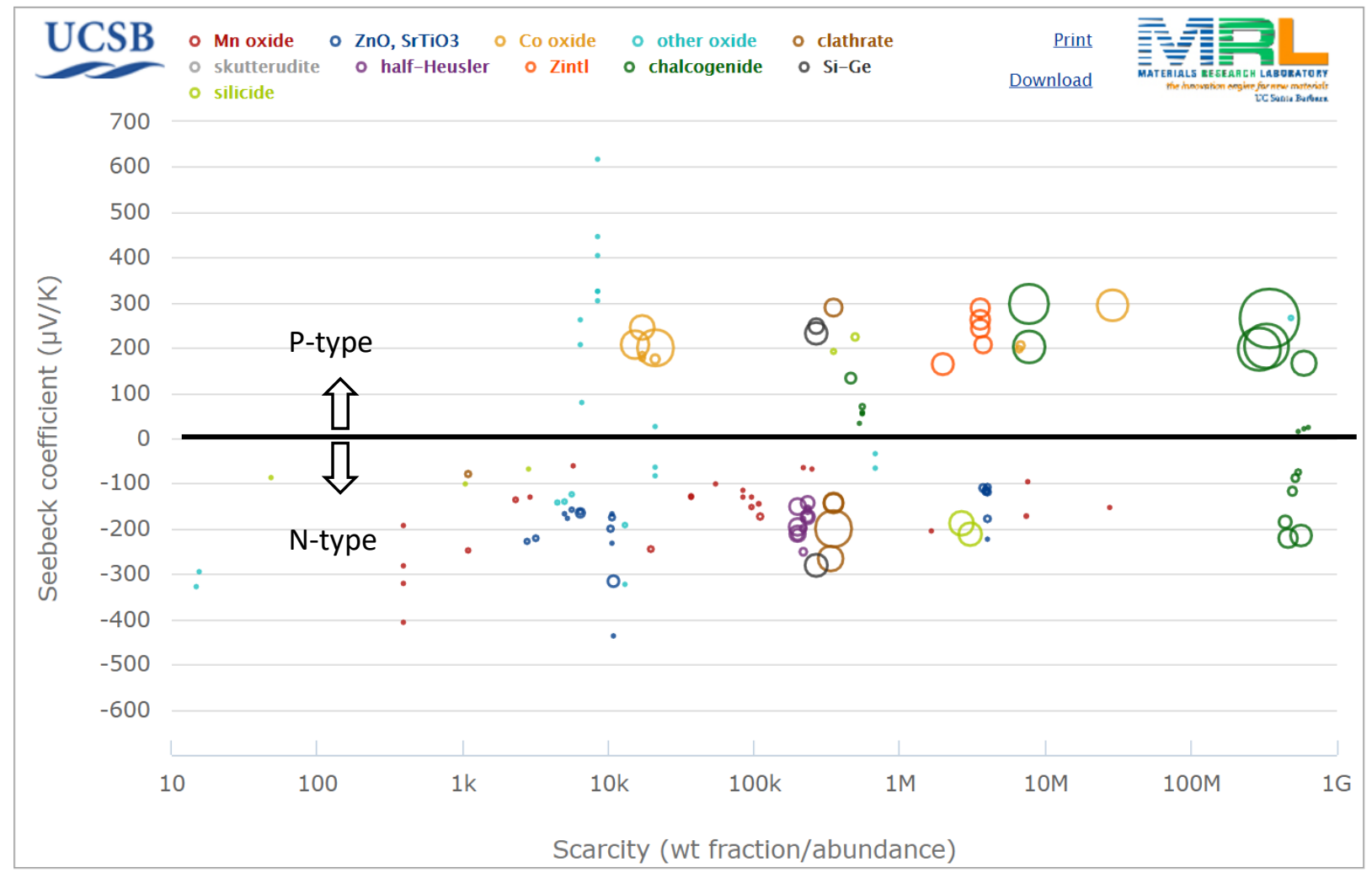

Figure 1-11: Thermoelectric performance of different material families in relation with the scarcity of the constituents. Marker size is related with the dimensionless figure of merit ZT of the material. ${ }^{[55]}$

In contrast, oxide thermoelectric materials provide desirable characteristics as constituent materials for commercial thermoelectric devices. Oxide materials are compromised from highly 
abundant elements that are non-toxic which are chemical and thermal stable at high temperatures.

Figure 1-10 displays the thermoelectric materials at different temperature range applications in terms of toxicity and abundancy ${ }^{[51]}$. Due to their abundance and good thermoelectric performance (Figure 1-11), oxides and silicides make for the best selection of material for thermoelectric applications at high temperatures with the additional benefit of having cheap raw materials and for being environmentally friendly compared to conventional thermoelectric materials.

From the multiple promising p-type oxide materials candidates, thermoelectric modules composed of layered cobaltites have been studied since the discovery in 1997 of the large thermoelectric power in the $\mathrm{Na}_{x} \mathrm{CoO}_{2}$ single crystal ${ }^{[56]}$. The crystal structure of these layered cobaltites include the $\mathrm{CdI}_{2}$-type $\mathrm{CoO}_{2}$ block in common (responsible for good electrical conductivity and key component for good Seebeck coefficient), which alternately stacks with various block layers along the $\mathrm{c}$ axis (responsible for lattice thermal conduction). Several block layers for this compound are displayed in Figure 1-12 $2^{[57]}$. These thermoelectric materials presents an exceptional performance due to their layered nature, such characteristic reach a great performance as a result of the dedicated layers where the electric and the thermal conductivity flow in different paths in the crystal ${ }^{[58]}$.
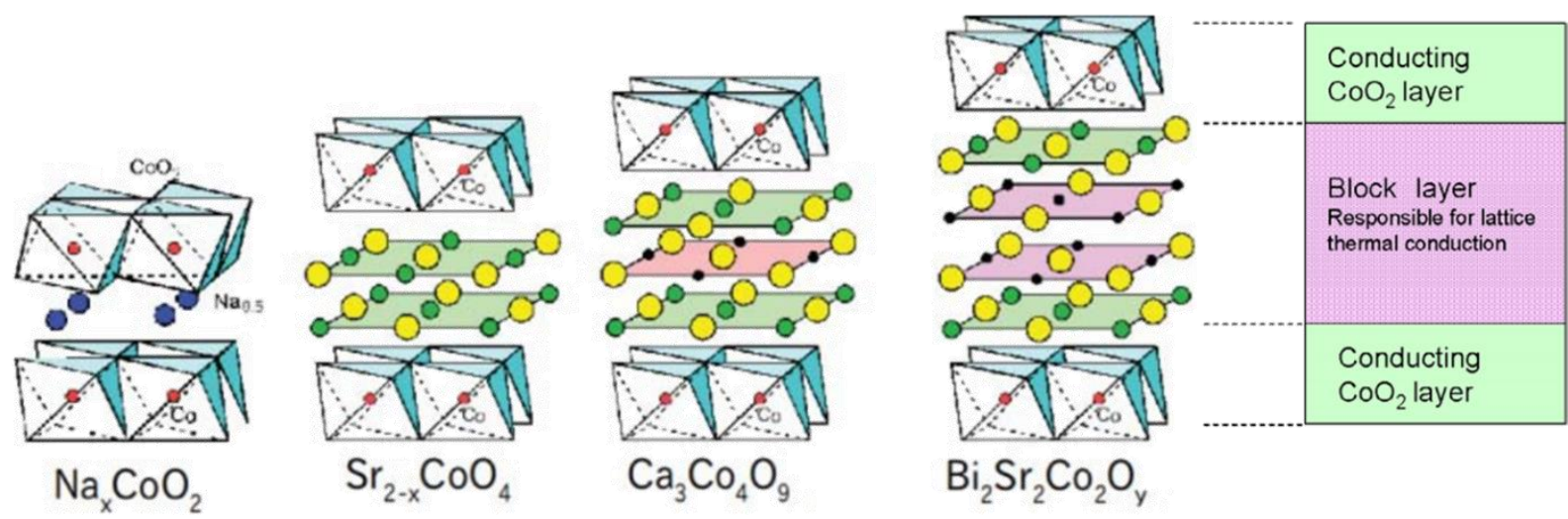

Figure 1-12: Crystal structures of the layered cobalt oxides. $\mathrm{CoO}_{2}$ block in common (responsible for good electrical conductivity and key component for good Seebeck coefficient), which alternately stacks with various block layers along the c axis (responsible for lattice thermal conduction). ${ }^{[57]}$ 
Figure 1-13 shows that all layered cobalt oxides are found to be exceptional thermoelectric materials at high temperature. It is evident that the performance given by the ZT values of the layered cobalt oxides are far superior to the p-type $\mathrm{PbTe}$ and $\mathrm{Si}_{1-\mathrm{x}} \mathrm{Ge}_{\mathrm{x}}$ above $600{ }^{\circ} \mathrm{C}$. Notably, layered cobaltite Bi-Sr-Co-O system shows a ZT of $1.19^{[59]}$, however the ZT for polycrystalline ceramics of this compound decreases badly due to their higher resistivity ${ }^{[60]}$. Additionally, the single crystal performance of $\mathrm{Ca}_{2} \mathrm{Co}_{2} \mathrm{O}_{5}$ and $\mathrm{Ca}_{3} \mathrm{Co}_{4} \mathrm{O}_{9}$ have been reported to own a $Z T$ of 1.2 at $873^{[61]}$ and 0.87 at $973^{[62]}$, respectively. Although $\mathrm{Ca}_{2} \mathrm{Co}_{2} \mathrm{O}_{5}$ has a large $Z T, \mathrm{Ca}_{3} \mathrm{Co}_{4} \mathrm{O}_{9}$ has been more widely used for thermoelectric applications ${ }^{[48]}$.

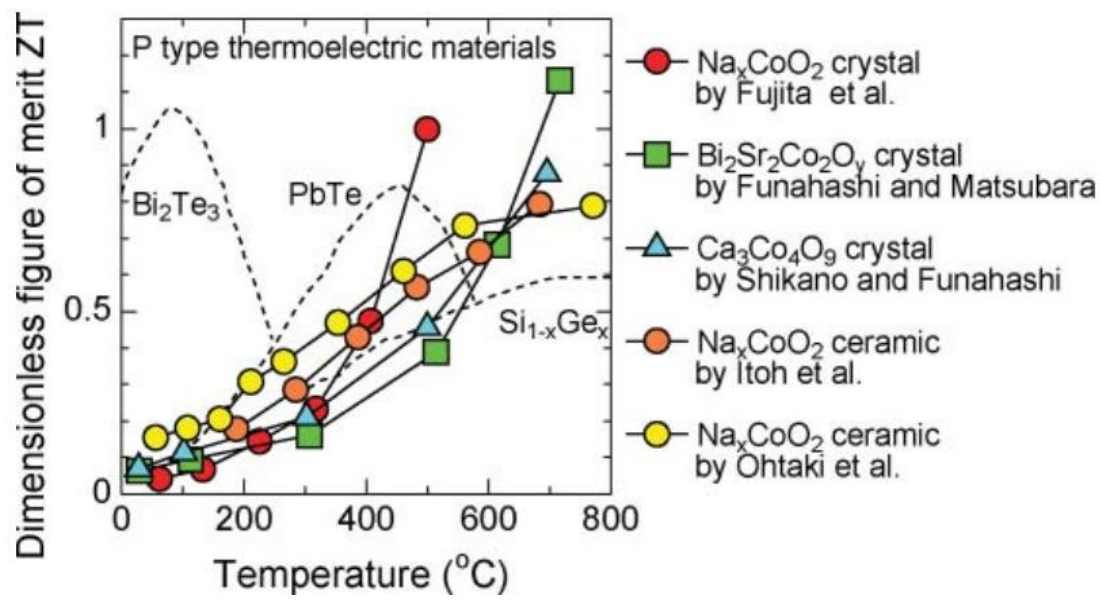

Figure 1-13: Dimensionless figure of merit ZT of the layered cobalt oxides comparing to conventional thermoelectric material. ${ }^{[57]}$

Nonetheless, single crystal materials are difficult and more expensive to fabricate than the polycrystalline form. Furthermore, the layered structure anisotropy for bulk ceramics in these materials is a challenging assignment in order to reach the single crystal performance. ${ }^{[63][64]}$ Most of the reported literature deal in a big time to improve the electrical and thermal properties at the same time, which is why polycrystalline performance still below with an average value around 30$50 \%$ compared to the undoped single crystal value ${ }^{[48]}$. 


\section{7 Summary}

To sum up, there is an enormous worldwide potential to take advantage of the energy lost after its conversion from the main utilization, especially the energy lost as waste heat. Adopting technologies to harvest waste heat and reutilize this energy source could make processes more efficient and provide economic and environmental benefits by reducing fuel consumption. Thermoelectric (TE) technology could provide with an alternative to harvest the excessive amount of energy lost as waste heat and convert it directly to electrical power due to the Seebeck effect, which was discovered by Thomas Seebeck in 1821. Although this phenomenon was discovered almost 200 years ago, it is still challenging for researchers to improve the performance due to the interconnected properties. In this assessment, different research approaches have been demonstrated to improve individual parameters, however, to obtain high performance TE materials require an integration of multiple successful techniques to improve the overall performance. Some conventional materials already possess ZT larger than one but those have characteristics not goodfitted for commercial applications. Thus, it is indispensable to work with TE oxides which are better suited economically, environmentally, and property-wise for these applications. Layered oxides have been showed to be a good candidate for this application due to its high thermal stability in the air at high temperatures, low cost, lightweight, and non-toxicity. However, TE energy conversion efficiency of polycrystalline TE such as $\mathrm{Ca}_{3} \mathrm{Co}_{4} \mathrm{O}_{9-\delta}$ remains low and accounts to $~ 30-$ $60 \%$ of the single crystals. To attain a high ZT, carrier mobility and carrier concentration need to be optimized at the same time to improve the electrical transport properties and the material should also incorporate phonon scattering to reduce the thermal conductivity. Thus, in the next section, an overview of the recent developments in this $\mathrm{Ca}_{3} \mathrm{Co}_{4} \mathrm{O}_{9-\delta}$ thermoelectric material is offered. 


\section{References}

1. $\quad$ Agency, I.E., World energy balances: Overview (2017 edition) 2017.

2. Administration, U.S.E.I., International Energy Outlook 2016: With Projections to 2040. 2016, Government Printing Office.

3. Laboratory, L.L.N., Estimated U.S. Energy Consumption in 2018 2018. Retrieved from: https://flowcharts.llnl.gov/content/assets/docs/2018_United-States_Energy.pdf

4. U.S., D.o.E., Quadrennial technology review: An assessment of energy technologies and research Opportunities 2015. p. 1-505.

5. Viswanathan, V.V., R.W. Davies, and J.D. Holbery, Opportunity analysis for recovering energy from industrial waste heat and emissions. 2006, Pacific Northwest National Lab.(PNNL), Richland, WA (United States).

6. Brückner, S., et al., Industrial waste heat recovery technologies: An economic analysis of heat transformation technologies. Applied Energy, 2015. 151: p. 157-167.

7. Lu, H., L. Price, and Q. Zhang, Capturing the invisible resource: Analysis of waste heat potential in Chinese industry. Applied Energy, 2016. 161: p. 497-511.

8. Seebeck, T.J., Magnetische polarisation der metalle und erze durch temperatur-differenz. Vol. 70. 1895: W. Engelmann.

9. Tritt, T.M., Thermoelectric Phenomena, Materials, and Applications. Annual Review of Materials Research, 2011. 41(1): p. 433-448.

10. Peltier, J., Nouvelles expériences sur la caloricité des courants électrique. Ann. Chim. Phys, 1834. 56(371): p. 371-386.

11. Starling, S.G., Demonstration of Peltier and Thomson Effects. Nature, 1911. 85: p. 512.

12. Thomson, W., On the Dynamical Theory of Heat. Part V. Thermo-electric Currents. Transactions of the Royal Society of Edinburgh, 1857. 21(1): p. 123-171.

13. Penrose, C.B., Thermo-Electricity. Peltier and Thomson Effects. Proceedings of the American Academy of Arts and Sciences, 1881. 17: p. 39-46.

14. Altenkirch, E., Elektrothermische Kälteerzeugung und reversible elektrische Heizung. Physikalische Zeitschrift, 1911. 12: p. 920-924.

15. Snyder, G.J. and E.S. Toberer, Complex thermoelectric materials. Nature Materials, 2008. 7: p. 105.

16. Li, J.-F., et al., High-performance nanostructured thermoelectric materials. Npg Asia Materials, 2010. 2: p. 152.

17. Polozine, A., S. Sirotinskaya, and L. Schaeffer, History of development of thermoelectric materials for electric power generation and criteria of their quality. Materials Research, 2014. 17: p. 1260-1267.

18. Xi, H., L. Luo, and G. Fraisse, Development and applications of solar-based thermoelectric technologies. Renewable and Sustainable Energy Reviews, 2007. 11(5): p. 923-936.

19. Awaya, H.I., et al., Thermoelectric power conversion in space. Mechanical Engineering, 1990. 112(9): p. 75.

20. Ritz, F. and C.E. Peterson. Multi-mission radioisotope thermoelectric generator (MMRTG) program overview. in 2004 IEEE Aerospace Conference Proceedings (IEEE Cat. No.04TH8720). 2004.

21. Bell, L.E., Cooling, Heating, Generating Power, and Recovering Waste Heat with Thermoelectric Systems. Science, 2008. 321(5895): p. 1457. 
22. Champier, D., Thermoelectric generators: A review of applications. Energy Conversion and Management, 2017. 140: p. 167-181.

23. Ohtaki, M., Recent aspects of oxide thermoelectric materials for power generation from mid-to-high temperature heat source. Journal of the Ceramic Society of Japan, 2011. 119(1395): p. 770-775.

24. Enescu, D. and E.O. Virjoghe, A review on thermoelectric cooling parameters and performance. Renewable and Sustainable Energy Reviews, 2014. 38: p. 903-916.

25. Zheng, X.F., et al., A review of thermoelectrics research - Recent developments and potentials for sustainable and renewable energy applications. Renewable and Sustainable Energy Reviews, 2014. 32: p. 486-503.

26. Kanatzidis, M.G., Advances in thermoelectrics: From single phases to hierarchical nanostructures and back. MRS Bulletin, 2015. 40(8): p. 687-695.

27. Chen, Z.-G., et al., Nanostructured thermoelectric materials: Current research and future challenge. Progress in Natural Science: Materials International, 2012. 22(6): p. 535-549.

28. Shakouri, A., Recent Developments in Semiconductor Thermoelectric Physics and Materials. Annual Review of Materials Research, 2011. 41(1): p. 399-431.

29. Kim, H.S., et al., Relationship between thermoelectric figure of merit and energy conversion efficiency. Proceedings of the National Academy of Sciences, 2015. 112(27): p. 8205.

30. Zebarjadi, M., et al., Perspectives on thermoelectrics: from fundamentals to device applications. Energy \& Environmental Science, 2012. 5(1): p. 5147-5162.

31. Hamid Elsheikh, M., et al., A review on thermoelectric renewable energy: Principle parameters that affect their performance. Renewable and Sustainable Energy Reviews, 2014. 30: p. 337-355.

32. Cutler, M., J.F. Leavy, and R.L. Fitzpatrick, Electronic Transport in Semimetallic Cerium Sulfide. Physical Review, 1964. 133(4A): p. A1143-A1152.

33. Tan, G., L.-D. Zhao, and M.G. Kanatzidis, Rationally Designing High-Performance Bulk Thermoelectric Materials. Chemical Reviews, 2016. 116(19): p. 12123-12149.

34. Hilsum, C., Simple empirical relationship between mobility and carrier concentration. Electronics Letters, 1974. 10(13): p. 259-260.

35. Franz, R. and G. Wiedemann, Ueber die Wärme-Leitungsfähigkeit der Metalle. Annalen der Physik, 1853. 165(8): p. 497-531.

36. Sootsman, J.R., D.Y. Chung, and M.G. Kanatzidis, New and Old Concepts in Thermoelectric Materials. Angewandte Chemie International Edition, 2009. 48(46): p. 8616-8639.

37. Dmitriev, A.V. and I.P. Zvyagin, Current trends in the physics of thermoelectric materials. Physics-Uspekhi, 2010. 53(8): p. 789-803.

38. He, J., M.G. Kanatzidis, and V.P. Dravid, High performance bulk thermoelectrics via a panoscopic approach. Materials Today, 2013. 16(5): p. 166-176.

39. Biswas, K., et al., High-performance bulk thermoelectrics with all-scale hierarchical architectures. Nature, 2012. 489: p. 414.

40. Liu, W., et al., Recent advances in thermoelectric nanocomposites. Nano Energy, 2012. 1(1): p. 42-56.

41. Qiu, B., et al., Molecular dynamics simulations of lattice thermal conductivity and spectral phonon mean free path of PbTe: Bulk and nanostructures. Computational Materials Science, 2012. 53(1): p. 278-285. 
42. Kishimoto, K., M. Tsukamoto, and T. Koyanagi, Temperature dependence of the Seebeck coefficient and the potential barrier scattering of n-type PbTe films prepared on heated glass substrates by rf sputtering. Journal of Applied Physics, 2002. 92(9): p. 5331-5339.

43. Zhao, X., X.-h. Zhu, and R.-z. Zhang, Evaluation of energy filtering effect from first principles calculations. physica status solidi (a), 2016. 213(12): p. 3250-3253.

44. Ortega, S., et al., Bottom-up engineering of thermoelectric nanomaterials and devices from solution-processed nanoparticle building blocks. Chemical Society Reviews, 2017. 46(12): p. 3510-3528.

45. Vineis, C.J., et al., Nanostructured Thermoelectrics: Big Efficiency Gains from Small Features. Advanced Materials, 2010. 22(36): p. 3970-3980.

46. Neophytou, N. and M. Thesberg, Modulation doping and energy filtering as effective ways to improve the thermoelectric power factor. Journal of Computational Electronics, 2016. 15(1): p. 16-26.

47. Koumoto, K., et al., Thermoelectric Ceramics for Energy Harvesting. Journal of the American Ceramic Society, 2013. 96(1): p. 1-23.

48. Fergus, J.W., Oxide materials for high temperature thermoelectric energy conversion. Journal of the European Ceramic Society, 2012. 32(3): p. 525-540.

49. Twaha, S., et al., A comprehensive review of thermoelectric technology: Materials, applications, modelling and performance improvement. Renewable and Sustainable Energy Reviews, 2016. 65: p. 698-726.

50. Amollo, T.A., et al., Graphene for Thermoelectric Applications: Prospects and Challenges Critical Reviews in Solid State and Materials Sciences, 2018. 43(2): p. 133-157.

51. He, J., Y. Liu, and R. Funahashi, Oxide thermoelectrics: The challenges, progress, and outlook. Journal of Materials Research, 2011. 26(15): p. 1762-1772.

52. Joshi, G., et al., Enhanced Thermoelectric Figure-of-Merit in Nanostructured p-type Silicon Germanium Bulk Alloys. Nano Letters, 2008. 8(12): p. 4670-4674.

53. Wang, X.W., et al., Enhanced thermoelectric figure of merit in nanostructured n-type silicon germanium bulk alloy. Applied Physics Letters, 2008. 93(19): p. 193121.

54. Zhao, L.-D., et al., Ultralow thermal conductivity and high thermoelectric figure of merit in SnSe crystals. Nature, 2014. 508: p. 373.

55. Gaultois, M.W., et al., Data-Driven Review of Thermoelectric Materials: Performance and Resource Considerations. Chemistry of Materials, 2013. 25(15): p. 2911-2920.

56. Terasaki, I., Y. Sasago, and K. Uchinokura, Large thermoelectric power in $\mathrm{NaCo}_{2} \mathrm{O}_{4}$ single crystals. Physical Review B, 1997. 56(20): p. R12685-R12687.

57. Terasaki, I., et al., Novel thermoelectric properties of complex transition-metal oxides. Dalton Transactions, 2010. 39(4): p. 1005-1011.

58. Terasaki, I. Thermoelectric materials in layered transition-metal oxides. in ICT 2005. 24th International Conference on Thermoelectrics, 2005. 2005.

59. Funahashi, R. and M. Shikano, $\mathrm{Bi}_{2} \mathrm{Sr}_{2} \mathrm{CO}_{2} \mathrm{O}_{y}$ whiskers with high thermoelectric figure of merit. Applied Physics Letters, 2002. 81(8): p. 1459-1461.

60. Funahashi, R., I. Matsubara, and S. Sodeoka, Thermoelectric properties of $\mathrm{Bi}_{2} \mathrm{Sr}_{2} \mathrm{Co}_{2} \mathrm{O}_{x}$ polycrystalline materials. Applied Physics Letters, 2000. 76(17): p. 2385-2387.

61. Funahashi, R., et al., An Oxide Single Crystal with High Thermoelectric Performance in Air. Japanese Journal of Applied Physics, 2000. 39(Part 2, No. 11B): p. L1127-L1129. 
62. Shikano, M. and R. Funahashi, Electrical and thermal properties of single-crystalline $\left(\mathrm{Ca}_{2} \mathrm{CoO}_{3}\right)_{0.7} \mathrm{CoO}_{2}$ with a $\mathrm{Ca}_{3} \mathrm{Co}_{4} \mathrm{O}_{9}$ structure. Applied Physics Letters, 2003. 82(12): p. 1851-1853.

63. Ito, M., et al., Synthesis of $\mathrm{Na}_{x} \mathrm{Co}_{2} \mathrm{O}_{4}$ thermoelectric oxides by the polymerized complex method. Scripta Materialia, 2003. 48(4): p. 403-408.

64. Saini, S., et al., Terbium Ion Doping in $\mathrm{Ca}_{3} \mathrm{Co}_{4} \mathrm{O}_{9}$ : A Step towards High-Performance Thermoelectric Materials. Scientific Reports, 2017. 7: p. 44621. 


\section{Chapter 2. Review of $\mathrm{Ca}_{3} \mathrm{Co}_{4} \mathrm{O}_{9}$ Thermoelectric Ceramics}

\section{1 Potential of thermoelectric oxide $\mathrm{Ca}_{3} \mathrm{Co}_{4} \mathrm{O}_{9}$}

Most of the processes in the industry that include energy conversion or require heat to operate suffer from a significant loss of energy in the form of waste heat. These thermal losses are the result of process inefficiencies and the inability of the existing process to recover and use the excess of energy. Therefore, there is a great opportunity to convert such wasted energy into an economic benefit, and at the same time, the prospect to improve the process and reduce the use of fuel resources. Thermoelectric materials are capable of directly convert such wasted heat into electricity. Additionally, because thermoelectric efficiency increases with the temperature difference, high temperature waste heat is a key factor to deliver a large temperature difference, thus, improving the conversion energy efficiency, given it could provide a heat source temperature greater than the heat sink temperature ${ }^{[1]}$.

Furthermore, given the necessity to develop thermoelectric materials capable of being stable at high temperature, oxides have shown a promising thermoelectric performance at large temperature differences, particularly, $\mathrm{Ca}_{3} \mathrm{Co}_{4} \mathrm{O}_{9}$ ceramic is reported to be a potential candidate for high temperature thermoelectric applications as a p-type material ${ }^{[2]}$, notably, displaying a high thermally stable in air up to $1199 \mathrm{~K}^{[3]}$ and above this temperature thermally decompose to $\mathrm{Ca}_{3} \mathrm{Co}_{2} \mathrm{O}_{6}$ and $\mathrm{Co}_{1-\mathrm{x}} \mathrm{Ca}_{\mathrm{x}} \mathrm{O}^{[4]}$. Some studies using $\mathrm{Ca}_{3} \mathrm{Co}_{4} \mathrm{O}_{9}$ as a thin film ${ }^{[5]}$ or as a SOFC cathode ${ }^{[6]}$ have been investigated but they will not be discussed in detail the present paper.

\section{2 Crystal structure of $\mathrm{Ca}_{3} \mathrm{Co}_{4} \mathrm{O}_{9}$}

The unit cell of $\mathrm{Ca}_{3} \mathrm{Co}_{4} \mathrm{O}_{9}$ has a monoclinic structure consisting of two misfit layered subsystems: the rock salt-type $\mathrm{Ca}_{2} \mathrm{CoO}_{3}$ layer serving as phonon scattering region to reduce the thermal conductivity and the $\mathrm{CdI} 2$-type $\mathrm{CoO}_{2}$ layer serving as electronic transport system to reduce 
the electrical resistivity ${ }^{[7]}$. The elementary charge-transport process in $\mathrm{Ca}_{3} \mathrm{Co}_{4} \mathrm{O}_{9}$ ceramics is reported to be owed to hopping of a hole from $\mathrm{Co}^{4+}$ to $\mathrm{Co}^{3+}{ }^{[8]}$. strong modulation and long bond distances in the $\mathrm{Ca}_{2} \mathrm{CoO}_{3}$ subsystem prevent electron hopping ${ }^{[9]}$, thus, $\mathrm{Ca}_{2} \mathrm{CoO}_{3}$ layer is an insulating media and can be regarded as a charge reservoir that supply charges to $\mathrm{CoO}_{2}$ layer ${ }^{[10]}$. Promoting a more aligned grain orientation is, therefore, imperative to enhance the transport properties of the highly anisotropic crystal structure of $\mathrm{Ca}_{3} \mathrm{Co}_{4} \mathrm{O}_{9}{ }^{[11]}$.

(a)

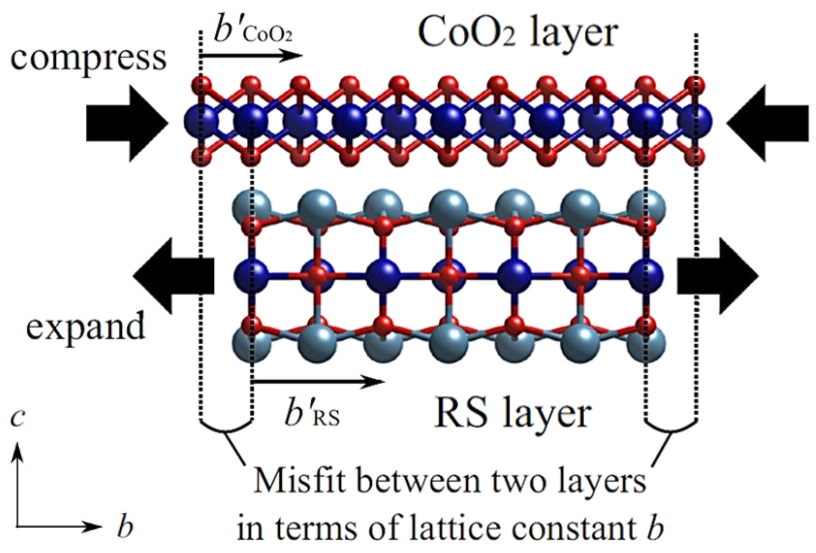

(c)

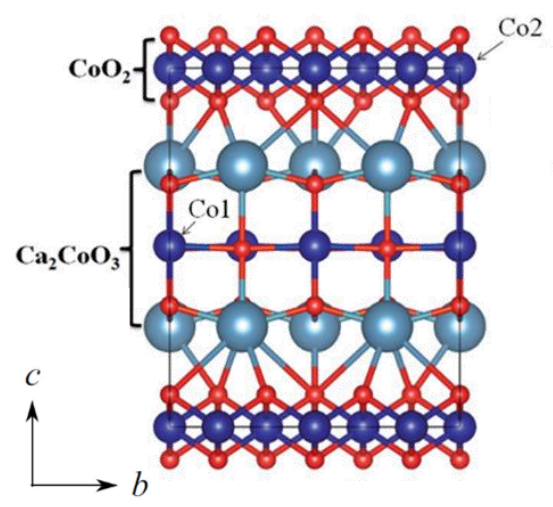

(b)
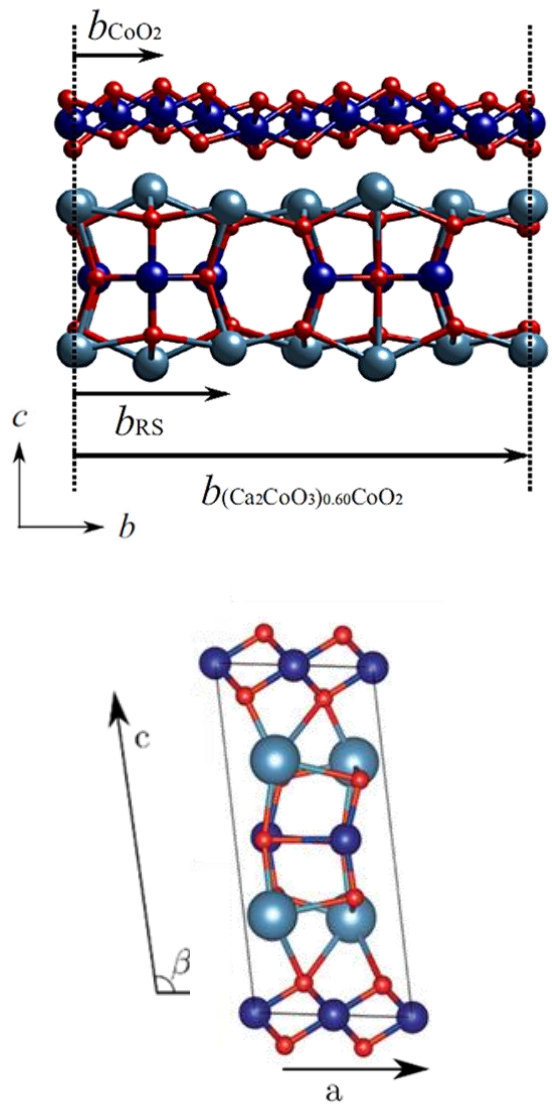

Figure 2-1: Representative schematic diagrams of (a) lattice misfit and $(b)$ the resulting displacements in the two layers. Light-blue, dark-blue, and red balls are Ca, Co, and O atoms, respectively ${ }^{[12]}$. (c) Diagram of the crystal structure of $\mathrm{Ca}_{3} \mathrm{Co}_{4} \mathrm{O}_{9}$ perpendicular to (a) the a axis and (b) the b axis. The Col and $\mathrm{Co} 2$ sites indicated refer to $\mathrm{Co}$ atoms from the $\mathrm{Ca}_{2} \mathrm{CoO}_{3}$ layer and the $\mathrm{CoO}_{2}$ layer, respectively ${ }^{[13]}$. 
Therefore, the aforementioned highly preferred orientation could be effective to increase the electrical conductivity due to the shorter carrier path and the reduction in the scattering of the carriers at the grain boundaries or defects ${ }^{[8]}$. The two monoclinic subsystems in the misfit oxide possess identical $a$-axes, $c$-axes, and $\beta$-angles parameters but different $b$-axis length, $b_{1}$ and $b_{2}$, for each monoclinic structure for the rock salt-type $\mathrm{Ca}_{2} \mathrm{CoO}_{3}$ layer and the CdI2-type $\mathrm{CoO}_{2}$ layer ${ }^{[14]}$. The typical cell parameter are $a \sim 4.83 \AA$, $c \sim 10.84 \AA$, $\beta \sim 98.14, b_{1} \sim 4.55 \AA$, and $b_{2} \sim 2.82 \AA^{[9]}$. Essentially, the anisotropic crystal structure of the $\mathrm{Ca}_{3} \mathrm{Co}_{4} \mathrm{O}_{9}$ is formed by a single layer of the CdI2-type $\mathrm{CoO}_{2}$, and a triple rock salt layer $[\mathrm{CaO}][\mathrm{CoO}][\mathrm{CaO}]$, as shown in Figure 2-1. These layers are stacked along the $c$-axis in an alternating pattern.

Moreover, the rock salt-type $\mathrm{Ca}_{2} \mathrm{CoO}_{3}$ layer is reported to be responsible for the flexibility of the crystal structure due to oxygen vacancies, whereas the CdI2-type $\mathrm{CoO}_{2}$ layer accounting for maintaining the structural integrity ${ }^{[15]}$. The characteristic difference of the $b$ - axes subsystems make the crystal to present a misfit in such axes. The periodic difference between the rock salttype $\mathrm{Ca}_{2} \mathrm{CoO}_{3}$ and the $\mathrm{CdI}$-type $\mathrm{CoO}_{2}$ subsystems causes a significant displacement on each atom site, such differences entail an undulant arrangement for the $\mathrm{O}$ atom sites in the $\mathrm{CoO}_{2}$ subsystem with a less marked modulation in the Co sites. Additionally, significant shift modulation of the Co and $\mathrm{O}$ sites in the $\mathrm{Ca}_{2} \mathrm{CoO}_{3}$ subsystem has been reported ${ }^{[9]}$. In essence, the $\mathrm{CoO}_{2}$ subsystem is in a compressive-like state whereas the $\mathrm{Ca}_{2} \mathrm{CoO}_{3}$ subsystem remains in a tensile-like state due to their misfit in the $b$-axis. Moreover, the strains within the crystal cell could affect the overall behavior of the thermoelectric transport properties of the material ${ }^{[16]}$. One important downside of $\mathrm{Ca}_{3} \mathrm{Co}_{4} \mathrm{O}_{9}$ is the fact that half of the constituent elements are oxygen with evidently low atomic mass, leading to high lattice thermal conductivity, thus, making it a major challenge for improving the thermoelectric performance of the material ${ }^{[17]}$. 


\section{3 Enhancement of $\mathrm{Ca}_{3} \mathrm{Co}_{4} \mathrm{O}_{9}$ through ion doping and nano-inclusions}

Numerous studies have focused on ion doping via lattice substitution since this method remains one of the most promising approaches to enhance the thermoelectric capabilities in polycrystalline $\mathrm{Ca}_{3} \mathrm{Co}_{4} \mathrm{O}_{9}$, particularly, partial substitution of the $\mathrm{Ca}^{2+}$ site, whereas a minority have focused on the partial substitution of the $\mathrm{Co}^{3+} / \mathrm{Co}^{4+}$ site. Different materials have been reported to successfully improve the thermoelectric performance of $\mathrm{Ca}_{3} \mathrm{Co}_{4} \mathrm{O}_{9}$ ceramics. Enhancement of thermal conductivity and Seebeck coefficient values has been reported with Terbium ion doping in the $\mathrm{Ca}^{2+}$ site ${ }^{[18]}$. Mainly effects contributing to decrease the thermal conductivity values correspond to phonon scattering caused by the heavier $\mathrm{Tb}$ ions, at the same time the $\mathrm{Tb}$ doping causes a relative reduction in the concentration of $\mathrm{Co}^{4+}$ leading to an unusual increase in the Seebeck coefficient. Rare earth elements have been discussed to provide with such thermoelectric properties improvements for $\mathrm{Ca}_{3} \mathrm{Co}_{4} \mathrm{O}_{9}$ ceramics.

The partial substitution for Ca by rare earth elements has been proved as effective in improving thermoelectric properties. $\mathrm{Ca}_{3-\mathrm{x}} \mathrm{Ln}_{\mathrm{x}} \mathrm{Co}_{4} \mathrm{O}_{9}(\mathrm{Ln}=\mathrm{Dy}, \mathrm{Er}, \mathrm{Ho}$, and $\mathrm{Lu})$ ceramics were studied by Nong et al. ${ }^{[19]}$ All lanthanide-doped samples displayed a higher electrical resistivity, which is attributed to a decrease of $\mathrm{Co}^{4+}$ (hole carrier). More significantly, doped samples resulted in an astounding increase of Seebeck coefficient correlated with increasing Ln concentration, which is attributed to the $\mathrm{Ln}^{3+}$ substitution for $\mathrm{Ca}^{2+}$ (decreasing the $\mathrm{Co}^{4+}$ carrier concentration). Particularly, Lu-doped samples exhibited the best electrical power factor performance of $0.398 \mathrm{mWm}^{-1} \mathrm{~K}^{-2}$. Coupled with a low thermal conductivity due to the inclusion of the heavier $\mathrm{Lu}^{3+}$ ions, a ZT of 0.36 at $1073 \mathrm{~K}$ is attained for the $\mathrm{Ca}_{2.8} \mathrm{Lu}_{0.2} \mathrm{Co}_{4} \mathrm{O}_{9}$ ceramic ${ }^{[19]}$. Similarly, Klyndyuk et al. ${ }^{[20]}$ explored a higher doping level for $\mathrm{Ca}_{2 .} \mathrm{Ln}_{0.2} \mathrm{Co}_{4} \mathrm{O}_{9}$ ( $\mathrm{Ln}=\mathrm{La}, \mathrm{Nd}, \mathrm{Sm}$, Tb, Er), obtaining an extrapolated ZT of 0.4 at $1100 \mathrm{~K}$ for $\mathrm{Ca}_{2.8} \mathrm{Er}_{0.2} \mathrm{Co}_{4} \mathrm{O}_{9}$. Whereas, Pei et al. ${ }^{[21]}$ reported similar 
thermoelectric enhancement mechanisms for Er doping at a higher concentration. A figure of merit of 0.28 at $1073 \mathrm{~K}$ is achieved for $\mathrm{Ca}_{2.7} \mathrm{Er}_{0.3} \mathrm{Co}_{4} \mathrm{O}_{9}$. Chen et al. ${ }^{[22]}$ further studied the correlation between the electronic structure and the thermoelectric properties of $\mathrm{Ca}_{3-\mathrm{x}} \operatorname{Ln}_{\mathrm{x}} \mathrm{CO}_{4} \mathrm{O}_{9}$. They concluded that the smaller number of Co $3 d$ unoccupied states leads to, demonstrating the lesser amount of $\mathrm{Co}^{4+}$ concentration, a larger Seebeck coefficient ${ }^{[22]}$.

Nong et al., ${ }^{[23]}$ proposed an approach to improve the thermoelectric properties of the materials by introducing $\mathrm{Lu}^{3+}$ and metallic nanoinclusions. The Ag-rich regions and nanoinclusions showed to enhance both the electrical conductivity and Seebeck coefficient simultaneously. A maximum ZT value of 0.61 was achieved with $\mathrm{Ca}_{3} \mathrm{Ag}_{0.05} \mathrm{Lu}_{0.15} \mathrm{Co}_{4} \mathrm{O}_{9}$. Such value is achieved by a low thermal conductivity which they attributed to a combination of heavy doping of $\mathrm{Lu}$, nanoinclusion of $\mathrm{Ag}$, the grain size distribution, high angle boundaries, and randomly orientated grains which can lead to an increase in scattering of phonons. However, such low reported thermal conductivity values have been discussed as a significant overestimation of the $\mathrm{ZT}$ values due to speculation that the calculation was based on the out-of-plane thermal conductivity instead of the in-plane values ${ }^{[24]}$. Nonetheless, they provided an important approach to integrate heavily doped ceramic with embedded nanoinclusions within the grain interior. As show in Figure 2-2, starting from a state of randomly oriented grains to a more preferentially aligned crystals with embedded nanoinclusions is a potential method to increase the thermoelectric properties of $\mathrm{Ca}_{3} \mathrm{Co}_{4} \mathrm{O}_{9}$ ceramics. A very similar approach is reported by Butt et al., ${ }^{[13]}$ by adding $\mathrm{Cd}$ into the $\mathrm{Ca}_{3} \mathrm{Co}_{4} \mathrm{O}_{9}$, they were able to effectively incorporate $\mathrm{Cd}$ into the lattice substituting the $\mathrm{Ca}$ site to form single-phase polycrystalline $\mathrm{Ca}_{3} \mathrm{Co}_{4} \mathrm{O}_{9}$, and simultaneously generating $\mathrm{CdO}$ nanoinclusions, reaching a $\mathrm{ZT}$ of 0.35 at $1000 \mathrm{~K}$ for $\mathrm{Ca}_{2.9} \mathrm{Cd}_{0.1} \mathrm{Co}_{4} \mathrm{O}_{9}$. Where the $\mathrm{CdO}$ nanoinclusions serve to scatter the long wavelength phonons to reduce the lattice thermal conductivity. Simultaneously, Cd-doping 
benefits the suppression of the $\mathrm{Co}^{4+}$ concentration which enhanced the spin entropy and consequently rise the Seebeck coefficient. The decreased electrical resistivity was attributed to both a scattering of carriers which decreased the mobility and increased charge carrier generated in the $\mathrm{Ca}_{2} \mathrm{CoO}_{3}$ layer ${ }^{[13]}$.
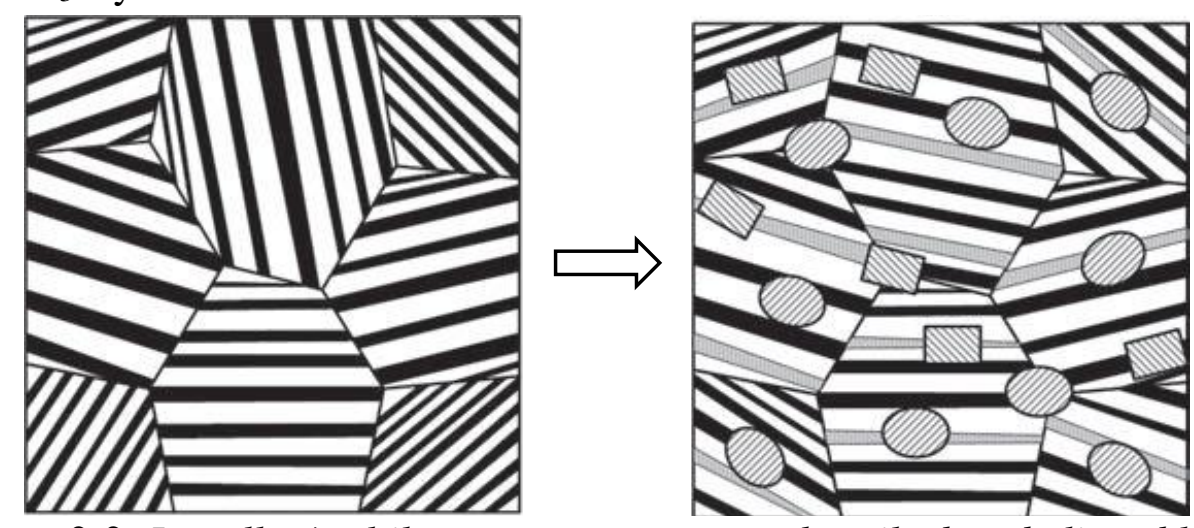

Figure 2-2: Lamellar/multilayer nanostructures to heavily doped aligned lamellar nanostructures with embedded nano-sized inclusions. ${ }^{[23]}$

Supplementary studies have explored the influence of Ag nanoparticles in the performance of the material. Even though these studies reported similar behavior as Nong et al. ${ }^{[23]}$, the overall ZT value for several studies were no even remotely close to such a high value. For instance Lin et al. ${ }^{[25]}$ reported a ZT value of 0.1 at $723 \mathrm{~K}$ with 2 vol\% addition of $\mathrm{Ag}$ nanoparticles. The stated values were also attained by a lower thermal conductivity and an increase of electrical conductivity simultaneously due to the increased phonon scattering, and increased grain orientation and carrier mobility. On the other hand, Park et al. ${ }^{[26]}$ described the structural and morphological properties of $\mathrm{Ca}_{3-\mathrm{x}} \mathrm{Ag}_{\mathrm{x}} \mathrm{Co}_{4} \mathrm{O}_{9+\delta}$. Enhancement of the thermoelectric properties of this doped material is possible to the existence of a mixed valence $(+3$ and +4$)$ of $\mathrm{Co}$ in the $\mathrm{CoO}_{2}$ layer and a mixed valence $(+2,+3$, and +4$)$ in the $\mathrm{CoO}$ of the $\mathrm{Ca}_{2} \mathrm{CoO}_{3}$ layer ${ }^{[26]}$. In addition, Zhang et al. ${ }^{[27]}$ presented and improvement of $70 \%$ in the electrical power factor for $\mathrm{Ca}_{2.7} \mathrm{Ag}_{0.3} \mathrm{Co}_{4} \mathrm{O}_{9}$ due to an optimized crystal orientation and shortcut effect giving way to increased activation energy leading to simultaneous increase in Seebeck and electrical conductivity. Kahraman et al. ${ }^{[28]}$ showed a 
outstandingly improvement of the electrical power factor for Ag addition. Reporting a raise in the samples densities, due to the formation of a liquid phase which improves sinterability, in addition to drastically better mechanical properties and decreases electrical resistivity. Furthermore, Wang et $\mathrm{al}^{[29]}$ conducted a comparison presenting, that as a matter of fact, is possible to use both approaches, combining substitution of $\mathrm{Ag}^{+}$for $\mathrm{Ca}^{2+}$ and non-stoichiometric addition of $\mathrm{Ag}$, to further boost the ZT to a 0.5 value at $1000 \mathrm{~K}^{[29]}$. In this case the ZT was achieved by a high electrical power factor, instead of a low thermal conductivity. The effect of Ag with different dual doping elements have been studied by several authors, the dual doping ceramics reported are $\left(\mathrm{Ca} 0 .{ }_{0} \mathrm{BiO}_{0.05}\right)_{3} \mathrm{Co}_{4} \mathrm{O}_{9} / \mathrm{Ag}^{[30]}, \quad \mathrm{Ag} /\left(\mathrm{Ca}_{0.975} \mathrm{La}_{0.025}\right)_{3} \mathrm{Co}_{4} \mathrm{O}_{9}{ }^{[31]}, \quad\left(\mathrm{Ca}_{0.9} \mathrm{Gd}_{0.1}\right)_{3} \mathrm{Co}_{4} \mathrm{O}_{9} / \mathrm{Ag}^{[32]}$, $\mathrm{Ag}_{\mathrm{x}} \mathrm{Y}_{\mathrm{y}} \mathrm{Ca}_{2.8} \mathrm{Co}_{4} \mathrm{O}_{9}{ }^{[33]}$, and $\mathrm{Ba}_{\mathrm{x}} \mathrm{Ag}_{\mathrm{y}} \mathrm{Ca}_{2.8} \mathrm{Co}_{4} \mathrm{O}_{9}{ }^{[34]}$. Although these dual doping allegedly improve the electrical properties of the materials, the thermal conductivity is not discussed, therefore lacking pertinent information to determine their overall performance of the thermoelectric figure of merit ZT.

In a similar approach, carbon nanotubes (CNT) have been reported to decrease thermal conductivity ${ }^{[17]}$. Integrating CNTs, with estimated size 80-160 nm, into $\mathrm{Ca}_{3} \mathrm{CO}_{4} \mathrm{O}_{9}$ ceramics lead to crystal defects at interfaces and increase the density of grain boundaries, giving rise to additional lattice scattering. Thermal conductivity $0.3 \mathrm{WK}^{1} \mathrm{~m}^{1}$ at room temperature is reported for $6 \mathrm{wt} . \%$ CNTs doped $\mathrm{Ca}_{3} \mathrm{Co}_{4} \mathrm{O}_{9}$. The nanocomposites made from $\mathrm{CNTs}$ embedded into $\mathrm{Ca}_{3} \mathrm{Co}_{4} \mathrm{O}_{9}$ exhibit highly porous structure. As a result, the low thermal conductivity is credited to increasing phonon scattering at nano-defects induced by the CNTs, porous structure, and the $\mathrm{Ca}_{3} \mathrm{Co}_{4} \mathrm{O}_{9} / \mathrm{CNTs}^{\prime}$ boundaries. Nonetheless, the increasing defects and porous structure cause the electrical resistivity to increase noticeably. Additionally, $\mathrm{Ca}_{3} \mathrm{Co}_{4} \mathrm{O}_{9} / \mathrm{ZrO}_{2}$ composites were studied by Gupta et al. ${ }^{[35]}$. The reason of studied the effect of $\mathrm{ZrO}_{2}$ was due to its low bulk thermal conductivity, high melting 
point and good chemical stability. The results showed no unwanted reaction occurred between $\mathrm{Ca}_{3} \mathrm{Co}_{4} \mathrm{O}_{9}$ and $\mathrm{ZrO}_{2}$ particles during the sintering process. $\mathrm{ZrO} 2$ particles were located at the grain boundaries of $\mathrm{Ca}_{3} \mathrm{Co}_{4} \mathrm{O}_{9}$, partly forming agglomerates and slight porosity in the material. Electrical resistivity showed remarkably lower values for all composite samples due to their larger grain size. However, all the composite materials also presents low Seebeck coefficient, therefore, achieving only a small increase in the power factor.

Furthermore, efforts to explore the effect on ion doping related to different doping elements segregated to the grain boundary has been reported as a promising approach to significantly increase the electrical power factor performance in thermoelectric ceramics. Previous work has demonstrated that $\mathrm{Ba}^{[36]}, \mathrm{Bi}^{[37]}$, and a co-doping of both $\mathrm{Bi}$ and $\mathrm{Ba}^{[38]}$ could be introduced into $\mathrm{Ca}_{3} \mathrm{Co}_{4} \mathrm{O}_{9}$ ceramics using either substitution, non-stoichiometric addition, and a combination of both, respectively, to improve the texture of the crystal, and enhance the Seebeck coefficient and electrical conductivity at the same time while decreasing the thermal conductivity, thus achieving a greater ZT value. Non-stoichiometric addition of $\mathrm{Ba}^{2+}$ segregates only to the grain boundary, thus, resulting beneficial the non-stoichiometric approach rather than substitution, since it is not incorporated into the lattice but just acting simply as an impurity. Moreover, substitution of $\mathrm{Bi}^{3+}$ is reported to be incorporated into both the lattice and grain boundary. However, for the co-doping of $\mathrm{Ba}^{2+}$ and $\mathrm{Bi}^{3+}$ selective segregation is reported. The authors identified the strain effect as a driving force for segregation, hence the smaller $\mathrm{Bi}^{3+}$ is displaced to the grain interior by the bigger over-sized $\mathrm{Ba}^{2+}$ atoms. Furthermore, oxidation states are different for $\mathrm{Bi}^{3+}$ and $\mathrm{Ba}^{2+}$, still both elements segregates at the grain boundary when introduced into the $\mathrm{Ca}_{3} \mathrm{Co}_{4} \mathrm{O}_{9}$ as the only dopant, suggesting that segregation is ionic size controlled rather than valence state controlled. The grain boundary segregation of the dopants is reported to serve as a filter where low energy carriers are 
filtered and not contributing to the conduction, leading to a reduced carrier concentration. ${ }^{[36-38]}$ As a result, the selective scatter of low-energy carriers drastically improved the Seebeck coefficient. Whereas improvement of the electrical conductivity is attributed to the crystal texture and alignment of the grains which lead to a higher carrier mobility. Phonon scattering effects are mainly attributed to reduce the thermal conductivity in this grain boundary segregation approach.

The effect of dual doping of $\mathrm{Ce}$ and $\mathrm{Ni}$ to form $\mathrm{Ca}_{3-\mathrm{x}} \mathrm{Ce}_{\mathrm{x}} \mathrm{Co}_{4-\mathrm{y}} \mathrm{Ni}_{\mathrm{y}} \mathrm{O}_{9+\delta}$ materials was investigated by Tang et al. ${ }^{[39]}$ at lower temperatures. Samples were prepared by the sol-gel method to form the stoichiometric chemistry. Their XRD results showed small shift toward higher $2 \theta$ values due to the smaller ionic radii of $\mathrm{Ni}^{2+} / \mathrm{Ni}^{3+}$ compared with that of $\mathrm{Co}^{2+}$ and diffraction peaks shift toward lower angle as $\mathrm{Ce}$ content increased, because $\mathrm{Ce}^{3+}$ ions have a larger ionic radius than the replaced $\mathrm{Ca}^{2+}$ ions. Thus, they reported that $\mathrm{Ce}$ and $\mathrm{Ni}$ were successfully doped into the $\mathrm{Ca}_{3} \mathrm{Co}_{4} \mathrm{O}_{9}$ lattice. The effect of substitution of $\mathrm{Ce}^{3+}$ for $\mathrm{Ca}^{2+}$ was found to reduce carrier concentration, thus, reducing the electrical resistivity. Additionally adding $\mathrm{Ni}$ and the heavy element $\mathrm{Ce}$ act as scattering points, by this means decreasing phonon contribution and decreasing $\kappa_{p h}{ }^{[39]}$. The incorporation of $\mathrm{Lu}$ instead of $\mathrm{Ce}$ for dual doping $\mathrm{Ca}_{3-\mathrm{x}} \mathrm{Lu}_{\mathrm{x}} \mathrm{CO}_{4-\mathrm{y}} \mathrm{Ni}_{\mathrm{y}} \mathrm{O}_{9}$ is reported to improve the thermoelectric properties by improving the Seebeck coefficient attributed to a increase of the spin entropy, and by increasing phonon scattering, thus achieving a decrease of thermal conductivity. Nonetheless, using $\mathrm{Lu}$ as the second of dual dopants instead of Ce improve the performance to a lesser extent (a reduction of $15 \%$ ZT compared to Ce-Ni dual doping) ${ }^{[40]}$. The aforementioned studies were made after Park et al. ${ }^{[41]}$ reported a remarkably increase on the electrical power factor for $\mathrm{Ni}$ doping into the Co site of $\mathrm{Ca}_{3} \mathrm{Co}_{4} \mathrm{O}_{9}$.

The influence on thermoelectric performance of $\mathrm{Ca}_{3} \mathrm{Co}_{4} \mathrm{O}_{9}$ by dual doping of $\mathrm{Y}$ and $\mathrm{Fe}$ has been reported with a ZT value of 0.2 at $1073 \mathrm{~K}^{[42]}$. Single phase $\mathrm{Ca}_{3} \mathrm{Co}_{4} \mathrm{O}_{9}$ was found for all $\mathrm{Fe}$ - 
doped and Fe/Y co-doped samples with crystal texture due to doping and SPS. They described the effect of Fe substitution is believed to be the origin of the lower resistivity by increasing hole carriers. This was assumed due to increasing $\mathrm{O}^{2-}$ unoccupied states and oxygen content upon $\mathrm{Fe}$ doping. They also found that trivalent $\mathrm{Y}^{3+}$ substitutions for divalent $\mathrm{Ca}^{2+}$ ions may reduce the $\mathrm{Co}^{4+}$ (hole) concentration. Thus the resistivity of $\mathrm{Y}$-doped $\mathrm{Ca}_{3} \mathrm{Co}_{4} \mathrm{O}_{9}$ was higher than those in the undoped samples. Moreover, reduction of $\kappa_{\mathrm{ph}}$. was be attributed to the heavy $\mathrm{Y}^{3+}$ substitution for $\mathrm{Ca}^{2+[42]}$. Similarly, Butt et al. ${ }^{[43]}$ obtained a higher ZT value of 0.32 at $1000 \mathrm{~K}$ on dual doping of Fe into $\mathrm{Ca}_{3} \mathrm{Co}_{4} \mathrm{O}_{9}$ but with $\mathrm{La}$ as a second dopant. A small shift of the $2 \theta$ angles is reported for all peaks due to bigger ionic radii of the dopants indicating that $\mathrm{Fe}$ and $\mathrm{La}$ have been incorporated into the lattice. The substitution of a lower valance state of $\mathrm{Fe}$ at the $\mathrm{Co}$ site in $\mathrm{Ca}_{2} \mathrm{CoO}_{3}$ layers increased the carrier concentration, while the partial substitution of $\mathrm{La}^{3+}$ at the $\mathrm{Ca}^{2+}$ site had the opposite effect. The decrease in carrier concentration resulted in an increase of the Seebeck coefficient. Additionally, $\mathrm{La}^{3+}$ substituted for $\mathrm{Ca}^{2+}$ induced lattice distortions due to their bigger ionic radii that increase phonon scattering and suppress $\kappa_{\mathrm{ph}}{ }^{[43]}$.

Huang et al. ${ }^{[44]}$, explored the influence of $\mathrm{Ba}$ and $\mathrm{Pr}$ incorporated into $\mathrm{Ca}_{3} \mathrm{Co}_{4} \mathrm{O}_{9}$, displaying a ZT of 0.31 at $973 \mathrm{~K}$ for the best concentration, surpassing the ZT of 0.24 at $973 \mathrm{~K}$ obtained for Pr doping alone ${ }^{[45]}$. It was found that XRD diffraction peaks exhibited a slight $2 \theta$ shift and that peaks of $(00 l)$ planes are stronger than other peaks, pointing out the preferred grain orientation. Doping $\mathrm{Ba}$ and $\mathrm{Pr}$ into the $\mathrm{Ca}_{3} \mathrm{Co}_{4} \mathrm{O}_{9}$ resulted in controlled grain orientations by varying the dopant concentration. This led to less carrier scattering between grains and inter-sub layers, subsequently decreasing the electrical resistivity. Also, it was found the dual doped material with higher $\mathrm{Pr}$ concentration have irregular grain size distribution. This was favorable for enhancing phonon scattering due to the enlarged crystal boundaries, thus, leading to a decrease of $\kappa_{\mathrm{ph}}$ by the reduction 
of the phonon mean free path, achieving a $\kappa=1.5 \mathrm{Wm}^{-1} \mathrm{~K}^{-1[44]}$. Supplementary information in the dual doping of $\mathrm{Ba}$ and $\mathrm{Pr}$ into $\mathrm{Ca}_{3} \mathrm{Co}_{4} \mathrm{O}_{9}$ is given by Liu et al. ${ }^{[46]}$. In this study authors discussed sol-gel and solid state reaction as a precursor powders, concluding that sol-gel makes for the best precursor. In addition, it was reported that the electrical performance could also be further improved by modulating material particle orientation modification and attaining a uniform particle size $^{[46]}$. Nonetheless, a higher ZT for Ba alone is reported elsewhere ${ }^{[36,47]}$.

Composite materials made of $\mathrm{Ca}_{3} \mathrm{Co}_{4} \mathrm{O}_{9}$ and $\mathrm{La}_{0.8} \mathrm{Sr}_{0.2} \mathrm{CoO}_{3}$ (LSCO) were reported to achieve a ZT of 0.41 with a thermal conductivity around $1.15 \mathrm{Wm}^{-1} \mathrm{~K}^{-1}{ }^{[48]}$. Results exhibited that the constituents retained their individual crystalline and morphological characteristics. The nanosized LSCO particles are responsible for scattering of long-wavelength phonons at the grain boundaries, reducing the thermal conductivity. Is reported as well that LSCO particles occupied in the voids of $\mathrm{Ca}_{3} \mathrm{Co}_{4} \mathrm{O}_{9}$ grains act as electrical connection, which increase the electrical conductivity. Additionally, enhancement of the Seebeck effect by a compressive strain induced into the $\mathrm{Ca}_{3} \mathrm{Co}_{4} \mathrm{O}_{9}$ grains by the LSCO nanoparticles is identified in the study of the composite ${ }^{[48]}$.

Previous studies on $\mathrm{Gd}$ doping on $\mathrm{Ca}_{3} \mathrm{Co}_{4} \mathrm{O}_{9}$ (Ca-site substitution) ceramics indicated a maximum figure of merit $\mathrm{ZT}$ of 0.24 at $973 \mathrm{~K}$ obtained for $\mathrm{Ca}_{2.7} \mathrm{Gd}_{0.3} \mathrm{Co}_{4} \mathrm{O}_{9}{ }^{[49]}$. Their $\mathrm{XRD}$ patterns showed single phase compounds and that $\mathrm{Gd}$ was doped into the lattice of $\mathrm{Ca}_{3} \mathrm{Co}_{4} \mathrm{O}_{9}$. Alignment in the crystal grains is improved with the addition of $\mathrm{Gd}$, however the grain interior displayed increased defects. The electrical conductivity is affected mainly due to decrease the carrier concentration. Whereas the Seebeck coefficient is improved due to the reduction of carrier concentration, and the change of spin state of $\mathrm{Co}$ ion in $\mathrm{CoO}_{2}$ layers. Besides, the thermal conductivity is reduced by the impurity scattering created by the grain interior defects ${ }^{[49]}$. A similar behavior in the thermoelectric performance is reported for Y-doped samples with the 
$\mathrm{Ca}_{2.7} \mathrm{Y}_{0.3} \mathrm{Co}_{4} \mathrm{O}_{9}$ concentration achieving a maximum figure of merit $\mathrm{ZT}$ of 0.22 at $973 \mathrm{~K}^{[50]}$. Ydoped samples present single phase ceramics with improved Seebeck values but a deteriorated electrical conductivity due to a reduction of carrier concentration. Y-doped samples also exhibit a lower thermal conductivity due to impurity scattering within the crystals. Furthermore, the addition of a second element as a dual doping has been reported to improve the maximum ZT value to 0.26 at $973 \mathrm{~K}$ for $\mathrm{Gd}$ and $\mathrm{Y}$. The improvements mechanism are reported to be the same for the dual doping $\mathrm{Ca}_{3} \mathrm{Co}_{4} \mathrm{O}_{9}$ ceramics ${ }^{[51]}$.

Ti-doping has showed a promising thermoelectric figure of merit ZT value of 0.3 at $1000 \mathrm{~K}$ for $\mathrm{Ca}_{3} \mathrm{Co}_{3.7} \mathrm{Ti}_{0.3} \mathrm{O}_{9}$ samples ${ }^{[52]}$. Single phase ceramics with a slight shift in the $2 \theta$ angles due to different ionic radii is reported for the doped samples. Crystal texture and alignment of the crystal grains is promoted with the addition of the dopant. Ti-doped samples show a thermal conductivity reduction due to the incorporation of $\mathrm{Ti}$ ions into the $\mathrm{Ca}_{3} \mathrm{Co}_{4} \mathrm{O}_{9}$ lattice, such addition induce disorder and structural distortion, which can strongly scatter phonons. Compared to baseline samples the doped samples exhibited a greater electrical resistivity due to a rise in the carrier concentration, whose value is also attributed to the enhancement of the Seebeck coefficient. Nonetheless, the remarkably increase in Sebbeck for $\mathrm{Ca}_{3} \mathrm{Co}_{3.7} \mathrm{Ti}_{0.3} \mathrm{O}_{9}$ is additionally attributed to the substitution of $\mathrm{Ti}$ for $\mathrm{Co}$ in both the $\mathrm{Ca}_{2} \mathrm{CoO}_{3}$ and $\mathrm{CoO}_{2}$ layers ${ }^{[52]}$. However Hu et al. research have shown there is no change in the Co valence state, the Co-ion spin state, or the overall charge carrier concentration in the $\mathrm{CoO}_{2}$-layers compared to pristine $\mathrm{Ca}_{3} \mathrm{Co}_{4} \mathrm{O}_{9}{ }^{[53]}$. In addition, Torres et al. ${ }^{[54]}$ reported that Ti-doping does not significantly alter the Seebeck coefficient values, even so their doped samples present an improved electrical power factor due to a decrease in the electrical resistivity. Thus, presenting contradictory information on the thermoelectric performance of this dopant. Dual doping of $\mathrm{Ti}$ and $\mathrm{Zn}$ has also been discussed as a possible way to enhance the 
thermoelectric properties of $\mathrm{Ca}_{3} \mathrm{Co}_{4} \mathrm{O}_{9}$, although showing a less promising ZT mostly a result of high electrical resistivity values ${ }^{[55]}$.

Some studies have explored the influence of $\mathrm{Yb}$ substitution on the thermoelectric properties of the $\mathrm{Ca}_{3} \mathrm{Co}_{4} \mathrm{O}_{9}$ in the low temperature region ${ }^{[56,57]}$. An overall low improvement is obtained on the ceramic materials due to deterioration of the electrical power factor. The extra electrons introduced by substitution of $\mathrm{Yb}^{3+}$ for $\mathrm{Ca}^{2+}$ in the $\mathrm{Ca}_{3} \mathrm{Co}_{4} \mathrm{O}_{9}$ system resulted in a hole concentration reduction which lead to a high electrical resistivity. More importantly, the impurities and defects created by the Yb-substitution induce scattering of phonons leading to a shorten mean free path of the phonons and causes a strong decrease in thermal conductivity ${ }^{[57]}$. In this assessment, Song et al. ${ }^{[58]}$ have reported an effective approach to improve the electrical transport properties and Seebeck coefficient of $\mathrm{Ca}_{3} \mathrm{Co}_{4} \mathrm{O}_{9}$ ceramics through $\mathrm{Yb}$ doping at high temperature to a power factor of $553 \mathrm{mWm}^{-1} \mathrm{~K}^{-2}$ for $\mathrm{Ca}_{2 .} \mathrm{Yb}_{0.1} \mathrm{Co}_{4} \mathrm{O}_{9}$ pressed at $0.5 \mathrm{GPa}$. Improvements come from controlling the nanostructure by cold pressing, whereas the $\mathrm{Yb}$ doping affect both the carrier mobility and carrier concentration ${ }^{[58]}$.

The framework information of doping polycrystalline $\mathrm{Ca}_{3} \mathrm{Co}_{4} \mathrm{O}_{9}$ gives a general view of the great impact of ion doping on the properties of the $\mathrm{Ca}_{3} \mathrm{Co}_{4} \mathrm{O}_{9}$, for this reason, a broad range of doping and dual doping elements have been reported to understand the different mechanisms associated with different dopants. Currently, to be able to promote an increase on the thermoelectric figure of merit that surpass a threshold of ZT value of 0.4 is still a difficult task. For instance, some transition metals increase the $\mathrm{ZT}$ to a mere 0.12 at $973 \mathrm{~K}^{[59]}$, others such as the

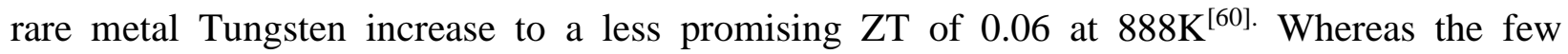
surpassing that threshold as aforementioned for elements such as $\mathrm{Tb}^{[18]}, \mathrm{Ag}^{[23]}, \mathrm{Ba}^{[36]}, \mathrm{Bi}^{[37]}, \mathrm{Bi}^{-}$ $\mathrm{Ba}^{[38]}$, and others ${ }^{[24]}$, make for the minority of exceptions with a high $\mathrm{ZT}$ for polycrystalline 
$\mathrm{Ca}_{3} \mathrm{Co}_{4} \mathrm{O}_{9}$. Yet, developing outstanding element doping parameters is still a great opportunity to expand and improve the performance with optimized synthesis conditions.

\section{4 Enhancement of $\mathrm{Ca}_{3} \mathrm{Co}_{4} \mathrm{O}_{9}$ through modification of the processing techniques}

$\mathrm{Ca}_{3} \mathrm{Co}_{4} \mathrm{O}_{9}$ ceramics carriage a remarkable thermoelectric performance with a figure of merit ZT of 0.87 at $973 \mathrm{~K}$ for the single crystal material ${ }^{[61]}$. Nonetheless, the single crystal alternative is not appropriate candidate for practical applications, mainly due to excessive production cost and complexity of processing materials as single crystals in abundant quantities. Consequently, polycrystalline $\mathrm{Ca}_{3} \mathrm{Co}_{4} \mathrm{O}_{9}$ ceramics remain as the best scheme to be use as a thermoelectric material to develop solid-state energy conversion systems for a future commercial purpose. Although, polycrystalline $\mathrm{Ca}_{3} \mathrm{Co}_{4} \mathrm{O}_{9}$ materials are inexpensive and easier to process, they have the drawback of not possessing the same remarkable thermoelectric performance. Hence, the influence of different processing techniques has been a research topic to find optimal parameters of traditional and complex techniques in order to improve the thermoelectric characteristic of polycrystalline $\mathrm{Ca}_{3} \mathrm{Co}_{4} \mathrm{O}_{9}$.

Synthesis process of calcium cobaltite is one key aspect to optimize in order to obtain desirable thermoelectric properties of the material. Although, the influence of the thermoelectric properties with different set of parameters by solid state reaction has been reported as an approach to obtain $\mathrm{Ca}_{3} \mathrm{Co}_{4} \mathrm{O}_{9}$ powders ${ }^{[62]}$, sol-gel chemical route is reported to significantly reduce the secondary phase content in $\mathrm{Ca}_{3} \mathrm{Co}_{4} \mathrm{O}_{9}$ compared to conventional solid state reaction ${ }^{[63]}$, due to the ability of the sol-gel method to produce more homogeneous powders (although, single phase with no other impurity phase with the solid state reaction method has been reported ${ }^{[64]}$ ). Secondary phases such as $\mathrm{Co}_{3} \mathrm{O}_{4}$ has been reported to decrease the performance of the material ${ }^{[65]}$. 

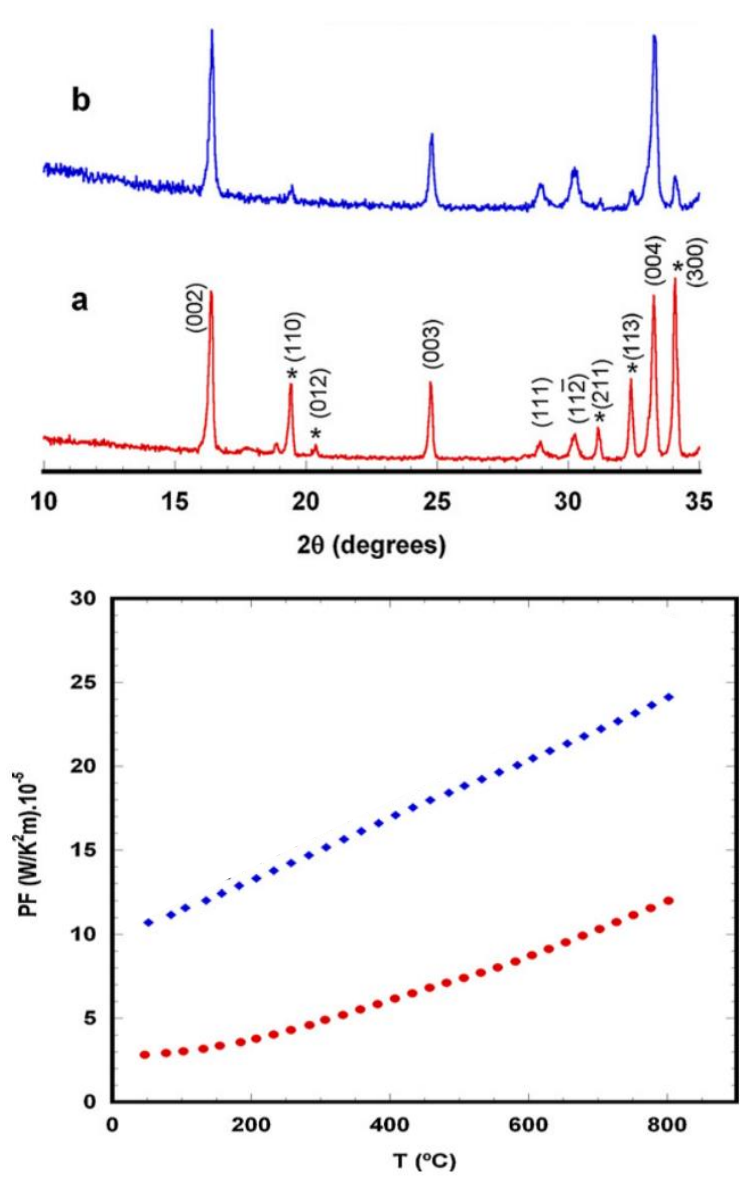
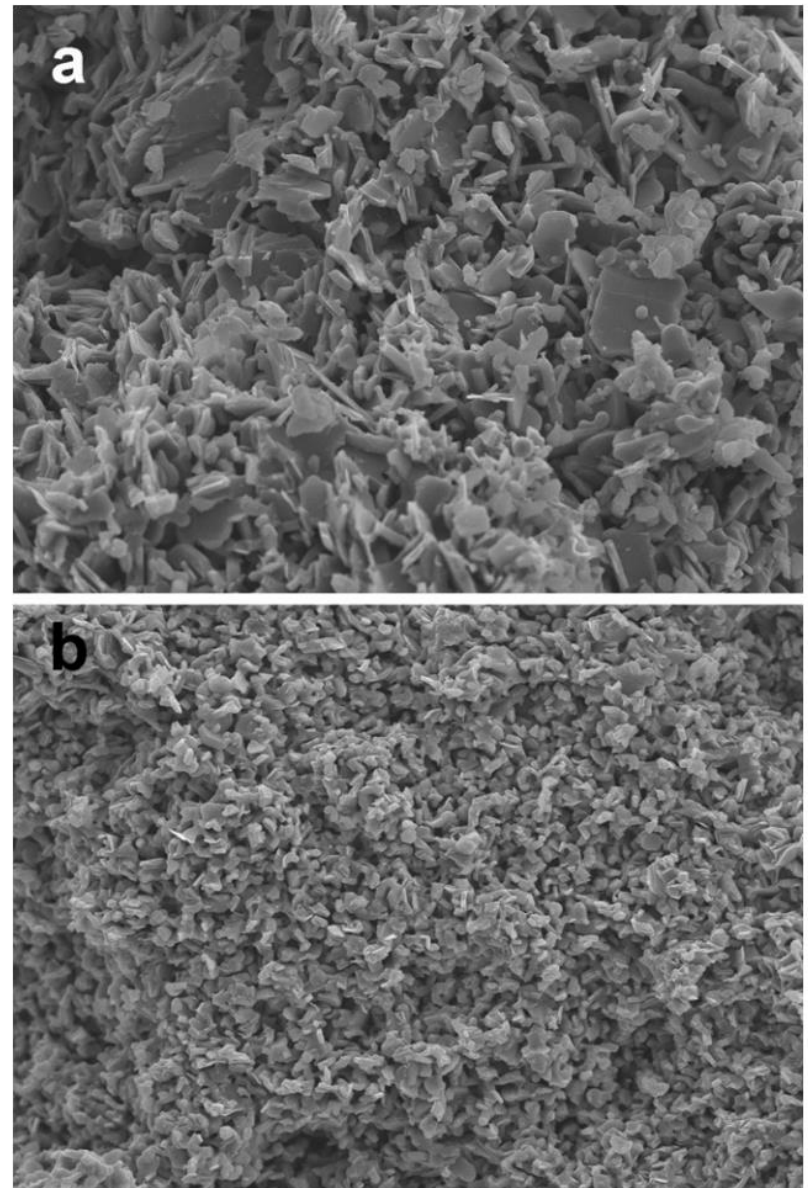

Figure 2-3: Left top: $X R D$ plots of the $\mathrm{Ca}_{3} \mathrm{Co}_{4} \mathrm{O}_{9}$ sintered specimens obtained for: (a) Solid state, (b) sol-gel. Crystallographic planes are indicated for the $\mathrm{Ca}_{3} \mathrm{Co}_{4} \mathrm{O}_{9}$ phase and $\mathrm{Ca}_{3} \mathrm{Co}_{2} \mathrm{O}_{6}$ (identified by a

*).Left bottom: Temperature dependence of the power factor for $\mathrm{Ca}_{3} \mathrm{Co}_{4} \mathrm{O}_{9}$, for: (•) Solid state; ( $\bullet$ solgel. Right: SEM obtained on transversal fractured $\mathrm{Ca}_{3} \mathrm{Co}_{4} \mathrm{O}_{9}$ samples prepared by solid-state (a), and sol-gel (b) ${ }^{[63]}$

Furthermore, Sotelo et al, discussed in detail the synthesis method for solid-state reaction and the sol-gel methods. The main improvement utilizing sol gel synthesis claimed was reduction on the electrical resistivity due to the remarkably reduction of secondary phases and smaller homogeneous ceramic powders which leaded to higher electrical power factor values than the reported for the solid state method. Additionally, sol-gel compromise very simple and reproducible processes $^{[63]}$. Moreover, auto-combustion of the sol-gel is reported to inherit the same characteristic of controlled stoichiometry of the $\mathrm{Ca}_{3} \mathrm{Co}_{4} \mathrm{O}_{9}$ with the added benefit of providing a faster method to synthesize the material ${ }^{[66]}$. 
Although nanostructuring has been proposed as an effective strategy for improving better performance in $\mathrm{Ca}_{3} \mathrm{Co}_{4} \mathrm{O}_{9}$ polycrystalline attaining nanometric grain size can be challenging. In order to get smaller and a more uniform grain size Soffientini et al. ${ }^{[67]}$ explored the effect of solgel synthesis material with different ball milling parameters. They concluded that materials presenting very small grain size and low crystallinity present a figure of merit that is generally inferior than the one shown by materials presenting sub-micrometric grain size ${ }^{[67]}$, therefore, selecting an appropriate set of parameters for the ball milling stage is essential to optimize the material process.

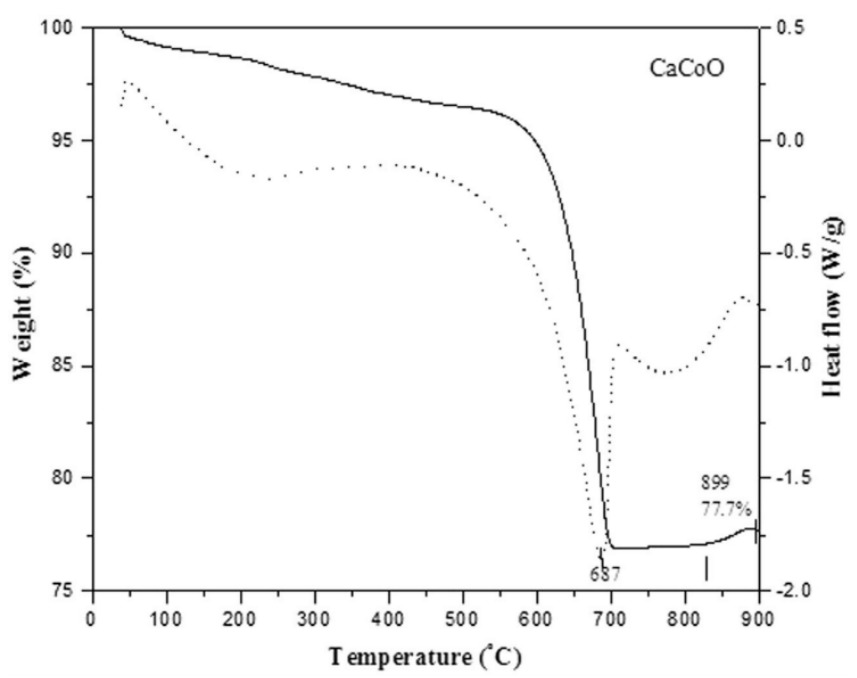

Figure 2-4: DSC/TGA curve of $\mathrm{Ca}_{3} \mathrm{Co}_{4} \mathrm{O}_{9 .}{ }^{[68]}$

Equally important, is the calcination and sintering temperatures and reaction conditions. X-ray diffraction patterns showed formation of secondary phases for longer calcination duration. Radhika et al. ${ }^{[68]}$, showed that formation of single-phase of $\mathrm{Ca}_{3} \mathrm{Co}_{4} \mathrm{O}_{9}$ takes place at around 700800C from the solid state reaction method, as shown by DSC/TGA curve of $\mathrm{Ca}_{3} \mathrm{Co}_{4} \mathrm{O}_{9}$. Their study revealed that the calcination of the material have a great influence in the density and nonstoichiometry of the material. Likewise, the carrier concentration and mobility was correlated with the calcination time ${ }^{[68]}$, and therefore have a direct influence in the thermoelectric performance of the material. Chen et al. ${ }^{[69]}$, reported the calcination temperature effect for $\mathrm{Ca}_{3} \mathrm{Co}_{4} \mathrm{O}_{9}$ bulk materials 
prepared by sol-gel method. They concluded the size of the nano-crystals could be controlled by varying the calcination temperature. The optimized temperature was found to promote large crystal growth and texture development during sintering, achieving the best $\mathrm{ZT}$ value with powders sintered at $673 \mathrm{~K}^{[69]}$. In addition, "heavy-calcination" and "moderate-grinding" steps before pelletizing showed a better crystal texture with plate-like crystal grains stacked up in layers, and large grains powders played a favorable role improving the orientation ${ }^{[62]}$. Notably, Karppinen et al. ${ }^{[70]}$ concluded that for samples synthesized in air or a more reducing atmosphere could produce considerable amounts of oxygen vacancies in $\mathrm{Ca}_{3} \mathrm{CO}_{4} \mathrm{O}_{9}$ influencing the thermoelectric performance of the material ${ }^{[70]}$, thus, adopting an oxygen environment during both the calcination and sintering stages could be beneficial to get improved thermoelectric properties.

It has been reported that using spark plasma (SPS) as a sintering method provide an approach to obtain highly dense materials compared to the theoretical density, relative density as high as $98 \%^{[71-73]}$. SPS facilitated development of a uniform grain morphology with an improved grain alignment in comparison to conventional sintering ${ }^{[71]}$. Furthermore, grain alignment during the SPS process yield a better electrical performance due to the layered structure and high anisotropy properties in the calcium cobaltite material compared with the conventional sintering given the porosity in the sample, which significantly reduces the electrical conductivity of the ceramics ${ }^{[74]}$. Samples synthesized by sol-gel followed by SPS are reported to achieve better thermoelectric performance than samples synthesized by solid state reaction followed by SPS ${ }^{[73,75]}$, reinforcing sol-gel as the best synthesis process. Additionally, the mechanical properties are also correlated with the microstructure and the crystal texture of the material. SPS and hot pressing offer better mechanical properties for $\mathrm{Ca}_{3} \mathrm{Co}_{4} \mathrm{O}_{9}$ ceramics ${ }^{[76]}$. 

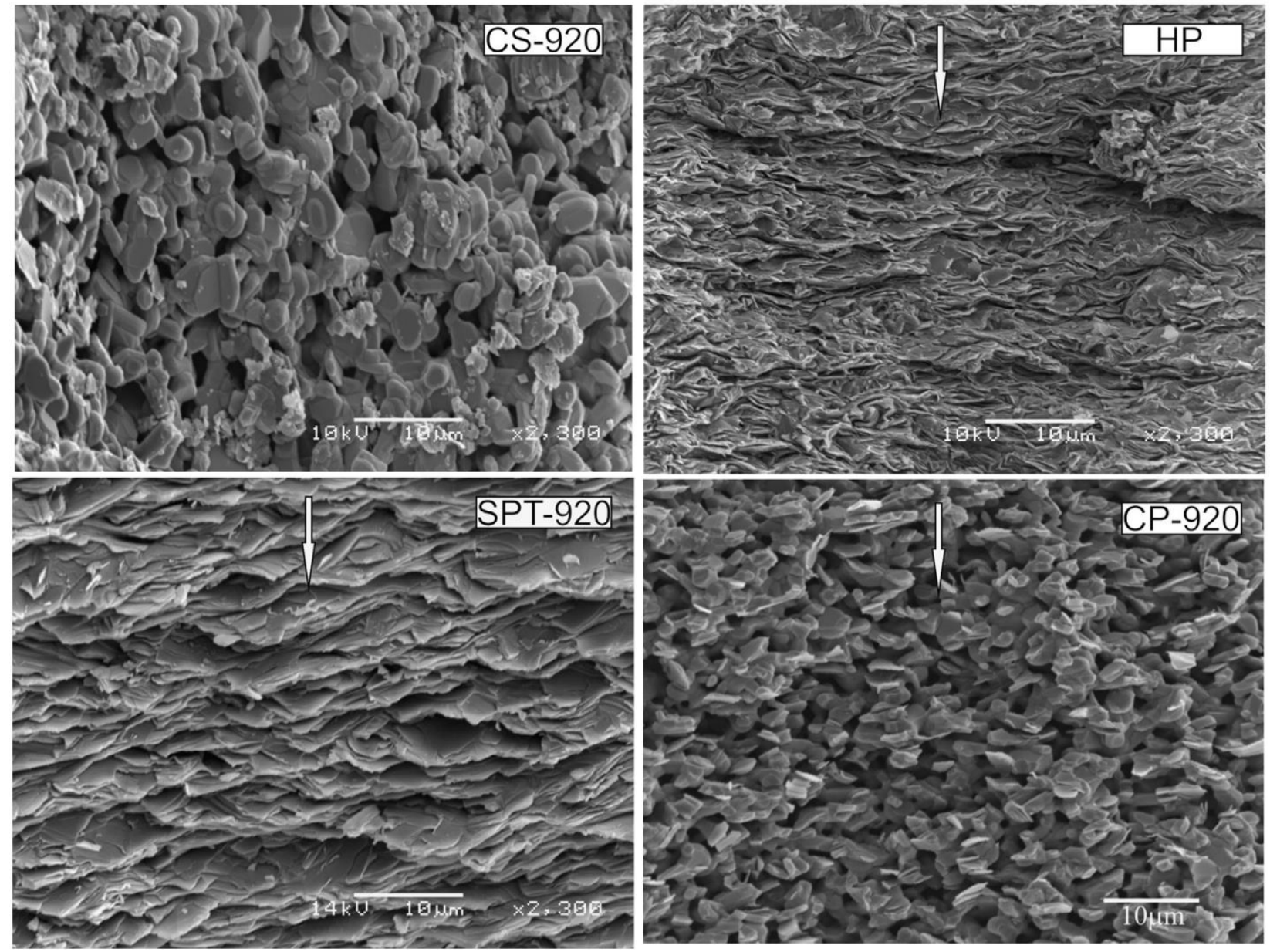

Figure 2-5: Comparison of the SEM images of the fractured surfaces of the $\mathrm{Ca}_{3} \mathrm{Co}_{4} \mathrm{O}_{9}$ samples prepared by classic sintering at $920^{\circ} \mathrm{C}(\mathrm{CS}-920)$, hot pressing (HP), SPS sintered at $920^{\circ} \mathrm{C}(\mathrm{SPT}-920)$, and cold pressed sample sintered at $920^{\circ} \mathrm{C}(\mathrm{CP}-920)$. The arrow indicates the stress direction. ${ }^{[64]}$

Furthermore, Presečnik et al. ${ }^{[64]}$ explored the influence of classic sintering (lower pressure than cold pressing), hot pressing, SPS, and cold pressing on the thermoelectric properties of $\mathrm{Ca}_{3} \mathrm{Co}_{4} \mathrm{O}_{9}$ ceramics synthesized by solid state reaction. They determined that a compromise between the density and the degree of texturing gives the best thermoelectric performance characteristics. For instance, SPS samples displayed perfectly aligned grains in the direction perpendicular to the applied stress and hence almost perfect texturing and high density resulting in a higher electrical conductivity but also a high thermal conductivity. Hot press samples also showed preferential alignment and a relative density of $94 \%$ having good electrical properties but then again affected thermal properties as well. Importantly, samples with a smaller grain size produced energy barriers 
at the grain boundaries, which influences a decrease of the charge carriers, improving the Seebeck coefficient, and also improving the thermal conductivity due to high porosity in the material. Consequently, conventional pressing presented the best thermoelectric properties due to the high porosity, slightly textured, and small plate-like grains, which resulted in figure of merit ZT of $0.31^{[64]}$.

Highly porous $\mathrm{Ca}_{3} \mathrm{Co}_{4} \mathrm{O}_{9}$ ceramics fabricated by sol-gel method and conventional pressing and sintering methods are reported to significantly decrease the thermal conductivity of the material down to $0.63 \mathrm{Wm}^{-1} \mathrm{~K}^{-1[77]}$ which is one of the lowest reported thermal conductivity for pure $\mathrm{Ca}_{3} \mathrm{Co}_{4} \mathrm{O}_{9}$, they also reported a record high figure of merit $\mathrm{ZT}$ of 0.4 for conventional, non-doped $\mathrm{Ca}_{3} \mathrm{Co}_{4} \mathrm{O}_{9}{ }^{[77]}$. The reported samples showed a low relative density of $67 \%$ compared to the single crystal, which could have a big influence on the reported low thermal conductivity. The main effect of porous materials is the suppression of thermal conductivity due to phonon boundary scattering at the increased number of interfaces ${ }^{[77,78]}$. Previous studies showed that nanostructuring and porosity do not appear to significantly affect the Seebeck coefficient. Conversely, the electrical resistivity an increase in the resistivity was apparent, leading to lower power factors for the nanostructuring and more porous samples ${ }^{[78]}$.

A peculiar study introducing wood particles in the $\mathrm{Ca}_{3} \mathrm{Co}_{4} \mathrm{O}_{9}$ showed a possible method to induce porosity in the material to obtain ZT of 0.2 and a $\kappa$ of $1.2 \mathrm{Wm}^{-1} \mathrm{~K}^{-1}$ at $800 \mathrm{~K}^{[79]}$. The results showed crystal structure remained unchanged with just a small increase in the ratio of $\mathrm{Co}^{3+} / \mathrm{Co}^{4+}$, such increase served to enhance the Seebeck coefficient of the porous samples due to a reduction of carrier concentration. The decrease in carrier concentration is attributed to the local oxygen deficient environment at the time of wood burn-off process. Nonetheless the main contribution of the porosity is a reduction in the thermal conductivity due to increased phonon scattering ${ }^{[79]}$. 


\section{5 Summary}

To recapitulate, most industrial processes suffer from a significant loss of energy in the form of waste heat. This energy could be reutilized and turned into an economic benefit by improving fuel efficiency. Thermoelectric materials are capable of directly convert waste heat into electricity. Furthermore, TE oxides have shown a promising thermoelectric performance at high temperature, particularly, $\mathrm{Ca}_{3} \mathrm{Co}_{4} \mathrm{O}_{9}$ ceramic is reported to be a good candidate as a p-type material due to the tunable layered structure. Nonetheless, the polycrystalline material only performs at $30-50 \%$ compared to the single crystal. To achieve a high performance TE material the electrical conductivity and Seebeck coefficient need to be increased and simultaneously decrease the thermal conductivity.

In this section, the different strategies to optimize the $\mathrm{Ca}_{3} \mathrm{Co}_{4} \mathrm{O}_{9}$ were reviewed. Nanoinclusions have been discussed to decrease the thermal conductivity by creating phono scattering events but provide little to none improvement on the Seebeck or electrical conductivity. On the other hand, ion doping has been showed to be crucial in the improvement of $\mathrm{Ca}_{3} \mathrm{Co}_{4} \mathrm{O}_{9}$. Doping with rare-earth elements is reported to improve the electrical and thermal transport properties of the polycrystalline $\mathrm{Ca}_{3} \mathrm{Co}_{4} \mathrm{O}_{9}$. Additionally, oversized atom doping ( $\mathrm{Bi}$, $\mathrm{Ba}$ ) has been reported to segregate at grain boundary which improves dramatically the TE performance. Substituting the host ions in $\mathrm{Ca}_{3} \mathrm{Co}_{4} \mathrm{O}_{9}$ by heavier atoms is reported to provide phonon scattering events which reduce the thermal conductivity. Modifying the synthesis processing is reported to be of big influence in the TE properties. Although complex synthesis techniques show good performance, high performance could also be achieved by tuning the sintering and calcination temperature and environment and with the use of conventional sol-gel and pressing methods. 


\section{References}

1. U.S., D.o.E., Quadrennial technology review: An assessment of energy technologies and research Opportunities 2015. p. 1-505.

2. Fergus, J.W., Oxide materials for high temperature thermoelectric energy conversion. Journal of the European Ceramic Society, 2012. 32(3): p. 525-540.

3. Woermann, E. and A. Muan, Phase equilibria in the system CaO-cobalt oxide in air. Journal of Inorganic and Nuclear Chemistry, 1970. 32(5): p. 1455-1459.

4. O, J., et al., Thermodinamyc behavior of $\mathrm{Ca}_{3} \mathrm{Co}_{3.93+x} \mathrm{O}_{9+\delta}$ Ceramics. Ceramics-Silikáty, 2012. 56(2): p. 139-144.

5. Paul, B., J. Lu, and P. Eklund, Nanostructural Tailoring to Induce Flexibility in Thermoelectric $\mathrm{Ca}_{3} \mathrm{Co}_{4} \mathrm{O}_{9}$ Thin Films. ACS Applied Materials \& Interfaces, 2017. 9(30): p. 25308-25316.

6. Nagasawa, K., et al., $\mathrm{Ca}_{3} \mathrm{Co}_{4} \mathrm{O}_{9-\delta}:$ A Thermoelectric Material for SOFC Cathode. Chemistry of Materials, 2009. 21(19): p. 4738-4745.

7. Yang, G., Q. Ramasse, and R.F. Klie, Direct measurement of charge transfer in thermoelectric $\mathrm{Ca}_{3} \mathrm{Co}_{4} \mathrm{O}_{9}$. Physical Review B, 2008. 78(15): p. 153109.

8. Lin, Y.-H., et al., High-temperature electrical transport behaviors in textured $\mathrm{Ca}_{3} \mathrm{Co}_{4} \mathrm{O}_{9}$ based polycrystalline ceramics. Applied Physics Letters, 2009. 94(7): p. 072107.

9. Miyazaki, Y., et al., Modulated Structure of the Thermoelectric Compound $\left[\mathrm{Ca}_{2} \mathrm{CoO}_{3}\right]_{0.62} \mathrm{CoO}_{2}$. Journal of the Physical Society of Japan, 2002. 71(2): p. 491-497.

10. Delorme, F., et al., Synthesis of thermoelectric $\mathrm{Ca}_{3} \mathrm{Co}_{4} \mathrm{O}_{9}$ ceramics with high $\mathrm{ZT}$ values from a Co ${ }^{I I} \mathrm{Co}^{I I I}$-Layered Double Hydroxide precursor. Materials Research Bulletin, 2012. 47(11): p. 3287-3291.

11. Guilmeau, E., et al., Thermoelectric properties-texture relationship in highly oriented $\mathrm{Ca}_{3} \mathrm{Co}_{4} \mathrm{O}_{9}$ composites. Applied Physics Letters, 2004. 85(9): p. 1490-1492.

12. Fujii, S. and M. Yoshiya, Manipulating Thermal Conductivity by Interfacial Modification of Misfit-Layered Cobaltites $\mathrm{Ca}_{3} \mathrm{Co}_{4} \mathrm{O}_{9}$. Journal of Electronic Materials, 2016. 45(3): p. 1217-1226.

13. Butt, S., et al., Enhancement of thermoelectric performance in $\mathrm{Cd}$-doped $\mathrm{Ca}_{3} \mathrm{Co}_{4} \mathrm{O}_{9}$ via spin entropy, defect chemistry and phonon scattering. Journal of Materials Chemistry A, 2014. 2(45): p. 19479-19487.

14. Masset, A.C., et al., Misfit-layered cobaltite with an anisotropic giant magnetoresistance: $\mathrm{Ca}_{3} \mathrm{Co}_{4} \mathrm{O}_{9}$ Physical Review B, 2000. 62(1): p. 166-175.

15. Raveau, B. and M. Seikh, Cobalt oxides: from crystal chemistry to physics. 2012: John Wiley \& Sons.

16. Amin, B., U. Eckern, and U. Schwingenschlögl, Thermoelectric properties of the misfit cobaltate $\mathrm{Ca}_{3} \mathrm{Co}_{4} \mathrm{O}_{9}$. Applied Physics Letters, 2017. 110(23): p. 233505.

17. Tang, G., et al., Ultralow thermal conductivity and thermoelectric properties of carbon nanotubes doped $\mathrm{Ca}_{3} \mathrm{Co}_{4} \mathrm{O}_{9+\delta}$. Ceramics International, 2015. 41(1, Part B): p. 961-965.

18. Saini, S., et al., Terbium Ion Doping in $\mathrm{Ca}_{3} \mathrm{Co}_{4} \mathrm{O}_{9}$ : A Step towards High-Performance Thermoelectric Materials. Scientific Reports, 2017. 7: p. 44621.

19. Nong, N.V., C.-J. Liu, and M. Ohtaki, High-temperature thermoelectric properties of late rare earth-doped $\mathrm{Ca}_{3} \mathrm{Co}_{4} \mathrm{O}_{9+\delta}$. Journal of Alloys and Compounds, 2011. 509(3): p. 977981. 
20. Klyndyuk, A.I. and I.V. Matsukevich, Synthesis and properties of $\mathrm{Ca}_{2.8} \mathrm{Ln}_{0.2} \mathrm{Co}_{4} \mathrm{O}_{9+\delta}(\mathrm{Ln}$ $=\mathrm{La}, \mathrm{Nd}, \mathrm{Sm}, \mathrm{Tb}-\mathrm{Er}$ ) solid solutions. Inorganic Materials, 2012. 48(10): p. 1052-1057.

21. Pei, J., et al., High temperature transport and thermoelectric properties of $\mathrm{Ca}_{3-x} \mathrm{Er}_{x} \mathrm{Co}_{4} \mathrm{O}_{9+\delta .}$. Physica B: Condensed Matter, 2011. 406(3): p. 571-574.

22. Chen, J.L., et al., X-ray absorption spectroscopy studies of $\mathrm{Ca}_{2.9} \mathrm{Ln}_{0.1} \mathrm{Co}_{4} \mathrm{O}_{9+\delta}(\mathrm{Ln}=\mathrm{Ca}, \mathrm{Dy}$, $\mathrm{Ho}$, Er and Lu). Journal of Alloys and Compounds, 2012. 529: p. 8-11.

23. Van Nong, N., et al., Enhancement of the Thermoelectric Performance of p-Type Layered Oxide $\mathrm{Ca}_{3} \mathrm{Co}_{4} \mathrm{O}_{9+\delta}$ Through Heavy Doping and Metallic Nanoinclusions. Advanced Materials, 2011. 23(21): p. 2484-2490.

24. Koumoto, K., et al., Thermoelectric Ceramics for Energy Harvesting. Journal of the American Ceramic Society, 2013. 96(1): p. 1-23.

25. Lin, Y., et al., Preparation of $\mathrm{AgNPs} / \mathrm{Ca}_{3} \mathrm{Co}_{4} \mathrm{O}_{9}$ nanocomposites with enhanced thermoelectric performance. Materials Today Communications, 2016. 6: p. 44-49.

26. Park, K., D.A. Hakeem, and J.S. Cha, Synthesis and structural properties of thermoelectric $\mathrm{Ca}_{3-x} \mathrm{Ag}_{x} \mathrm{Co}_{4} \mathrm{O}_{9+\delta}$ powders. Dalton Transactions, 2016. 45(16): p. 6990-6997.

27. Zhang, F.P., et al., Texture and high temperature transport properties of $\mathrm{Ag}_{x} \mathrm{Ca}_{3-} \mathrm{Co}_{4} \mathrm{O}_{9}$ $(0 \leq x \leq 0.6)$ compounds. Journal of Alloys and Compounds, 2009. 477(1): p. 543-546.

28. Kahraman, F., et al., Enhancement of mechanical and thermoelectric properties of $\mathrm{Ca}_{3} \mathrm{Co}_{4} \mathrm{O}_{9}$ by $\mathrm{Ag}$ addition. Journal of the European Ceramic Society, 2015. 35(14): p. 38353841.

29. Wang, Y., et al., Comparison of the high temperature thermoelectric properties for Agdoped and $\mathrm{Ag}$-added $\mathrm{Ca}_{3} \mathrm{Co}_{4} \mathrm{O}_{9}$. Journal of Alloys and Compounds, 2009. 477(1): p. 817821.

30. Song, Y., et al., Synthesis and thermoelectric power factor of $\left(\mathrm{Ca}_{0.95} \mathrm{Bi}_{0.05}\right)_{3} \mathrm{Co}_{4} \mathrm{O}_{9} / \mathrm{Ag}$ composites. Materials Chemistry and Physics, 2009. 113(2): p. 645-649.

31. Song, Y. and C.-W. Nan, High temperature transport properties of Ag-added $\left(\mathrm{Ca}_{0.975} \mathrm{La}_{0.025}\right)_{3} \mathrm{Co}_{4} \mathrm{O}_{9}$ ceramics. Physica B: Condensed Matter, 2011. 406(14): p. 29192923.

32. Lu, Y., et al. Effects of Ag Addition on High Temperature Thermoelectric Properties of $\left(\mathrm{Ca}_{0 .}{ }_{9} \mathrm{Gd}_{0.1}\right)_{3} \mathrm{CO}_{4} \mathrm{O}_{9}$ Ceramics. Trans Tech Publ.

33. Zheng, Y., et al., High-temperature thermoelectric properties of $\mathrm{Ag}_{x} \mathrm{Y}_{y} \mathrm{Ca}_{2.8} \mathrm{Co}_{4} \mathrm{O}_{9+\delta}$ ceramics. Bulletin of Materials Science, 2014. 37(5): p. 963-967.

34. Zhang, F.P., Q.M. Lu, and J.X. Zhang, Modified texture and high temperature transport properties of doubly substituted $\mathrm{Ba}_{x} \mathrm{Ag}_{y} \mathrm{Ca}_{2.8} \mathrm{Co}_{4} \mathrm{O}_{9}$ thermoelectric oxide. Physica $\mathrm{B}$ : Condensed Matter, 2009. 404(16): p. 2142-2145.

35. Gupta, R.K., et al., The effect of $\mathrm{ZrO}_{2}$ dispersion on the thermoelectric power factor of $\mathrm{Ca}_{3} \mathrm{Co}_{4} \mathrm{O}_{9}$. Physica B: Condensed Matter, 2016. 483: p. 48-53.

36. Carvillo, P., et al., Thermoelectric Performance Enhancement of Calcium Cobaltite through Barium Grain Boundary Segregation. Inorganic Chemistry, 2015. 54(18): p. 90279032.

37. Boyle, C., et al., Grain boundary segregation and thermoelectric performance enhancement of bismuth doped calcium cobaltite. Journal of the European Ceramic Society, 2016. 36(3): p. 601-607.

38. Boyle, C., et al., Competing dopants grain boundary segregation and resultant seebeck coefficient and power factor enhancement of thermoelectric calcium cobaltite ceramics. Ceramics International, 2017. 43(14): p. 11523-11528. 
39. Tang, G.D., et al., Enhanced thermoelectric properties of $\mathrm{Ca}_{3} \mathrm{Co}_{4} \mathrm{O}_{9+\delta}$ by $\mathrm{Ni}$, Ce co-doping. Ceramics International, 2015. 41(5, Part B): p. 7115-7118.

40. Yang, W., et al., Effects of $\mathrm{Lu}$ and Ni Substitution on Thermoelectric Properties of $\mathrm{Ca}_{3} \mathrm{Co}_{4} \mathrm{O}_{9+\delta}$. Journal of Electronic Materials, 2016. 45(8): p. 4171-4176.

41. Park, K., et al., Thermoelectric transport properties of $\mathrm{Ca}_{3} \mathrm{Co}_{4-x} \mathrm{Ni}_{x} \mathrm{O}_{9+\delta}$ oxide materials. Journal of the Korean Physical Society, 2016. 68(1): p. 138-142.

42. $\mathrm{Wu}, \mathrm{N}$., et al., Effects of Yttrium and Iron co-doping on the high temperature thermoelectric properties of $\mathrm{Ca}_{3} \mathrm{Co}_{4} \mathrm{O}_{9+\delta}$. Journal of Alloys and Compounds, 2015. 638: p. 127-132.

43. Butt, S., et al., High-temperature thermoelectric properties of La and Fe co-doped CaCo-O misfit-layered cobaltites consolidated by spark plasma sintering. Journal of Alloys and Compounds, 2014. 588: p. 277-283.

44. Huang, C.S., et al., Enhanced thermoelectric figure of merit through electrical and thermal transport modulation by dual-doping and texture modulating for $\mathrm{Ca}_{3} \mathrm{Co}_{4} \mathrm{O}_{9+\delta}$ oxide materials. Journal of Alloys and Compounds, 2016. 687: p. 87-94.

45. Zhang, F.P., et al., Effects of Pr doping on thermoelectric transport properties of $\mathrm{Ca}_{3-x} \mathrm{Pr}_{x} \mathrm{Co}_{4} \mathrm{O}_{9}$. Solid State Sciences, 2011. 13(7): p. 1443-1447.

46. Liu, Z.Y., et al., Enhanced electrical transport by texture modulation and co-doping for $\mathrm{Ca}_{3} \mathrm{Co}_{4} \mathrm{O}_{9+\delta}$ materials. Results in Physics, 2016. 6: p. 203-208.

47. Butt, S., et al., Enhanced thermoelectric performance of heavy-metals $(\mathrm{M}: \mathrm{Ba}, \mathrm{Pb})$ doped misfit-layered ceramics: $\left(\mathrm{Ca}_{2}{ }_{-} \mathrm{M}_{x} \mathrm{CoO}_{3}\right)_{0.62}\left(\mathrm{CoO}_{2}\right)$. Energy Conversion and Management, 2014. 83: p. 35-41.

48. Butt, S., et al., Enhancement of Thermoelectric Performance in Hierarchical Mesoscopic Oxide Composites of $\mathrm{Ca}_{3} \mathrm{Co}_{4} \mathrm{O}_{9}$ and $\mathrm{La}_{0.8} \mathrm{Sr}_{0.2} \mathrm{CoO}_{3}$. Journal of the American Ceramic Society, 2015. 98(4): p. 1230-1235.

49. Liu, H.Q., et al., Effect of $\mathrm{Gd}$-doping on thermoelectric properties of $\mathrm{Ca}_{3} \mathrm{Co}_{4} \mathrm{O}_{9+\delta}$ ceramics. Journal of Materials Science, 2008. 43(21): p. 6933-6937.

50. Liu, H.Q., et al., Thermoelectric properties of $\mathrm{Ca}_{3-x} \mathrm{Y}_{x} \mathrm{Co}_{4} \mathrm{O}_{9+\delta}$ ceramics. Journal of Physics and Chemistry of Solids, 2009. 70(3): p. 600-603.

51. Liu, H.Q., et al., Thermoelectric properties of $\mathrm{Gd}, \mathrm{Y}$ co-doped $\mathrm{Ca}_{3} \mathrm{Co}_{4} \mathrm{O}_{9+\delta}$. Current Applied Physics, 2009. 9(2): p. 409-413.

52. $\mathrm{Xu}, \mathrm{L} ., \mathrm{F} . \mathrm{Li}$, and $\mathrm{Y}$. Wang, High-temperature transport and thermoelectric properties of $\mathrm{Ca}_{3} \mathrm{Co}_{4-x} \mathrm{Ti}_{x} \mathrm{O}_{9}$. Journal of Alloys and Compounds, 2010. 501(1): p. 115-119.

53. $\mathrm{Hu}, \mathrm{X}$., et al., Atomic and electronic structure of $\mathrm{Ti}$ substitution in $\mathrm{Ca}_{3} \mathrm{Co}_{4} \mathrm{O}_{9}$. Journal of Applied Physics, 2016. 120(20): p. 205105.

54. Torres, M.A., et al., Decrease of electrical resistivity in $\mathrm{Ca}_{3} \mathrm{CO}_{4} \mathrm{O}_{9}$ thermoelectric ceramics by Ti doping. Journal of Materials Science: Materials in Electronics, 2015. 26(2): p. 815820.

55. Morimura, T., et al., Microstructures and Thermoelectric Properties of Sintered $\mathrm{Ca}_{3} \mathrm{Co}_{4} \mathrm{O}_{9}$-Based Oxide. Journal of Electronic Materials, 2012. 41(6): p. 1217-1221.

56. Bhaskar, A., Y.C. Huang, and C.-J. Liu, Improvement on the low-temperature thermoelectric characteristics of $\mathrm{Ca}_{3-x} \mathrm{Yb}_{x} \mathrm{Co}_{4} \mathrm{O}_{9+\delta}(0 \leq x \leq 0.10)$. Ceramics International, 2014. 40(4): p. 5937-5943.

57. Kiirat, G., et al., Decrease of $\mathrm{Ca}_{3} \mathrm{Co}_{4} \mathrm{O}_{9+\delta}$ thermal conductivity by $\mathrm{Yb}$-doping. Ceramics International, 2015. 41(10, Part A): p. 12529-12534. 
58. Song, X., et al., Significant enhancement of electrical transport properties of thermoelectric $\mathrm{Ca}_{3} \mathrm{Co}_{4} \mathrm{O}_{9+\delta}$ through $\mathrm{Yb}$ doping. Solid State Communications, 2012. 152(16): p. 1509-1512.

59. Pinitsoontorn, S., et al., Thermoelectric properties of transition metals-doped $\mathrm{Ca}_{3} \mathrm{Co}_{3.8} \mathrm{M}_{0.2} \mathrm{O}_{9+\delta}(\mathrm{M}=\mathrm{Co}, \mathrm{Cr}, \mathrm{Fe}, \mathrm{Ni}, \mathrm{Cu}$ and $\mathrm{Zn})$. Journal of Materials Science: Materials in Electronics, 2012. 23(5): p. 1050-1056.

60. Presečnik, M. and S. Bernik, Microstructural and thermoelectric properties of $\mathrm{WO}_{3}$-doped $\mathrm{Ca}_{3} \mathrm{Co}_{4} \mathrm{O}_{9}$ ceramics. Ceramics International, 2016. 42(14): p. 16103-16108.

61. Shikano, M. and R. Funahashi, Electrical and thermal properties of single-crystalline $\left(\mathrm{Ca}_{2} \mathrm{CoO}_{3}\right)_{0.7} \mathrm{CoO}_{2}$ with a $\mathrm{Ca}_{3} \mathrm{Co}_{4} \mathrm{O}_{9}$ structure. Applied Physics Letters, 2003. 82(12): p. 1851-1853.

62. Obata, K., et al., Grain-Oriented $\mathrm{Ca}_{3} \mathrm{Co}_{4} \mathrm{O}_{9}$ Thermoelectric Oxide Ceramics Prepared by Solid-State Reaction. Journal of Electronic Materials, 2013. 42(7): p. 2221-2226.

63. Sotelo, A., et al., Improvement of thermoelectric properties of $\mathrm{Ca}_{3} \mathrm{Co}_{4} \mathrm{O}_{9}$ using soft chemistry synthetic methods. Journal of the European Ceramic Society, 2012. 32(10): p. 2415-2422.

64. Presečnik, M., J. de Boor, and S. Bernik, Synthesis of single-phase $\mathrm{Ca}_{3} \mathrm{Co}_{4} \mathrm{O}_{9}$ ceramics and their processing for a microstructure-enhanced thermoelectric performance. Ceramics International, 2016. 42(6): p. 7315-7327.

65. Delorme, F., et al., Thermoelectric properties of $\mathrm{Ca}_{3} \mathrm{Co}_{4} \mathrm{O}_{9}-\mathrm{Co}_{3} \mathrm{O}_{4}$ composites. Ceramics International, 2015. 41(8): p. 10038-10043.

66. $\mathrm{Wu}, \mathrm{N}$., et al., High temperature thermoelectric properties of $\mathrm{Ca}_{3} \mathrm{Co}_{4} \mathrm{O}_{9+\delta}$ by autocombustion synthesis and spark plasma sintering. Journal of the European Ceramic Society, 2014. 34(4): p. 925-931.

67. Soffientini, A., et al., Synthesis and Characterization of Bulk Nanostructured Thermoelectric $\mathrm{Ca}_{3} \mathrm{Co}_{4} \mathrm{O}_{9}$. Journal of Nanoscience and Nanotechnology, 2017. 17(3): p. 1674-1680.

68. Radhika, T., et al., Effect of Synthesis Conditions on Formation, Electrical Properties, and Seebeck Coefficient of p-Type $\mathrm{Ca}_{3} \mathrm{Co}_{4} \mathrm{O}_{9 \pm \delta}$ Thermoelectric Ceramics. Journal of Electronic Materials, 2017. 46(3): p. 1787-1793.

69. Chen, S., et al., Effect of precursor calcination temperature on the microstructure and thermoelectric properties of $\mathrm{Ca}_{3} \mathrm{Co}_{4} \mathrm{O}_{9}$ ceramics. Journal of Sol-Gel Science and Technology, 2012. 64(3): p. 627-636.

70. Karppinen, M., et al., Evidence for Oxygen Vacancies in Misfit-Layered Calcium Cobalt Oxide, $\left[\mathrm{CoCa}_{2} \mathrm{O}_{3}\right]_{q} \mathrm{CoO}_{2}$. Chemistry of Materials, 2004. 16(14): p. 2790-2793.

71. Noudem, J.G., et al., Thermoelectric $\mathrm{Ca}_{3} \mathrm{Co}_{4} \mathrm{O}_{9}$ ceramics consolidated by Spark Plasma sintering. Journal of Electroceramics, 2009. 22(1): p. 91-97.

72. Zhang, Y., J. Zhang, and Q. Lu, Synthesis of highly textured $\mathrm{Ca}_{3} \mathrm{Co}_{4} \mathrm{O}_{9}$ ceramics by spark plasma sintering. Ceramics International, 2007. 33(7): p. 1305-1308.

73. $\mathrm{Wu}, \mathrm{N}$., et al., Effects of Synthesis and Spark Plasma Sintering Conditions on the Thermoelectric Properties of $\mathrm{Ca}_{3} \mathrm{Co}_{4} \mathrm{O}_{9+\delta}$. Journal of Electronic Materials, 2013. 42(7): p. 2134-2142.

74. Liu, Y., et al., Preparation of $\mathrm{Ca}_{3} \mathrm{Co}_{4} \mathrm{O}_{9}$ and Improvement of its Thermoelectric Properties by Spark Plasma Sintering. Journal of the American Ceramic Society, 2005. 88(5): p. 13371340. 
75. Song, Y. and C.-W. Nan, Preparation of $\mathrm{Ca}_{3} \mathrm{Co}_{4} \mathrm{O}_{9}$ by polyacrylamide gel processing and its thermoelectric properties. Journal of Sol-Gel Science and Technology, 2007. 44(2): p. 139-144.

76. Kenfaui, D., et al., Texture, mechanical and thermoelectric properties of $\mathrm{Ca}_{3} \mathrm{Co}_{4} \mathrm{O}_{9}$ ceramics. Journal of Alloys and Compounds, 2010. 490(1): p. 472-479.

77. Bittner, M., et al., Porous $\mathrm{Ca}_{3} \mathrm{Co}_{4} \mathrm{O}_{9}$ with enhanced thermoelectric properties derived from Sol-Gel synthesis. Journal of the European Ceramic Society, 2017. 37(13): p. 3909-3915.

78. Gunes, M. and M. Ozenbas, Effect of grain size and porosity on phonon scattering enhancement of $\mathrm{Ca}_{3} \mathrm{Co}_{4} \mathrm{O}_{9}$. Journal of Alloys and Compounds, 2015. 626: p. 360-367.

79. Yin, Y., et al., Thermoelectric response of porous $\mathrm{Ca}_{3} \mathrm{Co}_{4} \mathrm{O}_{9}$ prepared by an eco-friendly technique. Ceramics International, 2017. 43(12): p. 9505-9511. 


\section{Chapter 3. Research Objectives}

The overall objective of the present work is the improvement of the ZT value and TE performance of polycrystalline $\mathrm{Ca}_{3} \mathrm{Co}_{4} \mathrm{O}_{9}$. Nonetheless, a key impediment for enhancing the TE performance is the interconnected material properties (Seebeck, electrical and thermal conductivity). In fact, a high Seebeck value typically leads to a high electrical resistivity, and the other way around. While a high electrical conductivity results in a high thermal conductivity, due to charge carriers carrying heat energy during the electrical conduction. In addition, the $\mathrm{Ca}_{3} \mathrm{Co}_{4} \mathrm{O}_{9}$ ceramics exhibit low electrical resistivity on the polycrystalline material due to random orientation of the crystals due to poor carrier mobility; a low Seebeck coefficient due to high carrier concentration inherent of oxide semiconductors; and a relatively high thermal conductivity due to light atomic mass elements and lack of phonon scattering events in the $\mathrm{Ca}_{3} \mathrm{Co}_{4} \mathrm{O}_{9}$.

Despite those limitations, it is of special importance the layered crystal structure of the bulk $\mathrm{Ca}_{3} \mathrm{Co}_{4} \mathrm{O}_{9}$ where each layer can play its own role in creating a specific purpose. Therefore, both the electron and phonon transport can be independently controlled to achieve a high TE performance of the ZT.

A lower resistivity could be achieved by improving the crystal texture (due to an increased carrier mobility) of polycrystalline $\mathrm{Ca}_{3} \mathrm{Co}_{4} \mathrm{O}_{9}$ with special processing (e.g. Spark plasma sintering and hot pressing) or by chemistry modification (e.g. cationic stoichiometric substitution and/or non-stoichiometric addition). Achieving a high TE performance without the use of special equipment or processes could reduce the material processing cost. Therefore, the chemistry modification to improve the electron transport properties is desirable in this assessment. 
Moreover, the Seebeck coefficient of the bulk ceramics could be improved (due to a reduced carrier concentration) with carrier filtering mechanisms by the segregation of the dopants at grain boundaries or with the increased grain boundary density. Both effects serve as filters of major number of low energy carriers which leads in having only high energy carrier that produce large Seebeck values. Additionally, it is possible to tune the Seebeck coefficient by substituting the $\mathrm{Ca}^{2+}$ in $\mathrm{Ca}_{3} \mathrm{Co}_{4} \mathrm{O}_{9}$ by superior valance state ions. With the chemistry modification the concentration of $\mathrm{Co}^{4+}$ in the $\mathrm{Ca}_{3} \mathrm{Co}_{4} \mathrm{O}_{9}$ could be reduced leading to a decrease in carrier concentration and an improved Seebeck value.

The thermal conductivity could be lowered by increasing the phonon scattering events in the polycrystalline $\mathrm{Ca}_{3} \mathrm{Co}_{4} \mathrm{O}_{9}$. This could be achieved with the inclusion of heavy elements, by increasing the grain boundary density or by replacing the host ions with elements with high atomic number element(Z).

Each chapter discusses the specific experimental procedure utilized and the material characterization. In addition, the details of the following chapters focus on the aforementioned strategies to enhance the thermoelectric properties by single and dual doping.

In chapter 5, Potassium is introduced to the polycrystalline $\mathrm{Ca}_{3} \mathrm{Co}_{4} \mathrm{O}_{9}$ by chemistry modification via non-stoichiometric addition. The motivation for this dopant is to get a lower resistivity by improving the crystal texture (and carrier mobility) with the chemistry modification. Additionally, since $\mathrm{Ba}^{2+}$ with similar size than $\mathrm{K}^{+}$has been reported to segregate at grain boundaries and improve the thermoelectric performance, thus, it is expected for Potassium to improve the Seebeck values(decreasing carrier concentration) due to the carrier filtering mechanisms at grain boundaries caused by segregation of the dopant. 
In chapter 6, detail description of Potassium and Bismuth doped $\mathrm{Ca}_{3} \mathrm{Co}_{4} \mathrm{O}_{9}$. The motivation of dual doping polycrystalline $\mathrm{Ca}_{3} \mathrm{Co}_{4} \mathrm{O}_{9} \mathrm{Bi}_{x} \mathrm{~K}_{\mathrm{y}}$ is to achieve a lower resistivity by improving the crystal texture (and carrier mobility) using dual non-stoichiometric addition. While the inclusion of $\mathrm{Bi}$ having a heavier atomic mass than the host ions in the polycrystalline $\mathrm{Ca}_{3} \mathrm{Co}_{4} \mathrm{O}_{9} \mathrm{Bi}_{\mathrm{x}} \mathrm{K}_{\mathrm{y}}$ is projected to decrease the thermal conductivity. Previous research has shown that the inclusion of Bi could improve the TE performance and develops crystal texture and grain growth in $\mathrm{Ca}_{3} \mathrm{Co}_{4} \mathrm{O}_{9}$. In addition, a secondary motivation would be to further research on the dual doping systems with different elements which have exhibited previously selective segregation and improvement of TE.

In chapter 7, the cationic substitution by heavy Terbium into $\mathrm{Ca}_{3-\mathrm{x}} \mathrm{Tb}_{\mathrm{x}} \mathrm{Co}_{4} \mathrm{O}_{9}$ is explored. The main motivation of selecting Terbium as a dopant is the capability to provide more phonon scattering events to lower the thermal conductivity. There is numerous research on rare earth elements as dopant to improve the TE performance of $\mathrm{Ca}_{3} \mathrm{Co}_{4} \mathrm{O}_{9}$. However, none of them have discovered the increasing grain boundary density with the reduced grain size. The increase of grain boundary density could simultaneously improve the Seebeck by filtering charge carriers and lower the thermal conductivity by scattering phonons.

In chapter $\mathbf{8}$, it is discussed the dual doping approach utilizing the cationic substitution by heavy Terbium with simultaneous non-stoichiometric addition of Potassium for different $\mathrm{Ca}_{3}$ ${ }_{x} \mathrm{~Tb}_{x} \mathrm{Co}_{4} \mathrm{O}_{9} \mathrm{~K}_{\mathrm{y}}$ concentrations. The motivation for selecting these dual doping elements consist in its specific characteristics as solo dopants. First, Terbium could provide a higher oxidation state $\mathrm{Tb}^{3+} / \mathrm{Tb}^{4+}$ element to replace the $\mathrm{Ca}^{2+}$ and in turn reduce the carrier concentration to enhance $S$. Moreover, the oversized Potassium atom could be segregated at the grain boundaries and further boost the $S$ of the bulk material. Second, adding Potassium into $\mathrm{Ca}_{3-\mathrm{x}} \mathrm{Tb}_{\mathrm{x}} \mathrm{Co}_{4} \mathrm{O}_{9}$ could improve the electrical transport performance. Additionally, the smaller ionic radii of Terbium in conjunction 
with the oversized ionic radii of Potassium could also play an important role in the microstructure of the bulk $\mathrm{Ca}_{3} \mathrm{Co}_{4} \mathrm{O}_{9}$ and could provide with more evidence of the grain boundary engineering.

In chapter 9, a comprehensive explanation on the $\mathrm{Ca}_{3-\mathrm{x}} \mathrm{Tb}_{\mathrm{x}} \mathrm{Co}_{4} \mathrm{O}_{9} \mathrm{Bi}_{\mathrm{y}}$ is given. $\mathrm{Bi}$ has shown to be a good dopant to improve the TE performance on bulk $\mathrm{Ca}_{3} \mathrm{Co}_{4} \mathrm{O}_{9}$. $\mathrm{Bi}$ alone as dopant $\mathrm{Bi}$ is segregated at the intergranular sites in $\mathrm{Ca}_{3} \mathrm{Co}_{4} \mathrm{O}_{9}$ this could potentially facilitate the development of crystal texture and grain growth due to faster diffusion of grain boundaries. Additionally, $\mathrm{Tb}$ with smaller ionic radii and $\mathrm{Bi}$ with larger ionic radii could facilitate the material engineering to control the intergranular and concurrent intragranular segregation and/or depletion at the grain boundaries. Further motivation in this approach is to further research on the dual doping systems and the microstructure evolution with different elements. 


\section{Chapter 4. General Experimental Procedure Utilized in Chapters 5 to 9}

\section{4. $1 \mathrm{Ca}_{3} \mathrm{Co}_{4} \mathrm{O}_{9}$ thermoelectric ceramic synthesis}

Polycrystalline ceramic samples were synthesized by the conventional chemical sol-gel route. The precursor powders were obtained mixing the chemical reagents according to the stoichiometric ratios. $\mathrm{Ca}\left(\mathrm{NO}_{3}\right)_{2} \cdot 4 \mathrm{H}_{2} \mathrm{O}\left(99 \%\right.$, Acros Organics), $\mathrm{Co}\left(\mathrm{NO}_{3}\right)_{2} \cdot 6 \mathrm{H}_{2} \mathrm{O}(99 \%$, Acros Organics), plus the specific doping element such as $\mathrm{Tb}\left(\mathrm{NO}_{3}\right)_{3} \cdot 6 \mathrm{H}_{2} \mathrm{O}(99.9 \%$, Strem Chemicals), $\mathrm{KNO}_{3}\left(99 \%\right.$, Alfa Aesar), and $\mathrm{Bi}\left(\mathrm{NO}_{3}\right)_{3} \cdot 5 \mathrm{H}_{2} \mathrm{O}$ (98\%, Acros Organics), were dissolved in deionized water. Citric acid (BDH Chemical), ethylene glycol, and polyethylene glycol were used to polymerize the mixture, while nitric acid was added to the solution to facilitate the sol-gel synthesis, nitrate salts decomposition, and the new compound formation. The mixture was mechanically stirred at $353 \mathrm{~K}$ for $3 \mathrm{~h}$. The obtained sol-gel solution was ashed at $773 \mathrm{~K}$ for $2 \mathrm{hr}$ in air inside a box furnace. The resulting ashes were ball-milled in ethyl alcohol in a stage with zirconia balls and then dried at room temperature with a final manually ground stage after that. The fine ashes were calcined at $973 \mathrm{~K}$ for $4 \mathrm{~h}$ in a tube furnace with a constant oxygen flow. The final precursor powders were uniaxially pressed into round pellets under a $1 \mathrm{Gpa}$ pressure at room temperature. The bulk samples were sintered at temperature $1233 \mathrm{~K}$ in a tube furnace with a constant oxygen flow and then cut into rectangular pellets to obtain the final desired sample for either the electrical or thermal measurements. Figure 4-1 shows the procedure of fabrication polycrystalline ceramics pellets with the precursor powders made from the conventional chemical sol-gel route. 


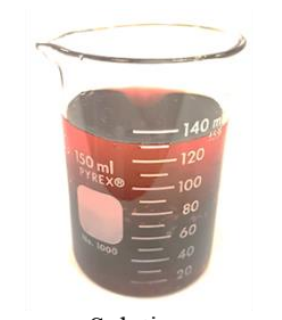

Solution

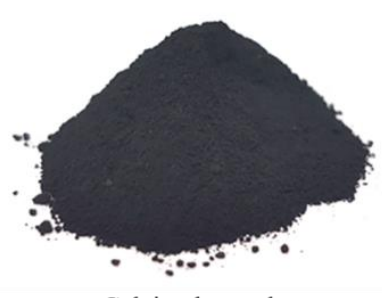

Calcined powder

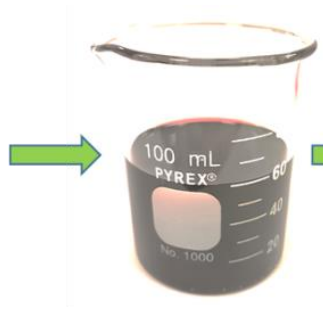

Sol-gel

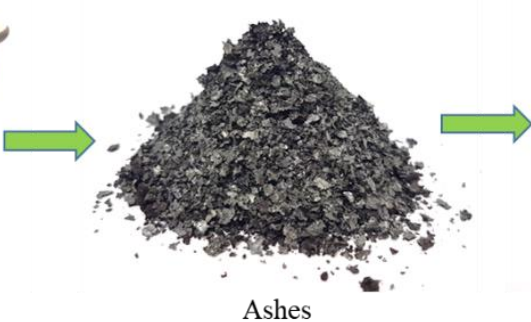

Ashes

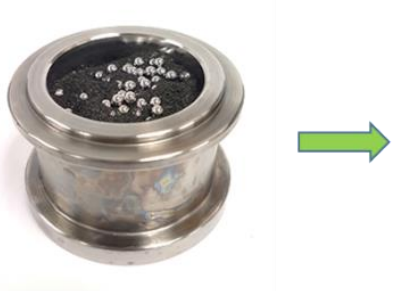

Ball-mill

Figure 4-1: procedure of fabrication polycrystalline ceramics pellets with the precursor powders made from the conventional chemical sol-gel route.

\section{2 Electrical and thermal properties measurement}

The electrical resistivity $(\rho)$ and absolute Seebeck coefficient $(S)$ were measured by the fourterminal method using a Linseis LSR-1100 unit. The measurements were performed direction to the pressed direction from $323 \mathrm{~K}$ to $1073 \mathrm{~K}$ under a low pressure He environment. Figure 4-2 shows the working principle for the Seebeck and resistivity measurements of the LSR unit.
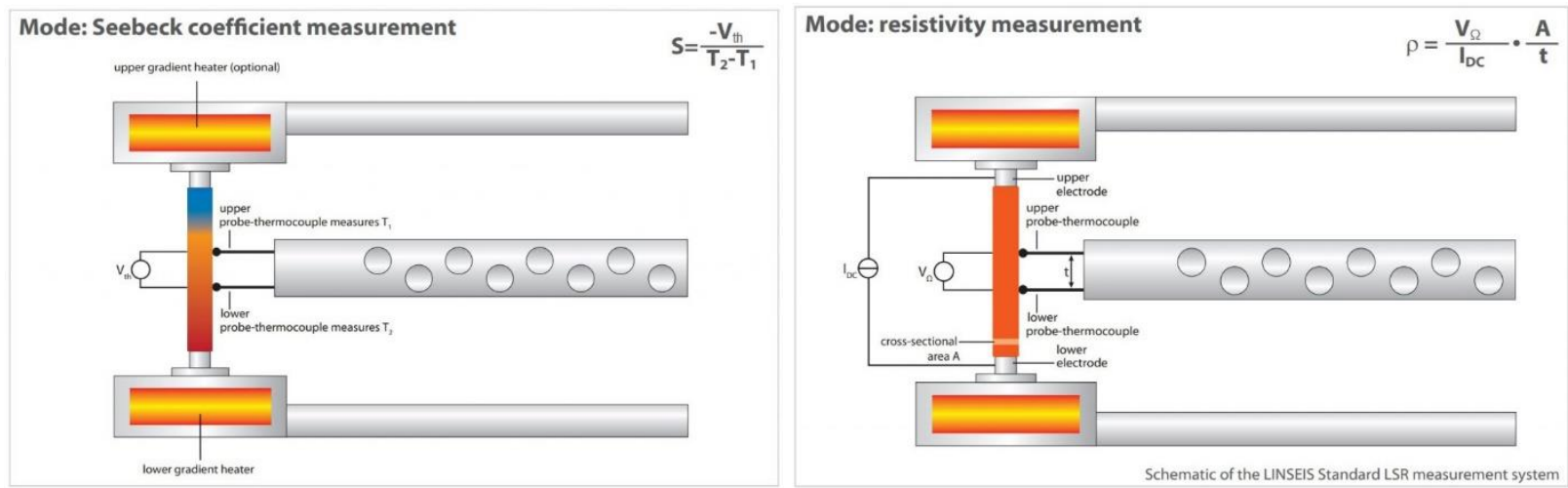

Figure 4-2: Principle of the Seebeck and resistivity measurement in the LSR unit. Retrieved from: linseis.com/en/products/thermoelectrics/lsr-3/

The thermal conductivity was calculated using the equation $\kappa=\lambda C_{p} \rho m$, where $C_{p}, \lambda$, and $m$ are the specific heat, thermal diffusivity, and mass density, respectively. The $C p$ and $\lambda$ values were 
obtained from $323 \mathrm{~K}$ to $1073 \mathrm{~K}$ using a Linseis Laser Flash Analyzer 1200. The measurements were performed perpendicular to the pressed direction under a low-pressure air environment.

\section{3 Microstructure and nanostructure imaging with TEM and SEM}

The cross-section morphology of the sintered pellets was observed using a Hitachi-S4700F Scanning Electron Microscope (SEM) unit. Sintered samples were mounted to a SEM sample holder using silver paste with no other further processing or coating utilized. The samples for transmission electron microscopy (TEM) were prepared by mechanically polishing and ion milling in a liquid-nitrogen-cooled holder. Electron diffraction, diffraction contrast, and high-resolution TEM imaging were performed in a JEM-2100 operated at $200 \mathrm{kV}$. The chemical analysis was carried out using energy dispersive spectroscopy (EDS) under TEM. High-angle annular dark-field (HAADF) Z-contrast imaging and nanoscale chemistry analysis were performed using an Aberration-Corrected $200 \mathrm{kV}(\mathrm{JEOL}$ 2100F) STEM with the inner cut-off angle of the HAADF detector set at $>52 \mathrm{mrad}$.

\section{4 XRD crystal structure analysis}

The $\mathrm{Ca}_{3} \mathrm{Co}_{4} \mathrm{O}_{9}$ polycrystalline bulk sample of the present study possess a preferred orientation of crystal grains. Therefore, sintered pellets are grounded to small particles which lead crystallites to adopt all possible orientations randomly and, thus, be able to obtain diffraction peaks of all $h k l$ planes. X-Ray powder Diffraction (XRD) analysis is performed using a PANatycal X'Pert Pro with a step size of 0.017 for the $2 \theta$ angle. Powders from the sintered pellets are placed in the center of the stages with a similar amount of material. A smooth thin flat surface is created by pressing down with a glass slide. Stage spinning while performing measurements is activated to get signal of more particles. Specimen preparation in this way reduces the error associated with sample displacement, sample transparency, preferred orientation, and other sources of error. Furthermore, 
all specimens are characterized with the same parameters and at the same time, meaning that all 5 different concentrations are scanned in the same session. The above XRD sample preparation and experimental set up were aimed to minimize the error of the individual measurements.

The calculation of lattice parameters is done with results acquired from the analysis performed by the PANatycal X'Pert Pro. The values of lattice parameters are obtained by solving the equation of interplanar spacing. For a monoclinic crystal system, the d spacing between adjacent $h k l$ planes is given by:

$$
\frac{1}{d^{2}}=\frac{1}{\sin ^{2} \beta}\left(\frac{h^{2}}{a^{2}}+\frac{k^{2} \sin ^{2} \beta}{b^{2}}+\frac{l^{2}}{c^{2}}-\frac{2 h l \cos \beta}{a c}\right)
$$

where

$$
d_{h k l}=\frac{\lambda}{2 \sin \theta_{h k l}}
$$

Thus, to study the effect of lattice parameter changes introduced by doping through the above mentioned XRD procedure, the only error associated with the calculations is the selection of the position of the Bragg angle at the corresponding $h k l$ peak. Five different peaks are needed in order to obtain the five lattice parameters. Furthermore, some peaks will give redundant information, therefore, the use of the peaks related to the planes $001,020,220,202$, and 110 are necessary to find all values. To do so, the $2 \theta_{h k l}$ angle value of the matching $h k l$ peak is selected manually by choosing the corresponding highest peak intensity. In addition, the $2 \theta_{h k l}$ angle value has also been selected by means of the Fullprof software, such software uses the FWHM to calculate the position of the Bragg angle matching the $h k l$ peak. Subsequently, the $2 \theta_{h k l}$ angle values obtained by the software are employed to solve the equation of interplanar spacing in order to obtain the lattice parameters. 
To better quantify the crystallographic texture orientation degree of samples, the texture fraction (Lotgering factor) of the $\{001\}$ planes was calculated utilizing the intensities of the XRD peaks obtained at room temperature. The Lotgering factor $(L F)$ is defined as $L F=\left(P_{l}-P_{0}\right) /\left(1-P_{0}\right)$, where $P_{l}=\sum I_{0 o l} \sum I_{h k l}$ and $P_{0}=\sum I_{0 o l}^{\prime} \sum I_{h k l}^{\prime}$ denotes the fraction of the summation of the peak intensities for the as sintered pellet sample(textured oriented) and the after-sintering ground sample (randomly oriented), respectively. A fraction close to the unity correspond to the best possible orientation. The integrated densities of the XRD peaks were obtained with Fullprof software. 


\section{Chapter 5. Impact of Potassium Addition on the Thermoelectric Performance of $\mathrm{Ca}_{3} \mathrm{Co}_{4} \mathrm{O}_{9}$ Ceramics}

\section{1 Improving electrical transport properties with oversize $\mathbf{K}^{+}$}

The effect of non-stoichiometric addition of potassium $\mathrm{K}^{+}$on the microstructure and thermoelectric performance of misfit layered calcium cobaltite $\mathrm{Ca}_{3} \mathrm{Co}_{4} \mathrm{O}_{9}$ ceramics is reported. The samples of present study are with designed nominal composition of $\mathrm{Ca}_{3} \mathrm{Co}_{4} \mathrm{O}_{9} \mathrm{~K}_{\mathrm{x}}(\mathrm{x}=0,0.05$, $0.1,0.15$, and 0.2$). \mathrm{K}^{+}$non-stoichiometric addition promotes the crystal growth and improve the grain alignment and crystal texture of micron sized grains. Nanostructure and chemistry analysis under TEM also reveal the segregation of the $\mathrm{K}^{+}$at the grain boundaries of $\mathrm{Ca}_{3} \mathrm{Co}_{4} \mathrm{O}_{9}$ grains with different c-axis, whereas the interior of the $\mathrm{Ca}_{3} \mathrm{Co}_{4} \mathrm{O}_{9}$ grain is free of the dopant $\mathrm{K}^{+}$. The electrical resistivity and the Seebeck Coefficient of $\mathrm{Ca}_{3} \mathrm{Co}_{4} \mathrm{O}_{9}$ ceramics were both affected by $\mathrm{K}^{+}$addition. Seebeck Coefficient increases with the increasing doping level of $\mathrm{K}^{+}$. For the polycrystalline sample with optimal doping chemistry of $\mathrm{Ca}_{3} \mathrm{Co}_{4} \mathrm{O}_{9} \mathrm{~K}_{0.1}$, at $320 \mathrm{~K}$, the sample reached the highest Thermoelectric power factor $930 \mu \mathrm{W} \mathrm{K} \mathrm{K}^{-2}$, which is 2.25 times higher than that of an already good performance $\left(412 \mu \mathrm{W} \mathrm{K}^{-2} \mathrm{~m}^{-1}\right)$ of that from baseline $\mathrm{Ca}_{3} \mathrm{Co}_{4} \mathrm{O}_{9}$. The $\mathrm{ZT}$ of the material is enhanced to a value of 0.42 for the optimal concentration. The present work demonstrates the $\mathrm{K}^{+}$ segregation at the grain boundary impact both the carrier concentration and carrier mobility for the polycrystalline $\mathrm{Ca}_{3} \mathrm{Co}_{4} \mathrm{O}_{9}$ ceramics. At optimal doping level, $\mathrm{K}^{+}$grain boundary segregation reduced the electrical resistivity and increase Seebeck coefficient simultaneously, resulting in large increase in the electrical power factor.

\section{2 Background \& motivation for $\mathrm{Ca}_{3} \mathrm{C}_{4} \mathrm{O}_{9} \mathrm{~K}_{x}$ material}

Thermoelectric (TE) power generation is one of the most prominent energy production for harnessing the waste heat, otherwise not be recovered, produced by diverse sectors, such as 
transportation $^{[1,2]}$, industrial ${ }^{[3,4]}$, and nuclear ${ }^{[5]}$. In comparison with other electricity power generation technology, TE device features no moving parts, maintenance free operation, an extended longevity. In addition, converting heat into electricity through the Seebeck effect ${ }^{[6-8]}$ is environmentally friendly with zero emission, thus overcoming the necessity of fossil fuels, and thereby decreasing greenhouse gases ${ }^{[2]}$.

There is a waste heat harnessing potential at high temperature ${ }^{[9]}$, notwithstanding to be capable to recover the high-temperature heat, TE materials must be thermostable and have high performance at elevated temperatures, preferably, materials must retain the TE properties at high temperatures in air. Several materials have been investigated as TE materials over the last years, whereas some of them possess rare and toxic materials that oxidize at high temperatures ${ }^{[10]}$. For instance, commercial TE materials such as BiSbTe already achieves peak figure of merit ZT values of $1.4^{[11]}$, nonetheless, the best performance sits at low temperature $(373 \mathrm{~K})$ making it not suitable for elevated temperatures application. Additionally, without the use of a protection coating the stability of conventional semiconductor TE materials is compromised at high temperatures leading to degradation of the essential TE properties due to changes in stoichiometry by sublimation, and material oxidation $^{[12]}$.

In contrast, metal oxides provide a big advantage for its low cost and great alternative to overcome thermostability at high temperatures ${ }^{[7]} \cdot \mathrm{Ca}_{3} \mathrm{Co}_{4} \mathrm{O}_{9}$ misfit layered calcium cobaltite has been one of the most promising p-type oxide materials for thermoelectric power generation ${ }^{[7,8,10}$, ${ }^{13-20]}$, and it possess good thermostability in air up to $1199 \mathrm{~K}^{[21]}$. Nevertheless, the large scale development of the $\mathrm{Ca}_{3} \mathrm{Co}_{4} \mathrm{O}_{9}$ has been largely neglected during the past decade because the currently low energy conversion efficiency of the polycrystalline material due to its low electrical power factor ${ }^{[7]}$. 
Synthesis process of calcium cobaltite is one important aspect to tune in order to obtain desirable TE properties of the material. Sol-gel chemical route is reported to significantly reduce the secondary phase content in $\mathrm{Ca}_{3} \mathrm{Co}_{4} \mathrm{O}_{9}$ compared to conventional solid state reaction ${ }^{[22]}$. Reduction of secondary phases lead to an increase in electrical conductivity and a corresponding increase of the electrical power factor. Furthermore, sol-gel route offers a homogenous composition of the sample and accurate control of the designed chemistry by either stoichiometric substitution or non-stoichiometric addition. Additionally, optimal temperatures for calcination ${ }^{[14]}$ and sintering ${ }^{[18]}$ stages have been previously reported in order to obtain the best performance of the bulk $\mathrm{Ca}_{3} \mathrm{Co}_{4} \mathrm{O}_{9}$.

Similarly, different fabrication methods have been investigated to positively influence the TE properties of the material. It has been reported that innovative techniques such as magnetic fields during the pressing stage of the material ${ }^{[20]}$ could positively influence the grain alignment and as consequence improve the electrical conductivity. Moreover, spark plasma sintering ${ }^{[19]}$ offers another alternative to enhance the material properties and reducing the sintering times and crystal alignment of the material. These techniques offer an interesting approach to enhance the electrical resistivity, nevertheless, they add complexity to the processing.

Ion doping via lattice substitution remains one of the most promising approaches to enhance the TE capabilities in polycrystalline $\mathrm{Ca}_{3} \mathrm{Co}_{4} \mathrm{O}_{9}$, particularly, partial substitution of the $\mathrm{Ca}^{2+}$. Doping $\mathrm{Yb}^{3+}{ }^{[13]}$ in the $\mathrm{Ca}^{2+}$ site is reported to enhance the electrical transport properties and Seebeck coefficient attaining an electrical power factor of $553 \mu \mathrm{W} \mathrm{K}^{-2} \mathrm{~m}^{-1}$. Additionally, Ag-rich regions and nanoinclusions enhance electrical conductivity and $\mathrm{S}$ simultaneously ${ }^{[16]} \cdot \mathrm{K}^{+}$ substitution in $\mathrm{Ca}^{2+}$ lattice site ${ }^{[17]}$ is reported to work as a phonon scattering to reduce thermal conductivity. Similarly, $\mathrm{La}^{3+}$ and $\mathrm{K}^{+}$substitution in $\mathrm{Ca}_{3} \mathrm{Co}_{4} \mathrm{O}_{9}$ ceramics are reported to increase 
both carrier concentration and carrier mobility ${ }^{[23]}$, improving the Seebeck coefficient and electrical conductivity, respectively. Nonetheless, there is insufficient detail in the effects on nonstoichiometric $\mathrm{K}^{+}$addition in bulk $\mathrm{Ca}_{3} \mathrm{Co}_{4} \mathrm{O}_{9}$.

More importantly, different doping elements segregated to the grain boundary has been reported in TE materials to significantly increase the electrical power factor ${ }^{[24-26]}$. In previous work has been demonstrated that $\mathrm{Bi}^{[15]}$ and $\mathrm{Ba}^{[27]}$ could be introduced into $\mathrm{Ca}_{3} \mathrm{Co}_{4} \mathrm{O}_{9}$ ceramics using either substitution or non-stoichiometric addition, respectively, to improve the texture of the crystal, and enhance the Seebeck coefficient and electrical conductivity. Substitution of $\mathrm{Bi}^{3+}$ is reported to be incorporated into the lattice and grain boundary. Moreover, non-stoichiometric addition of $\mathrm{Ba}^{2+}$ segregates only to the grain boundary, thus, resulting beneficial the nonstoichiometric approach rather than substitution. Furthermore, oxidation states are different for $\mathrm{Bi}^{3+}$ and $\mathrm{Ba}^{2+}$, still both elements segregates at the grain boundary when introduced into the $\mathrm{Ca}_{3} \mathrm{Co}_{4} \mathrm{O}_{9}$, suggesting that segregation is not valence state controlled. Based on those results, this paper proposes that grain boundary effect is mostly controlled by ionic size difference between the dopant and the $\mathrm{Ca}_{3} \mathrm{Co}_{4} \mathrm{O}_{9}$ lattice sites. A different oxidation state element $\left(\mathrm{K}^{+}\right)$with a large difference in ionic size in comparison with the $\mathrm{Ca}_{3} \mathrm{Co}_{4} \mathrm{O}_{9}$ lattice sites was selected as a dopant to analyze the grain boundary segregation effect in the bulk polycrystalline material. Specifically, emphasizing on the substantial increase in the Seebeck coefficient and the improvement in the crystal texture associated with the $\mathrm{K}^{+}$segregation at the $\mathrm{Ca}_{3} \mathrm{Co}_{4} \mathrm{O}_{9}$ grain boundary is discussed.

\section{3 Experimental procedure}

$\mathrm{Ca}_{3} \mathrm{Co}_{4} \mathrm{O}_{9} \mathrm{~K}_{x}(x=0,0.05,0.1,0.15$, and 0.2$)$ powders were prepared by the conventional chemical sol-gel route mixing the chemical reagents according to the stoichiometric ratios. $\mathrm{Ca}\left(\mathrm{NO}_{3}\right)_{2} \cdot 4 \mathrm{H}_{2} \mathrm{O}\left(99 \%\right.$, Acros Organics), $\mathrm{Co}\left(\mathrm{NO}_{3}\right)_{2} \cdot 6 \mathrm{H}_{2} \mathrm{O}$ (99\%, Acros Organics), and $\mathrm{KNO}_{3}$ 
(99\%, Alfa Aesar) were dissolved in deionized water. Citric acid (BDH Chemical), ethylene glycol (BDH Chemical), poly (ethylene) glycol (Acros Organics), and nitric acid (BDH Chemical) were added to the solution to facilitate the sol-gel synthesis and the new compound formation. Then the mixture was mechanically stirred for $3 \mathrm{~h}$ at $353 \mathrm{~K}$. The obtained sol-gel solution was ashed at 773 $\mathrm{K}$ for $2 \mathrm{hr}$ in air inside a box furnace. The resulting ashes were ball-milled in ethyl alcohol in a stage with zirconia balls and then dried at room temperature with a final manually ground stage after that. The fine ashes were calcined at $973 \mathrm{~K}$ for $4 \mathrm{~h}$ in a tube furnace with a constant oxygen flow. The final precursor powders were uniaxially cold pressed into round pellet shape under a 1 Gpa pressure at room temperature. The bulk samples were sintered at $1233 \mathrm{~K}$ in a tube furnace with a constant oxygen flow and then cut into rectangular pellets to obtain the final desired dimensions. The apparent densities for all bulk samples are listed in Table 5-1.

Table 5-1: Apparent density of the $\mathrm{Ca}_{3} \mathrm{Co}_{4} \mathrm{O}_{9} \mathrm{~K}_{x}$ samples.

\begin{tabular}{ll}
\hline $\mathrm{Ca}_{3} \mathrm{Co}_{4} \mathrm{O}_{9} \mathrm{~K}_{\mathrm{x}}$ & Average Apparent density $\left(\mathrm{g} / \mathrm{cm}^{3}\right)$ \\
\hline $\mathrm{x}=0$ & 3.883 \\
$\mathrm{x}=0.05$ & 4.119 \\
$\mathrm{x}=0.10$ & 4.424 \\
$\mathrm{x}=0.15$ & 4.339 \\
$\mathrm{x}=0.20$ & 4.265 \\
\hline
\end{tabular}

The electrical resistivity $(\rho)$ and absolute Seebeck (S) coefficient were measured by the fourterminal method using a Linseis LSR-1100 unit. Measurements were performed in the parallel direction to the pressed plane from $323 \mathrm{k}$ to $1073 \mathrm{~K}$ under a low pressure He environment. The characterization provides both $\mathrm{S}$ and $\rho$ of the material, as well as the determination of the electrical power factor. Thermal properties of the materials were characterized in vacuum using a Laser Flash Analyzer Linseis-1200. Measurements provided the thermal diffusivity and the specific heat in the parallel direction to the pressed plane within a temperature range of $298 \mathrm{~K}$ to $1073 \mathrm{~K}$. X- 
Ray powder Diffraction (XRD) analysis was performed using a PANatycal X'Pert Pro XRD unit for phase and lattice parameter identification purposes. Powders from the sintered samples were used in the XRD analysis using $\mathrm{Cu} \mathrm{K}$-alpha radiation, $45 \mathrm{kV}$ tension and $40 \mathrm{~mA}$ current at room temperature. Surface and cross-section morphology was inspected using Hitachi-S4700F Scanning Electron Microscope (SEM) unit. JEOL JEM-2100 Transmission Electron Microscope (TEM) equipped with Energy Dispersive X-Ray Spectroscopy (EDS) was employed to characterize the nanostructure and chemistry of samples inside the grain and in the grain boundary at atomic scale.

\section{4 Results and discussion}

The XRD patterns of $\mathrm{Ca}_{3} \mathrm{Co}_{4} \mathrm{O}_{9} \mathrm{~K}_{x}$ samples are shown in Figure 5-1. The data has been plotted with a slight offset for visualization. All XRD characteristic peaks can be indexed as those from the $\mathrm{Ca}_{3} \mathrm{Co}_{4} \mathrm{O}_{9}$ phase (PDF: \#23-110) with monoclinic symmetry. The patterns do not show any shift in the Bragg angle of the peaks for the different concentrations which indicates that the $\mathrm{K}^{+}$ does not modify the lattice parameters of $\mathrm{Ca}_{3} \mathrm{Co}_{4} \mathrm{O}_{9}$. Moreover, the representative diffraction peaks of secondary phase were not found in any of the doping concentrations.

Table 5-2: Lattice parameters $a, b_{1}, b_{2}, c$, and $\beta$ for $\mathrm{Ca}_{3} \mathrm{Co}_{4} \mathrm{O}_{9} K_{x}(x=0,0.05,0.1,0.15$, and 0.2) samples. $b_{1}$ and $b_{2}$ refer to b-axis lattice parameters in the rock-salt layer $\mathrm{Ca}_{2} \mathrm{CoO}_{3}$ and the

$\mathrm{CoO}_{2}$ layer, respectively.

\begin{tabular}{lccccc}
\hline $\mathrm{Ca}_{3} \mathrm{Co}_{4} \mathrm{O}_{9} \mathrm{~K}_{x}$ & $a(\AA)$ & $b_{1}(\AA)$ & $b_{2}(\AA)$ & $c(\AA)$ & $\beta$ \\
\hline$x=0$ & 4.909 & 4.525 & 2.753 & 10.516 & 99.540 \\
$x=0.05$ & 4.905 & 4.521 & 2.756 & 10.557 & 99.569 \\
$x=0.10$ & 4.904 & 4.523 & 2.756 & 10.575 & 99.471 \\
$x=0.15$ & 4.903 & 4.523 & 2.762 & 10.572 & 99.344 \\
$x=0.20$ & 4.902 & 4.521 & 2.763 & 10.550 & 99.317 \\
\hline
\end{tabular}




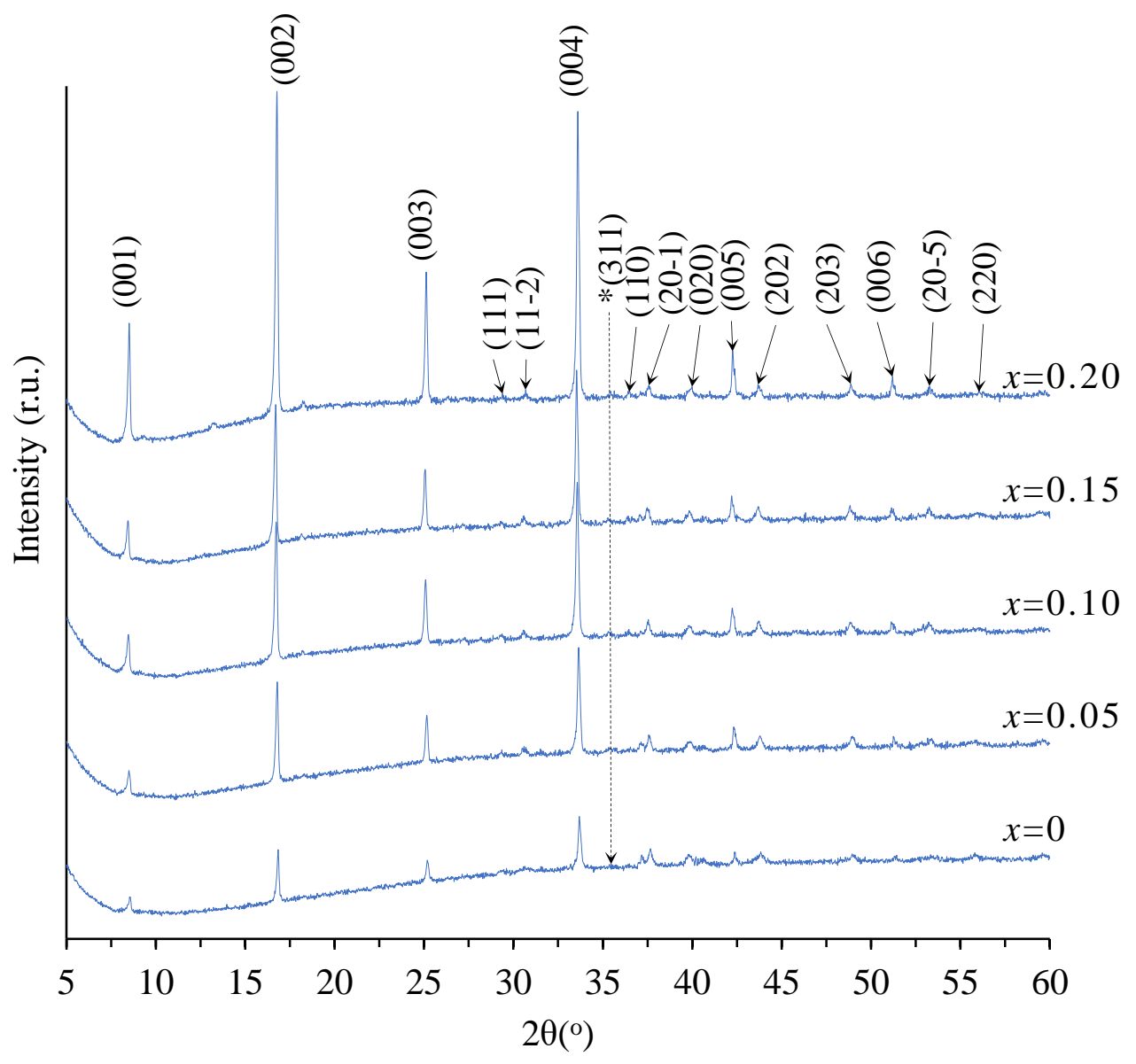

Figure 5-1: $\mathrm{XRD}$ powder diffraction patterns for the undoped $\mathrm{Ca}_{3} \mathrm{Co}_{4} \mathrm{O}_{9}$ and the doped $\mathrm{Ca}_{3} \mathrm{Co}_{4} \mathrm{O}_{9} \mathrm{~K}_{\mathrm{x}}(\mathrm{x}=0.05,0.1,0.15$, and 0.2$)$ samples.

The lattice parameter values for each sample are listed in Table 5-2, which confirms that the lattice remains unchanged. The small variations of the values listed in Table 5-2 is attributed to the inherent error associated with the experimental data.

Cross-sectional SEM images taken from the fractured surface of the $\mathrm{Ca}_{3} \mathrm{Co}_{4} \mathrm{O}_{9} \mathrm{~K}_{x}$ pellets are shown in Figure 5-2. With the increase in the potassium doping level, the size of the crystals gradually increase. Furthermore, it can also be observed the preference in the orientation for the optimum $\mathrm{Ca}_{3} \mathrm{Co}_{4} \mathrm{O}_{9} \mathrm{~K}_{0.1}$ concentration, the majority of the crystals have the c-axis of the monoclinic structure perpendicular to the pressed direction. The better orientation of the lamellas 
and the enhancement of the c-axis crystal texture make the TE properties better than the other concentrations since the grain alignment significantly improves the electrical properties of the material. Additionally, for higher doping levels the pellets display a more disoriented grains and the crystal texture fade.
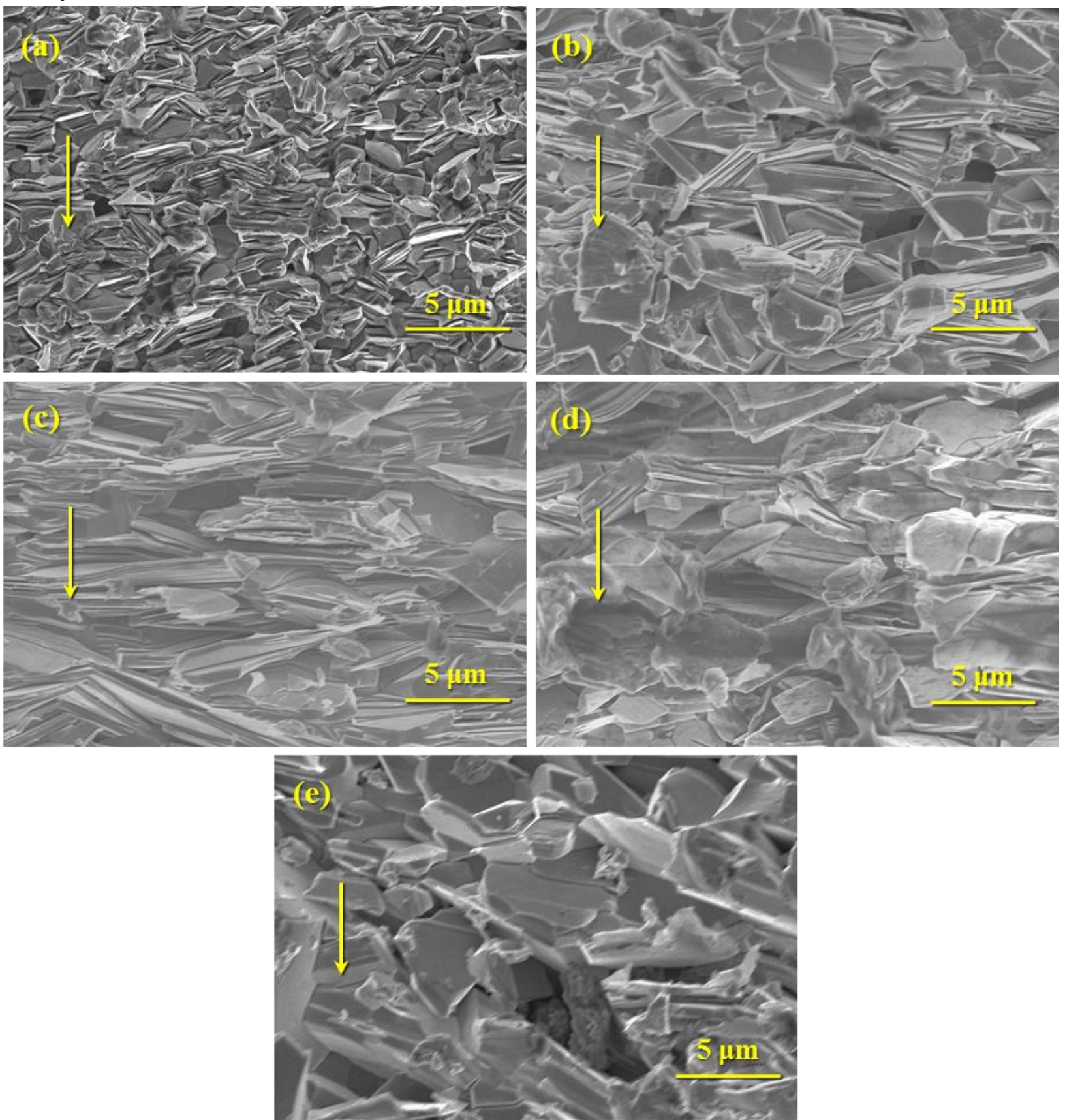

Figure 5-2:Cross-sectional SEM images from the fractured surface of $\mathrm{Ca}_{3} \mathrm{Co}_{4} \mathrm{O}_{9} \mathrm{~K}_{x}(x=0,0.05$, 0.1, 0.15, and 0.2) samples after sintering stage. (a) $\mathrm{Ca}_{3} \mathrm{Co}_{4} \mathrm{O}_{9},(b) \mathrm{Ca}_{3} \mathrm{Co}_{4} \mathrm{O}_{9} \mathrm{~K}_{0.05}$, (c) $\mathrm{Ca}_{3} \mathrm{Co}_{4} \mathrm{O}_{9} \mathrm{~K}_{0.10}, \quad(d) \mathrm{Ca}_{3} \mathrm{Co}_{4} \mathrm{O}_{9} \mathrm{~K}_{0.15},(e) \mathrm{Ca}_{3} \mathrm{Co}_{4} \mathrm{O}_{9} \mathrm{~K}_{0.20}$. 


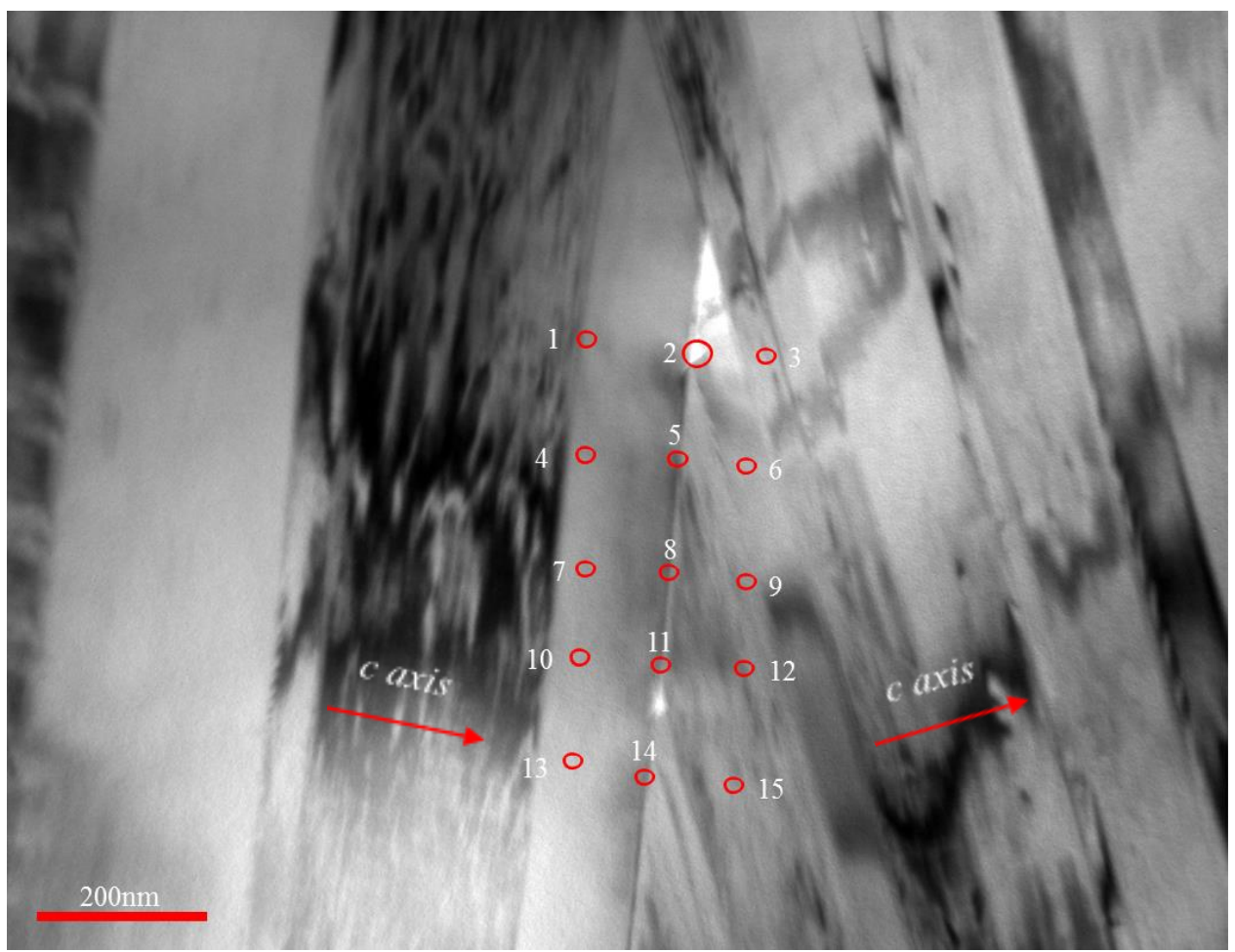

Figure 5-3: Low magnification TEM diffraction contrast image showing the EDS spot examinations performed at different location between two adjacent grains and the grain boundary for the nanostructure analysis of $\mathrm{Ca}_{3} \mathrm{Co}_{4} \mathrm{O}_{9} \mathrm{~K}_{0.1}$. Results are given in Table 5-3.

To further explore the effect of $\mathrm{K}^{+}$doping in the grain boundaries, TEM examination was performed on the sample $\mathrm{Ca}_{3} \mathrm{Co}_{4} \mathrm{O}_{9} \mathrm{~K}_{0.1}$ with the best TE properties. TEM image of Figure 5-3 illustrates the nanolamella structure of two adjacent grains for the $\mathrm{Ca}_{3} \mathrm{Co}_{4} \mathrm{O}_{9} \mathrm{~K}_{0.1}$ sample. The circled numbers represent the EDS spot subjected to examination, these spots correspond both to the interior of the grain and to the grain boundary between the adjacent grains. The atomic percent results for each EDS spot is summarized in Table 5-3. Further evidence supporting the absence of $\mathrm{K}^{+}$inside the lattice crystal is confirmed. Hence, EDS results are consistent with the information obtained by XRD analysis, the $\mathrm{Ca}_{3} \mathrm{Co}_{4} \mathrm{O}_{9}$ lattice is free of any $\mathrm{K}^{+}$, whereas at the grain boundaries a large amount of potassium prevails which indicates the boundary segregation of the doping element, supporting the fact that lattice parameters remained unchanged. 
Table 5-3: EDS spot examination for $\mathrm{Ca}_{3} \mathrm{Co}_{4} \mathrm{O}_{9} \mathrm{~K}_{0.1}$ sample showing the chemistry of the interior of the grain (spots 1, 3, 4, 6, 7, 9, 10, 12, 13, and 15) and between grain boundary areas (spots $2,5,8,11,14)$, respectively. The symbol (-) indicates the absence of the element in such spot.

\begin{tabular}{lccccccccccccccc}
\hline At $\%$ & 1 & 2 & 3 & 4 & 5 & 6 & 7 & 8 & 9 & 10 & 11 & 12 & 13 & 14 & 15 \\
\hline $\mathrm{Ca}$ & 16.29 & 13.54 & 13.88 & 16.42 & 14.12 & 14.71 & 16.00 & 15.84 & 13.30 & 17.05 & 16.53 & 15.86 & 16.00 & 16.15 & 15.86 \\
$\mathrm{Co}$ & 19.30 & 17.06 & 16.95 & 20.40 & 16.51 & 16.37 & 20.87 & 17.72 & 15.99 & 20.95 & 18.35 & 18.38 & 20.84 & 19.43 & 18.38 \\
$\mathrm{O}$ & 64.42 & 68.55 & 69.17 & 63.19 & 68.85 & 68.92 & 63.13 & 66.16 & 70.71 & 62.01 & 62.63 & 65.77 & 63.16 & 64.15 & 65.77 \\
$\mathrm{~K}$ & - & 0.84 & - & - & 0.51 & - & - & 0.28 & - & - & 0.48 & - & - & 0.28 & - \\
\hline
\end{tabular}

The singular conditions that make the $\mathrm{K}^{+}$segregate towards the grain boundary is related due to its large difference in size between those of $\mathrm{Ca}^{2+}$ and $\mathrm{Co}^{2+} / \mathrm{Co}^{3+}$, whose ionic radius is $152 \mathrm{pm}$, $114 \mathrm{pm}$, and $\sim 80 \mathrm{pm}$, for $\mathrm{K}^{+}, \mathrm{Ca}^{2+}$ and $\mathrm{Co}^{2+} / \mathrm{Co}^{3+}$ respectively ${ }^{[28]}$. Hence, the large ionic radius size of $\mathrm{K}^{+}$avoids itself from being integrated directly into the $\mathrm{Ca}_{3} \mathrm{Co}_{4} \mathrm{O}_{9}$ lattice. Nevertheless, this same condition that preclude from being integrated into the lattice benefits the $\mathrm{K}^{+}$incorporation to the grain boundary.

The electrical resistivity $\rho$, absolute Seebeck coefficient $S$, and power factor $\left(S^{2} / \rho\right)$ for the pellets with different $\mathrm{K}^{+}$concentrations levels as function of temperature are shown in Figure 5-4. For concentrations below $\mathrm{Ca}_{3} \mathrm{Co}_{4} \mathrm{O}_{9} \mathrm{~K}_{0.10}$, the predominant behavior of the $\rho$ - $\mathrm{T}$ curves are metalliclike behavior, $\mathrm{K}^{+}$samples show a small semiconductor-like tendency around $463 \mathrm{~K}$ and $513 \mathrm{~K}$ for $\mathrm{Ca}_{3} \mathrm{Co}_{4} \mathrm{O}_{9} \mathrm{~K}_{0.05}$ and $\mathrm{Ca}_{3} \mathrm{Co}_{4} \mathrm{O}_{9} \mathrm{~K}_{0.10}$, respectively. Furthermore, the $\mathrm{Ca}_{3} \mathrm{Co}_{4} \mathrm{O}_{9} \mathrm{~K}_{0.15}$ samples exhibit a metallic-semiconductor transition behavior after $513 \mathrm{~K} . \rho$ decreases with the increasing doping concentration of $\mathrm{K}^{+}$up to $x=0.10$. At $323 \mathrm{~K}, \mathrm{Ca}_{3} \mathrm{Co}_{4} \mathrm{O}_{9} \mathrm{~K}_{0.10}$ sample has a value of $\rho \sim 27.6 \mu \Omega \mathrm{m}$ leading to $38 \%$ reduction in relation to the baseline calcium cobaltite. Since the monoclinic structure of the $\mathrm{Ca}_{3} \mathrm{Co}_{4} \mathrm{O}_{9}$ is set up of two misfit layered subsystems: the rock salt-type $\mathrm{Ca}_{2} \mathrm{CoO}_{3}$ layer serving as phonon scattering region to reduce the thermal conductivity and the $\mathrm{CdI}_{2}$-type $\mathrm{CoO}_{2}$ layer serving as electronic transport system to reduce the electrical resistivity. Thus having a more aligned crystal structure could enhanced the electrical properties. 

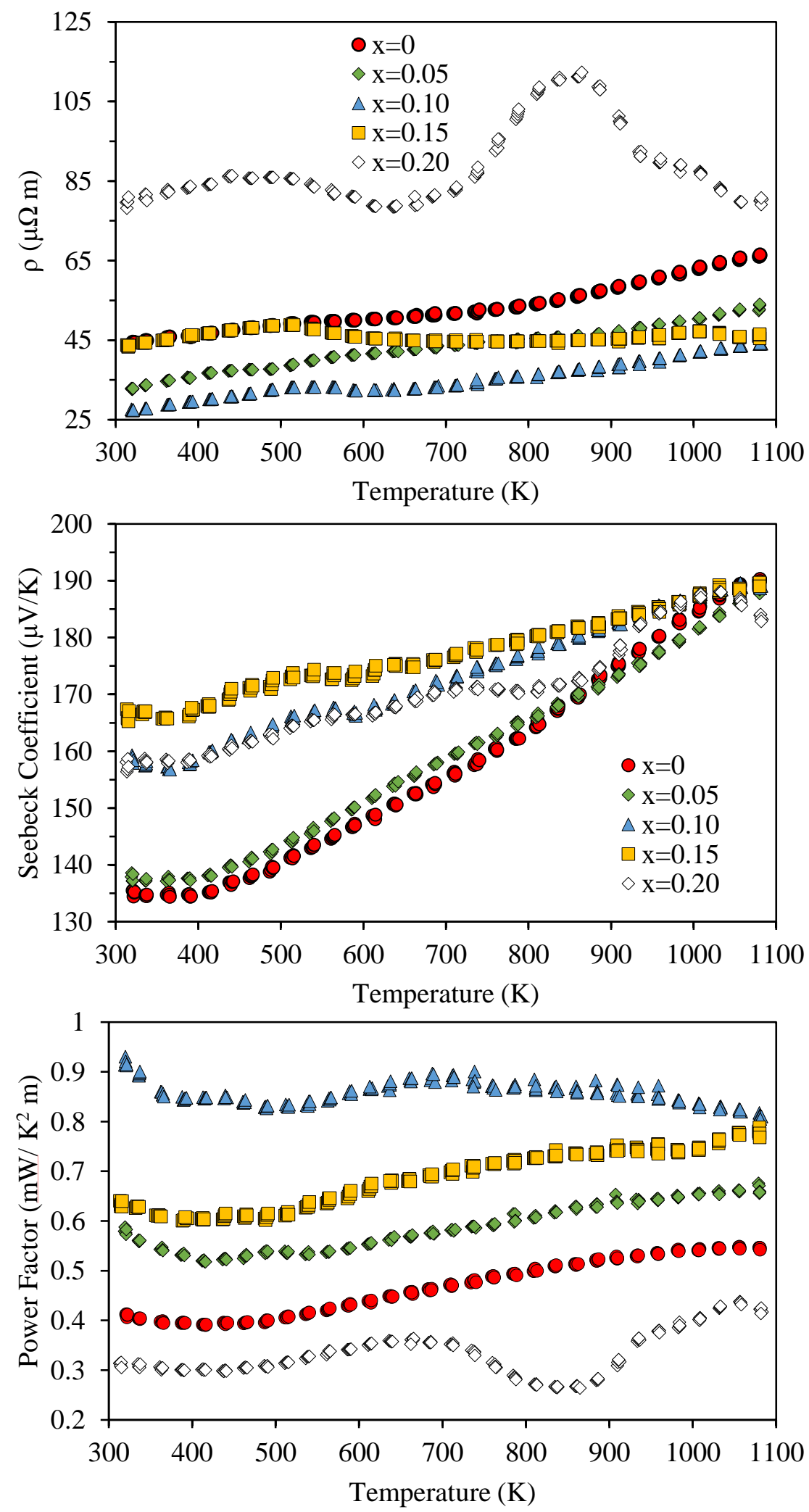

Figure 5-4: Temperature dependence of electrical properties for $\mathrm{Ca}_{3} \mathrm{Co}_{4} \mathrm{O}_{9} \mathrm{~K}_{\mathrm{x}}(\mathrm{x}=0,0.05,0.1$, 0.15 , and 0.2 ). Electrical resistivity (a), Seebeck coefficient (b), and power factor (c). 
Nevertheless, for the increasing doping concentration above $x=0.15$ the electrical resistivity increases due to the more random oriented crystal grains. Additionally, the development of strong textured and aligned crystal on the doped samples $\mathrm{Ca}_{3} \mathrm{Co}_{4} \mathrm{O}_{9} \mathrm{~K}_{x}$ is linked with the carrier mobility improvement ${ }^{[27]}$. Since the electrical conductivity $\sigma$ satisfies $\sigma=n e \mu$, where $\mu$ is the carrier mobility, $e$ is the elementary positive charge constant, and $n$ is the number density of electrons, thus, the decrease of $\rho$ in the bulk material is a straight consequence from a considerably improvement of bulk carrier mobility.

The $\mathrm{S}$ values increase with increasing doping concentrations resulting in an improvement at $323 \mathrm{~K}$ from $\sim 134.5 \mu \mathrm{VK}^{-1}$ to $\sim 167.5 \mu \mathrm{VK}^{-1}$ for $\mathrm{Ca}_{3} \mathrm{Co}_{4} \mathrm{O}_{9}$ and $\mathrm{Ca}_{3} \mathrm{Co}_{4} \mathrm{O}_{9} \mathrm{~K}_{0.15}$, respectively. Most notably, the substantial increase in the $\mathrm{S}$ values from the doped samples are related with a decrease in the carrier concentration of the samples. It is reported that the addition of barrier layers in bulk semiconductor act as an carrier energy filtering mechanism originated by a band offset among the grain boundary and the grain interior which could improve the Seebeck coefficient and the TE power factor in comparison with that from the bulk materials ${ }^{[29]}$. Thus, the disruptive effect of $\mathrm{K}^{+}$ functioning as a barrier layer in the $\mathrm{Ca}_{3} \mathrm{Co}_{4} \mathrm{O}_{9}$ grain boundary block the carrier motion and carrier transport through the adjacent crystals. For that reason, carrier motion across the grain boundary only occurs to those carriers with sufficiently high energy while other low energy carriers are filtered and not contributing to the conduction, leading to a reduced carrier concentration. As a result, the selective scatter of low-energy carriers drastically improved the Seebeck coefficient. However, there is an optimal extent in which carrier filtering benefits the TE power factor, since exceeding the optimal level of carrier filtering could lead to additional loss of carrier mobility due electron scattering at the interface of the crystals ${ }^{[30,31]}$, consequently a loss in carrier mobility will prompt an increase of $\rho$ of the samples, such is the case of the doped $\mathrm{Ca}_{3} \mathrm{Co}_{4} \mathrm{O}_{9} \mathrm{~K}_{0.2}$ sample. In 
addition, $\mathrm{S}$ values show that over-adding $\mathrm{K}^{+}$to the samples does not further decrease the carrier concentration anymore, demonstrating there is a saturation limit on the $\mathrm{K}^{+}$being segregated to the grain boundary.
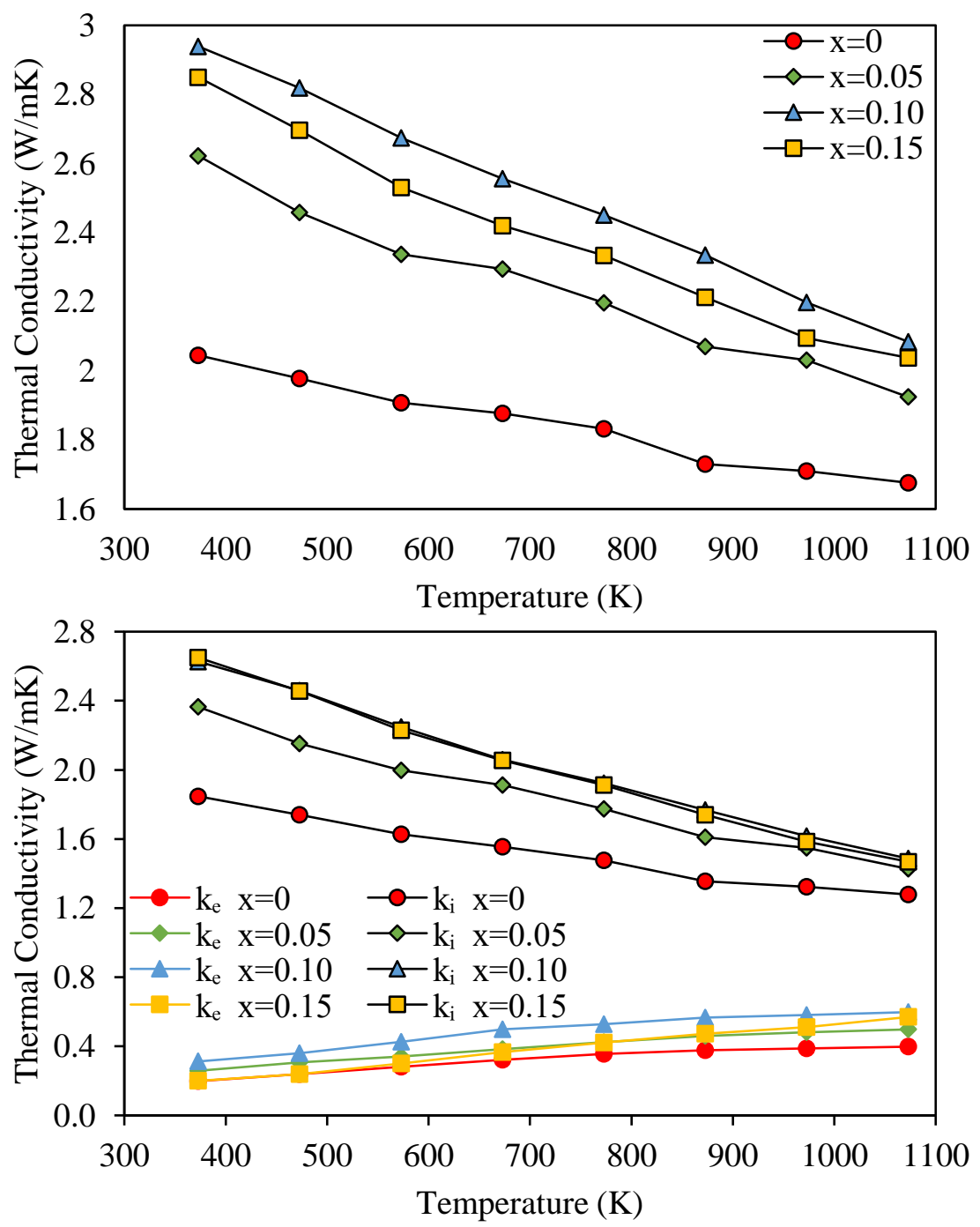

Figure 5-5: Temperature dependence of $(a)$ thermal conductivity $(\kappa-T)$, and $(b)$ electronic and lattice thermal contribution for $\mathrm{Ca}_{3} \mathrm{Co}_{4} \mathrm{O}_{9} \mathrm{~K}_{x}$.

The nonstoichiometric addition of $\mathrm{K}$ increases considerably the thermal conductivity in the material. The mass difference between the host atoms and the dopants in the material play an important role in the scattering of short-wavelength phonons. However, due to the light atomic mass of $\mathrm{K}$, its addition into the $\mathrm{Ca}_{3} \mathrm{Co}_{4} \mathrm{O}_{9} \mathrm{~K}_{\mathrm{x}}$ has a negative impact on both $\kappa_{i}$ and $\kappa_{e}$, corresponding 
to the lattice and electronic contribution to the overall thermal conductivity, respectively. The incorporation of a second dopant could improve the performance of the thermal conductivity.

Although a high electrical power factor was obtained by the $\mathrm{Ca}_{3} \mathrm{Co}_{4} \mathrm{O}_{9} \mathrm{~K}_{\mathrm{x}}$ samples, the ZT of the best sample was improved to a value of 0.42 which correspond to an improvement of only $20 \%$ compared to the pristine $\mathrm{Ca}_{3} \mathrm{Co}_{4} \mathrm{O}_{9}$. The limitation of $\mathrm{K}$ as a dopant resides in the light atomic mass of the element, such inherent characteristic makes difficult to improve the thermal conductivity in the material.

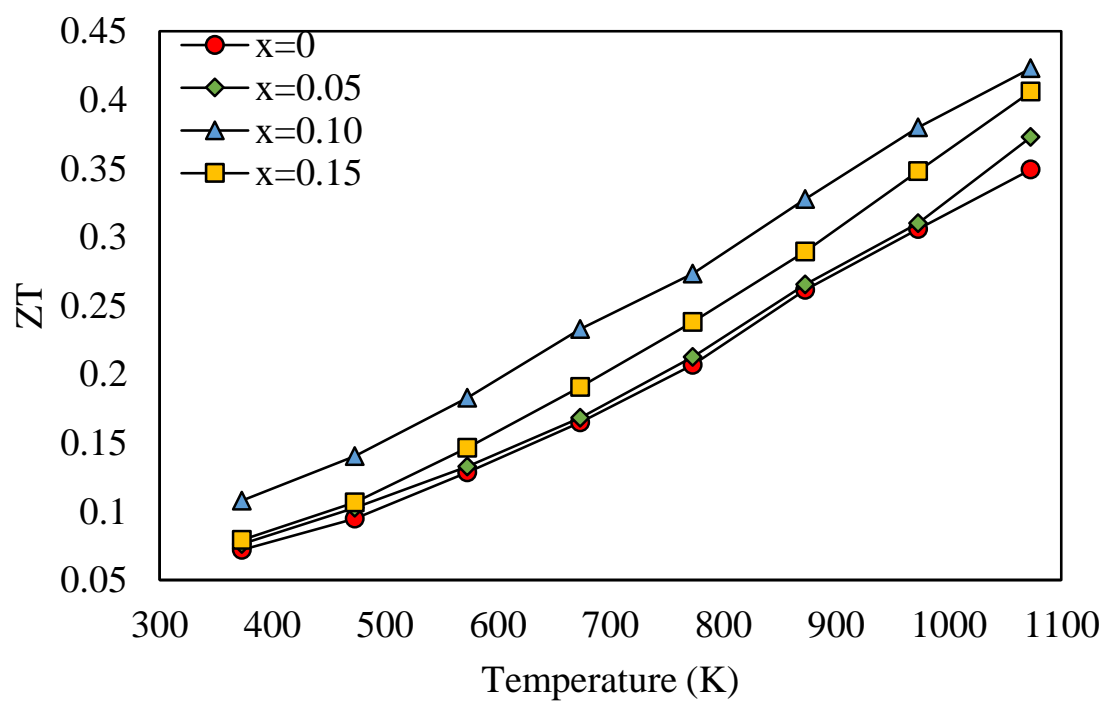

Figure 5-6: Temperature dependence of $\mathrm{ZT}$ for $\mathrm{Ca}_{3} \mathrm{Co}_{4} \mathrm{O}_{9} \mathrm{~K}_{x}$ samples.

\section{5 Conclusion}

Overall, the enhancement of TE performance of polycrystalline $\mathrm{Ca}_{3} \mathrm{Co}_{4} \mathrm{O}_{9}$ by the nonstoichiometric addition $\mathrm{K}^{+}$was shown. Optimal doping chemistry of $\mathrm{Ca}_{3} \mathrm{Co}_{4} \mathrm{O}_{9} \mathrm{~K}_{0.1}$ resulted in the highest TE power factor $930 \mu \mathrm{WK}^{-2} \mathrm{~m}^{-1}$ at $320 \mathrm{~K}$ which is 2.25 times higher than that of pristine $\mathrm{Ca}_{3} \mathrm{Co}_{4} \mathrm{O}_{9}$, whereas at $1073 \mathrm{~K}$ the performance is 1.5 times higher than baseline values. The nanostructure examinations reveal that the crystal lattice was not changed, thus, no substitution at $\mathrm{Ca}^{2+}$ and $\mathrm{Co}^{2+} / \mathrm{Co}^{3+}$ sites exist, rather $\mathrm{K}^{+}$is segregated at the $\mathrm{Ca}_{3} \mathrm{Co}_{4} \mathrm{O}_{9}$ grain boundary. 
Segregation of $\mathrm{K}^{+}$is mainly attributed to the large difference in ionic size in comparison with the $\mathrm{Ca}_{3} \mathrm{Co}_{4} \mathrm{O}_{9}$ lattice sites. The addition of $\mathrm{K}^{+}$acts as a barrier layer in the $\mathrm{Ca}_{3} \mathrm{Co}_{4} \mathrm{O}_{9}$ grain boundary block the carrier motion and carrier transport through the adjacent crystals leading to a reduced carrier concentration and subsequently increase of the $S$ to a value of $167 \mu \mathrm{VK}^{-1}$. Grain boundary segregation also plays a significant role promoting crystal texture and grain alignment and thus increasing carrier mobility in $\mathrm{Ca}_{3} \mathrm{Co}_{4} \mathrm{O}_{9}$, which decreased $\rho$ to a value of $27.6 \mu \Omega \mathrm{m}$. The optimal doping concentration simultaneously decreased the $\rho$ a $38 \%$ value and increase the $S$ a $15 \%$. Such properties enlargement lead to the improved power factor by a $50 \%$. Whereas the thermal conductivity for the best electrical performing material increases negatively to a value $\sim 2.1 \mathrm{~W} / \mathrm{mK}$ which impacts $25 \%$ de thermal properties. The optimal chemistry $\mathrm{Ca}_{3} \mathrm{Co}_{4} \mathrm{O}_{9} \mathrm{~K}_{0.1}$ achieved a peak ZT of 0.42 which represents a value with a $20 \%$ improvement compared to the pristine material. 


\section{References}

1. Stobart, R., M.A. Wijewardane, and Z. Yang, Comprehensive analysis of thermoelectric generation systems for automotive applications. Applied Thermal Engineering, 2017. 112: p. 1433-1444.

2. Kishita, Y., et al., Evaluating the life cycle CO2 emissions and costs of thermoelectric generators for passenger automobiles: a scenario analysis. Journal of Cleaner Production, 2016. 126: p. 607-619.

3. Børset, M.T., et al., Exploring the potential for waste heat recovery during metal casting with thermoelectric generators: On-site experiments and mathematical modeling. Energy, 2017. 118: p. 865-875.

4. Luo, Q., et al., A Thermoelectric Waste-Heat-Recovery System for Portland Cement Rotary Kilns. Journal of Electronic Materials, 2015. 44(6): p. 1750-1762.

5. Gabbar, H.A., et al., Evaluation and optimization of thermoelectric generator network for waste heat utilization in nuclear power plants and non-nuclear energy applications. Annals of Nuclear Energy, 2017. 101: p. 454-464.

6. Bell, L.E., Cooling, Heating, Generating Power, and Recovering Waste Heat with Thermoelectric Systems. Science, 2008. 321(5895): p. 1457.

7. Fergus, J.W., Oxide materials for high temperature thermoelectric energy conversion. Journal of the European Ceramic Society, 2012. 32(3): p. 525-540.

8. Snyder, G.J. and E.S. Toberer, Complex thermoelectric materials. Nature Materials, 2008. 7: p. 105.

9. Lu, H., L. Price, and Q. Zhang, Capturing the invisible resource: Analysis of waste heat potential in Chinese industry. Applied Energy, 2016. 161: p. 497-511.

10. Koumoto, K., et al., Thermoelectric Ceramics for Energy Harvesting. Journal of the American Ceramic Society, 2013. 96(1): p. 1-23.

11. Poudel, B., et al., High-Thermoelectric Performance of Nanostructured Bismuth Antimony Telluride Bulk Alloys. Science, 2008. 320(5876): p. 634.

12. Brostow, W., et al., Bismuth telluride-based thermoelectric materials: Coatings as protection against thermal cycling effects. Journal of Materials Research, 2012. 27(22): p. 2930-2936.

13. Song, X., et al., Significant enhancement of electrical transport properties of thermoelectric $\mathrm{Ca}_{3} \mathrm{Co}_{4} \mathrm{O}_{9+\delta}$ through $\mathrm{Yb}$ doping. Solid State Communications, 2012. 152(16): p. 1509-1512.

14. Chen, S., et al., Effect of precursor calcination temperature on the microstructure and thermoelectric properties of $\mathrm{Ca}_{3} \mathrm{Co}_{4} \mathrm{O}_{9}$ ceramics. Journal of Sol-Gel Science and Technology, 2012. 64(3): p. 627-636.

15. Boyle, C., et al., Grain boundary segregation and thermoelectric performance enhancement of bismuth doped calcium cobaltite. Journal of the European Ceramic Society, 2016. 36(3): p. 601-607.

16. Van Nong, N., et al., Enhancement of the Thermoelectric Performance of p-Type Layered Oxide $\mathrm{Ca}_{3} \mathrm{Co}_{4} \mathrm{O}_{9+\delta}$ Through Heavy Doping and Metallic Nanoinclusions. Advanced Materials, 2011. 23(21): p. 2484-2490.

17. Lim, C.-H., S.-M. Choi, and W.-S. Seo, High-temperature thermoelectric properties of the $\mathrm{Ca}_{3-x} \mathrm{~K}_{x} \mathrm{Co}_{4} \mathrm{O}_{9}(0 \leq x \leq 0.3)$ system. Journal of the Korean Physical Society, 2010. 57(41): p. $1054-1058$. 
18. Tahashi, M., et al., Sintering Temperature Dependence of Thermoelectric Performance and Crystal Phase of Calcium Cobalt Oxides. Journal of the American Ceramic Society, 2010. 93(10): p. 3046-3048.

19. Noudem, J.G., R. Retoux, and S. Lanfredi, Spark plasma texturing (SPT) of p-type $\left[\mathrm{Ca}_{2} \mathrm{CoO}_{3}\right]_{0.62}\left[\mathrm{CoO}_{2}\right]$ thermoelectric oxide. Journal of Alloys and Compounds, 2016. 676: p. 499-504.

20. $\mathrm{Xu}, \mathrm{Y}$. , et al., Influence of external magnetic field on microstructure and electrical transport properties of $\mathrm{Ca}_{3-x} \mathrm{La}_{x} \mathrm{Co}_{4} \mathrm{O}_{9+\delta}$ ceramics. Ceramics International, 2016. 42(9): p. 11404-11410.

21. Woermann, E. and A. Muan, Phase equilibria in the system CaO-cobalt oxide in air. Journal of Inorganic and Nuclear Chemistry, 1970. 32(5): p. 1455-1459.

22. Sotelo, A., et al., Improvement of thermoelectric properties of $\mathrm{Ca}_{3} \mathrm{Co}_{4} \mathrm{O}_{9}$ using soft chemistry synthetic methods. Journal of the European Ceramic Society, 2012. 32(10): p. 2415-2422.

23. Lin, Y.-H., et al., High-Temperature Electrical Transport and Thermoelectric Power of Partially Substituted $\mathrm{Ca}_{3} \mathrm{Co}_{4} \mathrm{O}_{9}$-Based Ceramics. Journal of the American Ceramic Society, 2007. 90(1): p. 132-136.

24. Wang, Y., et al., Nb-doped grain boundary induced thermoelectric power factor enhancement in La-doped $\mathrm{SrTiO}_{3}$ nanoceramics. Journal of Power Sources, 2013. 241: p. 255-258.

25. Farahi, N., et al., Effect of Silicon Carbide Nanoparticles on the Grain Boundary Segregation and Thermoelectric Properties of Bismuth Doped $\mathrm{Mg}_{2} \mathrm{Si}_{0.7} \mathrm{Ge}_{0.3}$. Journal of Electronic Materials, 2016. 45(12): p. 6052-6058.

26. Mehdizadeh Dehkordi, A., et al., Large Thermoelectric Power Factor in Pr-Doped $\mathrm{SrTiO}_{3-\delta}$ Ceramics via Grain-Boundary-Induced Mobility Enhancement. Chemistry of Materials, 2014. 26(7): p. 2478-2485.

27. Carvillo, P., et al., Thermoelectric Performance Enhancement of Calcium Cobaltite through Barium Grain Boundary Segregation. Inorganic Chemistry, 2015. 54(18): p. 90279032.

28. Shannon, R.D., Revised effective ionic radii and systematic studies of interatomic distances in halides and chalcogenides. Acta Crystallographica Section A, 1976. 32(5): p. 751-767.

29. Zide, J.M.O., et al., Demonstration of electron filtering to increase the Seebeck coefficient in In ${ }_{0.53} G a_{0.47} A s / n_{0.53} G a_{0.28} A l_{0.19} A s$ superlattices. Physical Review B, 2006. 74(20): p. 205335.

30. Pichanusakorn, P. and P. Bandaru, Nanostructured thermoelectrics. Materials Science and Engineering: R: Reports, 2010. 67(2): p. 19-63.

31. Zhang, Y., et al., Hot Carrier Filtering in Solution Processed Heterostructures: A Paradigm for Improving Thermoelectric Efficiency. Advanced Materials, 2014. 26(17): p. 2755-2761. 


\section{Chapter 6. Influence of the Dual Non-Stoichiometric Addition of Bismuth and Potassium in Thermoelectric Performance of Calcium Cobaltite Ceramics \\ 6. 1 Complex interactions of dual dopants at GB leading to improved ZT}

The present work describes the effect of a novel approach using the double non-stoichiometric addition of trivalent Bismuth $\left(\mathrm{Bi}^{3+}\right)$ and isovalent Potassium $\left(\mathrm{K}^{+}\right)$to improve the microstructure and thermoelectric performance of misfit layered calcium cobaltite $\mathrm{Ca}_{3} \mathrm{Co}_{4} \mathrm{O}_{9}$ ceramics. The designed nominal chemistry of the polycrystalline ceramic samples in the present study are the $\mathrm{Ca}_{3} \mathrm{Co}_{4} \mathrm{O}_{9}$ and $\mathrm{Ca}_{3} \mathrm{Co}_{4} \mathrm{O}_{9} \mathrm{Bi}_{x} \mathrm{~K}_{y}(x=0.15, \mathrm{y}=0|x=0, \mathrm{y}=0.05| x=0.10, \mathrm{y}=0.05|x=0.125, \mathrm{y}=0.05|$ $x=0.15, \mathrm{y}=0.05 \mid x=0.20, \mathrm{y}=0.05)$. For the dual non-stoichiometric addition system, there are complex interactions between the different dopants that are added to the CCO ceramics simultaneously. Their interactions affected both the electrical power factor and the thermal conductivity, due to the nature and level of the dopants. The incorporation of both $\mathrm{Bi}$ and $\mathrm{K}$ nonstoichiometric addition significantly enhance the crystal texture and grain alignment of the micron sized crystal grains. The nanostructure and chemistry analysis explored under TEM revealed that Bi depletion takes place at the $\mathrm{GBs}$ of the $\mathrm{Ca}_{3} \mathrm{Co}_{4} \mathrm{O}_{9}$ while the lattice shows a greater presence of $\mathrm{Bi}$ with the dual non-stoichiometric approach. Nonetheless, oversized $\mathrm{K}$ is believed to exist segregated at the Grain Boundaries $(\mathrm{GBs})$ of $\mathrm{Ca}_{3} \mathrm{Co}_{4} \mathrm{O}_{9}$ grains. Additionally, XRD analysis provided evidence of the $\mathrm{Ca}_{3} \mathrm{Co}_{4} \mathrm{O}_{9}$ polycrystalline phase for the dual non-stoichiometric addition samples. A substantial increase in the electrical power factor (PF) is obtained for the best performing dual doped sample is about $0.77 \mathrm{mWm}^{-1} \mathrm{~K}^{-2}$ at $1073 \mathrm{~K}$, which represent an increase of $40 \%$ when compared to that of the baseline $\mathrm{Ca}_{3} \mathrm{Co}_{4} \mathrm{O}_{9}$. The incorporation of the heavy atomic mass of $\mathrm{Bi}$ in the dual non-stoichiometric addition offset the rise in thermal conductivity by the addition of K. Overall, the best performing calcium cobaltite is significantly enhanced by the dual doping addition of $\mathrm{Bi}$ and $\mathrm{K}$ resulting in a figure of merit $(Z T)$ of 0.44 at $1073 \mathrm{~K}$ achieved by 
$\mathrm{Ca}_{3} \mathrm{Co}_{4} \mathrm{O}_{9} \mathrm{Bi}_{0.15} \mathrm{~K}_{0.05}$, which is a $30 \%$ increase to the already good $\mathrm{ZT}$ value of 0.35 for the pristine $\mathrm{Ca}_{3} \mathrm{Co}_{4} \mathrm{O}_{9}$. The significant pathway of the dual non-stoichiometric addition on the calcium cobaltite and its impact on the increase in $Z T$ are further discussed in this paper.

\section{2 Introduction}

The demand for energy has continuously risen due to the ever-increasing population and driven by economic development nations. However, as a consequence of this energy demand the pollution has increased and environment is at risk due to fossil-fuel being used as primary source for this energy. It is therefore essential to accelerated the implementation of clean-energy technologies at commercial scale to mitigate the climate change ${ }^{[1]}$ Development of new materials is an important pathway to the evolution towards a more sustainable future based on clean energy generation. ${ }^{[2]}$

Thermoelectric (TE) materials has gained a lot of attraction due to the excessive amount of energy lost as waste heat. TE modules convert energy wasted as heat directly into electricity and feature no moving parts, maintenance free operation, and extended longevity. ${ }^{[3]}$ Thus, electrical power generation by $\mathrm{TE}$ devices provide a promising alternative for recovering high temperature waste heat. Commercial available p-type TE materials such as $\mathrm{BiTe}^{[4]}$ and $\mathrm{SiGe}^{[5]}$ hold values of ZT 1 (threshold for commercial applications ${ }^{[6]}$ ). Still, conventional TE materials are not extensively used in commercial applications because it mostly uses elements that are scarce (high cost), toxic (not environmentally friendly), and instable at high temperatures (poor performance in air). TE oxide materials offers a capable solution to overcome these limiting conditions. ${ }^{[7]}$ $\mathrm{Ca}_{3} \mathrm{Co}_{4} \mathrm{O}_{9}(\mathrm{CCO})$ has been widely considered as one of the most promising candidates as $p$-type TE material ${ }^{[3]}$ due to its high temperature stability ${ }^{[8]}$ and ZT value of 0.87 for single crystal. ${ }^{[9]}$ Further progress of the polycrystalline form of $\mathrm{CCO}$ must be made owing that it only achieves a 
small fraction of the TE performance of CCO single crystal, with reported $Z T$ values $\sim 0.3$ at high temperature for most polycrystalline $\mathrm{CCO}^{[7]}$.

Nonetheless, the main challenge to maximize the performance and improve the suitability in commercial application in thermoelectric materials is the inherent conflicting material properties of the bulk materials ${ }^{[10]}$. The energy conversion efficiency of the TE materials is described by the $Z T$, which is defined as $Z T=S^{2} / \rho^{-1} \kappa^{-1} T$, where $S, \rho, S^{2} / \rho^{-1}$, and $\kappa$ are the Seebeck coefficient, electrical resistivity, electrical power factor (PF), and thermal conductivity, respectively. $\kappa$ can be expressed as a contribution of the $\kappa_{i}$ (lattice) and $\kappa_{e}$ (electronic) as $\kappa=\kappa_{i}+\kappa_{e}$. Additionally, the $\kappa_{e}$ is directly linked to the electrical conductivity $(\sigma)$ by the Wiedemann-Franz law $-\kappa_{e}=L \sigma T$, where $\mathrm{L}$ is the Lorenz number. Thus, optimization of all these parameters is compromised because all of them depend on the interconnected electronic properties of the material $^{[11]}$ which make it difficult to achieve a high TE efficiency. One approach to overcome these limiting conditions is by modifying the GBs in a material, since the GBs can help to control the microstructure and the material properties and provide opportunities for new material design. ${ }^{[12]}$ Additionally, GBs engineering could also play an important role in mechanical properties for TE materials. ${ }^{[13]}$ Therefore, to maximize the energy conversion efficiency of polycrystalline $\mathrm{CCO}$, and improve the $\mathrm{PF}$ with a simultaneous reduction of $\kappa$ a better understanding of the effects on engineering GBs for the dual non-stoichiometric addition in $\mathrm{CCO}$ is required.

Numerous researchers have reported the important influence on engineering GBs for the TE properties optimization of different materials ${ }^{[4,14-22]}$. Most notably, it has been previously explored the segregation at the GBs of doping elements into the $\mathrm{CCO} \cdot{ }^{[23-26]}$ All of the doping elements investigated are larger than the host ions in the CCO. The previous research proposed that the driving force for the single dopant GBs segregation is mainly influenced by the ionic size effect 
rather than the Coulomb interaction. ${ }^{[23,25,26]}$ Additionally, there is a likewise effect for a dual doping with simultaneous stoichiometric substitution and non-stoichiometric addition where the foremost reason for GBs segregation is associated to the strain effect. The reported dual-doping elements have demonstrated a selective segregation at the GBs, in which bigger over-sized atoms segregate to the GBs, displacing smaller-sized atoms to the interior of the crystal lattice. ${ }^{[24]}$ Furthermost, to our best knowledge there is not previous report on the dual non-stoichiometric addition in $\mathrm{CCO}$. Hence, to further understand the influence of the GBs engineering and their impact on the nanostructure and TE performance of CCO, the dual non-stoichiometric addition of $\mathrm{Bi}$ and oversized $\mathrm{K}$ was investigated. To get the best benefit from the GBs engineering, the polycrystalline ceramic samples were prepared using sol-gel ${ }^{[27]}$, and by previous reported optimal conditions in the calcination ${ }^{[28]}$ and sintering stages ${ }^{[29]}$, to mitigate the possible formation of $\mathrm{Co}_{3} \mathrm{O}_{4}$ which affect the performance of CCO. ${ }^{[30]}$

\section{3 Experimental section}

The polycrystalline ceramic samples $\mathrm{Ca}_{3} \mathrm{Co}_{4} \mathrm{O}_{9}$ and $\mathrm{Ca}_{3} \mathrm{Co}_{4} \mathrm{O}_{9} \mathrm{Bi}_{x} \mathrm{~K}_{y}(x=0.15, \mathrm{y}=0|x=0, \mathrm{y}=0.05|$ $x=0.100, \mathrm{y}=0.05|x=0.125, \mathrm{y}=0.05| x=0.150, \mathrm{y}=0.05 \mid x=0.200, \mathrm{y}=0.05)$ were synthesized by the conventional chemical sol-gel route. The precursor powders were obtained mixing the chemical reagents according to the stoichiometric ratios. $\mathrm{Ca}\left(\mathrm{NO}_{3}\right)_{2} \cdot 4 \mathrm{H}_{2} \mathrm{O}\left(99 \%\right.$, Acros Organics), $\mathrm{Co}\left(\mathrm{NO}_{3}\right)$ ${ }_{2} \cdot 6 \mathrm{H}_{2} \mathrm{O}\left(99 \%\right.$, Acros Organics), $\mathrm{Bi}\left(\mathrm{NO}_{3}\right)_{3} \cdot 5 \mathrm{H}_{2} \mathrm{O}(98 \%$, Acros Organics), and $\mathrm{KNO} 3$ (99\%, Alfa Aesar) were dissolved in deionized water. Citric acid (BDH Chemical), ethylene glycol, and polyethylene glycol were used to polymerize the mixture, while nitric acid was added to the solution to facilitate the sol-gel synthesis, nitrate salts decomposition, and the new compound formation. The mixture was mechanically stirred at $353 \mathrm{~K}$ for $3 \mathrm{~h}$. The obtained sol-gel solution was ashed at $773 \mathrm{~K}$ for $2 \mathrm{hr}$ in air inside a box furnace. The resulting ashes were ball-milled in 
ethyl alcohol in a stage with zirconia balls and then dried at room temperature with a final manually ground stage after that. The fine ashes were calcined at $973 \mathrm{~K}$ for $4 \mathrm{~h}$ in a tube furnace with a constant oxygen flow. The final precursor powders were uniaxially pressed into round pellets under a $1 \mathrm{Gpa}$ pressure at room temperature. The bulk samples were sintered at temperature 1233 $\mathrm{K}$ in a tube furnace with a constant oxygen flow and then cut into rectangular pellets to obtain the final desired sample for either the electrical or thermal measurements. The apparent densities for all bulk samples are listed in Table 6-1.

Table 6-1: Apparent density of the $\mathrm{Ca}_{3} \mathrm{Co}_{4} \mathrm{O}_{9} \mathrm{Bi}_{x} \mathrm{~K}_{y}$ samples.

\begin{tabular}{lll}
\hline \multicolumn{2}{l}{$\mathrm{Ca}_{3} \mathrm{Co}_{4} \mathrm{O}_{9} \mathrm{Bi}_{\mathrm{x}} \mathrm{K}_{\mathrm{y}}$} & Average Apparent density $\left(\mathrm{g} / \mathrm{cm}^{3}\right)$ \\
\hline $\mathrm{x}=0$ & $\mathrm{y}=0$ & 3.883 \\
$\mathrm{x}=0$ & $\mathrm{y}=0.05$ & 4.119 \\
$\mathrm{x}=0.15$ & $\mathrm{y}=0$ & 3.575 \\
$\mathrm{x}=0.10 \quad \mathrm{y}=0.05$ & 4.296 \\
$\mathrm{x}=0.125$ & $\mathrm{y}=0.05$ & 4.359 \\
$\mathrm{x}=0.150 \quad \mathrm{y}=0.05$ & 4.208 \\
$\mathrm{x}=0.200 \mathrm{y}=0.05$ & 4.405 \\
$\mathrm{x}=0.150 \mathrm{y}=0.10$ & 4.449 \\
\hline
\end{tabular}

The electrical resistivity $(\rho)$ and absolute Seebeck coefficient $(S)$ were measured by the fourterminal method using a Linseis LSR-1100 unit. The measurements were performed direction to the pressed direction from $323 \mathrm{~K}$ to $1073 \mathrm{~K}$ under a low pressure He environment. The thermal conductivity was calculated using the equation $\kappa=\lambda C_{p} \rho m$, where $C_{p}, \lambda$, and $m$ are the specific heat, thermal diffusivity, and mass density, respectively. The $C p$ and $\lambda$ values were obtained from 323 K to $1073 \mathrm{~K}$ using a Linseis Laser Flash Analyzer 1200. The measurements were performed perpendicular to the pressed direction under a low-pressure air environment. X-Ray powder Diffraction (XRD) analysis was performed using a PANatycal X'Pert Pro XRD unit for crystal phase and lattice parameter determination purposes. The ground powders from the sintered samples were used in the XRD analysis using $\mathrm{Cu}$ K-alpha radiation, $45 \mathrm{kV}$ tension and $40 \mathrm{~mA}$ 
current at room temperature. The cross-section morphology of the sintered pellets was observed using a Hitachi-S4700F Scanning Electron Microscope (SEM) unit. The samples for transmission electron microscopy (TEM) were prepared by mechanically polishing and ion milling in a liquidnitrogen-cooled holder. Electron diffraction, diffraction contrast, and high-resolution TEM imaging were performed in a JEM-2100 operated at $200 \mathrm{kV}$. The chemical analysis was carried out using energy dispersive spectroscopy (EDS) under TEM.

\section{4 Results}

The influence on the electrical transport properties by the dual non-stoichiometric addition of $\mathrm{K}$ and $\mathrm{Bi}$ are presented in Figure 6-1 by the variation of the $\rho, \mathrm{S}$, and $\mathrm{S}^{2} / \rho$ for the pellets with the different concentrations levels as a function of temperature. The $\rho$-T curves for samples with only one doping element show a metallic-like behavior. Whereas, for the samples with dual doping the predominant behavior of the $\rho$-T curves is semiconductor-like behavior and the $\rho$ slope of the curves is not as pronounce as the temperature increases, except the sample $\mathrm{Ca}_{3} \mathrm{Co}_{4} \mathrm{O}_{9} \mathrm{Bi}_{0.15} \mathrm{~K}_{0.05}$ that shows also a metallic-like behavior. Adding a first dopant such as $\mathrm{K}$ at a doping level $\mathrm{y}=0.05$ slightly decreases the $\rho$ in the broad temperature range. But after adding Bi as second dopant, first there is a small increase in the low temperature regime for $\mathrm{x}=0.10$, and with a doping level up to $y=0.15$ the $\rho$ further decreases. The best performing dual doped concentration reaches the lowest $\rho$ value of $27.7 \mu \Omega \mathrm{m}$ at $315 \mathrm{~K}$ which correspond to a reduction of $39 \%$ in comparison with that of the baseline calcium cobaltite. Further addition of either element Bi or K increases significantly the $\rho$ values of the samples.

The dual non-stoichiometric addition of $\mathrm{Bi}$ and $\mathrm{K}$ into $\mathrm{CCO}$ affect considerably the $S$, as shown in Figure 6-1 (b). K added as only dopant has a small influence on the $S$ at the low temperature regime, whereas $\mathrm{Bi}$ alone has a more comprehensive influence on the high temperature with very 

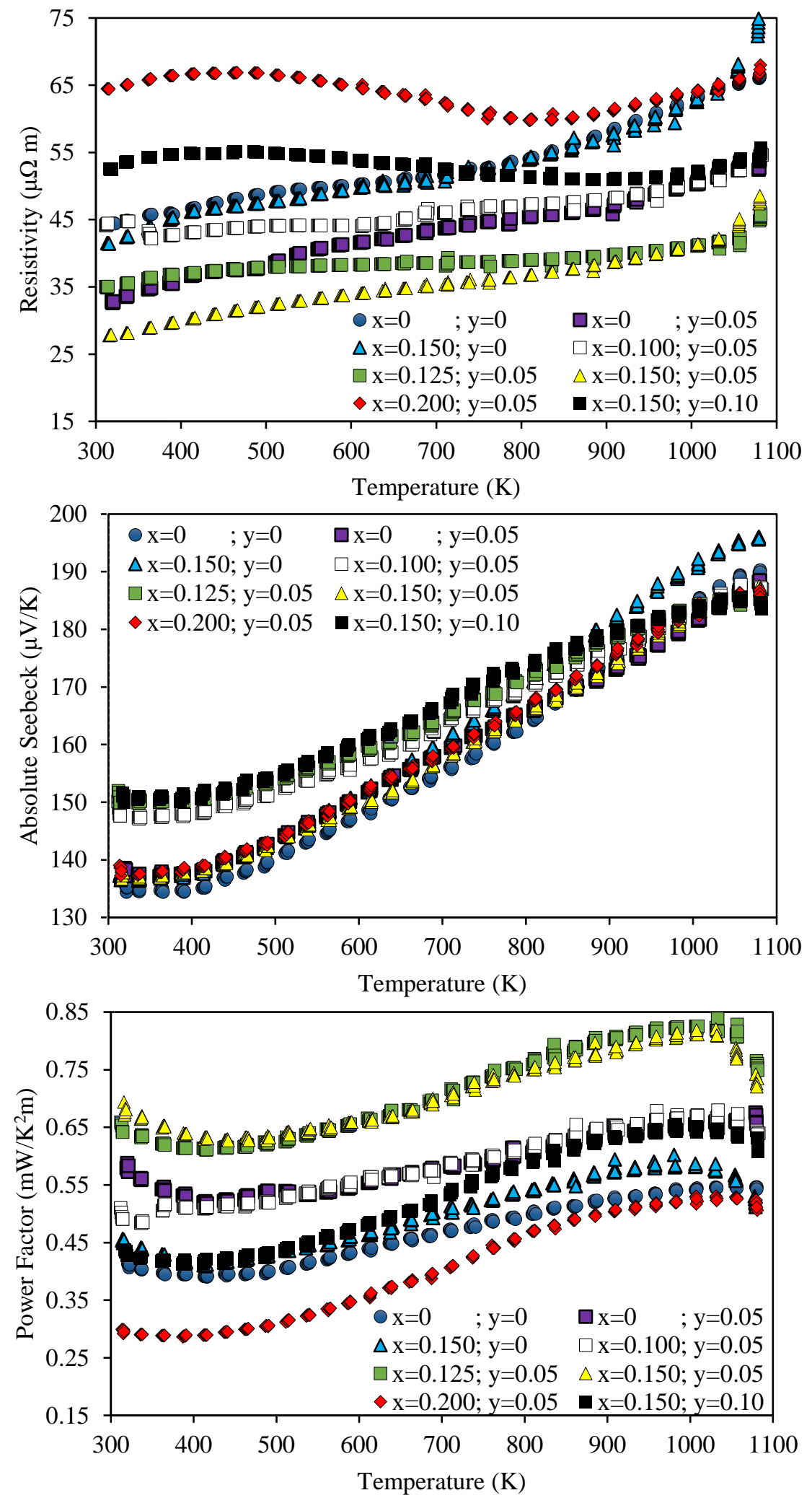

Figure 6-1: Temperature dependence of electrical properties for $\mathrm{Ca}_{3} \mathrm{Co}_{4} \mathrm{O}_{9} \mathrm{Bi}_{x} \mathrm{~K}_{y^{\prime}}$ (a) $\rho$-T, (b) $S-T$, and (c) $S^{2} / \rho-T$. 
slightly improvement at low temperature. Most importantly, with a dual non-stoichiometric addition in a ratio below $0.125 / 0.05$ (including those of $0.10 / 0.05$ and $0.15 / 0.1$ ) for $\mathrm{Bi}$ and $\mathrm{K}$, respectively, the $S$ is greatly enhanced at low temperature to a value $\sim 150 \mu \mathrm{VK}^{-1}$. In contrast, a dual non-stoichiometric addition in a ratio above 0.15/0.05 (including 0.20/0.05) for Bi and $\mathrm{K}$, respectively, do not have any important influence on the $S$, with values $\sim 138 \mu \mathrm{VK}^{-1}$ close to that of the pristine $\mathrm{CCO}$. Additionally, the influence at high temperature of the Bi as only dopant is not conserved with the dual doping approach.

The electrical PF is enhanced to its largest value of $0.82 \mathrm{mWm}^{-1} \mathrm{~K}^{-2}$ (although decreases to 0.77 $\mathrm{mWm}^{-1} \mathrm{~K}^{-2}$ at $1073 \mathrm{~K}$ ) due to the combine influence of the improvements in the $\rho$ value and the enhanced $\mathrm{S}$ for $\mathrm{Ca}_{3} \mathrm{Co}_{4} \mathrm{O}_{9} \mathrm{Bi}_{0.15} \mathrm{~K}_{0.05}$. The improvement corresponds to a $40 \%$ increase compared to that of the pristine $\mathrm{CCO}$. Moreover, $\mathrm{Ca}_{3} \mathrm{Co}_{4} \mathrm{O}_{9} \mathrm{Bi}_{0.125} \mathrm{~K}_{0.05}$ sample has a slightly better PF, however the inclusion of a higher concentration of $\mathrm{Bi}$ is desire, as a higher concentration of heavy elements could scatter phonons better. Increasing the doping level further than $\mathrm{Ca}_{3} \mathrm{Co}_{4} \mathrm{O}_{9} \mathrm{Bi}_{0.15} \mathrm{~K}_{0.05}$ does not further improve the thermoelectric properties, demonstrating there is a limit on the dual non-stoichiometric benefit.

The influence on the thermal transport properties and ZT by the dual non-stoichiometric addition of $\mathrm{K}$ and $\mathrm{Bi}$ are presented in Figure 6-2 by the variation of the $\kappa, \kappa_{i}, \kappa_{e}$ and $Z T$ for the pellets with the different concentrations levels as a function of temperature. Addition of $\mathrm{K}$ alone into the $\mathrm{CCO}$ increases $\kappa$, due to the increase in both the lattice and electronic contribution. Further addition of $\mathrm{Bi}$ as second dopant gradually decreases the $\kappa$ with the increasing doping level up to $\mathrm{x}=0.15$. The reduction of the $\kappa$ in the dual-doped CCO is mainly due to the decline of the lattice contribution. It can be seen that exceeding the $\mathrm{Bi}$ level higher than $\mathrm{Ca}_{3} \mathrm{Co}_{4} \mathrm{O}_{9} \mathrm{Bi}_{0.15} \mathrm{~K}_{0.05}$ increases the lattice contribution, however due to decreased electronic properties the overall $\kappa$ is decreased. 

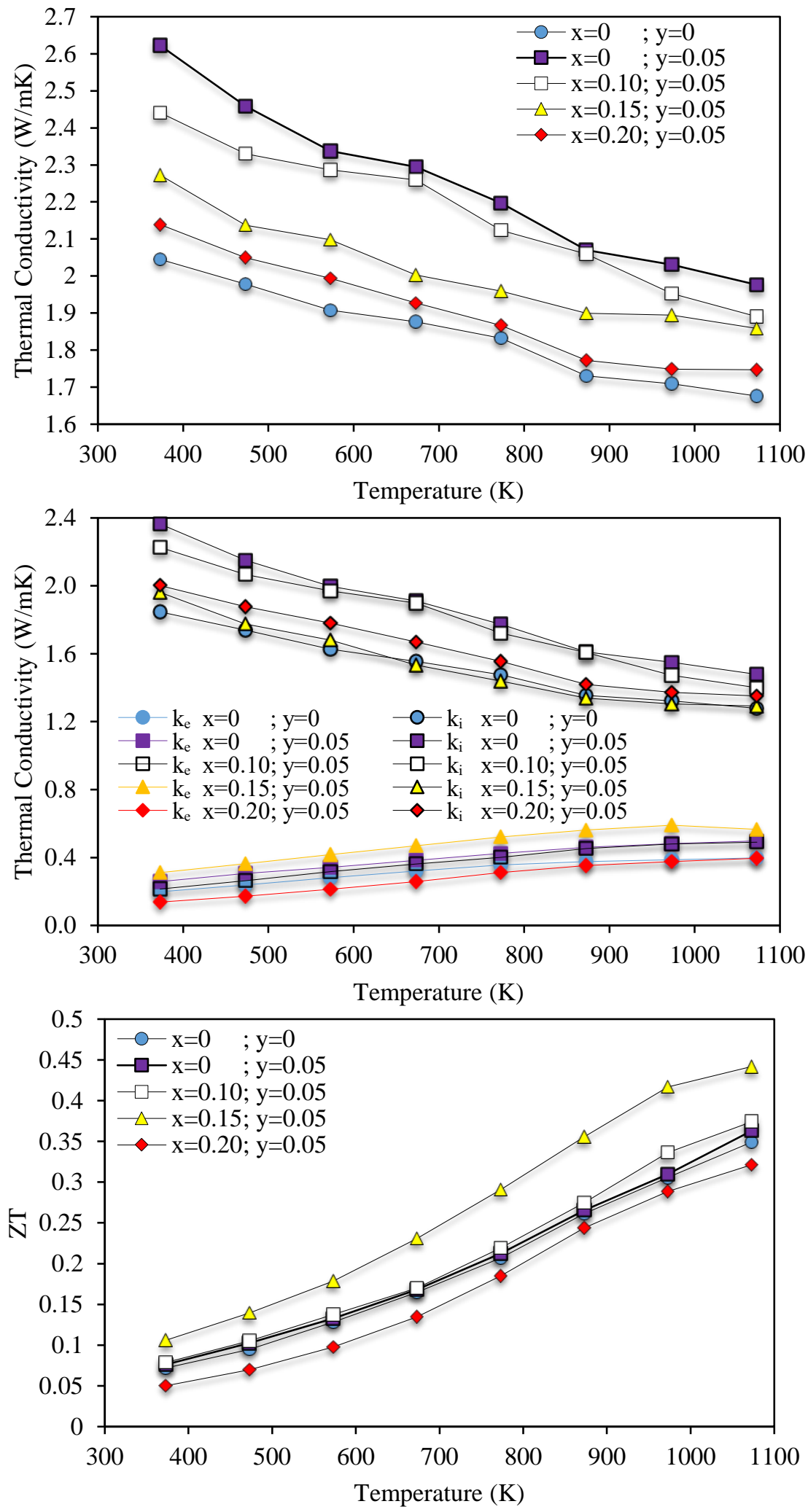

Figure 6-2: Temperature dependence of (a) thermal conductivity ( $\kappa-T)$, (b) electronic and lattice thermal contribution, and (c) thermoelectric figure of merit (ZT) for $\mathrm{Ca}_{3} \mathrm{Co}_{4} \mathrm{O}_{9} \mathrm{Bi}_{x} \mathrm{~K}_{y}$. 
Figure of merit is enhanced by the improved thermal and electronic transport properties in the dual non-stoichiometric addition of Bi and K. Figure 6-2 (c) shows that there is a first improvement by the addition of $\mathrm{K}$ alone. There is a supplementary enlargement of $Z T$ with the further inclusion of $\mathrm{Bi}$ doping level up to $\mathrm{x}=0.15$, reaching a high $Z T$ value of 0.44 at $1073 \mathrm{~K}$ for the $\mathrm{Ca}_{3} \mathrm{Co}_{4} \mathrm{O}_{9} \mathrm{Bi}_{0.15} \mathrm{~K}_{0.05}$ samples. The obtained $\mathrm{ZT}$ value represents a $30 \%$ improvement in comparison to that of the $\mathrm{CCO}$ baseline.

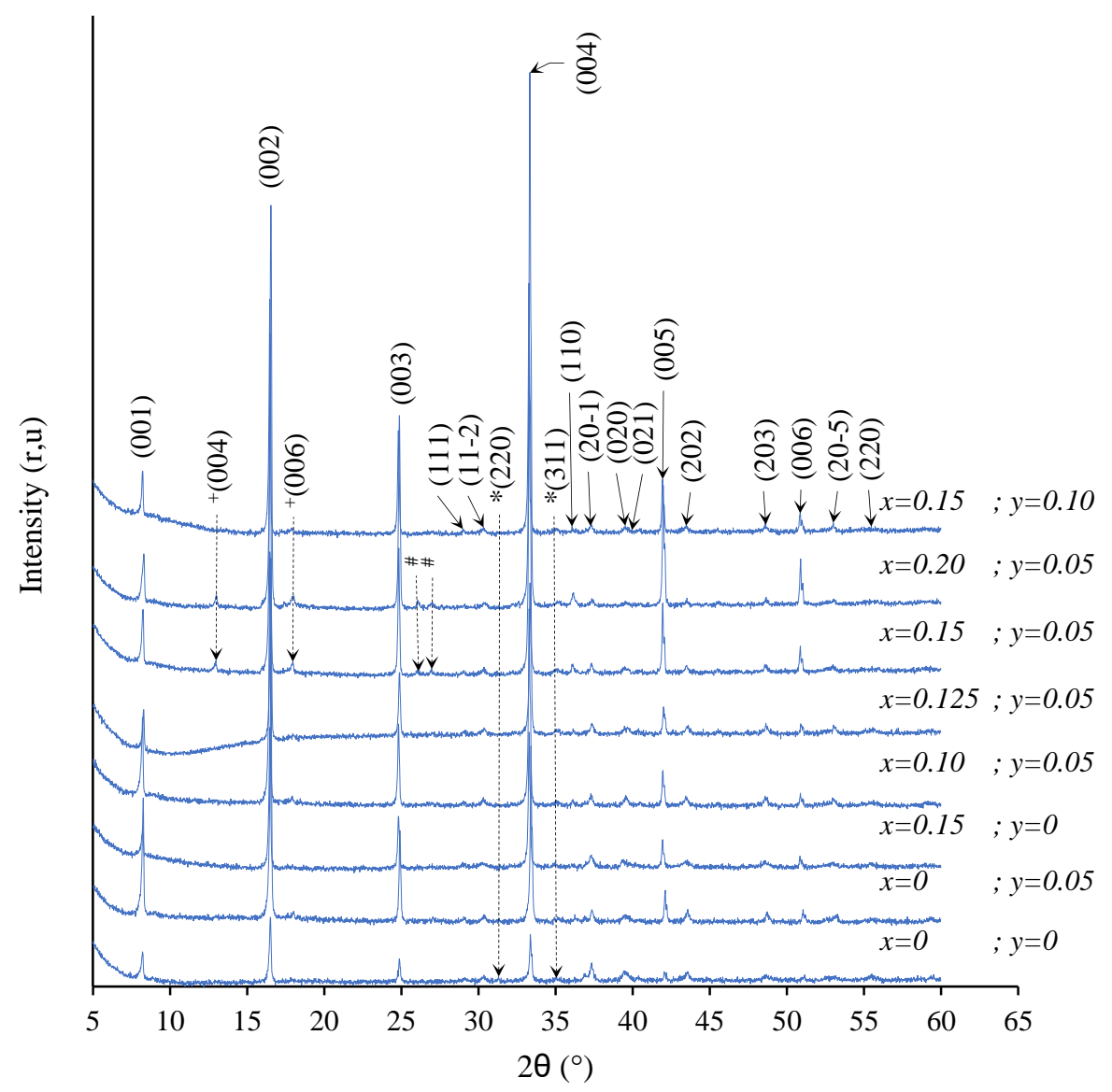

Figure 6-3: $X R D$ powder diffraction patterns for the undoped $\mathrm{Ca}_{3} \mathrm{CO}_{4} \mathrm{O}_{9}$ and the doped $\mathrm{Ca}_{3} \mathrm{Co}_{4} \mathrm{O}_{9} \mathrm{Bi}_{x} \mathrm{~K}_{y}$ samples. In all samples, monoclinic $\mathrm{Ca}_{3} \mathrm{Co}_{4} \mathrm{O}_{9}$ were observed. Nonetheless, a minor amount of $\mathrm{Co}_{3} \mathrm{O}_{4}$ (marked as $\left.{ }^{*}\right), \mathrm{Bi}_{2} \mathrm{Ca}_{2} \mathrm{Co}_{2} \mathrm{O}_{x}$ (marked as ${ }^{+}$), and an unidentified phase (marked as $\left.{ }^{\#}\right)$ were observed.

The XRD patterns of $\mathrm{Ca}_{3} \mathrm{Co}_{4} \mathrm{O}_{9} \mathrm{Bi}_{\mathrm{x}} \mathrm{K}_{\mathrm{y}}$ obtained at room temperature conditions from ground powders of the sintered pellets are shown in Figure 6-3. For visualization purposes, patterns have 
been plotted with a small offset. XRD characteristic peaks obtained from the samples can be indexed as those from the $\mathrm{Ca}_{3} \mathrm{Co}_{4} \mathrm{O}_{9}$ phase with monoclinic symmetry. The patterns exhibit a small shift to a lower Bragg's angle of the peaks for those concentrations with Bi. The shift to a lower Bragg's angle indicates Bi has been incorporated to the crystal and increased the lattice parameter values for $\mathrm{Ca}_{3} \mathrm{Co}_{4} \mathrm{O}_{9}$. There is not significant shift of the XRD peaks with the incorporation of $\mathrm{K}$. The $\mathrm{Co}_{3} \mathrm{O}_{4}$ secondary phase is present in very small amounts in all samples. There is also a very small presence of characteristic diffraction peaks from an unidentified secondary phase and a secondary phase corresponding to $\mathrm{Bi}_{2} \mathrm{Ca}_{2} \mathrm{Co}_{2} \mathrm{O}_{\mathrm{x}}$ for concentrations $\mathrm{Ca}_{3} \mathrm{Co}_{4} \mathrm{O}_{9} \mathrm{Bi}_{0.15} \mathrm{~K}_{0.05}$ and $\mathrm{Ca}_{3} \mathrm{Co}_{4} \mathrm{O}_{9} \mathrm{Bi}_{0.20} \mathrm{~K}_{0.05}$. The calculated lattice parameter values for samples with the different doping level are listed in Table 6-2. With the increase of the Bi doping level, there is an increase in the lattice parameter values $a, b_{1}, c$, and $\beta$, which is accompanied by the decrease in the lattice parameter $b_{2}$. Compared to the effect of $\mathrm{Bi}$, the non-stoichiometric addition of $\mathrm{K}$ does not have an important impact on the lattice parameter values, suggesting that $\mathrm{K}$ is not present in the crystal lattice.

Table 6-2: Lattice parameters $a, b_{1}, b_{2}, c$, and $\beta$ for the $C a_{3} \mathrm{Co}_{4} \mathrm{O}_{9} B i_{x} K_{y}$ samples. $b_{1}$ and $b_{2}$ refer to b-axis lattice parameters in the rock-salt layer $\mathrm{Ca}_{2} \mathrm{CoO}_{3}$ and the $\mathrm{CoO}_{2}$ layer, respectively.

\begin{tabular}{lllllll}
\hline $\mathrm{Ca}_{3} \mathrm{Co}_{4} \mathrm{O}_{9} \mathrm{Bi}_{\mathrm{x}} \mathrm{K}_{\mathrm{y}}$ & $\mathrm{a}(\AA)$ & $\mathrm{b}_{1}(\AA)$ & $\mathrm{b}_{2}(\AA)$ & $\mathrm{c}(\AA)$ & $\beta\left(^{\circ}\right)$ \\
\hline $\mathrm{x}=0 \quad ; \mathrm{y}=0$ & 4.867 & 4.563 & 2.792 & 10.866 & 98.878 \\
$\mathrm{x}=0 \quad ; \mathrm{y}=0.05$ & 4.867 & 4.564 & 2.790 & 10.863 & 99.049 \\
$\mathrm{x}=0.15 \quad ; \mathrm{y}=0$ & 4.883 & 4.566 & 2.780 & 10.907 & 99.208 \\
$\mathrm{x}=0.10 \quad ; \mathrm{y}=0.05$ & 4.880 & 4.564 & 2.789 & 10.898 & 99.048 \\
$\mathrm{x}=0.125 ; \mathrm{y}=0.05$ & 4.894 & 4.565 & 2.783 & 10.898 & 99.331 \\
$\mathrm{x}=0.15 ; \mathrm{y}=0.05$ & 4.894 & 4.565 & 2.771 & 10.905 & 99.357 \\
$\mathrm{x}=0.20 ; \mathrm{y}=0.05$ & 4.911 & 4.565 & 2.762 & 10.909 & 99.729 \\
$\mathrm{x}=0.15 \quad ; \mathrm{y}=0.10$ & 4.892 & 4.565 & 2.777 & 10.908 & 99.196 \\
\hline
\end{tabular}

Figure 6-4 show the top-view SEM images taken from the $\mathrm{Ca}_{3} \mathrm{Co}_{4} \mathrm{O}_{9} \mathrm{Bi}_{x}$ pellets after sintering. The incorporation of both dopants have a positive influence on the development of the crystal 
texture and the grain growth. The micron-sized crystal exhibits a plate-shape morphology. Figure 6-5 shows the cross-sectional SEM images taken from the fractured surface of the $\mathrm{Ca}_{3} \mathrm{Co}_{4} \mathrm{O}_{9} \mathrm{Bi}_{x} \mathrm{~K}_{y}$ pellets after sintering. The arrow in each picture indicates the pressing direction of the pellets. With the incorporation of $\mathrm{K}$ and later with the increase in the $\mathrm{Bi}$ doping level, the size of the crystals gradually increases parallel to the $a-b$ plane of the elongated crystal grains, while remained with a similar size in the $c$-axes. Figure 6-4 and Figure 6-5 clearly depicts that samples present large anisotropy with crystals having the larger dimension in direction to the $a-b$ plane of the monoclinic $\mathrm{Ca}_{3} \mathrm{Co}_{4} \mathrm{O}_{9}$ and the $c$-axis parallel to the pressed direction of the pellets. $\mathrm{Ca}_{3} \mathrm{Co}_{4} \mathrm{O}_{9} \mathrm{Bi}_{0.15} \mathrm{~K}_{0.05}$ sample presents the best texture and preferentially aligned crystal orientation.
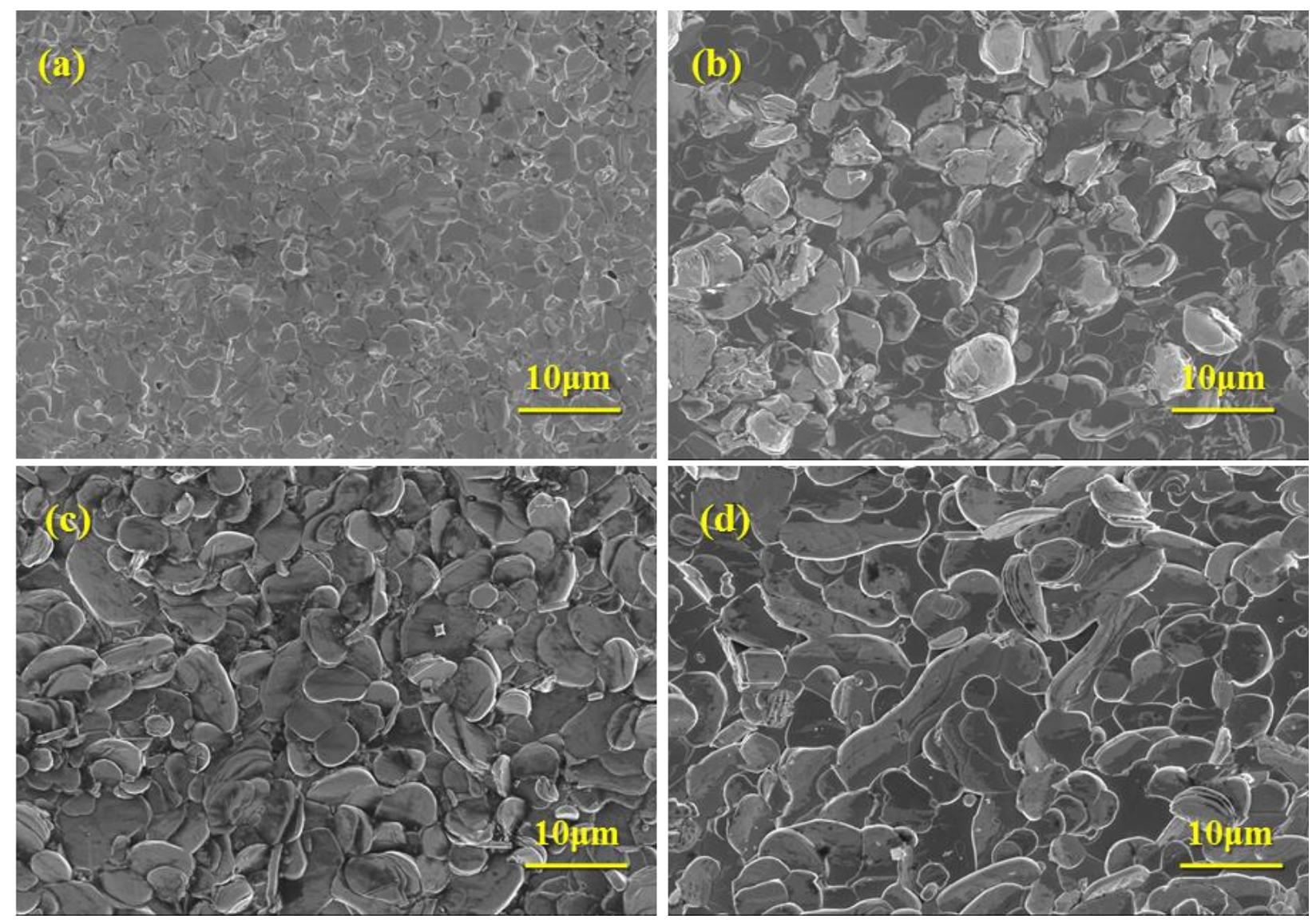

Figure 6-4: $\mathrm{SEM}$ images from the top surface of $\mathrm{Ca}_{3} \mathrm{Co}_{4} \mathrm{O}_{9} \mathrm{Bi}_{x} \mathrm{~K}_{y}$ samples after sintering stage. (a) $\mathrm{Ca}_{3} \mathrm{CO}_{4} \mathrm{O}_{9}$, (b) $\mathrm{Ca}_{3} \mathrm{Co}_{4} \mathrm{O}_{9} \mathrm{~K}_{0.05}$, (c) $\mathrm{Ca}_{3} \mathrm{CO}_{4} \mathrm{O}_{9} \mathrm{~K}_{0.05} \mathrm{Bi}_{0.1}$, and (d) $\mathrm{Ca}_{3} \mathrm{Co}_{4} \mathrm{O}_{9} \mathrm{~K}_{0.05} \mathrm{Bi}_{0.15}$. 

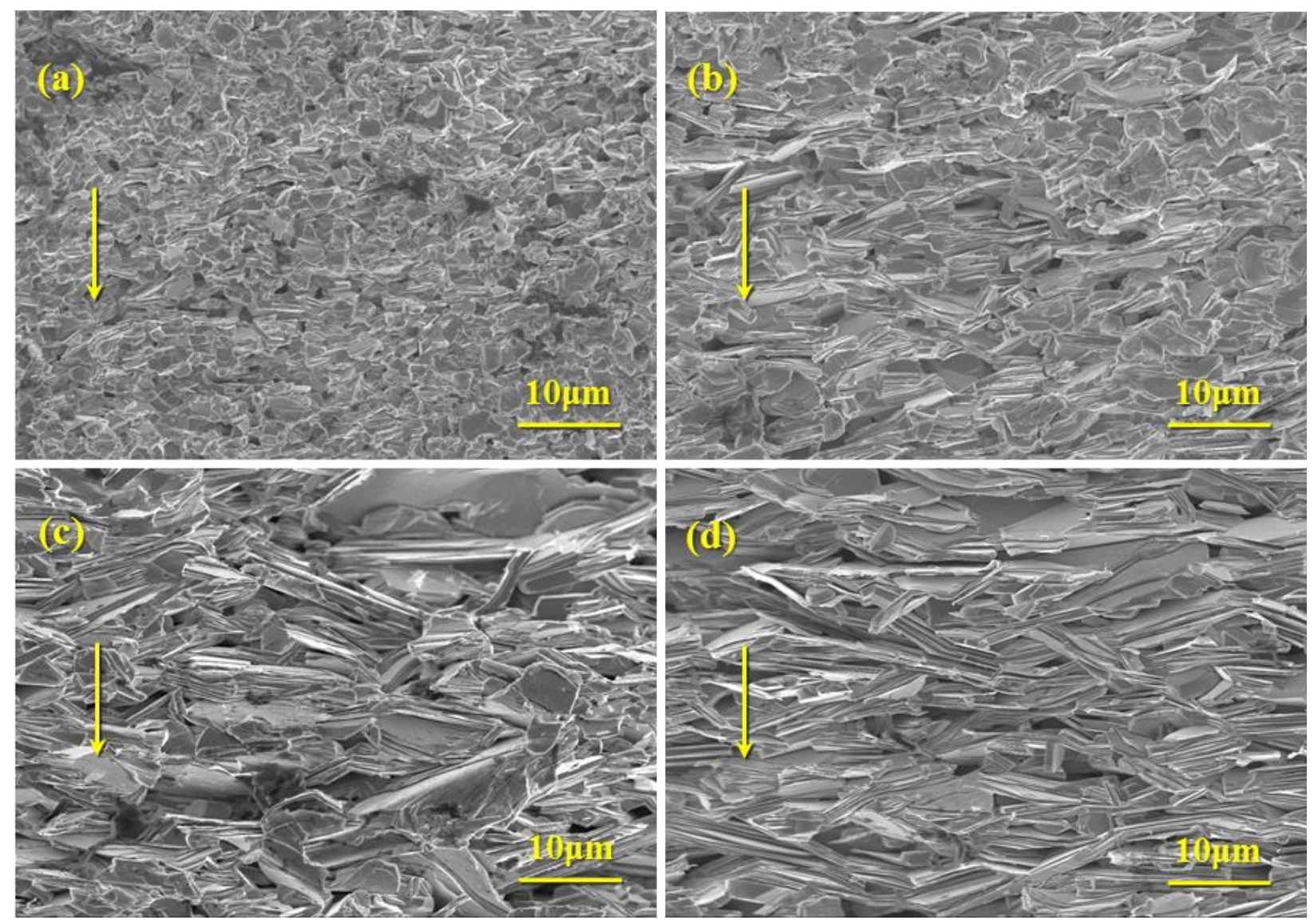

Figure 6-5: Cross-sectional SEM images from the fractured surface of $\mathrm{Ca}_{3} \mathrm{Co}_{4} \mathrm{O}_{9} \mathrm{Bi}_{x} \mathrm{~K}_{y}$ samples after sintering stage. (a) $\mathrm{Ca}_{3} \mathrm{Co}_{4} \mathrm{O}_{9}$, (b) $\mathrm{Ca}_{3} \mathrm{Co}_{4} \mathrm{O}_{9} \mathrm{~K}_{0.05}$, (c) $\mathrm{Ca}_{3} \mathrm{Co}_{4} \mathrm{O}_{9} \mathrm{~K}_{0.05} \mathrm{Bi}_{0.1}$, and (d) $\mathrm{Ca}_{3} \mathrm{Co}_{4} \mathrm{O}_{9} \mathrm{~K}_{0.05} \mathrm{Bi}_{0.15}$.

TEM examination was performed on the $\mathrm{Ca}_{3} \mathrm{Co}_{4} \mathrm{O}_{9} \mathrm{Bi}_{0.15} \mathrm{~K}_{0.05}$ sample having the best crystal texture. The TEM images of Figure 6-6 illustrate the nanolamella structure from two adjacent grains with different $c$-axes, where the arrows are in parallel direction to the $c$-axes. Most of the adjacent crystals have a large amount of low angle GBs between them. No secondary phase was present in the TEM results, meaning those secondary phases exhibited by XRD results could exist in only very small local areas. Additionally, there is not presence of secondary phases at the GBs. EDS was used to further determine the atomic ratio of the different elements and the chemistry on the GBs and crystal lattice of the dual-doped CCO. The spots in Figure 6-6 correspond to the location of EDS between the adjacent grains. 


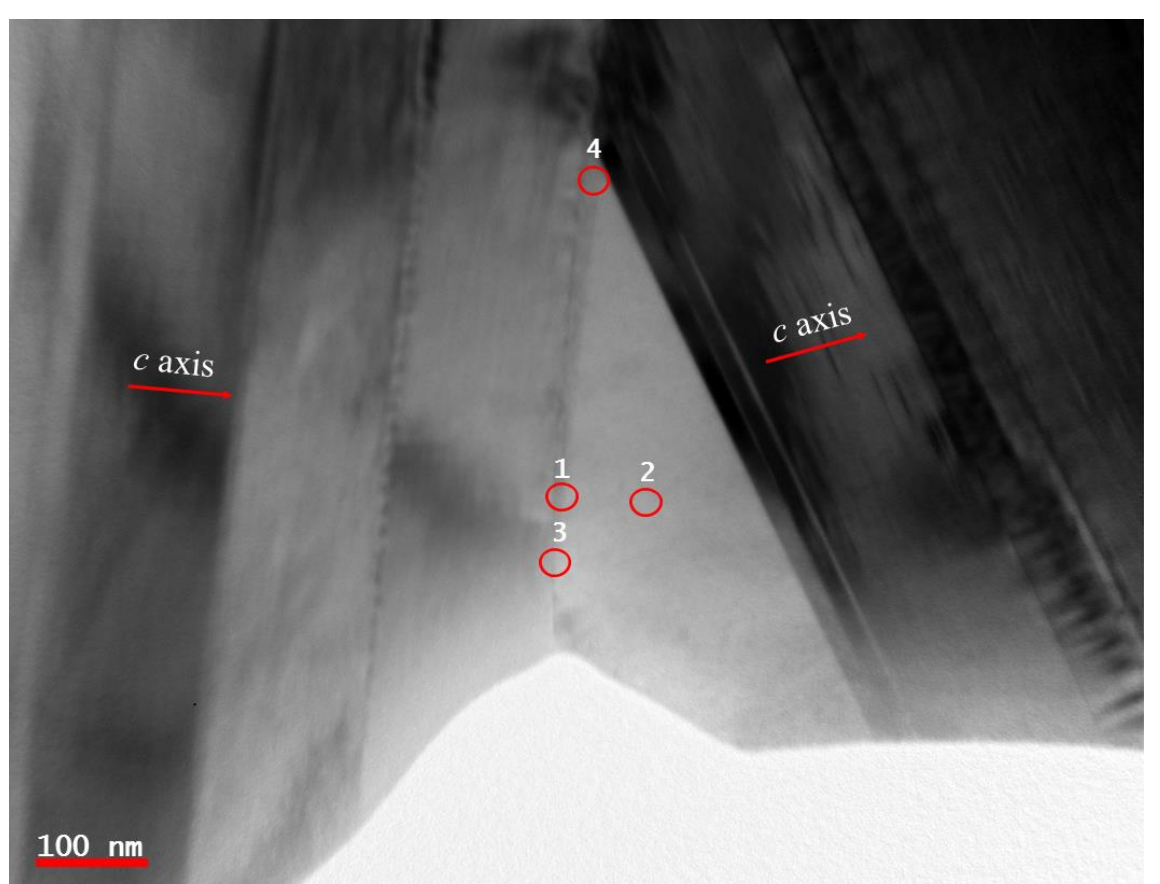

Figure 6-6: TEM images showing the nanostructure of the $\mathrm{Ca}_{3} \mathrm{Co}_{4} \mathrm{O}_{9} \mathrm{~K}_{0.05} \mathrm{Bi}_{0.15}$ sample. The grain boundary from the crystals with different c-axis orientations. The red circles on the TEM images indicate the electron beam sample areas for the EDS data acquisition.

The atomic ratio for each EDS spot is summarized in Table 6-3. Results confirmed the presence of $\mathrm{Bi}$ inside the lattice crystal and the depletion at the GBs. However, $\mathrm{K}$ was not found in many iterations for EDS analysis. Local areas were $\mathrm{K}$ is segregated at GBs must exist for the addition into the $\mathrm{CCO}$ as reported previously ${ }^{[26]}$, but the EDS results obtained are inconclusive due to the small concentration of $\mathrm{K}$ in the sample used for the dual non-stoichiometric addition.

Table 6-3: Chemistry of the grain interior and grain boundaries of $\mathrm{Ca}_{3} \mathrm{Co}_{4} \mathrm{O}_{9} \mathrm{Bi}_{0.15} \mathrm{~K}_{0.05}$ for different sites labeled in the TEM image.

\begin{tabular}{lllll}
\hline Atomic\% & $\mathrm{O}$ & $\mathrm{Ca}$ & $\mathrm{Co}$ & $\mathrm{Bi}$ \\
\hline 1 & 63.51 & 16.46 & 18.92 & 1.11 \\
2 & 61.11 & 17.91 & 19.70 & 1.28 \\
3 & 68.69 & 12.68 & 17.47 & 1.15 \\
4 & 61.68 & 16.29 & 20.78 & 1.25 \\
\hline
\end{tabular}




\section{5 Discussion}

There has not been previous research in Bi non-stoichiometric addition into CCO. However, Bi substitution ${ }^{[23]}$ of the Ca sites demonstrated a segregation of $\mathrm{Bi}$ at GBs. Whereas, combining the $\mathrm{Bi}$ substitution of the $\mathrm{Ca}$ sites with $\mathrm{Ba}$ non-stoichiometric addition produced segregation of $\mathrm{Ba}$ and depletion of $\mathrm{Bi}$ at the $\mathrm{GBs}^{[24]}$. In both cases ${ }^{[23,24]}, \mathrm{Bi}$ is the only present element in the crystal lattice. Notably, K and Ba have been reported to exist only segregated at the GBs. ${ }^{[25,26]}$ A selective segregation to the GBs has been reported due to the ion-size mismatch between the doping elements, where the bigger atoms occupies the GB and the smaller atom is displaced out of the GBs. ${ }^{[24]}$ In this paper, we further investigate the GBs effect of $\mathrm{Bi}$ and $\mathrm{K}$ using a dual nonstoichiometric approach to dope them into the $\mathrm{CCO}$.

$\mathrm{CCO}$ is compromise of two misfit layered subsystems aligned in the $c$-axis, the $\mathrm{CoO}_{2}$ layer being electrically conductive and the $\mathrm{CaCo}_{2} \mathrm{O}_{3}$ being electrically insulating. As a result, the resistivity in the $a-b$ basal plane of the monoclinic unit cell is significantly lower than along the $c$ axis. ${ }^{[9]}$ To improve the electrical properties it is therefore necessary to develop the crystal texture along the $a-b$ plane and enhance the better orientation perpendicular to the pressed direction. SEM images for the dual doped $\mathrm{Ca}_{3} \mathrm{Co}_{4} \mathrm{O}_{9} \mathrm{Bi}_{x} \mathrm{~K}_{y}$ shows the desired crystal texture and orientation. These microstructure improvements are directly linked to an enriched carrier mobility ${ }^{[31,32]}$. There has been reports on a substantial enhancement in carrier mobility originating from grain boundaries in oxides TE materials ${ }^{[18]}$, possibly serving as inter-granular conducting paths ${ }^{[14]}$. Additionally, low angle grain boundaries as those from TEM results do not fade the carrier mobility ${ }^{[15]}$. Moreover, $\sigma$ could be expressed as $\sigma=n e \mu$, where $\mu$ is the carrier mobility, $e$ is the elementary positive charge constant, and $n$ is the number density of electrons. For this reason, the reduction of $\rho$ in the $\mathrm{Ca}_{3} \mathrm{Co}_{4} \mathrm{O}_{9} \mathrm{Bi}_{x} \mathrm{~K}_{y}$ samples is result from an improved carrier mobility. At higher doping levels, the 
diminished electrical properties could be associated by the loss of carrier mobility due to formation of larger grains with random orientation.

The GBs engineering contribution to the $S$ in polycrystalline ceramics is reported to be directly associated to the conditions of the energy barrier established between adjacent grains. ${ }^{[15,16]}$ However, for the systems with dual dopants, there are complex interactions between different dopants that are added to the ceramics simultaneously. In the case of the dual non-stoichiometric addition in $\mathrm{Ca}_{3} \mathrm{Co}_{4} \mathrm{O}_{9} \mathrm{Bi}_{x} \mathrm{~K}_{y}$, the ratio between $\mathrm{Bi}$ and $\mathrm{K}$ have demonstrated a big role to enhance the $S$. In a ratio of $\mathrm{Bi} / \mathrm{K}<1.25 / 0.5$ the $S$ is greatly augmented, but in a ratio above $\mathrm{Bi} / \mathrm{K}>1.5 / 0.5$ the $S$ value remains close to that of the pristine CCO. Furthermore, the increase of $S$ could be associated to the selective filtering of low energy carriers at the GBs lead to a reduced carrier concentration which in turn improve $S^{[15,16]}$. The GBs segregation acts as a barrier layer mechanism in bulk semiconductor originated by a band offset among the grain boundary and the grain interior. ${ }^{[18]}$ The improvement in the $\mathrm{S}$ values from the ratio of $\mathrm{Bi} / \mathrm{K}<1.25 / 0.5$ in the dual nonstoichiometric samples infers a decrease in the carrier concentration of the $\mathrm{Ca}_{3} \mathrm{Co}_{4} \mathrm{O}_{9}$ ceramics. At high temperature $(T \rightarrow \infty)$ the Seebeck coefficient of the $\mathrm{Ca}_{3} \mathrm{Co}_{4} \mathrm{O}_{9}$ can be expressed with Heike's formula as ${ }^{[33]}$ :

$$
S_{(T \rightarrow \infty)}=-\frac{k_{B}}{e} \ln \left(\frac{g_{3}}{g_{4}} \frac{x}{1-x}\right)
$$

where $\mathrm{g}_{3}$ and $\mathrm{g}_{4}$ is the number of configuration of the $\mathrm{Co}^{3+}$ and $\mathrm{Co}^{4+}$ ions, respectively, and $\mathrm{x}$ is the fraction of $\mathrm{Co}^{4+}$ in the $\mathrm{CoO}_{2}$ layer. $k_{B}$ and $e$ are the Boltzmann constant and the electronic charge, respectively. Thus, the magnitude of $S$ increase with a reduction of $\mathrm{Co}^{4+}$ concentration. However, it is therefore implied that a higher ratio $\mathrm{Bi} / \mathrm{K}$ do reduce $\mathrm{Co}^{4+}$ concentration, and consequently the $S$ is not improved. 
The predominant component of polycrystalline $\mathrm{CCO}$ of the overall $\kappa$ is the contribution of $\kappa_{i}$ as showed in Figure 6-1(b). Enhancements in the electronic transport properties (leading to higher $\left.\kappa_{e}\right)$ take a smaller impact on the overall $\kappa$. Point-defect phonon scattering is the main driving force in our material due to the mass difference between the host atoms and the dopants in the material rather than the influence of a phase layer ${ }^{[17]}$, dislocations strains ${ }^{[4]}$, or doped-rich GBs regions ${ }^{[18]}$. Such point defects should scatter short-wavelength phonons effectively ${ }^{[20]}$ due to a shortened phonon mean free path. However, owing to the light atomic mass of $\mathrm{K}$, its addition into the $\mathrm{CCO}$ has a negative impact on both $\kappa_{i}$ and $\kappa_{e}$. More notably, since Bi holds ionic radius similar to the host ions while being much heavier in atomic mass, its addition in the dual non-stoichiometric approach offset the increased $\kappa$ with the increasing doping level of Bi. Such results are consistent with previous research on $\mathrm{Bi}$ doped $\mathrm{CCO}^{[23,24]}$. The main improvement comes from the reduced lattice contribution due to the presence of $\mathrm{Bi}$ at the $\mathrm{CCO}$ lattice.

Overall, the $Z T$ of the best concentration of dual non-stoichiometric addition $\mathrm{Ca}_{3} \mathrm{Co}_{4} \mathrm{O}_{9} \mathrm{Bi}_{x} \mathrm{~K}_{y}$ was enhanced to a value of 0.44 at $1073 \mathrm{~K}$, which represents a 30\% increase with the undoped CCO. The increased $Z T$ value is achieved by the enriched electronic properties of the material, such improvement lead to an electrical PF value of $0.77 \mathrm{mWm}^{-1} \mathrm{~K}^{-2}$ at $1073 \mathrm{~K}$. Notwithstanding, the overall $\kappa$ is not improved in comparison to the pristine CCO. However Bi remains a viable candidate to promote phonon scattering events in the CCO.

\section{6 Conclusion}

For the dual non-stoichiometric addition system, there are complex interactions between the different dopants that are added to the CCO ceramics simultaneously. Their interactions affected both the electrical power factor to a peak value of $0.77 \mathrm{mWm}^{-1} \mathrm{~K}^{-2}$ at $1073 \mathrm{~K}$ and negatively affecting the thermal conductivity to a value of $1.85 \mathrm{~W} / \mathrm{mK}$, such influence is related to the nature 
and level of the dopants. Doping both $\mathrm{Bi}$ and $\mathrm{K}^{+}$via non-stoichiometric addition into the $\mathrm{CCO}$ boosted the electrical PF. Moreover, the positive influence of a moderate ratio of $\mathrm{Bi} / \mathrm{K}^{+}$to the Seebeck values was observed with $\sim 10 \%$ improvement at $320 \mathrm{~K}$. When the ratio is below $0.125 / 0.05$ for $\mathrm{Bi} / \mathrm{K}^{+}, S$ is enhanced a value $150 \mu \mathrm{VK}^{-1}$. The overall $\mathrm{ZT}$ was improved by the best concentration to a value of 0.44 at $1073 \mathrm{~K}$ which represents a $30 \%$ increase to the value of the pristine CCO. However, the biggest challenge for the designed material was the high $\kappa$ values due to the inclusion of the light atomic mass of $\mathrm{K}^{+}$. which produced a detrimental $10 \%$ increase in the thermal transport properties. Evidence of $\mathrm{Bi}$ depletion at the $\mathrm{GBs}$ in the $\mathrm{Ca}_{3} \mathrm{Co}_{4} \mathrm{O}_{9} \mathrm{Bi}_{x} \mathrm{~K}_{y}$ best concentration was present in the EDS analysis. Additionally, TEM revealed the existence of Bi at the crystal lattice, while XRD results also showed an increase in the lattice parameter of the unit cell, thus, likely substitution of $\mathrm{Bi}$ at $\mathrm{Ca}^{2+}$ sites exist. The GBs modification by the dual addition and the incorporation of $\mathrm{Bi}$ in the crystal lattice improved notably texture and alignment in the CCO crystals, such improvement yielded a lower $\rho$ which is a $39 \%$ reduction compared to the pristine material. GBs segregation (and depletion) of dopants through GBs engineering could play an important role in improving both the electronic and thermal properties in $\mathrm{CCO}$ ceramics exploration to remain competitive versus conventional TE materials, such properties produced a $40 \%$ improvement of the electrical power factor. Research on the use of multiple dopants is further carried to better understand the complexity in the influence of polycrystalline $\mathrm{Ca}_{3} \mathrm{Co}_{4} \mathrm{O}_{9} \mathrm{GBs}$. 


\section{References}

1. Chu, S. and A. Majumdar, Opportunities and challenges for a sustainable energy future. Nature, 2012. 488: p. 294.

2. Chu, S., Y. Cui, and N. Liu, The path towards sustainable energy. Nature Materials, 2016. 16: p. 16.

3. Koumoto, K., et al., Thermoelectric Ceramics for Energy Harvesting. Journal of the American Ceramic Society, 2013. 96(1): p. 1-23.

4. Kim, S.I., et al., Dense dislocation arrays embedded in grain boundaries for highperformance bulk thermoelectrics. Science, 2015. 348(6230): p. 109.

5. Joshi, G., et al., Enhanced Thermoelectric Figure-of-Merit in Nanostructured p-type Silicon Germanium Bulk Alloys. Nano Letters, 2008. 8(12): p. 4670-4674.

6. Chen, Z.-G., et al., Nanostructured thermoelectric materials: Current research and future challenge. Progress in Natural Science: Materials International, 2012. 22(6): p. 535-549.

7. Fergus, J.W., Oxide materials for high temperature thermoelectric energy conversion. Journal of the European Ceramic Society, 2012. 32(3): p. 525-540.

8. Woermann, E. and A. Muan, Phase equilibria in the system CaO-cobalt oxide in air. Journal of Inorganic and Nuclear Chemistry, 1970. 32(5): p. 1455-1459.

9. Shikano, M. and R. Funahashi, Electrical and thermal properties of single-crystalline $\left(\mathrm{Ca}_{2} \mathrm{CoO}_{3}\right)_{0.7} \mathrm{CoO}_{2}$ with a $\mathrm{Ca}_{3} \mathrm{Co}_{4} \mathrm{O}_{9}$ structure. Applied Physics Letters, 2003. 82(12): p. 1851-1853.

10. Snyder, G.J. and E.S. Toberer, Complex thermoelectric materials. Nature Materials, 2008. 7: p. 105.

11. Bell, L.E., Cooling, Heating, Generating Power, and Recovering Waste Heat with Thermoelectric Systems. Science, 2008. 321(5895): p. 1457.

12. Dillon, S.J., M.P. Harmer, and J. Luo, Grain boundary complexions in ceramics and metals: An overview. JOM, 2009. 61(12): p. 38-44.

13. Li, G., et al., Grain Boundaries Softening Thermoelectric Oxide BiCuSeO. ACS Applied Materials \& Interfaces, 2018. 10(7): p. 6772-6777.

14. Muthiah, S., et al., Conducting grain boundaries enhancing thermoelectric performance in doped Mg 2 Si. Applied Physics Letters, 2013. 103(5): p. 053901.

15. Meng, X., et al., Grain Boundary Engineering for Achieving High Thermoelectric Performance in n-Type Skutterudites. Advanced Energy Materials, 2017. 7(13): p. 1602582.

16. Hossein-Babaei, F., S. Masoumi, and A. Noori, Linking thermoelectric generation in polycrystalline semiconductors to grain boundary effects sets a platform for novel Seebeck effect-based sensors. Journal of Materials Chemistry A, 2018. 6(22): p. 10370-10378.

17. Zong, P.-a., et al., Skutterudite with graphene-modified grain-boundary complexion enhances zT enabling high-efficiency thermoelectric device. Energy \& Environmental Science, 2017. 10(1): p. 183-191.

18. Mehdizadeh Dehkordi, A., et al., Large Thermoelectric Power Factor in Pr-Doped $\mathrm{SrTiO}_{3-\delta}$ Ceramics via Grain-Boundary-Induced Mobility Enhancement. Chemistry of Materials, 2014. 26(7): p. 2478-2485.

19. Zhang, Q., et al., Realizing high-performance thermoelectric power generation through grain boundary engineering of skutterudite-based nanocomposites. Nano Energy, 2017. 41: p. 501-510. 
20. Mun, H., et al., Boundary Engineering for the Thermoelectric Performance of Bulk Alloys Based on Bismuth Telluride. ChemSusChem, 2015. 8(14): p. 2312-2326.

21. Poudel, B., et al., High-Thermoelectric Performance of Nanostructured Bismuth Antimony Telluride Bulk Alloys. Science, 2008. 320(5876): p. 634.

22. Mao, J., et al., Manipulation of ionized impurity scattering for achieving high thermoelectric performance in n-type $\mathrm{Mg}_{3} \mathrm{Sb}_{2}$-based materials. Proceedings of the National Academy of Sciences of the United States of America, 2017. 114(40): p. 10548-10553.

23. Boyle, C., et al., Grain boundary segregation and thermoelectric performance enhancement of bismuth doped calcium cobaltite. Journal of the European Ceramic Society, 2016. 36(3): p. 601-607.

24. Boyle, C., et al., Competing dopants grain boundary segregation and resultant seebeck coefficient and power factor enhancement of thermoelectric calcium cobaltite ceramics. Ceramics International, 2017. 43(14): p. 11523-11528.

25. Carvillo, P., et al., Thermoelectric Performance Enhancement of Calcium Cobaltite through Barium Grain Boundary Segregation. Inorganic Chemistry, 2015. 54(18): p. 90279032.

26. Romo-De-La-Cruz, C., et al., Role of oversized dopant potassium on the nanostructure and thermoelectric performance of calcium cobaltite ceramics. Sustainable Energy \& Fuels, 2018. 2(4): p. 876-881.

27. Sotelo, A., et al., Improvement of thermoelectric properties of $\mathrm{Ca}_{3} \mathrm{Co}_{4} \mathrm{O}_{9}$ using soft chemistry synthetic methods. Journal of the European Ceramic Society, 2012. 32(10): p. 2415-2422.

28. Chen, S., et al., Effect of precursor calcination temperature on the microstructure and thermoelectric properties of $\mathrm{Ca}_{3} \mathrm{Co}_{4} \mathrm{O}_{9}$ ceramics. Journal of Sol-Gel Science and Technology, 2012. 64(3): p. 627-636.

29. Boyle, C., et al., Improving the thermoelectric performance and thermal stability of $\mathrm{Ca}_{3} \mathrm{Co}_{4} \mathrm{O}_{9}$ ceramics by sintering in oxygen atmosphere. Journal of Sol-Gel Science and Technology, 2018. 85(3): p. 712-722.

30. Delorme, F., et al., Thermoelectric properties of $\mathrm{Ca}_{3} \mathrm{CO}_{4} \mathrm{O}_{9}-\mathrm{Co}_{3} \mathrm{O}_{4}$ composites. Ceramics International, 2015. 41(8): p. 10038-10043.

31. Mikami, M., et al., Enhancement of Electrical Properties of the Thermoelectric Compound $\mathrm{Ca}_{3} \mathrm{Co}_{4} \mathrm{O}_{9}$ through Use of Large-grained Powder. Journal of Materials Research, 2011. 20(9): p. 2491-2497.

32. Guilmeau, E., et al., Thermoelectric properties-texture relationship in highly oriented $\mathrm{Ca}_{3} \mathrm{Co}_{4} \mathrm{O}_{9}$ composites. Applied Physics Letters, 2004. 85(9): p. 1490-1492.

33. Cutler, M. and N.F. Mott, Observation of Anderson Localization in an Electron Gas. Physical Review, 1969. 181(3): p. 1336-1340. 


\section{Chapter 7. Influence of Terbium Depletion at the Grain Boundaries on the Nanostructure and Thermoelectric Performance of $\mathrm{Ca}_{3} \mathrm{CO}_{4} \mathrm{O}_{9}$}

\section{1 Reducing thermal transport properties with Terbium}

The influence of the substitution of Terbium for Calcium atoms causes the reduction of thermal conductivity and a substantial enhancement of Seebeck simultaneously, whereas this substitution also results in the increase on the electrical resistivity. Terbium $(\mathrm{Tb})$ doped $\mathrm{Ca}_{3} \mathrm{Co}_{4} \mathrm{O}_{9}$ ceramic specimens were prepared by conventional cold-pressing technique and their thermoelectric properties were analyzed from room temperature to $1073 \mathrm{~K}$. The heavier atomic mass of $\mathrm{Tb}$ is beneficial for the suppression of the contribution of thermal lattice. Furthermore, the XPS analysis shows that the substitution of $\mathrm{Tb}$ ions in $\mathrm{Ca}_{3} \mathrm{Co}_{4} \mathrm{O}_{9}$ causes a reduction on the concentration of $\mathrm{Co}^{4+}$ ions which lead to a decrease in the concentration, and thus, the meaningful increase of the Seebeck. On the other hand, $\mathrm{Tb}$ substitution reduces significantly the $\mathrm{Ca}_{3} \mathrm{Co}_{4} \mathrm{O}_{9}$ crystal size and promote more arbitrary crystal orientation. TEM shows $\mathrm{Tb}$ depletion at the grain boundaries of the adjacent $\mathrm{Ca}_{3} \mathrm{Co}_{4} \mathrm{O}_{9}$ crystal. Nonetheless, with a low concentration of $\mathrm{Tb}$ substitution the dimensionless figure of merit ZT can reach 0.38 at $1073 \mathrm{~K}$, which represents a $10 \%$ increase in comparison to the baseline value.

\section{2 Background \& motivation for $\mathrm{Ca}_{3-\mathrm{x}} \mathrm{Tb}_{\mathrm{x}} \mathrm{Co}_{4} \mathrm{O}_{9}$ material.}

Current research has revealed that $72 \%$ of the global energy consumption is lost after conversion. About $60 \%$ of the waste heat is comprised below $373 \mathrm{~K}$, nevertheless, temperature above $573 \mathrm{~K}$ has demonstrated a greater waste heat potential due to the Carnot's efficiency ${ }^{[1]}$. Thermoelectric (TE) materials provide an excellent opportunity to harvest the energy of the wasted heat from multiple temperature sources. ${ }^{[2]}$ Conventional $p$-type TE material already possess a figure of merit $(\mathrm{ZT})$ value in excess of $1^{[3,4]}$. Conventional p-type TE materials hold several 
disadvantages in some commercial application since most of them are not stable at high temperature, they are mostly composed of toxic materials, and are more expensive to fabricate ${ }^{[5]}$.

The energy conversion efficiency of the TE materials is described by the ZT, which is defined as $Z T=S^{2} /(\rho \kappa) T$, where $S, \rho, S^{2} / \rho$, and $\kappa$ are the Seebeck coefficient, electrical resistivity, electrical power factor(PF) and thermal conductivity, respectively. Where, the total $\kappa$ in a material is a contribution of the electronic thermal conductivity $\left(\kappa_{e}\right)$ and the lattice thermal conductivity $\left(\kappa_{i}\right)$.

In contrast to conventional p-type $\mathrm{TE}$ material, $\mathrm{Ca}_{3} \mathrm{Co}_{4} \mathrm{O}_{9}(\mathrm{CCO})$ is stable at high temperature in air ${ }^{[6]}$, while being composed of materials that are cheap to fabricate ${ }^{[7]}$. CCO represents a viable alternative to be used in TE devices for high temperature harvesting with a ZT value of 0.87 at 973 $\mathrm{K}$ for its single crystal form ${ }^{[8]}$. Nonetheless, the ZT performance of the polycrystalline $\mathrm{CCO}$ has never approached such value, and most of the reported literature sits below a ZT value of 0.3. ${ }^{[9]}$ The biggest reason for such low performance resides in its low electrical power factor (PF). Polycrystalline CCO suffers from a reduction in both the Seebeck and specially the $\rho$.

The enhancement of the electrical PF -specially from an improved Seebeck- is from great importance in the exploration and further improvement of the performance of polycrystalline CCO. Recently, Saini et a ${ }^{[10]}$ reported the big impact of the substitution of $\mathrm{Ca}$ ions by the heavier $\mathrm{Tb}$ ions. It was reported a large improvement of both the $\rho$ and the electrical PF, with a simultaneous reduction of $\kappa$. More notably, the research reports the highest ZT achieved for the CCO polycrystalline TE material with a value of 0.74 . Unlike the previous reported work on Tb doped $\mathrm{CCO}$, our investigation starts with previously reported optimal conditions for the calcination ${ }^{[11]}$ and the sintering ${ }^{[12]}$ stages. Moreover, contrasting the solid state route used in the mentioned report, our work uses sol-gel chemical route to significantly reduce the secondary phase content in $\mathrm{CCO}^{[13]}$, since common secondary phases such as $\mathrm{Co}_{3} \mathrm{O}_{4}$ decrease the performance of the $\mathrm{CCO}^{[14]}$. 
In this work, the chemistry analysis was further explored under TEM and it revealed the depletion of $\mathrm{Tb}$ at the Grain Boundaries (GBs) of $\mathrm{Ca}_{3} \mathrm{Co}_{4} \mathrm{O}_{9}$ grains with different c-axis. Prior investigation showed a selective segregation and depletion at the GBs, where the main driving force linked to the strain effect, in which smaller-sized atoms are depleted at the GBs and bigger over-sized atoms segregate to the GBs ${ }^{[15]}$. Tb is depleted at the GBs, which is consistent because it is substituting the bigger $\mathrm{Ca}$ ion.

$\mathrm{Tb}$ substitution into the $\mathrm{CCO}$ enhance thermoelectric performance of the dimensionless figure of merit ZT to a value of 0.38 at $1073 \mathrm{~K}$, which represents a $10 \%$ increase in comparison to the baseline value. Although, $\mathrm{Tb}$ does not improve the crystal texture nor the crystal orientation, it greatly improves the Seebeck value, and the obtained performance might be the start of suitable dopant to further explore dual doping possibilities as it is explained in the next chapters.

\section{3 Experimental procedure}

The polycrystalline ceramic samples were prepared by the conventional chemical sol-gel route with a chemical composition $\mathrm{Ca}_{3-\mathrm{x}} \mathrm{Tb}_{\mathrm{x}} \mathrm{Co}_{4} \mathrm{O}_{9}(x=0,0.05,0.30$, and 0.50$)$. The precursor powders were obtained mixing chemical reagents: $\mathrm{Ca}\left(\mathrm{NO}_{3}\right)_{2} \cdot 4 \mathrm{H}_{2} \mathrm{O}$ (99\%, Acros Organics), $\mathrm{Co}\left(\mathrm{NO}_{3}\right)_{2} \cdot 6 \mathrm{H}_{2} \mathrm{O}\left(99 \%\right.$, Acros Organics), and $\mathrm{Tb}\left(\mathrm{NO}_{3}\right)_{3} \cdot 6 \mathrm{H}_{2} \mathrm{O}$ (99.9\% Strem Chemicals) in deionized water according to the stoichiometric chemical compositions. Citric acid (BDH Chemical), ethylene glycol, and polyethylene glycol were dissolved in the solution to polymerize the mixture. Nitric acid was included to the solution to promote the sol-gel synthesis, nitrate salts decomposition, and the new compound formation. The sol-gel mixture was mechanically stirred at $353 \mathrm{~K}$ for $3 \mathrm{~h}$. The obtained sol-gel mixture was ashed at $773 \mathrm{~K}$ for $2 \mathrm{hr}$ in air inside a box furnace. The subsequent ashes were ball-milled in ethyl alcohol in a stage with zirconia balls and then dried at room temperature with a final manually ground stage after that to obtain uniform 
grain size in the powder. The reasonable homogeneous ashes were calcined at $973 \mathrm{~K}$ for $4 \mathrm{~h}$ in a tube furnace with a constant oxygen flow. The calcined powders were uniaxially pressed into round pellets under a $1 \mathrm{Gpa}$ pressure at room temperature. The bulk samples were sintered at 1233 $\mathrm{K}$ in a tube furnace with a constant oxygen flow and then cut into rectangular pellets to obtain the final desired sample for either the electrical or thermal measurements. The apparent densities for all bulk samples are listed in Table 7-1.

Table 7-1: Apparent density of the $\mathrm{Ca}_{3-x} \mathrm{~Tb}_{x} \mathrm{Co}_{4} \mathrm{O}_{9}$ samples.

\begin{tabular}{ll}
\hline $\mathrm{Ca}_{3-\mathrm{x}} \mathrm{Tb}_{\mathrm{x}} \mathrm{Co}_{4} \mathrm{O}_{9} \mathrm{Bi}_{\mathrm{y}}$ & Average Apparent density $(\mathrm{g} / \mathrm{cm} 3)$ \\
\hline $\mathrm{x}=0 \quad \mathrm{y}=0$ & 3.883 \\
$\mathrm{x}=0.05 \mathrm{y}=0$ & 4.182 \\
$\mathrm{x}=0.30 \mathrm{y}=0$ & 4.477 \\
$\mathrm{x}=0.50 \mathrm{y}=0$ & 4.571 \\
\hline
\end{tabular}

The electrical resistivity $(\rho)$ and absolute Seebeck coefficient $(S)$ were measured at the same time by the four-terminal method using a Linseis LSR-1100 unit. The measurements were performed perpendicular to the pressed direction from $323 \mathrm{k}$ to $1073 \mathrm{~K}$ under a low pressure $\mathrm{He}$ environment. The thermal conductivity was calculated using the equation $\kappa=\lambda C_{p} \rho m$, where $C_{p}, \lambda$, and $m$ are the specific heat, thermal diffusivity, and mass density, respectively. The $C p$ and $\lambda$ values were obtained in the range 323 to $1073 \mathrm{~K}$ using a Linseis Laser Flash Analyzer 1200. The measurements were also performed perpendicular to the pressed direction and samples were analyzed under a low-pressure air environment. X-Ray powder Diffraction (XRD) analysis was performed using a PANatycal X'Pert Pro XRD unit for crystal phase and lattice parameter determination purposes. The ground powders from the sintered samples were used in the XRD analysis using $\mathrm{Cu} \mathrm{K}$-alpha radiation, $45 \mathrm{kV}$ voltage and $40 \mathrm{~mA}$ current at room temperature. The cross-section and plan-view morphology of the sintered pellets was observed using a HitachiS4700F Scanning Electron Microscope (SEM) unit. Transmission electron microscopy (TEM) 
samples were prepared from sintered pellets by mechanically polishing and ion milling in a liquidnitrogen-cooled holder. TEM imaging including Energy dispersive spectroscopy (EDS) and high resolution TEM were performed in a JEM-2100 operated at $200 \mathrm{kV}$. X-ray Photoelectron Spectroscopy (XPS) was performed using Physical Electronics PHI 5000 VersaProbe system. Multipak XPS software was used to analyze the obtained data.

\section{4 Results}

The temperature dependence of the electrical properties are shown in Figure 7-1 (a) $\rho-T$, (b) $S$ $T$, and (c) $S^{2} / \rho-T$ for different $\mathrm{Ca}_{3-\mathrm{x}} \mathrm{Tb}_{\mathrm{x}} \mathrm{C}_{4} \mathrm{O}_{9}$ concentrations. The inset in Figure 7-1 (a) shows the direction in which the electrical measurements were performed for $\rho-T, S-T$, and $S^{2} / \rho-T$. The $\rho$-T curves in Figure 7-1 (a) for $\mathrm{x}=0.3$ and 0.5 show a semiconductor-like behavior and the $\rho$ decreases as the temperature increases. With the increase in Tb doping level the magnitude of $\rho$ increases. At $x=0.05$, the $\rho$-T properties have a metallic-like behavior and a transition to a semiconductor-like behavior around $473 \mathrm{~K}$, reaching a $\rho$ slightly lower in magnitude at high temperature compared to the pristine material.

There is a simultaneous impact on the $\mathrm{S}$ by the doping of $\mathrm{Tb}$ into $\mathrm{CCO}$. As the Tb doping level increases the magnitude of the $S$ is increased in the broad temperature range, as shown in Figure 7-1 (b). Compared to the pristine CCO ceramics, doping $\mathrm{Tb}$ is a notably enhancer of $\mathrm{S}$ even with a small doping level. For the highest doping level $\mathrm{Ca}_{2.5} \mathrm{~Tb}_{0.5} \mathrm{Co}_{4} \mathrm{O}_{9}$, the $\mathrm{S}$ reaches a value of 191 $\mu \mathrm{V} / \mathrm{K}$ at $323 \mathrm{~K}$, which represents almost a $45 \%$ increase compared to the baseline value. However, as stated in the $\rho$-T curves, Tb doping suffers of elevated $\rho$ values. The electrical PF, shown in Figure 7-1 (c) for $\mathrm{Ca}_{3-\mathrm{x}} \mathrm{Tb}_{\mathbf{x}} \mathrm{Co}_{4} \mathrm{O}_{9}$ shows that a doping level of $x=0.05$ is slightly beneficial to the material since increasing the $\mathrm{Tb}$ doping level produces a lower PF than the initial baseline due to the reduced electrical conductivity. 


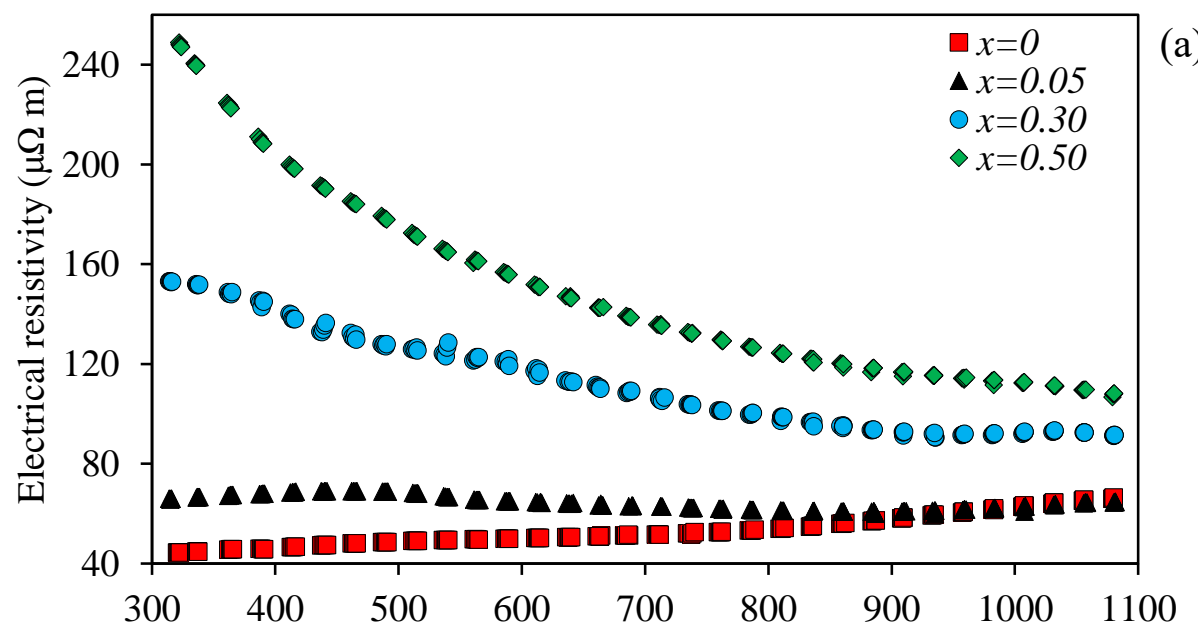

(a)
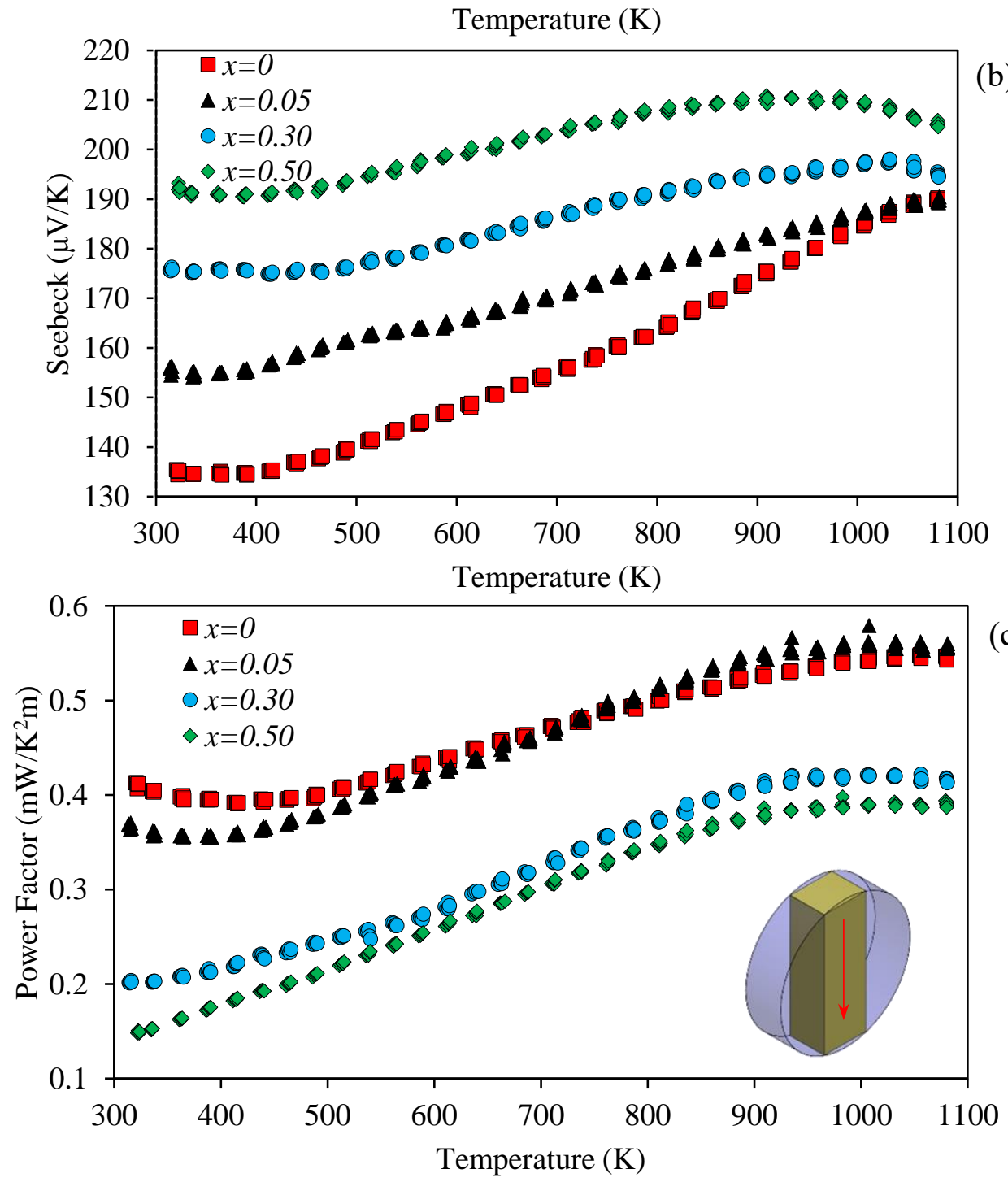

Figure 7-1 (a) $\rho-T$, (b) $S$-T, and (c) $S^{2} / \rho$-T show the temperature dependence of electrical properties for $\mathrm{Ca}_{3-x} \mathrm{~Tb}_{x} \mathrm{Co}_{4} \mathrm{O}_{9}$. Inset figure shows the direction of the performed measurements. 

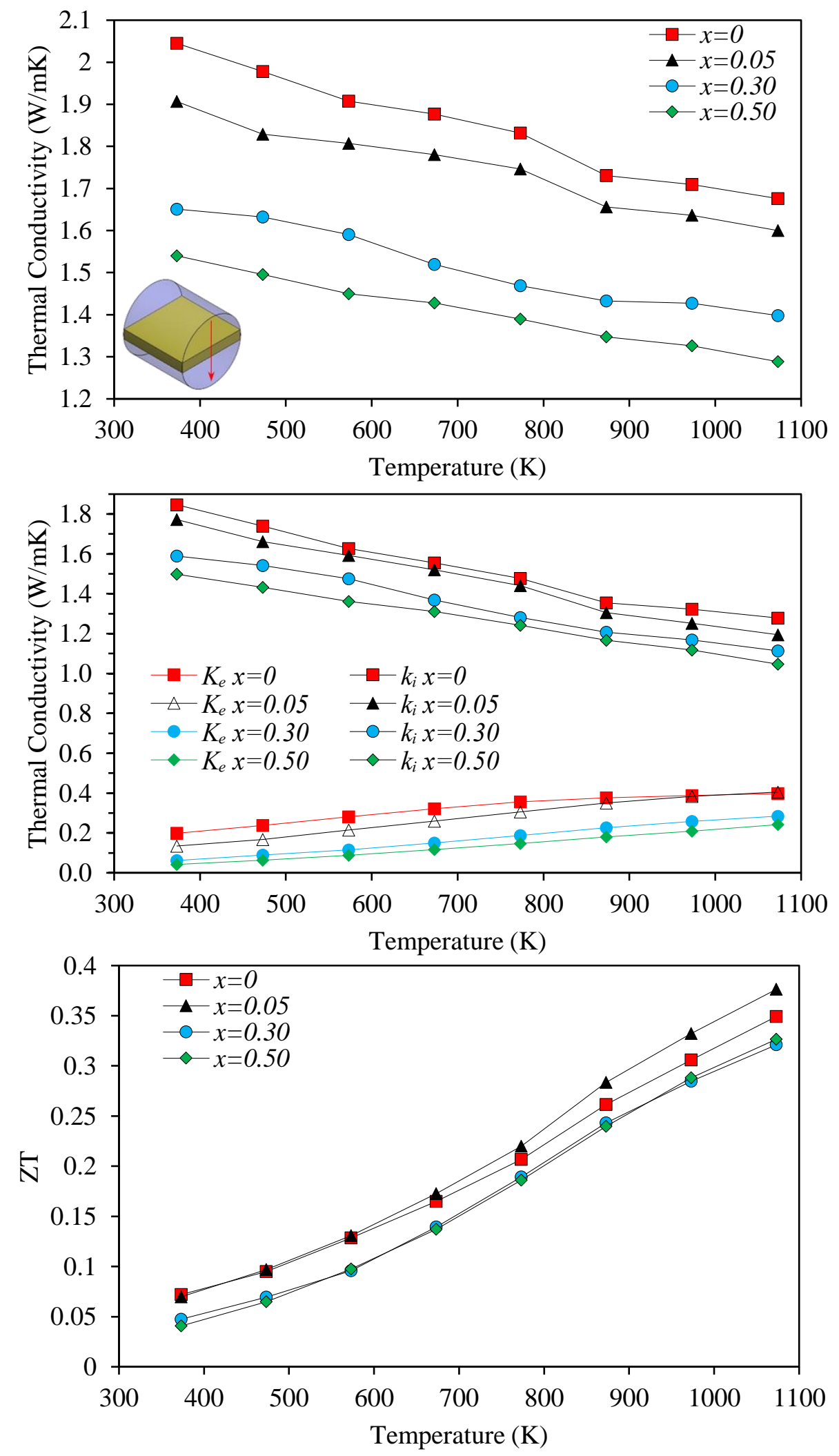

Figure 7-2: Temperature dependence of: (a) $\kappa-T$, (b) $\kappa_{e}$ and $\kappa_{i}$, and (c) ZT for $\mathrm{Ca}_{3-x} \mathrm{~Tb}_{x} \mathrm{Co}_{4} \mathrm{O}_{9}$.

Inset figure in (d) shows the direction of the performed measurements for $\kappa-T$. 
The thermal conductivity and ZT of the ceramics analyzed are shown in Figure 7-2 (a) $\kappa-T$, (b) $\kappa_{\mathrm{e}}-T$ and $\kappa_{\mathrm{i}}-T$, and (c) $Z T$ for different $\mathrm{Ca}_{3-\mathrm{x}} \mathrm{Tb}_{\mathrm{x}} \mathrm{Co}_{4} \mathrm{O}_{9}$ samples. The $\mathrm{Tb}$ concentration level plays a fundamental role in the thermal properties of the $\mathrm{Ca}_{3} \mathrm{Co}_{4} \mathrm{O}_{9}$ samples. With the substitution of $\mathrm{Ca}$ by heavier atoms of $\mathrm{Tb}$ the magnitude of $\kappa$ is continuously reduced with the increasing $\mathrm{Tb}$ doping level, as shown in Figure 7-2 (a). The cause of the distinctively low $\kappa$ is due to the gradual reduction of both $\kappa_{\mathrm{e}}$ and $\kappa_{\mathrm{i}}$ contribution by increasing Tb doping level.

The figure of merit is enhanced slightly with the stoichiometric substitution of $\mathrm{Tb}$, resulting in a $Z T$ of 0.38 at $1073 \mathrm{~K}$, achieved only by a small $\mathrm{Tb}$ concentration. Adding a higher concentration of $\mathrm{Tb}$ decreases the $Z T$ performance. The improvement of the $Z T$ by the $\mathrm{Tb}$ dopant is due to the improved $S$ and the reduction of $\kappa$.

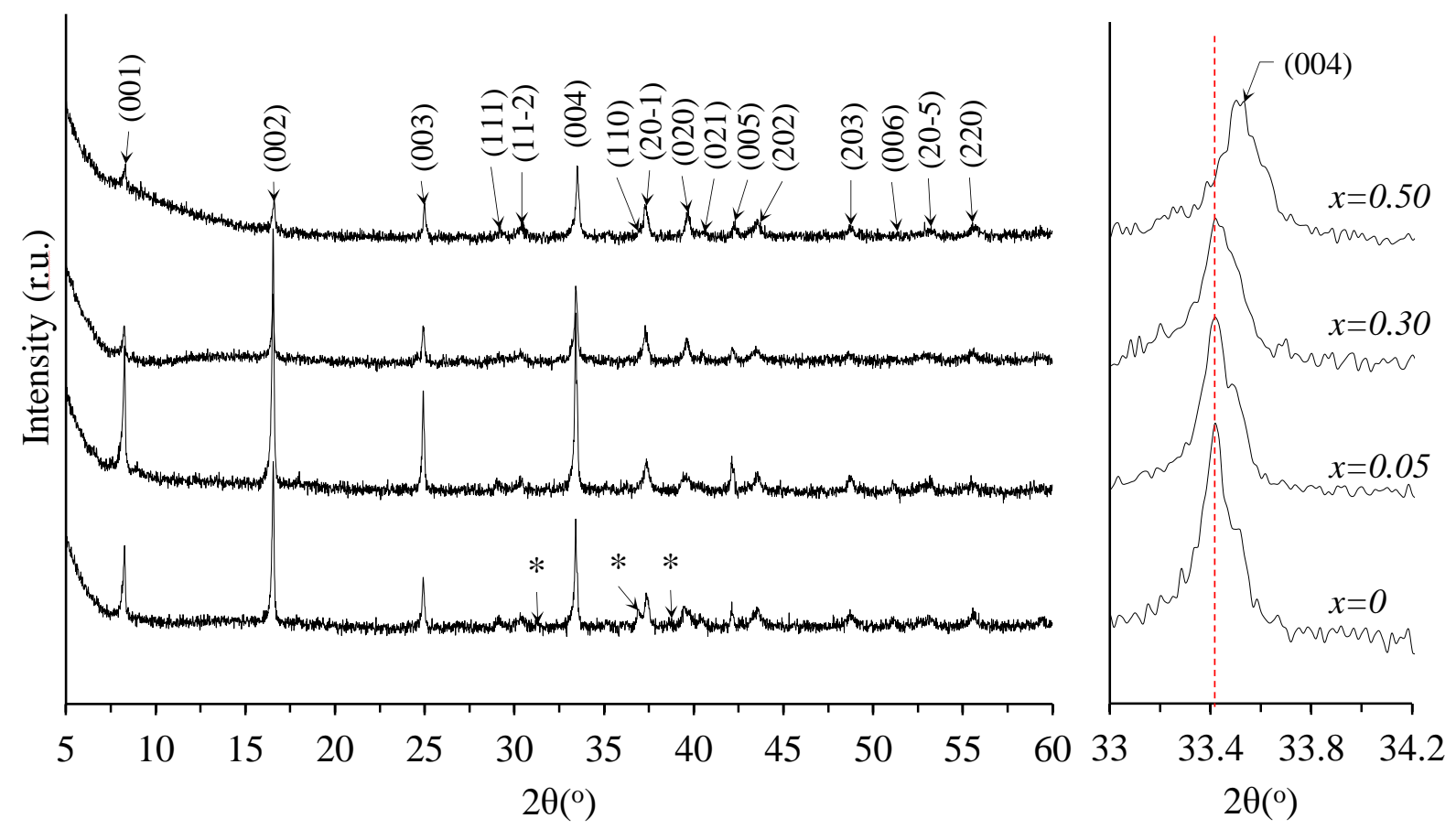

Figure 7-3: $X R D$ powder diffraction patterns for $\mathrm{Ca}_{3-x} \mathrm{~Tb}_{x} \mathrm{Co}_{4} \mathrm{O}_{9}(x=0,0.05,0.30$, and 0.50) samples. In all samples, monoclinic $\mathrm{Ca}_{3} \mathrm{Co}_{4} \mathrm{O}_{9}$ was observed. Nonetheless, a minor amount of $\mathrm{Co}_{3} \mathrm{O}_{4}$ was observe as indicated by the marked peaks. (004) diffraction peak showing the shift to a higher Bragg's angle. Red line correspond to position of peak in the undoped $\mathrm{Ca}_{3} \mathrm{Co}_{4} \mathrm{O}_{9}$. 
XRD patterns of $\mathrm{Ca}_{3-x} \mathrm{~Tb}_{x} \mathrm{Co}_{4} \mathrm{O}_{9}$ samples obtained at room temperature conditions from ground powders of sintered pellets are shown in Figure 7-3. The XRD patterns have been plotted with a minor offset with the purpose of a better visualization. The corresponding calculated lattice parameter values for samples with different doping level of the single doping $\mathrm{Ca}_{3-x} \mathrm{~Tb}_{x} \mathrm{Co}_{4} \mathrm{O}_{9}$ are listed in Table 7-2. Figure 7-3 (a) shows that the stoichiometric substitution Tb produces single phase $\mathrm{CCO}$ ceramics. However, there is a small presence of $\mathrm{C}_{3} \mathrm{O}_{4}$ secondary phase in the pristine CCO. Additionally, Figure 7-3 (b) confirms Tb substitution produces a slight shift to a higher Bragg's angle of the peaks for the different concentrations which indicates that $\mathrm{Tb}$ has been incorporated to the crystal lattice. Consequently, with the increase of the Tb doping level, there is a decrease in the lattice parameter values of $a, b_{1}, c$, and $\beta$, which is accompanied by the increase in the lattice parameter $b_{2}$, as listed in Table 7-2.

Table 7-2: Lattice parameters $a, b_{1}, b_{2}, c$, and $\beta$ for $\mathrm{Ca}_{3-x} \mathrm{~Tb}_{x} \mathrm{Co}_{4} \mathrm{O}_{9}$ samples. $b_{1}$ and $b_{2}$ refer to $b$ axis lattice parameters in the rock-salt layer $\mathrm{Ca}_{2} \mathrm{CoO}_{3}$ and the $\mathrm{CoO}_{2}$ layer, respectively.

\begin{tabular}{llllll}
\hline $\mathrm{Ca}_{3-\mathrm{x}} \mathrm{Tb}_{\mathrm{x}} \mathrm{Co}_{4} \mathrm{O}_{9}$ & $\mathrm{a}(\AA)$ & $\mathrm{b}_{1}(\AA)$ & $\mathrm{b}_{2}(\AA)$ & $\mathrm{c}(\AA)$ & $\beta\left(^{\circ}\right)$ \\
\hline$x=0$ & 4.859 & 4.562 & 2.747 & 10.849 & 98.711 \\
$x=0.05$ & 4.858 & 4.552 & 2.749 & 10.847 & 98.697 \\
$x=0.30$ & 4.858 & 4.551 & 2.750 & 10.840 & 98.536 \\
$x=0.50$ & 4.847 & 4.540 & 2.753 & 10.810 & 98.378 \\
\hline
\end{tabular}

SEM images of $\mathrm{Ca}_{3-x} \mathrm{~Tb}_{x} \mathrm{Co}_{4} \mathrm{O}_{9}$ samples after the sintering stage are shown in Figure 7-4. The plan-view SEM images in Figure 7-4 (a)-(d) clearly depict the size of the crystals progressively decreasing with the increase in the $\mathrm{Tb}$ doping level in $\mathrm{Ca}_{3-x} \mathrm{~Tb}_{x} \mathrm{Co}_{4} \mathrm{O}_{9}$ samples. Figure 7-4 (e)-(h) shows the cross-sectional SEM images taken from the fractured surface of the samples, it is evident that all doping levels present large anisotropy with micron-sized plate-shaped grains having the larger dimension in direction to the $a-b$ plane of the monoclinic $\mathrm{Ca}_{3} \mathrm{Co}_{4} \mathrm{O}_{9}{ }^{[11]}$. The arrow in each cross-sectional SEM image indicates the pressing direction of the pellet. The increasing Tb doping 

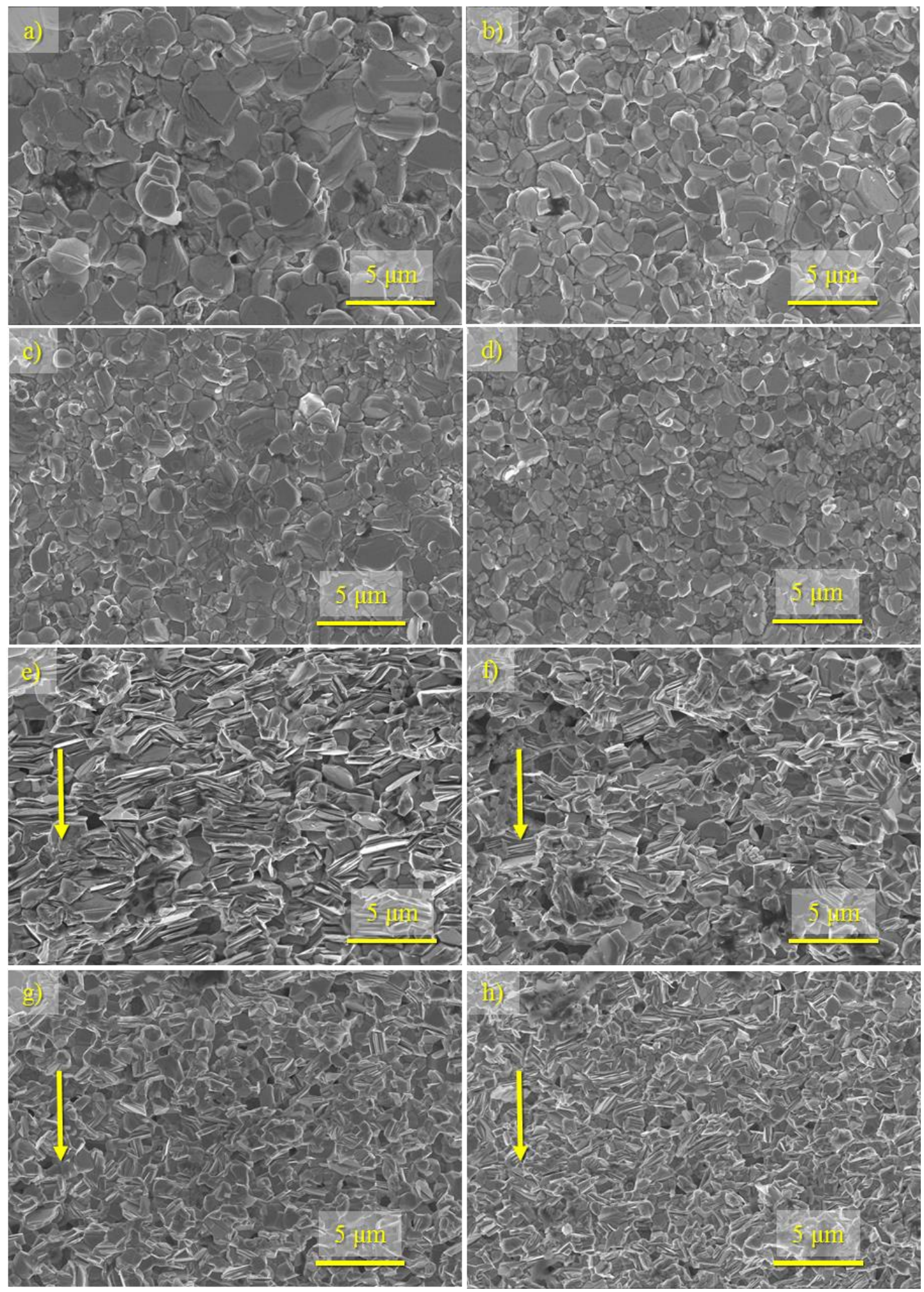

Figure 7-4: SEM images of plan-view [a)-d)] and cross-section of [e)-f)] $\mathrm{Ca}_{3-x} \mathrm{~Tb}_{x} \mathrm{Co}_{4} \mathrm{O}_{9}$ samples after sintering stage. a) and e) $\mathrm{Ca}_{3} \mathrm{Co}_{4} \mathrm{O}_{9}$, b) and f) $\mathrm{Ca}_{2.95} \mathrm{~Tb}_{0.05} \mathrm{Co}_{4} \mathrm{O}_{9}$, c) and g) $\mathrm{Ca}_{2.7} \mathrm{~Tb}_{0.3} \mathrm{Co}_{4} \mathrm{O}_{9}$, d) and h) $\mathrm{Ca}_{2.5} \mathrm{~Tb}_{0.5} \mathrm{Co}_{4} \mathrm{O}_{9}$. Arrows represent pressing direction of samples. 
level deteriorates the crystal texture and produce a more arbitrary crystal grain orientation as shown in Figure 7-4 (e)-(h). Additionally, SEM was taken from the calcined powders of the undoped $\mathrm{Ca}_{3} \mathrm{Co}_{4} \mathrm{O}_{9}$ and the $\mathrm{Ca}_{2.5} \mathrm{~Tb}_{0.5} \mathrm{Co}_{4} \mathrm{O}_{9}$ samples (Figure 7-5). This corroborates that the decrease in grain size is existing even before pressing and sintering the doped samples.
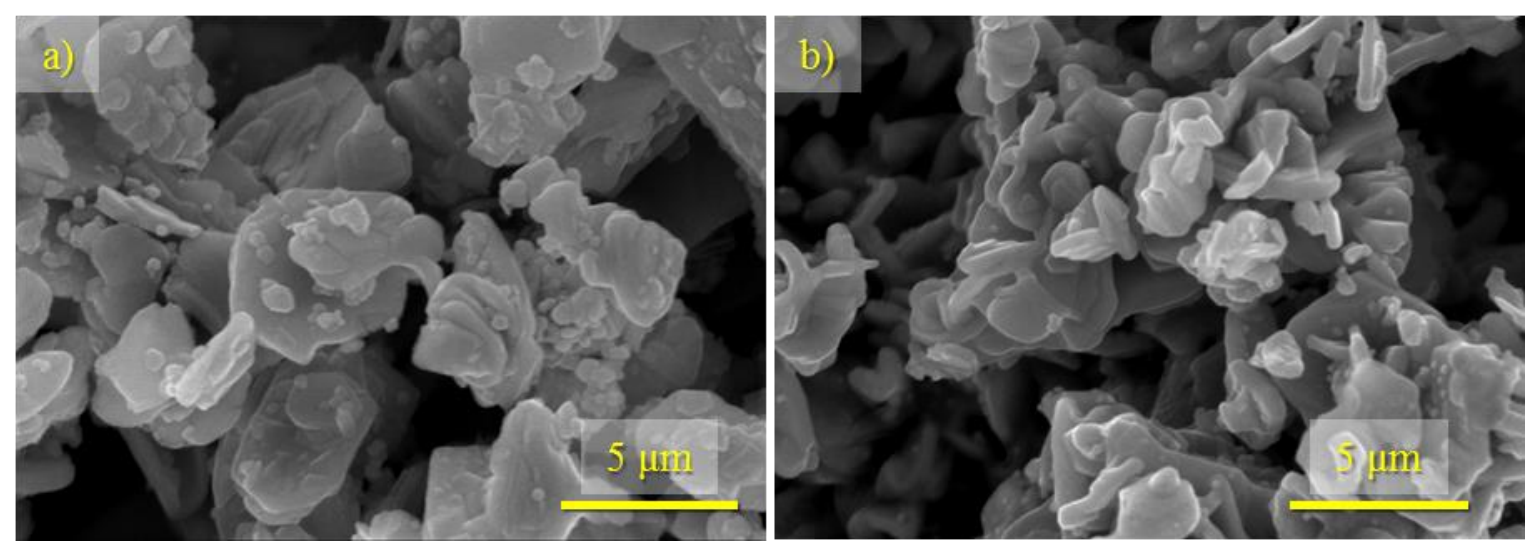

Figure 7-5: SEM images of the undoped (a) $\mathrm{Ca}_{3} \mathrm{CO}_{4} \mathrm{O}_{9}$ and the doped (b) $\mathrm{Ca}_{2.5} \mathrm{~Tb}_{0.5} \mathrm{CO}_{4} \mathrm{O}_{9}$ calcined powders.

TEM examination was taken to examine the effect of the $\mathrm{Tb}$ doping on the nanostructure of the calcium cobaltite ceramics. The TEM was performed on the single doped $\mathrm{Ca}_{2.5} \mathrm{~Tb}_{0.5} \mathrm{Co}_{4} \mathrm{O}_{9}$ sample. TEM images (shown in Figure 7-6) depict that each of the micron-sized crystal grains shown in the SEM images are conformed by a conglomeration of nanolamellas having different $c$ axes. The TEM image of the $\mathrm{Ca}_{2.5} \mathrm{~Tb}_{0.5} \mathrm{Co}_{4} \mathrm{O}_{9}$ in Figure 7-6 (b) illustrate the nanolamella structure from adjacent crystal grains having a large amount of high angle GBs between them, where the arrows are in parallel direction to the $c$-axes.

To explore the chemical changes of $\mathrm{Tb}$ as a single dopant EDS examination was recorded on the GBs and grain interior of $\mathrm{CCO}$ crystals to determine the atomic ratio of the different elements. The circle spots in Figure 7-6 (a) correspond to the location of EDS between the adjacent grains. The atomic ratio for each EDS spot is summarized in Table 7-3 for $\mathrm{Ca}_{2.5} \mathrm{~Tb}_{0.5} \mathrm{Co}_{4} \mathrm{O}_{9}$. 

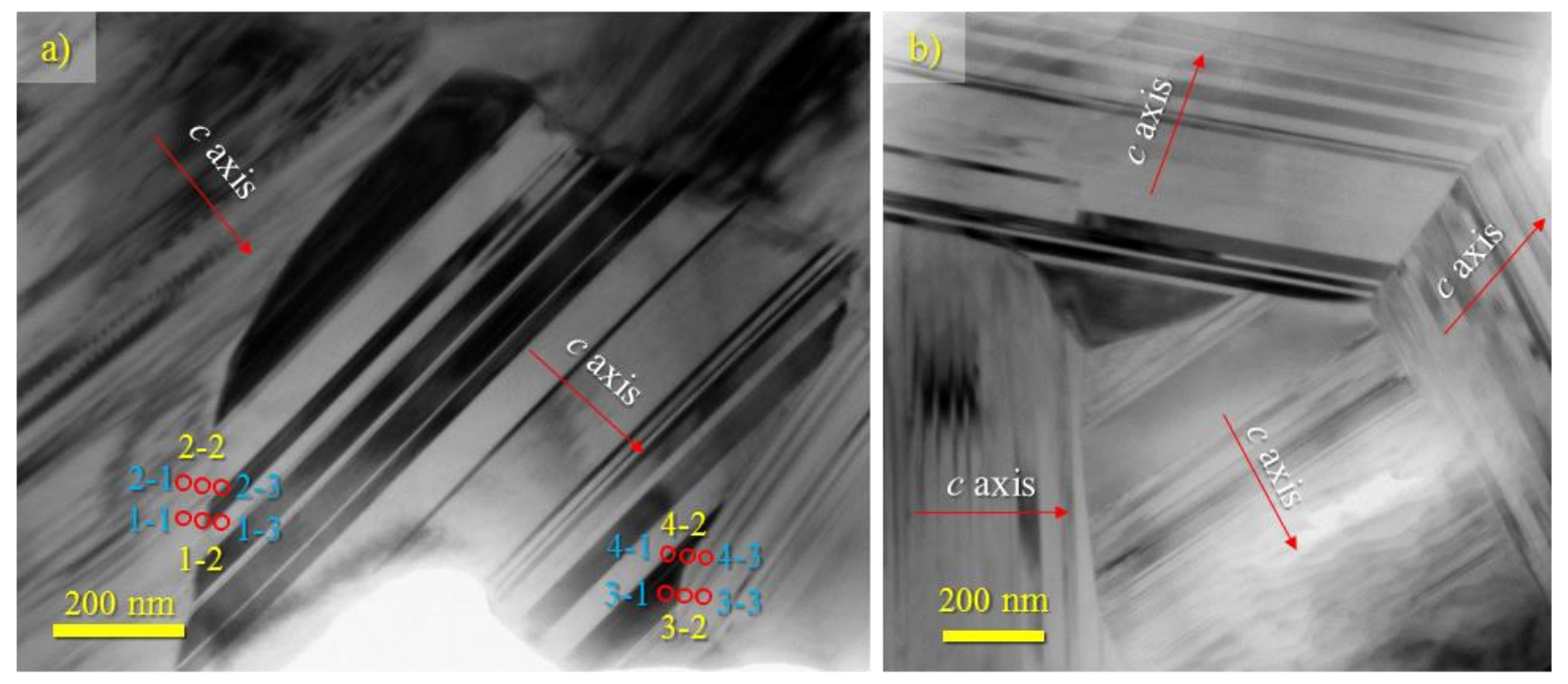

Figure 7-6: TEM images showing the nanostructure of the $\mathrm{Ca}_{2.5} \mathrm{~Tb}_{0.5} \mathrm{Co}_{4} \mathrm{O}_{9}$ sample. (a) The grain boundary from the crystals with different c-axis orientations. The red circles on the TEM images indicate the electron beam sample areas for the EDS data acquisition. (b) TEM showing that adjacent crystals have a large amount of high angle GBs between them.

As shown in Table 7-3, there is presence of $\mathrm{Tb}$ in both the crystal lattice and the GBs, however, there is a notably depletion in the GBs. Hence, EDS results are consistent with the information obtained by XRD analysis that showed the $\mathrm{Ca}_{3} \mathrm{Co}_{4} \mathrm{O}_{9}$ lattice is restrained by the incorporation of $\mathrm{Tb}$ in the doping material, even though there is depletion of $\mathrm{Tb}$ at the grain boundaries.

Table 7-3: Chemistry of the grain interior and grain boundaries of $\mathrm{Ca}_{2.5} \mathrm{~Tb}_{0.5} \mathrm{Co}_{4} \mathrm{O}_{9}$ for different sites labeled in the TEM image in Figure 7-6(a).

\begin{tabular}{lcccccc}
\hline $\mathrm{At} \%$ & $1-1$ & $1-2$ & $1-3$ & $2-1$ & $2-2$ & $2-3$ \\
\hline $\mathrm{Ca}$ & 13.28 & 15.63 & 15.9 & 16.97 & 13.78 & 17.19 \\
$\mathrm{Co}$ & 17.26 & 16.18 & 14.23 & 15.28 & 16.69 & 15.16 \\
$\mathrm{O}$ & 69.04 & 67.79 & 69.30 & 67.31 & 69.15 & 67.14 \\
$\mathrm{~Tb}$ & 0.41 & 0.40 & 0.57 & 0.44 & 0.41 & 0.51 \\
\hline $\mathrm{At} \%$ & $3-1$ & $3-2$ & $3-3$ & $4-1$ & $4-2$ & $4-3$ \\
\hline $\mathrm{Ca}$ & 13.39 & 12.54 & 12.56 & 15.55 & 14.98 & 15.21 \\
$\mathrm{Co}$ & 15.23 & 16.60 & 15.73 & 17.08 & 17.35 & 17.08 \\
$\mathrm{O}$ & 71.06 & 70.58 & 71.32 & 66.92 & 67.31 & 66.52 \\
$\mathrm{~Tb}$ & 0.32 & 0.28 & 0.38 & 0.44 & 0.36 & 0.40 \\
\hline
\end{tabular}


Figure 7-7 shows the XPS results taken from $\mathrm{Ca}_{3-x} \mathrm{~Tb}_{x} \mathrm{Co}_{4} \mathrm{O}_{9}$ samples after they were sintered. For the substitution of $\mathrm{Tb}$ into the $\mathrm{Ca}_{3} \mathrm{Co}_{4} \mathrm{O}_{9}$, there is a gradual shift to a lower binding energy of the Co $2 p_{3 / 2}$ and $2 p_{1 / 2}$ peaks from XPS spectrum with the increasing doping level. Additionally, the $\mathrm{Tb} 4 \mathrm{~d}$ XPS spectrum was taken for the highest doping level of $\mathrm{Ca}_{2.5} \mathrm{~Tb}_{0.5} \mathrm{Co}_{4} \mathrm{O}_{9}$ to understand the valence state of $\mathrm{Tb}$ while doping into the $\mathrm{CCO}$. A broad peak of the $4 \mathrm{~d}$ band was observed for the $\mathrm{Ca}_{2.5} \mathrm{~Tb}_{0.5} \mathrm{Co}_{4} \mathrm{O}_{9}$ samples, agreeing with reported literature of mixed valence state $\mathrm{Tb}^{[10]}$

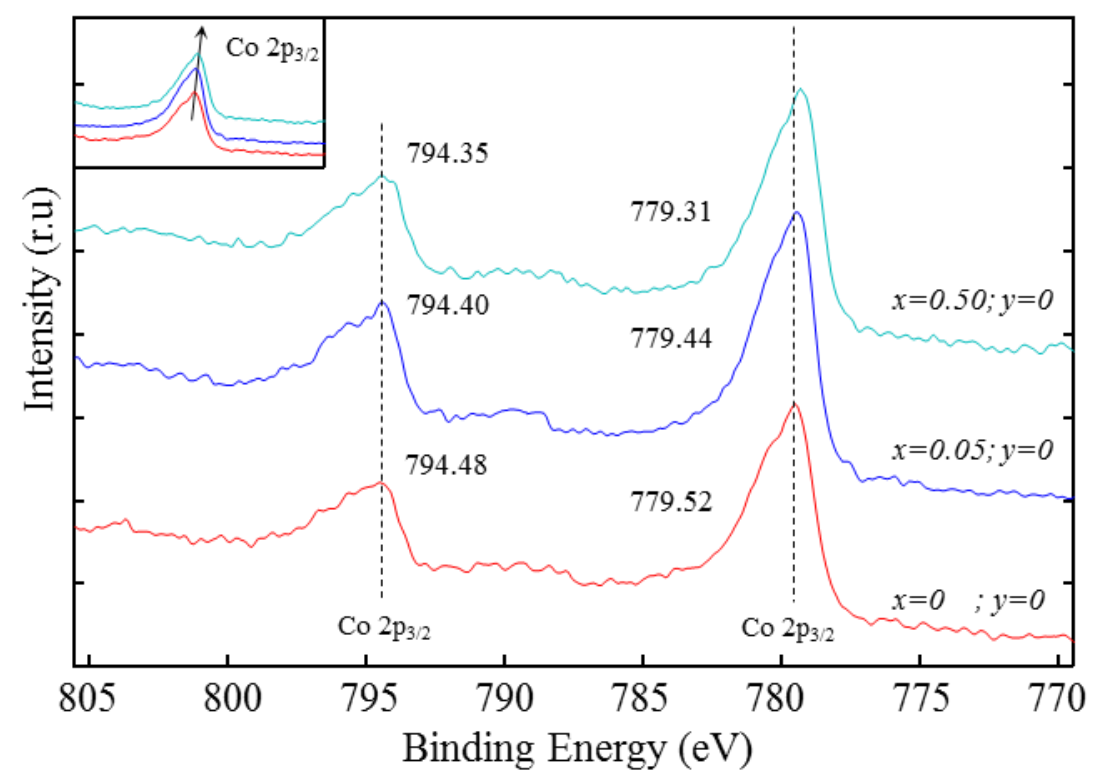

Figure 7-7: $\mathrm{Co} 2 p_{3 / 2}$ and $2 p_{1 / 2}$ peaks from XPS spectrum of $\mathrm{Ca}_{3-x} \mathrm{~Tb}_{x} \mathrm{Co}_{4} \mathrm{O}_{9}$ samples. Inset figure showing Co $2 p_{3 / 2}$ peaks shifting to a lower binding energy.

To further analyze the XPS results, the deconvolution of Co 2p $\mathrm{p}_{3 / 2}$ peak from XPS spectrums the different oxidation states of Co is presented in Figure 7-8. It is shown that the Co ions have three oxidation states: $\mathrm{Co}^{2+}, \mathrm{Co}^{3+}$ and $\mathrm{Co}^{4+}$ in all samples. Table 7-4 shows the percentage of each oxidation states and the $\mathrm{Co}^{4+} / \mathrm{Co}^{3}$ ratio in the analyzed samples. $\mathrm{Co}^{3+} / \mathrm{Co}^{4+}$ is mostly associated with the $\mathrm{CoO}_{2}$ hexagonal layers and $\mathrm{Co}^{2+} / \mathrm{Co}^{3+}$ is associated with the $\mathrm{Ca}_{2} \mathrm{CoO}_{3}$ rock-salt layers. Therefore, reducing the $\mathrm{Co}^{4+} / \mathrm{Co}^{3}$ ratio in the bulk material could lead to an improved the thermopower. 

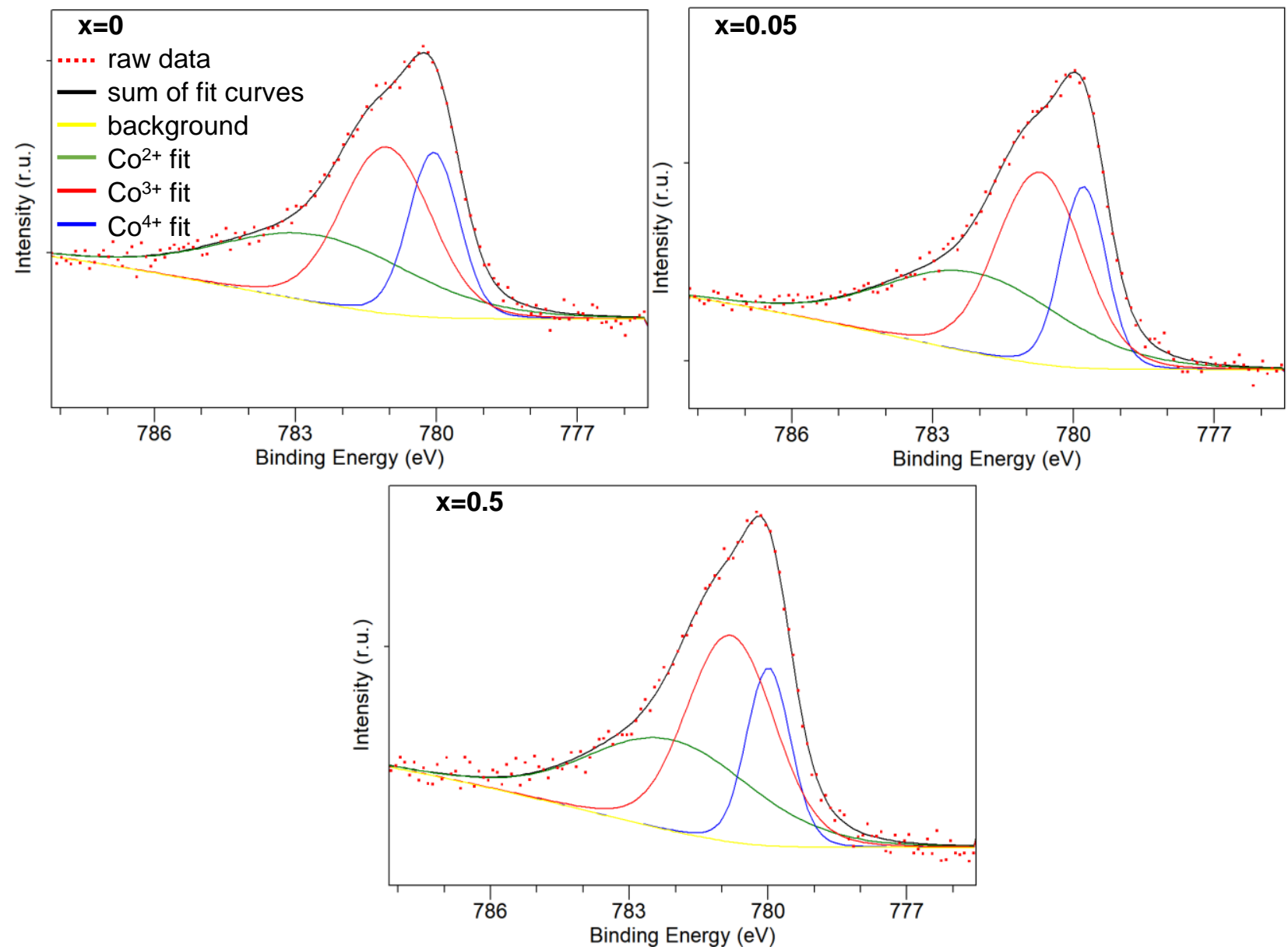

Figure 7-8: Deconvolution of $\mathrm{Co} 2 p_{3 / 2}$ peak from XPS spectrums of $\mathrm{Ca}_{3-x} \mathrm{~Tb}_{x} \mathrm{Co}_{4} \mathrm{O}_{9}$ samples.

Table 7-4: Ratios of $\mathrm{Co}^{4+}, \mathrm{Co}^{3+}$ and $\mathrm{Co}^{2+}$ from the XPS deconvolution of the Co 2p3/2 peak.

\begin{tabular}{lcccc}
\hline $\mathrm{Ca}_{3-\mathrm{x}} \mathrm{Tb}_{\mathrm{x}} \mathrm{Co}_{4} \mathrm{O}_{9}$ & $\mathrm{Co}^{4+}(\%)$ & $\mathrm{Co}^{3+}(\%)$ & $\mathrm{Co}^{2+}(\%)$ & $\mathrm{Co}^{4+} / \mathrm{Co}^{3+}(\%)$ \\
\hline$x=0 \quad ; y=0$ & 23.06 & 35.30 & 41.64 & 0.653 \\
$x=0.05 ; y=0$ & 20.99 & 43.01 & 36.00 & 0.488 \\
$x=0.50 ; y=0$ & 18.96 & 45.41 & 35.63 & 0.417 \\
\hline
\end{tabular}

\section{5 Discussion}

Substitution of $\mathrm{Ca}$ ions by $\mathrm{Tb}$ ions in polycrystalline $\mathrm{Ca}_{3} \mathrm{Co}_{4} \mathrm{O}_{9}$ ceramics is incorporated in the crystal lattice, as has been demonstrated in previous reports ${ }^{[10]}$. In our material, the incorporation of $\mathrm{Tb}$ into the crystal lattice can be confirmed since the difference in the ionic radius produces a slight shift to a higher Bragg's angle of the XRD peaks. The decrease in the lattice parameter 
values of $a, b_{1}, c$, and $\beta$ is a direct consequence of the dopant incorporation into the material. The reduction of the lattice is caused by replacing the $\mathrm{Ca}^{2+}$ sites by smaller $\mathrm{Tb}$ ions, suggesting that $\mathrm{Tb}$ atoms are indeed incorporated in place of Ca lattice sites.

As SEM and TEM showed, Tb reduces considerably the size of the $\mathrm{Ca}_{3} \mathrm{Co}_{4} \mathrm{O}_{9}$ ceramics, but particularly generates a more arbitrary orientation of the crystal grains in both the micron sized crystals and in the nanollamelas conforming such crystals. These microstructure features are directly linked to the carrier mobility ${ }^{[16,17]}$. The electrical conductivity could be expressed as $\sigma \equiv n e \mu$, where $\mu$ is the carrier mobility, $e$ is the elementary positive charge constant, and $n$ is the number density of electrons, thus, the increased of $\rho$ in ceramics is caused from both poor carrier mobility and the reduction of carrier concentration. This yields a larger $\rho$ in the $\mathrm{Ca}_{3-\mathrm{x}} \mathrm{Tb}_{\mathrm{x}} \mathrm{Co}_{4} \mathrm{O}_{9}$ ceramics and a decrease in the PF performance.

The substantial increase in the $\mathrm{S}$ values from the doped samples infers a decrease in the carrier concentration of the $\mathrm{Ca}_{3} \mathrm{Co}_{4} \mathrm{O}_{9}$ ceramics. At high temperature $(T \rightarrow \infty)$ the Seebeck coefficient of the $\mathrm{Ca}_{3} \mathrm{Co}_{4} \mathrm{O}_{9}$ can be expressed with Heike's formula as ${ }^{[18]}$ :

$$
S_{(T \rightarrow \infty)}=-\frac{k_{B}}{e} \ln \left(\frac{g_{3}}{g_{4}} \frac{x}{1-x}\right)
$$

where $\mathrm{g}_{3}$ and $\mathrm{g}_{4}$ is the number of configuration of the $\mathrm{Co}^{3+}$ and $\mathrm{Co}^{4+}$ ions, respectively, and $\mathrm{x}$ is the fraction of $\mathrm{Co}^{4+}$ in the $\mathrm{CoO}_{2}$ layer. $k_{B}$ and $e$ are the Boltzmann constant and the electronic charge, respectively. It can be expected that the magnitude of $S$ increase with a reduction of $\mathrm{Co}^{4+}$ concentration. Additionally it has been reported that the segregation at the GBs acts as a barrier layer mechanism in bulk semiconductor originated by a band offset among the grain boundary and the grain interior ${ }^{[19]}$. For the single dopant of $\mathrm{Tb}$, there is a gradual shift to a lower binding energy for the Co $2 \mathrm{p}_{3 / 2}$ and $2 \mathrm{p}_{1 / 2}$ peaks from XPS spectrum due to the substitution of $\mathrm{Ca}^{2+}$ by superior valance $\mathrm{Tb}$ ions, where the mixed valence state of $\mathrm{Tb}^{3+} / \mathrm{Tb}^{4+}$ plays an important role in the 
reduction of $\mathrm{Co}^{4+}$ concentration, and thus, the reduction of carrier concentration, this is consistent with previous studies in Tb doped CCO. ${ }^{[10]}$

The total $\kappa$ in $\mathrm{Ca}_{3} \mathrm{Co}_{4} \mathrm{O}_{9}$ is a cumulative effect of the electronic thermal conductivity $\left(\kappa_{e}\right)$ and the lattice thermal conductivity $\left(\kappa_{i}\right)$. Nevertheless, $\mathrm{Ca}_{3} \mathrm{Co}_{4} \mathrm{O}_{9}$ ceramics predominant component is the contribution of $\kappa_{i}$. Improvements in the electronic transport properties, leads to an increase in the $\kappa_{e}$ component. Still, $\kappa_{e}$ possess a less significant impact on the overall $\kappa$. Tb replaces the host ion $\mathrm{Ca}$ in $\mathrm{Ca}_{3} \mathrm{Co}_{4} \mathrm{O}_{9}$, but it is much heavier in atomic mass. Thus, introducing a heavier atom in the lattice site unquestionably intensifies the phonon scattering events. The scattering is attributed to the difference in atomic mass in the ions. Heavier ions vibrate easily from their neighboring light ions which cause local vibration that result in a shortened phonon mean free path $^{[20]}$. Moreover, the increased grain boundary density in the material can also play a factor in the scattering events of the material. $\mathrm{Tb}$ deteriorates the electronic transport properties, which causes $\kappa_{e}$ to decrease. Thus, the inclusion of $\mathrm{Tb}$ decreases gradually the overall $\kappa$ with the increasing doping level.

Overall, the increase of $S$ coupled with an almost unchanged $\rho$ values and the reduction of $\kappa$ in $\mathrm{Ca}_{2.95} \mathrm{~Tb}_{0.05} \mathrm{Co}_{4} \mathrm{O}_{9}$ materials produced a subtle increase in the $Z T$ up to 0.38 at $1073 \mathrm{~K}$ compared to the value of 0.35 for pristine $\mathrm{Ca}_{3} \mathrm{Co}_{4} \mathrm{O}_{9}$. Moreover, carrier mobility and grain alignment(to further improve $\rho$ ), selective carrier filtering(to further improve $S$ ), and higher concentration of heavier atoms(to further reduce $\kappa$ ) could be possible with the inclusion of a second dopant to promote both the nanostructure and the TE properties with previous reported elements such as $\mathrm{Bi}^{[21]}$ and $\mathrm{K}^{[22]}$. 


\section{6 Conclusion}

The improvement of TE performance of polycrystalline $\mathrm{Ca}_{3} \mathrm{CO}_{4} \mathrm{O}_{9}$ by the substitution of $\mathrm{Tb}$ was shown in this chapter. The optimal concentration $\mathrm{Ca}_{2.95} \mathrm{~Tb}_{0.05} \mathrm{Co}_{4} \mathrm{O}_{9}$ simultaneously reduce the $\kappa$ and increase of Seebeck coefficient which lead to a subtle increase of the $Z T$ value to 0.38 , which is a $10 \%$ improvement compared to CCO baseline. The lowest $\kappa$ achieved is $25 \%$ lower than the baseline material, while the peak value of $S$ improvement represents a $45 \%$. Both $S$ and $\kappa$ showed how big influence the rare earth element dopants have into $\mathrm{Ca}_{3} \mathrm{Co}_{4} \mathrm{O}_{9}$. Additionally, TEM results showed evidence of $\mathrm{Tb}$ at the crystal lattice and XRD confirmed a decrease in the lattice parameter of the unit cell due to $\mathrm{Tb}$ smaller size. TEM results also showed the depletion of $\mathrm{Tb}$ at the GBs. Nevertheless, the biggest importance in Tb doping is the ability to reduce the crystal size of the material and at the same time vastly improve $S . \kappa$ is suppressed by the increased scattering events of the heavier $\mathrm{Tb}$ atoms incorporated into the $\mathrm{CCO}$ and the larger grain boundary density. The lowest value achieved for $\mathrm{Ca}_{2.5} \mathrm{~Tb}_{0.5} \mathrm{Co}_{4} \mathrm{O}_{9} \sim 1.28 \mathrm{~W} / \mathrm{mK}$ represents a favorably $\sim 24 \%$ reduction in the thermal transport properties of the bulk ceramic. The optimal $\mathrm{Tb}$ doped $\mathrm{CCO}$ with $10 \%$ improvement on the peak ZT value could be the starting point to further improve CCO by the addition of a second dopant to control nanostructure and the TE properties, in order to attain better electrical properties. 


\section{References}

1. Forman, C., et al., Estimating the global waste heat potential. Renewable and Sustainable Energy Reviews, 2016. 57: p. 1568-1579.

2. Snyder, G.J. and E.S. Toberer, Complex thermoelectric materials. Nature Materials, 2008. 7: p. 105.

3. Kim, S.I., et al., Dense dislocation arrays embedded in grain boundaries for highperformance bulk thermoelectrics. Science, 2015. 348(6230): p. 109.

4. Joshi, G., et al., Enhanced Thermoelectric Figure-of-Merit in Nanostructured p-type Silicon Germanium Bulk Alloys. Nano Letters, 2008. 8(12): p. 4670-4674.

5. Koumoto, K., et al., Thermoelectric Ceramics for Energy Harvesting. Journal of the American Ceramic Society, 2013. 96(1): p. 1-23.

6. Woermann, E. and A. Muan, Phase equilibria in the system CaO-cobalt oxide in air. Journal of Inorganic and Nuclear Chemistry, 1970. 32(5): p. 1455-1459.

7. LeBlanc, S., et al., Material and manufacturing cost considerations for thermoelectrics. Renewable and Sustainable Energy Reviews, 2014. 32: p. 313-327.

8. Shikano, M. and R. Funahashi, Electrical and thermal properties of single-crystalline $\left(\mathrm{Ca}_{2} \mathrm{CoO}_{3}\right)_{0.7} \mathrm{CoO}_{2}$ with a $\mathrm{Ca}_{3} \mathrm{Co}_{4} \mathrm{O}_{9}$ structure. Applied Physics Letters, 2003. 82(12): p. 1851-1853.

9. Fergus, J.W., Oxide materials for high temperature thermoelectric energy conversion. Journal of the European Ceramic Society, 2012. 32(3): p. 525-540.

10. Saini, S., et al., Terbium Ion Doping in $\mathrm{Ca}_{3} \mathrm{Co}_{4} \mathrm{O}_{9}$ : A Step towards High-Performance Thermoelectric Materials. Scientific Reports, 2017. 7: p. 44621.

11. Chen, S., et al., Effect of precursor calcination temperature on the microstructure and thermoelectric properties of $\mathrm{Ca}_{3} \mathrm{Co}_{4} \mathrm{O}_{9}$ ceramics. Journal of Sol-Gel Science and Technology, 2012. 64(3): p. 627-636.

12. Boyle, C., et al., Improving the thermoelectric performance and thermal stability of $\mathrm{Ca}_{3} \mathrm{Co}_{4} \mathrm{O}_{9+\delta}$ ceramics by sintering in oxygen atmosphere. Journal of Sol-Gel Science and Technology, 2018. 85(3): p. 712-722.

13. Sotelo, A., et al., Improvement of thermoelectric properties of $\mathrm{Ca}_{3} \mathrm{Co}_{4} \mathrm{O}_{9}$ using soft chemistry synthetic methods. Journal of the European Ceramic Society, 2012. 32(10): p. 2415-2422.

14. Delorme, F., et al., Thermoelectric properties of $\mathrm{Ca}_{3} \mathrm{CO}_{4} \mathrm{O}_{9}-\mathrm{Co}_{3} \mathrm{O}_{4}$ composites. Ceramics International, 2015. 41(8): p. 10038-10043.

15. Boyle, C., et al., Competing dopants grain boundary segregation and resultant seebeck coefficient and power factor enhancement of thermoelectric calcium cobaltite ceramics. Ceramics International, 2017. 43(14): p. 11523-11528.

16. Mikami, M., et al., Enhancement of Electrical Properties of the Thermoelectric Compound $\mathrm{Ca}_{3} \mathrm{Co}_{4} \mathrm{O}_{9}$ through Use of Large-grained Powder. Journal of Materials Research, 2011. 20(9): p. 2491-2497.

17. Guilmeau, E., et al., Thermoelectric properties-texture relationship in highly oriented $\mathrm{Ca}_{3} \mathrm{Co}_{4} \mathrm{O}_{9}$ composites. Applied Physics Letters, 2004. 85(9): p. 1490-1492.

18. Cutler, M. and N.F. Mott, Observation of Anderson Localization in an Electron Gas. Physical Review, 1969. 181(3): p. 1336-1340. 
19. Mehdizadeh Dehkordi, A., et al., Large Thermoelectric Power Factor in Pr-Doped $\mathrm{SrTiO}_{3-\delta}$ Ceramics via Grain-Boundary-Induced Mobility Enhancement. Chemistry of Materials, 2014. 26(7): p. 2478-2485.

20. Wang, Y., et al., Doping-Induced Metal-Insulator Transition and the Thermal Transport Properties in $\mathrm{Ca}_{3-x} \mathrm{Y}_{x} \mathrm{Co}_{4} \mathrm{O}_{9}$. The Journal of Physical Chemistry C, 2010. 114(11): p. 51745181.

21. Boyle, C., et al., Grain boundary segregation and thermoelectric performance enhancement of bismuth doped calcium cobaltite. Journal of the European Ceramic Society, 2016. 36(3): p. 601-607.

22. Romo-De-La-Cruz, C., et al., Role of oversized dopant potassium on the nanostructure and thermoelectric performance of calcium cobaltite ceramics. Sustainable Energy \& Fuels, 2018. 2(4): p. 876-881. 


\section{Chapter 8. Impact of Potassium Non-Stoichiometric Addition and Terbium Stoichiometric Substitution on the Thermoelectric Performance of $\mathrm{Ca}_{3} \mathrm{Co}_{4} \mathrm{O}_{9}$ Ceramics}

\section{1 Different size of dopants for better ZT performance}

The influence of a dual doping approach using the non-stoichiometric addition of isovalent Potassium and multivalent Terbium is investigated. The dual doping approach develops an enhanced microstructure and thermoelectric performance of the misfit layered calcium cobaltite $\mathrm{Ca}_{3} \mathrm{Co}_{4} \mathrm{O}_{9}$ ceramics. The undoped polycrystalline $\mathrm{Ca}_{3} \mathrm{Co}_{4} \mathrm{O}_{9}$ and 4 different doping concentrations with the designed nominal chemistry of $\mathrm{Ca}_{3-\mathrm{x}} \mathrm{Tb}_{\mathrm{x}} \mathrm{Co}_{4} \mathrm{O}_{9} \mathrm{~K}_{y}(x=0.05, \mathrm{y}=0 ; x=0.05, \mathrm{y}=0.1 ; x=0.5$, $\mathrm{y}=0 ; x=0.5, \mathrm{y}=0.1)$ are explored in the present work. The inclusion of the different sized dopants simultaneously promoted the electrical and thermal properties while promoting crystal texture and grain alignment. TEM observation showed the grain boundary segregation of the oversized Potassium whereas Terbium was present in both the lattice and in the grain boundary. XRD results further confirmed the TEM observations and provide evidence of a mostly single phase ceramics. The figure of merit $Z T$ of the material with the dual doping approach is improved to a value of 0.47 at $1073 \mathrm{~K}$ achieved by $\mathrm{Ca}_{2.95} \mathrm{~Tb}_{0.05} \mathrm{Co}_{4} \mathrm{O}_{9} \mathrm{~K}_{0.1}$, such value represents a $10 \%$ enhancement in relation to the best performing calcium cobaltite of each independent doping element (ZT 0.42 and $\mathrm{ZT} \sim 0.37$ for $\mathrm{Ca}_{3} \mathrm{Co}_{4} \mathrm{O}_{9} \mathrm{~K}_{0.1}$ and $\mathrm{Ca}_{2.95} \mathrm{~Tb}_{0.05} \mathrm{Co}_{4} \mathrm{O}_{9}$ respectively). Furthermore, the improved values of ZT for the dual doping material drives the study to further examine dual doping systems to enhance the performance of the $\mathrm{Ca}_{3} \mathrm{Co}_{4} \mathrm{O}_{9}$ ceramics. In the present work, the impact of the dual doping approach by $\mathrm{Tb}$ and $\mathrm{K}$ on the microstructure and thermoelectric properties of the $\mathrm{Ca}_{3} \mathrm{Co}_{4} \mathrm{O}_{9}$ ceramics is further discussed. 


\section{2 Introduction}

Worldwide energy consumption losses more than $60 \%$ of the energy after its conversion, ${ }^{[1]}$ most of these losses are in the form of waste heat. Therefore, it is a matter of utmost importance to harness waste heat and reutilize this energy source to improve the overall conversion efficiency. Thermoelectric (TE) technology is one of the rising alternatives to harvest the excessive amount of energy lost as waste heat for a better and sustainable energy future ${ }^{[2]}$. TE materials can utilize waste heat to improve efficiency in the ever-increasing demand for energy by converting a temperature difference directly into electricity due to the Seebeck effect ${ }^{[3]}$. Such TE devices can be applied to provide an active cooling system for electronic devices, to produce energy in extreme environments, to generate electric power in remote areas and to power autonomously microsensors. ${ }^{[4]}$ Additionally, the recent widespread adoption of green technologies could facilitate to introduce the domestic and industrial applications of thermoelectric generators due to its emphasis as a renewable energy source.

The energy conversion efficiency of the TE materials is described by the figure of merit $Z T$, which is defined as $Z T=S^{2} \rho^{-1} \kappa^{-1} T$, where $S, \rho, S^{2} \rho^{-1}$, and $\kappa$ are the Seebeck coefficient, electrical resistivity, electrical power factor(PF) and thermal conductivity, respectively ${ }^{[5]}$. Conventional materials have shown a high potential due to its ZT above the unit ${ }^{[6,7]}$. Nonetheless, such conventional TE compounds are comprised with elements such as $\mathrm{Te}, \mathrm{Sb}$, and $\mathrm{Ge}$, that can be oxidized if exposed to the high temperature air ${ }^{[8]}$. Alternatively, TE oxide materials are known to benefit from their earth abundancy, low cost, non-toxicity, and high thermal stability ${ }^{[8]}$. The $p$-type calcium cobaltite $\mathrm{Ca}_{3} \mathrm{Co}_{4} \mathrm{O}_{9}$ has gained a lot of attraction due to its excellent TE performance with a high $Z T$ value for the single crystal material ${ }^{[9],}$ approaching that of the well-developed conventional TE materials. Despite their single crystal performance, the TE energy conversion 
efficiency of polycrystalline $\mathrm{Ca}_{3} \mathrm{Co}_{4} \mathrm{O}_{9}$ remains low and accounts to $\sim 30-60 \%$ of the single crystal value $^{[10]}$, thus, a challenge remains to improve the bulk $\mathrm{Ca}_{3} \mathrm{Co}_{4} \mathrm{O}_{9}$.

Ion doping has been widely utilized to synthesize bulk thermoelectric materials with enhanced properties. Most importantly, stoichiometric substitution by rare earth elements have been shown to be able to separate the connection of the TE physical properties $(S, \rho$, and $\kappa$ interact with each other by the carrier concentration) to enhance the conversion efficiency of polycrystalline $\mathrm{Ca}_{3} \mathrm{Co}_{4} \mathrm{O}_{9}{ }^{[11-14]}$.

Another effective and novel strategy to overcome the limiting conditions in the polycrystalline $\mathrm{Ca}_{3} \mathrm{Co}_{4} \mathrm{O}_{9}$ is by controlling and modifying the grain boundary of neighboring crystals with the accurate selection of doping elements. Grain boundary engineering has been reported to have an important influence on the properties optimization on different TE materials ${ }^{[6,15-23]}$. Specifically, the segregation of doping elements into the $\mathrm{Ca}_{3} \mathrm{Co}_{4} \mathrm{O}_{9}$ has shown a drastic improve on the microstructure and electronic properties ${ }^{[24-27]}$. In particular, $\mathrm{K}^{+}$doped $\mathrm{Ca}_{3} \mathrm{Co}_{4} \mathrm{O}_{9}{ }^{[27]}$ resulted in a large PF and better texture of the bulk material. Consequently, to capitalize the effect of both techniques in order to maximize the energy conversion efficiency of polycrystalline $\mathrm{Ca}_{3} \mathrm{Co}_{4} \mathrm{O}_{9}$, a better understanding of the effects on grain boundary engineering with simultaneous ion doping is critical. Therefore, the effective selection of these rare earth dopants and the doping strategies to achieve a high performance for dual doped materials is essential to further expand these doping techniques in the bulk $\mathrm{Ca}_{3} \mathrm{Co}_{4} \mathrm{O}_{9}$.

This work shows that terbium and potassium were introduced through both the cation stoichiometric substitution and novel non-stoichiometric addition resulting in a dramatically decrease in the electrical resistivity, a simultaneously increase the Seebeck coefficient, and ultimately result in the polycrystalline ceramics improvement over the pristine $\mathrm{Ca}_{3} \mathrm{Co}_{4} \mathrm{O}_{9}$ ceramics. 
To achieve the best performance, the optimal synthesis conditions ${ }^{[28-30]}$ were employed to mitigate the possible formation of $\mathrm{Co}_{3} \mathrm{O}_{4}$ which affect the performance of $\mathrm{Ca}_{3} \mathrm{Co}_{4} \mathrm{O}_{9}$ ceramics. ${ }^{[31]}$

\section{3 Experimental section}

The polycrystalline ceramic samples $\mathrm{Ca}_{3} \mathrm{Co}_{4} \mathrm{O}_{9}$ and $\mathrm{Ca}_{3-\mathrm{x}} \mathrm{Tb}_{\mathrm{x}} \mathrm{Co}_{4} \mathrm{O}_{9} \mathrm{~K}_{y}(x=0.05, \mathrm{y}=0 ; x=0.05$, $\mathrm{y}=0.1 ; x=0.5, \mathrm{y}=0 ; x=0.5, \mathrm{y}=0.1)$ were synthesized by the conventional chemical sol-gel route[29]. The precursor powders were obtained mixing the chemical reagents according to the stoichiometric ratios. $\mathrm{Ca}\left(\mathrm{NO}_{3}\right)_{2} \cdot 4 \mathrm{H}_{2} \mathrm{O}$ (99\%, Acros Organics), $\mathrm{Co}\left(\mathrm{NO}_{3}\right){ }_{2} \cdot 6 \mathrm{H}_{2} \mathrm{O}$ (99\%, Acros Organics), $\mathrm{Tb}\left(\mathrm{NO}_{3}\right)_{3} \cdot 6 \mathrm{H}_{2} \mathrm{O}$ (99.9\%, Strem Chemicals), and $\mathrm{KNO}_{3}$ (99\%, Alfa Aesar) were dissolved in deionized water. Citric acid (BDH Chemical), ethylene glycol, and polyethylene glycol were used to polymerize the mixture, while nitric acid was added to the solution to facilitate the sol-gel synthesis, nitrate salts decomposition, and the new compound formation. The mixture was mechanically stirred at $353 \mathrm{~K}$ for $3 \mathrm{~h}$. The obtained sol-gel solution was ashed at $773 \mathrm{~K}$ for 2 $\mathrm{hr}$ in air inside a box furnace. The resulting ashes were ball-milled in ethyl alcohol in a stage with zirconia balls and then dried at room temperature with a final manually ground stage after that. The fine ashes were calcined at $973 \mathrm{~K}$ for $4 \mathrm{~h}$ in a tube furnace with a constant oxygen flow ${ }^{[30]}$. The final precursor powders were uniaxially pressed into round pellets under a 1 Gpa pressure at room temperature. The bulk samples were sintered at temperature $1233 \mathrm{~K}$ in a tube furnace with a constant oxygen flow ${ }^{[28]}$ and then cut into rectangular pellets to obtain the final desired sample for either the electrical or thermal measurements. The apparent densities for all bulk samples are listed in Table 8-1. 
Table 8-1: Apparent density of the $\mathrm{Ca}_{3-x} \mathrm{~Tb}_{x} \mathrm{Co}_{4} \mathrm{O}_{9} \mathrm{~K}_{y}$ samples.

\begin{tabular}{lll}
\hline $\mathrm{Ca}_{3-\mathrm{x}} \mathrm{Tb}_{\mathrm{x}} \mathrm{Co}_{4} \mathrm{O}_{9} \mathrm{~K}_{\mathrm{y}}$ & Average Apparent density $\left(\mathrm{g} / \mathrm{cm}^{3}\right)$ \\
\hline $\mathrm{x}=0$ & $\mathrm{y}=0$ & 3.883 \\
$\mathrm{x}=0.05$ & $\mathrm{y}=0$ & 4.182 \\
$\mathrm{x}=0.05$ & $\mathrm{y}=0.1$ & 4.576 \\
$\mathrm{x}=0.50$ & $\mathrm{y}=0$ & 4.578 \\
$\mathrm{x}=0.50$ & $\mathrm{y}=0.1$ & 4.758 \\
\hline
\end{tabular}

The electrical resistivity $(\rho)$ and absolute Seebeck coefficient $(S)$ were measured by the fourterminal method using a Linseis LSR-1100 unit. The measurements were performed direction to the pressed direction from $323 \mathrm{~K}$ to $1073 \mathrm{~K}$ under a low pressure He environment. The thermal conductivity was calculated using the equation $\kappa=\lambda C_{p} \rho m$, where $C_{p}, \lambda$, and $m$ are the specific heat, thermal diffusivity, and mass density, respectively. The $C p$ and $\lambda$ values were obtained from 323 K to $1073 \mathrm{~K}$ using a Linseis Laser Flash Analyzer 1200. The measurements were performed perpendicular to the pressed direction under a low-pressure air environment. X-Ray powder Diffraction (XRD) analysis was performed using a PANatycal X'Pert Pro XRD unit for crystal phase and lattice parameter determination purposes. The ground powders from the sintered samples were used in the $\mathrm{XRD}$ analysis using $\mathrm{Cu} \mathrm{K}$-alpha radiation, $45 \mathrm{kV}$ tension and $40 \mathrm{~mA}$ current at room temperature. The cross-section morphology of the sintered pellets was observed using a Hitachi-S4700F Scanning Electron Microscope (SEM) unit. The samples for transmission electron microscopy (TEM) were prepared by mechanically polishing and ion milling in a liquidnitrogen-cooled holder. Electron diffraction, diffraction contrast, and high-resolution TEM imaging were performed in a JEM-2100 operated at $200 \mathrm{kV}$. The chemical analysis was carried out using energy dispersive spectroscopy (EDS) under TEM. 


\section{4 Results}

Figure 8-1 shows the influence of the electrical transport properties for samples with different concentration levels of $\mathrm{Tb}$ stoichiometric substitution with simultaneous non-stoichiometric addition of Potassium. As previously reported, Tb increases the electrical resistivity in the calcium cobaltite ceramics, however by adding a second dopant $\mathrm{K}^{+}$the electrical resistivity is considerably reduced. It is noteworthy that the $\rho$-T curves of the dual dopant samples follow the same behavior of the $\mathrm{Tb}$ counterpart. Additionally, although the doped samples display a higher $\rho$ at a low temperature regime compared to baseline, the best performing sample $\mathrm{Ca}_{2.95} \mathrm{~Tb}_{0.05} \mathrm{Co}_{4} \mathrm{O}_{9} \mathrm{~K}_{0.1}$ reaches a low $\rho$ value of $48.4 \mu \Omega \mathrm{m}$ at $1073 \mathrm{~K}$. Such value represents a $\sim 27 \%$ decrease compared to a $66.1 \mu \Omega \mathrm{m}$ at $1073 \mathrm{~K}$ for the pristine ceramic. More interesting, the extremely doped $\mathrm{Ca}_{2.5} \mathrm{~Tb}_{0.5} \mathrm{Co}_{4} \mathrm{O}_{9} \mathrm{~K}_{0.1}$ shows a comparable $\rho$ to the baseline with a value of $68.3 \mu \Omega \mathrm{m}$ at $1073 \mathrm{~K}$.

As shown in Figure 8-1 (b) and previously reported, Tb increases considerably the $S$ values with the increasing doping level. Similarly, by adding $\mathrm{K}^{+}$as second dopant the $S$ values are further increased for both dual doping concentrations at high temperature. $\mathrm{Ca}_{2.5} \mathrm{~Tb}_{0.5} \mathrm{Co}_{4} \mathrm{O}_{9} \mathrm{~K}_{0.1}$ samples show a $S \sim 210 \mu \mathrm{VK}^{-1}$ at $1073 \mathrm{~K}$, which is a very significant improvement over the value of undoped calcium cobaltite ceramics with a $S \sim 190 \mu \mathrm{VK}^{-1}$. Nonetheless, the biggest contribution to the $S$ improvement is due to the $\mathrm{Tb}$ incorporation into the $\mathrm{Ca}_{3} \mathrm{Co}_{4} \mathrm{O}_{9}$.

A large electrical PF is achieved by $\mathrm{Ca}_{2.95} \mathrm{~Tb}_{0.05} \mathrm{Co}_{4} \mathrm{O}_{9} \mathrm{~K}_{0.1}$ with a value of $0.80 \mathrm{mWm}^{-1} \mathrm{~K}^{-2}(0.77$ $\mathrm{mWm}^{-1} \mathrm{~K}^{-2}$ at $1073 \mathrm{~K}$ ) due to the combined enhancements in the $\rho$ and S. Such values represent a $40 \%$ increase at $1073 \mathrm{~K}$ compared to pristine $\mathrm{Ca}_{3} \mathrm{Co}_{4} \mathrm{O}_{9}$ ceramics. It should be noted that the concentration $\mathrm{Ca}_{2.5} \mathrm{~Tb}_{0.5} \mathrm{Co}_{4} \mathrm{O}_{9} \mathrm{~K}_{0.1}$ showed a $\sim 70 \%$ increase in electrical PF performance compared to $\mathrm{Ca}_{2.5} \mathrm{~Tb}_{0.5} \mathrm{Co}_{4} \mathrm{O}_{9}$. Nevertheless, at the low temperature regime the electrical PF does not increase significantly due to the high $\rho$ in the samples at the $300 \mathrm{~K}$ to $600 \mathrm{~K}$ range. 

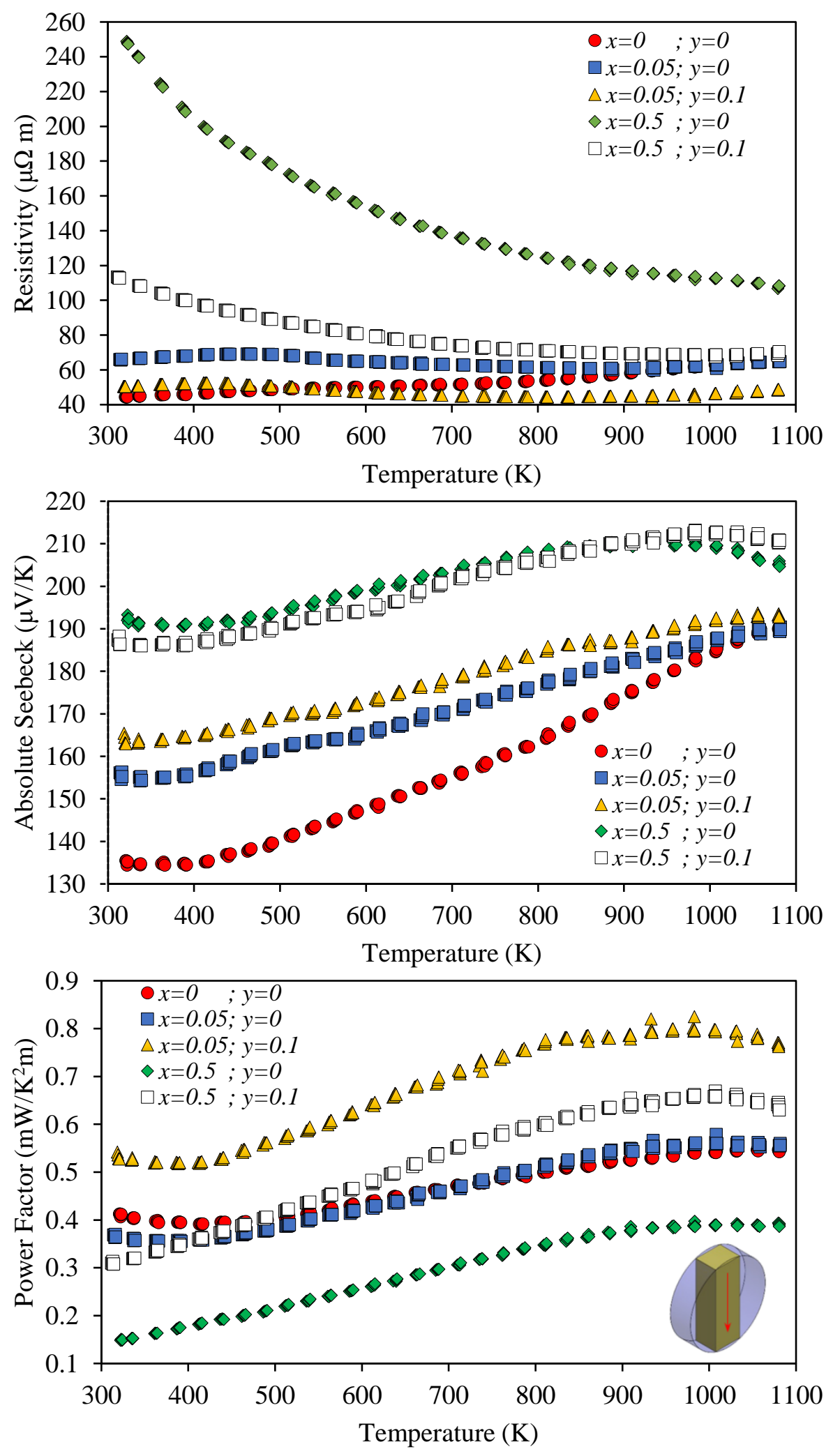

Figure 8-1: Temperature dependence of electrical properties for $\mathrm{Ca}_{3-x} \mathrm{~Tb}_{x} \mathrm{Co}_{4} \mathrm{O}_{9} \mathrm{~K}_{y}(x=0, y=0$; $x=0.05, y=0 ; x=0.5, y=0 ; x=0.05, y=0.1 ; x=0.5, y=0.1$ ). (a) $\rho-T$, (b) $S-T$, and (c) $S^{2} / \rho-T$. 

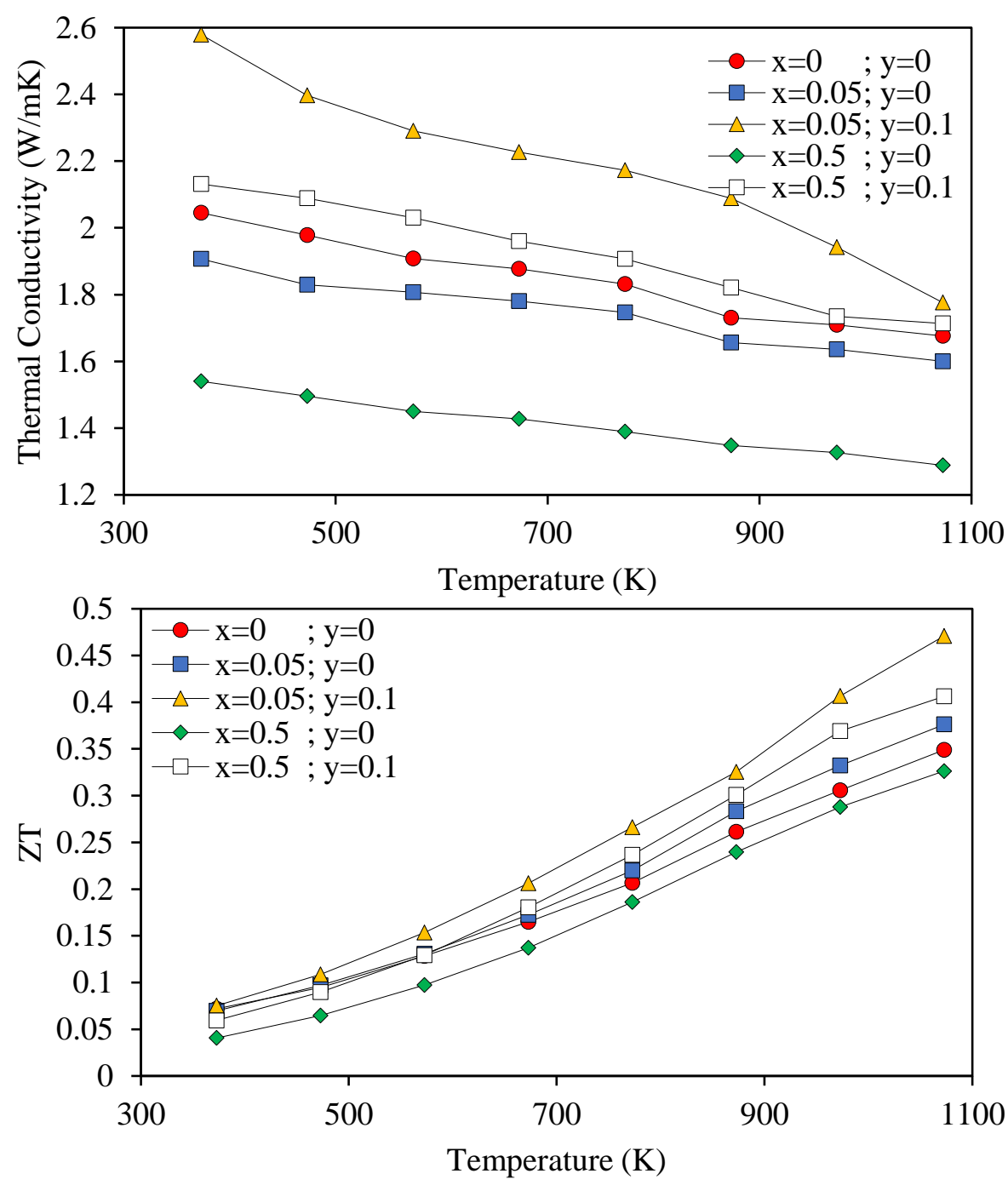

Figure 8-2: Temperature dependence of (a) thermal conductivity ( $\kappa-T)$, and (b) thermoelectric figure of merit (ZT) for $\mathrm{Ca}_{3-x} \mathrm{~Tb}_{x} \mathrm{Co}_{4} \mathrm{O}_{9} \mathrm{~K}_{y}(x=0, y=0 ; \mathrm{x}=0.05, \mathrm{y}=0 ; \mathrm{x}=0.5, \mathrm{y}=0 ; x=0.05, y=0.1$;

$$
x=0.5, y=0.1 \text { ). }
$$

Figure 8-2 shows the variation of the $\kappa$ and $Z T$ for the pellets with the different concentrations levels as a function of temperature for the $\mathrm{Ca}_{3-\mathrm{x}} \mathrm{Tb}_{\mathrm{x}} \mathrm{Co}_{4} \mathrm{O}_{9} \mathrm{~K}_{y}$ samples. As discussed previously, the influence of substituting calcium by $\mathrm{Tb}$ greatly decreases the $\kappa$ of the $\mathrm{Ca}_{3} \mathrm{Co}_{4} \mathrm{O}_{9}$ ceramics with the increasing doping level of $\mathrm{Tb}$. In contract, further addition of $\mathrm{K}^{+}$as second dopant greatly increases the $\kappa$ values above those of the pristine $\mathrm{Ca}_{3} \mathrm{Co}_{4} \mathrm{O}_{9}$. Therefore, the negative influence of $\mathrm{K}^{+}$doping on the thermal transport properties of the $\mathrm{Ca}_{3} \mathrm{Co}_{4} \mathrm{O}_{9}$ is confirmed. 
Figure 8-2 (b) shows the improved figure of merit $Z T$ for the different doping levels of $\mathrm{Tb}$ and $\mathrm{K}^{+}$. Previously reported, doping $\mathrm{Tb}$ into $\mathrm{Ca}_{3} \mathrm{Co}_{4} \mathrm{O}_{9}$ achieves a $Z T \sim 0.37$ at $1073 \mathrm{~K}$ which provides a greater starting point than the pristine $\mathrm{Ca}_{3} \mathrm{Co}_{4} \mathrm{O}_{9}$ value of $Z T \sim 0.35$ at $1073 \mathrm{~K}$. There is a supplementary increase of $Z T$ with the further inclusion of $\mathrm{K}^{+}$at a doping level $\mathrm{y}=0.10$, reaching a high $Z T$ value of 0.47 at $1073 \mathrm{~K}$ for the $\mathrm{Ca}_{2.95} \mathrm{~Tb}_{0.05} \mathrm{Co}_{4} \mathrm{O}_{9} \mathrm{~K}_{0.1}$ samples. Particularly, this $Z T$ value is greater than the previously reported for $\mathrm{Ca}_{3} \mathrm{Co}_{4} \mathrm{O}_{9} \mathrm{~K}_{0.1}$, such improvement is primarily due to the inclusion of $\mathrm{Tb}$ in this dual doping material. The obtained $Z T$ value represents roughly a $\sim 35 \%$ improvement in comparison to that of the $\mathrm{Ca}_{3} \mathrm{Co}_{4} \mathrm{O}_{9}$ baseline.

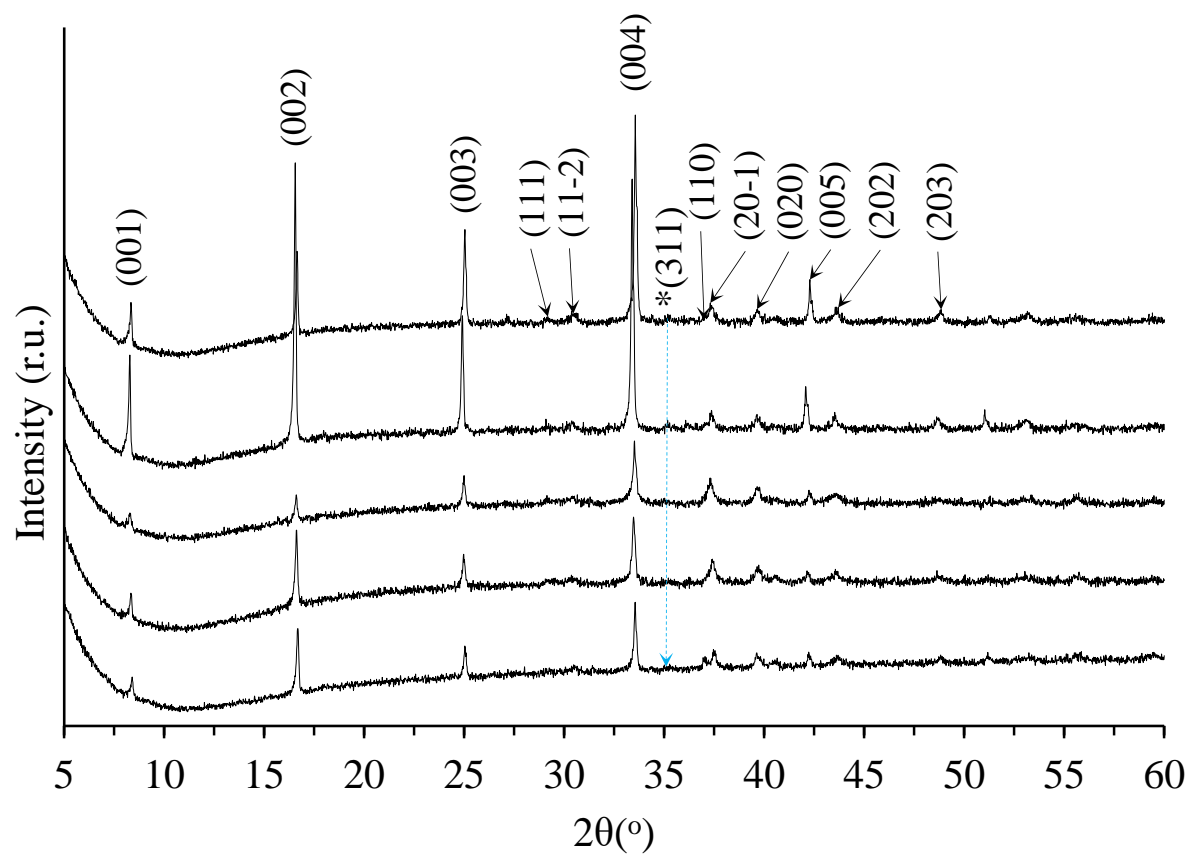

Figure 8-3: XRD powder diffraction patterns for the undoped $\mathrm{Ca}_{3} \mathrm{Co}_{4} \mathrm{O}_{9}$ and the doped $\mathrm{Ca}_{3-x} \mathrm{~Tb}_{x} \mathrm{Co}_{4} \mathrm{O}_{9} \mathrm{~K}_{y}(\mathrm{x}=0.05, \mathrm{y}=0 ; \mathrm{x}=0.5, \mathrm{y}=0 ; x=0.05, y=0.1 ; x=0.5, y=0.1)$ samples. In all samples, monoclinic $\mathrm{Ca}_{3} \mathrm{Co}_{4} \mathrm{O}_{9}$ were observed (ICDD cards \#4-16-860). Nonetheless, a minor amount of $\mathrm{Co}_{3} \mathrm{O}_{4}$ was observed as indicated by the marked peaks (ICDD cards \# 4-8-3173). 
The XRD powder diffraction patterns for the undoped $\mathrm{Ca}_{3} \mathrm{Co}_{4} \mathrm{O}_{9}$ and the doped $\mathrm{Ca}_{3}$ ${ }_{x} \mathrm{~Tb}_{x} \mathrm{Co}_{4} \mathrm{O}_{9} \mathrm{~K}_{y}$ obtained at room temperature conditions from ground powders of the sintered pellets are shown in Figure 8-3. For visualization purposes, patterns have been plotted with a small offset. XRD characteristic peaks obtained from the samples can be indexed as those from $\mathrm{Ca}_{3} \mathrm{Co}_{4} \mathrm{O}_{9}$ phase with monoclinic symmetry. Nonetheless, there is a very small presence of characteristic diffraction peaks from a minor amount of $\mathrm{Co}_{3} \mathrm{O}_{4}$ as indicated by the marked peaks, such $\mathrm{Co}_{3} \mathrm{O}_{4}$ secondary phases are present in very small amounts in all samples including the pristine $\mathrm{Ca}_{3} \mathrm{Co}_{4} \mathrm{O}_{9}$. Additionally, the patterns exhibit a small shift to a higher Bragg's angle of the peaks for those concentrations with $\mathrm{Tb}$, as the doping level of $\mathrm{Tb}$ increases the Bragg's angle shift to a higher degree. The shift to a higher Bragg's angle indicates Tb has been incorporated to the crystal and increased the lattice parameter values for $\mathrm{Ca}_{3} \mathrm{Co}_{4} \mathrm{O}_{9}$ as shown in previous chapters. On the other hand, there is not significant shift of the XRD peaks with the incorporation of $\mathrm{K}^{+}$as a second dopant in the dual doped material.

Table 8-2: Lattice parameters $a, b_{1}, b_{2}, c$, and $\beta$ for $\mathrm{Ca}_{3-x} \mathrm{~Tb}_{x} \mathrm{Co}_{4} \mathrm{O}_{9} K_{y}(x=0, y=0 ; x=0.05, y=0$; $x=0.5, y=0 ; x=0.05, y=0.1 ; x=0.5, y=0.1)$ samples. $b_{1}$ and $b_{2}$ refer to $b$-axis lattice parameters in the rock-salt layer $\mathrm{Ca}_{2} \mathrm{CoO}_{3}$ and the $\mathrm{CoO}_{2}$ layer, respectively.

\begin{tabular}{llllll}
\hline $\mathrm{Ca}_{3-\mathrm{x}} \mathrm{Tb}_{\mathrm{x}} \mathrm{Co}_{4} \mathrm{O}_{9} \mathrm{~K}_{\mathrm{y}}$ & $a(\AA)$ & $b_{1}(\AA)$ & $b_{2}(\AA)$ & $c(\AA)$ & $\beta\left(^{\circ}\right)$ \\
\hline$x=0 \quad ; y=0$ & 4.874 & 4.550 & 2.786 & 10.819 & 98.885 \\
$x=0.05 ; y=0$ & 4.847 & 4.546 & 2.785 & 10.832 & 98.432 \\
$x=0.5 ; y=0$ & 4.842 & 4.542 & 2.772 & 10.804 & 98.357 \\
$x=0.05 ; y=0.1$ & 4.848 & 4.546 & 2.786 & 10.849 & 98.482 \\
$x=0.5 ; y=0.1$ & 4.836 & 4.542 & 2.772 & 10.792 & 98.136 \\
\hline
\end{tabular}


The calculated lattice parameter values for samples with the different doping level are listed in Table 8-2. After calculating the lattice parameter values it is observed that with the increasing $\mathrm{Tb}$ doping level, there is a decrease in the lattice parameter values $a, b_{1}, b_{2}, c$, and $\beta$, with the only exception of a small increase in the lattice parameter for the lower doping level of $\mathrm{Tb}$. Compared to the effect of the stoichiometric substitution of the $\mathrm{Tb}$, the non-stoichiometric addition of $\mathrm{K}^{+}$does not show an important impact on the lattice parameter values in the dual doping materials, suggesting that $\mathrm{K}^{+}$is not present in the crystal lattice.
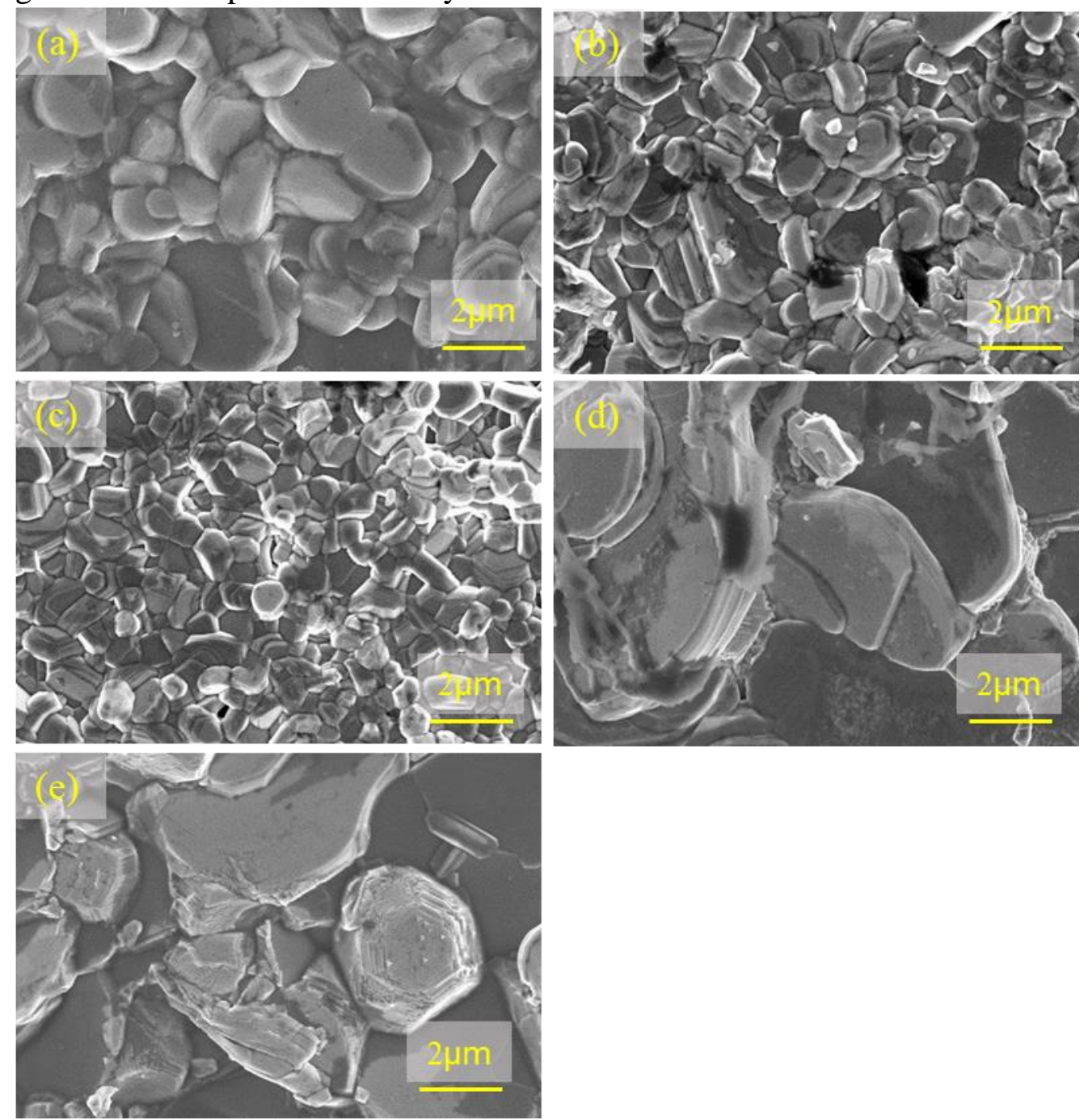

Figure 8-4: SEM images from the top surface of $\mathrm{Ca}_{3-x} \mathrm{~Tb}_{x} \mathrm{Co}_{4} \mathrm{O}_{9} K_{y}(x=0, y=0 ; x=0.05, y=0$; $x=0.5, y=0 ; x=0.05, y=0.1 ; x=0.5, y=0.1$ ) samples after sintering stage. (a) $\mathrm{Ca}_{3} \mathrm{Co}_{4} \mathrm{O}_{9}$, (b) $\mathrm{Ca}_{2.95} \mathrm{~Tb}_{0.05} \mathrm{Co}_{4} \mathrm{O}_{9}$, (c) $\mathrm{Ca}_{2.5} \mathrm{~Tb}_{0.5} \mathrm{Co}_{4} \mathrm{O}_{9}$ (d) $\mathrm{Ca}_{2.95} \mathrm{~Tb}_{0.05} \mathrm{Co}_{4} \mathrm{O}_{9} \mathrm{~K}_{0.1}$, (e) $\mathrm{Ca}_{2.5} \mathrm{~Tb}_{0.5} \mathrm{Co}_{4} \mathrm{O}_{9} \mathrm{~K}_{0.1}$. 
Figure 8-4 shows the top-view SEM images taken from the pressed plane of the $\mathrm{Ca}_{3}$ ${ }_{x} \mathrm{~Tb}_{x} \mathrm{Co}_{4} \mathrm{O}_{9} \mathrm{~K}_{y}$ pellets after sintering. As discussed previously, Tb decreases considerably the grain size of the calcium cobaltite and there is not improvement on the crystal texture nor grain alignment with such dopant. In contrast, the incorporation of $\mathrm{K}^{+}$as a second dopant in the dual doped material has a positive influence on the development of the crystal texture and the grain growth. The micron-sized crystals exhibit a plate-shape morphology.
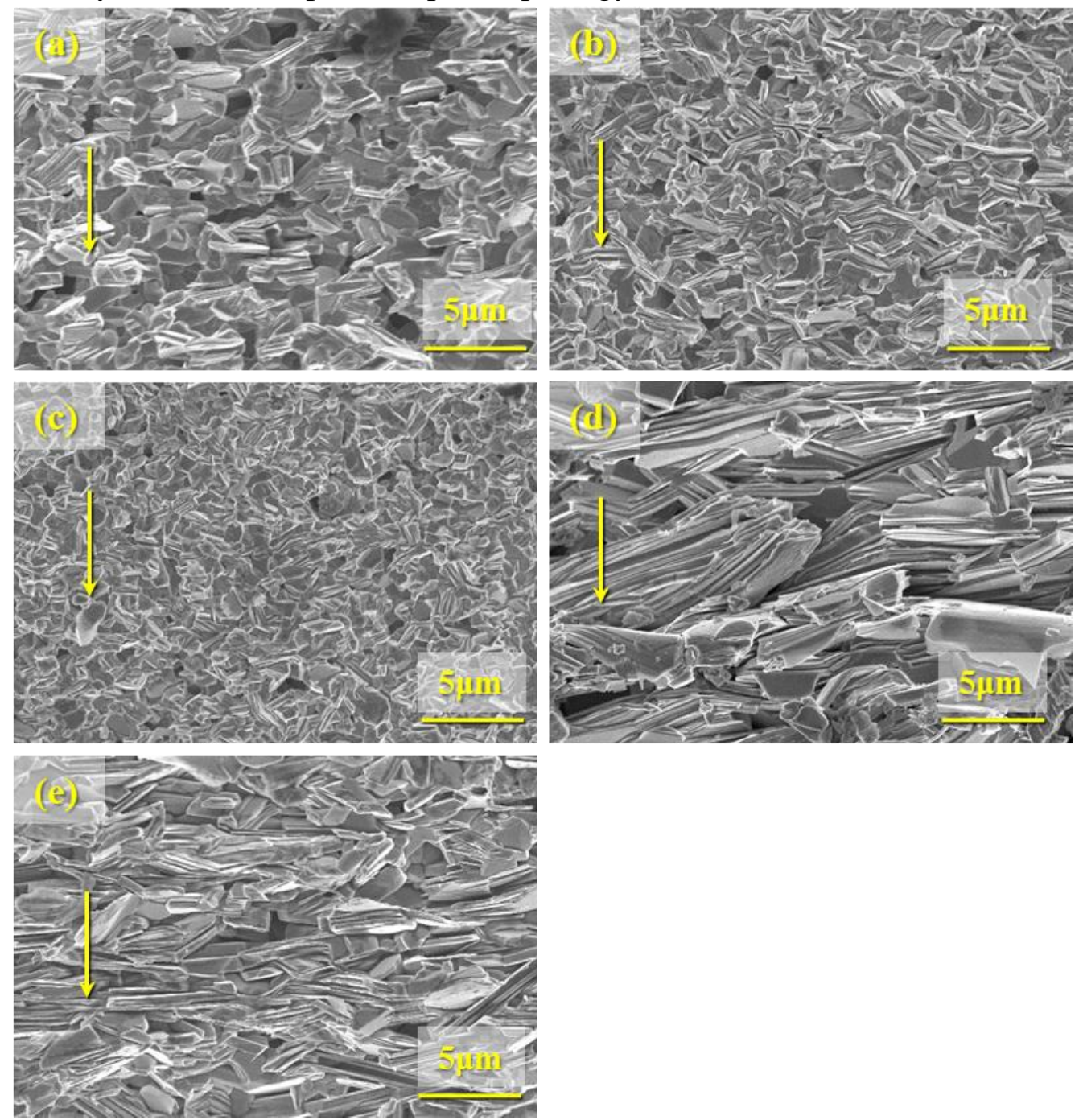

Figure 8-5: Cross-sectional SEM images from the fractured surface of $\mathrm{Ca}_{3-x} \mathrm{~Tb}_{x} \mathrm{Co}_{4} \mathrm{O}_{9} \mathrm{~K}_{y}(x=0$, $y=0 ; x=0.05, y=0 ; x=0.5, y=0 ; x=0.05, y=0.1 ; x=0.5, y=0.1)$ samples after sintering stage.

(a) $\mathrm{Ca}_{3} \mathrm{Co}_{4} \mathrm{O}_{9}$, (b) $\mathrm{Ca}_{2.95} \mathrm{~Tb}_{0.05} \mathrm{Co}_{4} \mathrm{O}_{9}$, (c) $\mathrm{Ca}_{2.5} \mathrm{~Tb}_{0.5} \mathrm{Co}_{4} \mathrm{O}_{9}$, (d) $\mathrm{Ca}_{2.95} \mathrm{~Tb}_{0.05} \mathrm{Co}_{4} \mathrm{O}_{9} \mathrm{~K}_{0.1}$, (e) $\mathrm{Ca}_{2.5} \mathrm{~Tb}_{0.5} \mathrm{Co}_{4} \mathrm{O}_{9} \mathrm{~K}_{0.1}$. 
Figure 8-5 shows the cross-sectional SEM images taken from the fractured surface of the $\mathrm{Ca}_{3}$ ${ }_{x} \mathrm{~Tb}_{x} \mathrm{Co}_{4} \mathrm{O}_{9} \mathrm{~K}_{y}$ pellets after sintering. The arrow in each picture indicates the pressing direction of the pellets. The incorporation of $\mathrm{K}^{+}$produces a gradual increase of the size of the crystals parallel to the $a-b$ plane of the elongated crystal grains, while remained with a similar size in the $c$-axes. The SEM images clearly show samples with large anisotropy with crystals having the larger dimension in direction to the $a-b$ plane of the monoclinic $\mathrm{Ca}_{3} \mathrm{Co}_{4} \mathrm{O}_{9}$ and the $c$-axis parallel to the pressed direction of the pellets. Dual doped samples exhibit a better texture and preferentially aligned crystal orientation.

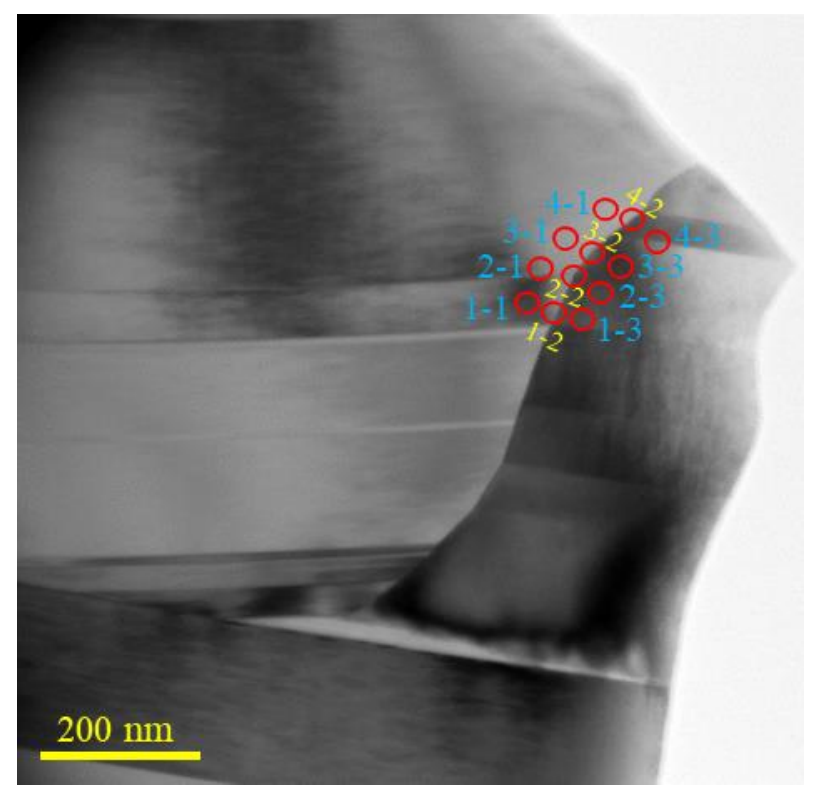

Figure 8-6: TEM images showing the nanostructure of the $\mathrm{Ca}_{2.95} \mathrm{~Tb}_{0.05} \mathrm{Co}_{4} \mathrm{O}_{9} \mathrm{~K}_{0.1}$ sample. (a) The grain boundary from the crystals with different c-axis orientations. The red circles on the TEM images indicate the electron beam sample areas for the EDS data acquisition

The examination of $\mathrm{Tb}$ doping into $\mathrm{Ca}_{3} \mathrm{Co}_{4} \mathrm{O}_{9}$ and his crystal structure was previously examined by TEM. In Figure 8-6, the TEM examination performed on the $\mathrm{Ca}_{2.95} \mathrm{~Tb}_{0.05} \mathrm{Co}_{4} \mathrm{O}_{9} \mathrm{~K}_{0.1}$ sample having the best crystal texture and performance is shown. The examination shows the nanolamella structure from three adjacent grains with different $c$-axes, where the arrows shown 
are in parallel direction to the $c$-axes. The adjacent crystals have a large amount of low grain boundary angle between them. Furthermore, no secondary phase was present in the TEM results, meaning those secondary phases exhibited by XRD results could exist in only very small local areas. Additionally, there is not presence of secondary phases at the GBs. EDS was used to further determine the atomic ratio of the different elements and the chemistry on the GBs and crystal lattice of the dual-doped $\mathrm{Ca}_{3} \mathrm{Co}_{4} \mathrm{O}_{9}$. The spots in Figure 8-6 correspond to the location of EDS between the adjacent grains.

Table 8-3: Chemistry of the grain interior and grain boundaries of $\mathrm{Ca}_{2.95} \mathrm{~Tb}_{0.05} \mathrm{Co}_{4} \mathrm{O}_{9} \mathrm{~K}_{0.1}$ for different sites labeled in the TEM image in Figure 8-6.

\begin{tabular}{llllllllll}
\hline $\mathrm{At} \%$ & $1-1$ & $1-2$ & $1-3$ & & $\mathrm{At} \%$ & $3-1$ & $3-2$ & $3-3$ \\
\hline $\mathrm{O}$ & 67.99 & 69.85 & 69.47 & & $\mathrm{O}$ & 70.56 & 70.80 & 69.42 \\
$\mathrm{Ca}$ & 14.34 & 13.36 & 11.39 & & $\mathrm{Ca}$ & 13.11 & 12.00 & 11.99 \\
$\mathrm{Co}$ & 17.34 & 16.48 & 18.94 & & $\mathrm{Co}$ & 15.97 & 16.74 & 18.31 \\
$\mathrm{~Tb}$ & 0.33 & 0.23 & 0.2 & & $\mathrm{~Tb}$ & 0.36 & 0.41 & 0.29 \\
$\mathrm{~K}$ & - & 0.08 & - & & $\mathrm{K}$ & - & 0.04 & - \\
\hline $\mathrm{At} \%$ & $2-1$ & $2-2$ & $2-3$ & & $\mathrm{At} \%$ & $4-1$ & $4-2$ & $4-3$ \\
\hline $\mathrm{O}$ & 67.22 & 68.42 & 69.17 & & $\mathrm{O}$ & 69.70 & 71.17 & 69.25 \\
$\mathrm{Ca}$ & 14.47 & 12.60 & 11.79 & & $\mathrm{Ca}$ & 13.61 & 13.08 & 14.32 \\
$\mathrm{Co}$ & 17.90 & 18.72 & 18.62 & & $\mathrm{Co}$ & 16.43 & 15.35 & 16.08 \\
$\mathrm{~Tb}$ & 0.42 & 0.21 & 0.41 & & $\mathrm{~Tb}$ & 0.26 & 0.36 & 0.35 \\
$\mathrm{~K}$ & - & 0.05 & - & & $\mathrm{K}$ & - & 0.04 & - \\
\hline
\end{tabular}

The atomic ratio for each EDS spot is summarized in Table 8-3. In previous samples doped with $\mathrm{Tb}$ only, the EDS examination showed the depletion of $\mathrm{Tb}$ at the grain boundaries while also $\mathrm{Tb}$ present in the lattice. For the dual doped materials, local areas were $\mathrm{Tb}$ is depleted at the grain boundaries must exist as reported previously. Nonetheless, EDS results obtained are inconclusive and depletion in the dual doped material could not be confirmed. Results confirmed the presence of non-stoichiometric addition $\mathrm{K}^{+}$segregated at the grain boundaries in the polycrystalline $\mathrm{Ca}_{3-x} \mathrm{~Tb}_{x} \mathrm{Co}_{4} \mathrm{O}_{9} \mathrm{~K}_{y}$. 


\section{5 Discussion}

The above results clearly demonstrated that the dual-dopant $\mathrm{Tb}$ and $\mathrm{K}^{+}$affected both the crystal texture and the electrical performance of the $\mathrm{Ca}_{3-x} \mathrm{~Tb}_{x} \mathrm{Co}_{4} \mathrm{O}_{9} \mathrm{~K}_{y}$. The crystal texture development along the $a-b$ plane and the enhanced orientation of grains greatly improve the electrical transport properties in the polycrystalline $\mathrm{Ca}_{3} \mathrm{Co}_{4} \mathrm{O}_{9}$. The crystal structure of $\mathrm{Ca}_{3} \mathrm{Co}_{4} \mathrm{O}_{9}$ is comprised of two misfit layered subsystems aligned in the $c$-axis, the $\mathrm{CoO}_{2}$ layer being electrically conductive and the $\mathrm{CaCo}_{2} \mathrm{O}_{3}$ being electrically insulating. As a result of this intrinsic anisotropic characteristic, the resistivity in the $a-b$ basal plane of the monoclinic unit cell is significantly lower than along the $c$-axis. ${ }^{[9]}$ As shown in the SEM image for $\mathrm{Ca}_{3-x} \mathrm{~Tb}_{x} \mathrm{Co}_{4} \mathrm{O}_{9}$ samples and explained previously, $\mathrm{Tb}$ does not promote crystal texture or a better grain orientation which leads to a deteriorated electrical resistivity. Nonetheless, the oversized $\mathrm{K}^{+}$segregated at the grain boundaries decreases the grain boundary energy and facilitates the fast diffusion along the grain boundaries and the crystal texture development ${ }^{[27]}$. The SEM images for the dual doped $\mathrm{Ca}_{3-x} \mathrm{~Tb}_{x} \mathrm{Co}_{4} \mathrm{O}_{9} \mathrm{~K}_{y}$ shows that with the appropriate doping level the desired crystal texture and orientation is achieved. Most remarkably, even at the higher doping level of $\mathrm{Tb} x=0.5$ the further addition of $\mathrm{K}^{+}$promoted a better crystal texture and orientation. It has been reported that these microstructure improvements

contribute to an enhanced carrier mobility ${ }^{[32,33]}$. Additionally, the electrical conductivity $(\sigma)$ could be expressed as $\sigma=n e \mu$, where $\mu$ is the carrier mobility, $e$ is the elementary positive charge constant, and $n$ is the number density of electrons. Thus, the reduction of $\rho$ in the dual doped $\mathrm{Ca}_{3-x} \mathrm{~Tb}_{x} \mathrm{Co}_{4} \mathrm{O}_{9} \mathrm{~K}_{y}$ samples is a direct result from an improved carrier mobility from the crystal texture development.

Previously, the improvement in the $\mathrm{S}$ due to $\mathrm{Tb}$ substation was discussed and attributed to a decrease in the carrier concentration of the $\mathrm{Ca}_{3} \mathrm{Co}_{4} \mathrm{O}_{9}$ ceramics. It has been reported that the 
magnitude of $S$ could be increased by increasing the concentration fraction of $\mathrm{Co}^{4+}$ in comparison to the $\mathrm{Co}^{3+}$ in the $\mathrm{CoO}_{2}$ layer. Therefore, the increase of the Seebeck of the Tb doped samples is attributed to the decrease in the carrier concentration. On the other hand, the further enhancement in the $S$ due to $\mathrm{K}^{+}$non-stoichiometric addition in the dual doped $\mathrm{Ca}_{3-x} \mathrm{~Tb}_{x} \mathrm{Co}_{4} \mathrm{O}_{9} \mathrm{~K}_{y}$ ceramics is potentially accredited to the grain boundary segregation of $\mathrm{K}^{+}$as is the case in the $\mathrm{Ca}_{3} \mathrm{Co}_{4} \mathrm{O}_{9} \mathrm{~K}_{y}$ samples. ${ }^{[27]}$ Dopant segregation at the grain boundaries acts as a block layer in the bulk material originated by a band offset among the grain boundary and the grain interior. ${ }^{[19]}$ Thus, the further increase of $S$ in the dual doped material could be associated to the selective filtering of low energy carriers at the grain boundaries due to the reduced carrier concentration ${ }^{[16,17]}$.

This study further confirms that the segregation of $\mathrm{K}^{+}$is primarily attributed to the large difference in its ionic radius in comparison with the $\mathrm{Ca}_{3} \mathrm{Co}_{4} \mathrm{O}_{9}$ lattice sites and no further interaction with $\mathrm{Tb}$ displaced $\mathrm{K}^{+}$from the grain boundaries. However, for this system with dual dopants, $\mathrm{Tb}$ added to the ceramics simultaneously did not depleted at all the analyzed grain boundaries as in the case of $\mathrm{Ca}_{3-x} \mathrm{~Tb}_{x} \mathrm{Co}_{4} \mathrm{O}_{9}$. Yet, in multiple areas the $\mathrm{Tb}$ is depleted due to its smaller ionic radii. Such complex interactions provide an interesting platform to keep studying dual dopant system with different dopants.

Overall, the enriched electronic properties of the dual doped material achieved an enhanced electrical PF value of $0.77 \mathrm{mWm}^{-1} \mathrm{~K}^{-2}$ at $1073 \mathrm{~K}$. However, it was further confirmed that the overall $\kappa$ is not improved for the doped samples in comparison to the undoped $\mathrm{Ca}_{3} \mathrm{Co}_{4} \mathrm{O}_{9}$ due to the inclusion of the light atom $\mathrm{K}^{+}$. Still, the overall $Z T$ of the best concentration of dual stoichiometric substitution of $\mathrm{Tb}$ and non-stoichiometric addition of $\mathrm{K}^{+}$enhanced the TE performance to a value of 0.47 at $1073 \mathrm{~K}$, which represents a $35 \%$ increase with the pristine $\mathrm{Ca}_{3} \mathrm{Co}_{4} \mathrm{O}_{9}$. Notwithstanding, 
$\mathrm{Tb}$ remains a viable candidate to promote phonon scattering events and to further boost the Seebeck coefficient in dual doped materials.

\section{6 Conclusion}

This work shows that non-stoichiometric addition of oversized dopants in a dual doping approach segregate to the grain boundary and drastically improve the $S$ and simultaneously reducing the $\rho$ due to the developed crystal texture. Both values showed a better performance than the baseline counterpart, with a $28 \%$ reduction in $\rho$ and a $10 \%$ increase in the $S$. This further confirm the importance and versatility of the selection of dopants and show how the GB control in polycrystalline $\mathrm{Ca}_{3} \mathrm{Co}_{4} \mathrm{O}_{9}$ have a big impact on electrical transport properties. $\mathrm{Tb}$ and $\mathrm{K}^{+}$doping resulted in microstructure evolution, improved crystal grain alignment and segregation and depletion of dopants at the GB. The crystal texture development on $\mathrm{Ca}_{3-x} \mathrm{~Tb}_{x} \mathrm{Co}_{4} \mathrm{O}_{9} \mathrm{~K}_{y}$ materials facilitate the charge carrier mobility which leaded to a improved $\rho$ with a value of $47.7 \mu \Omega \mathrm{m}$ at $1073 \mathrm{~K}$. Whereas GB segregation of $\mathrm{K}^{+}$doping works as a block layer to decrease the $n$ and simultaneously increase the $S$ to a value of $210 \mu \mathrm{VK}^{-1}$. The combined interactions of $\mathrm{Tb}$ and $\mathrm{K}^{+}$ doping greatly enhanced the electrical PF a 40\%, the thermal conductivity, on the other hand, increased an unfavorable $6 \%$ for concentrations with the best electrical PF of $0.77 \mathrm{mWm}^{-1} \mathrm{~K}^{-2}$ due to the nature and level of the dopants. Nonetheless, the overall ZT was improved by the best concentration $\mathrm{Ca}_{2.95} \mathrm{~Tb}_{0.05} \mathrm{Co}_{4} \mathrm{O}_{9} \mathrm{~K}_{0.1}$ to a value of 0.47 at $1073 \mathrm{~K}$ which represents $\sim 35 \%$ increase to the value of the pristine $\mathrm{Ca}_{3} \mathrm{Co}_{4} \mathrm{O}_{9}$. The outcome of this work shows the potential of grain boundary engineering while also conveying the imperative need for more research on the use of multiple dopants to further understand the largely unexplored complexity of the grain boundary effects in polycrystalline $\mathrm{Ca}_{3} \mathrm{Co}_{4} \mathrm{O}_{9}$. 


\section{References}

1. Forman, C., et al., Estimating the global waste heat potential. Renewable and Sustainable Energy Reviews, 2016. 57: p. 1568-1579.

2. Snyder, G.J. and E.S. Toberer, Complex thermoelectric materials. Nature Materials, 2008. 7: p. 105.

3. Fu, C., et al., Realizing high figure of merit in heavy-band p-type half-Heusler thermoelectric materials. Nature Communications, 2015. 6: p. 8144.

4. Champier, D. Thermoelectric Generators: A Review of Present and Future Applications. in 3rd International Congress on Energy Efficiency and Energy Related Materials (ENEFM2015). 2017. Cham: Springer International Publishing.

5. Zhao, L.-D., et al., Ultralow thermal conductivity and high thermoelectric figure of merit in SnSe crystals. Nature, 2014. 508: p. 373.

6. Poudel, B., et al., High-Thermoelectric Performance of Nanostructured Bismuth Antimony Telluride Bulk Alloys. Science, 2008. 320(5876): p. 634.

7. Joshi, G., et al., Enhanced Thermoelectric Figure-of-Merit in Nanostructured p-type Silicon Germanium Bulk Alloys. Nano Letters, 2008. 8(12): p. 4670-4674.

8. Feng, Y., et al., Metal oxides for thermoelectric power generation and beyond. Advanced Composites and Hybrid Materials, 2018. 1(1): p. 114-126.

9. Shikano, M. and R. Funahashi, Electrical and thermal properties of single-crystalline $\left(\mathrm{Ca}_{2} \mathrm{CoO}_{3}\right)_{0.7} \mathrm{CoO}_{2}$ with a $\mathrm{Ca}_{3} \mathrm{Co}_{4} \mathrm{O}_{9}$ structure. Applied Physics Letters, 2003. 82(12): p. 1851-1853.

10. Fergus, J.W., Oxide materials for high temperature thermoelectric energy conversion. Journal of the European Ceramic Society, 2012. 32(3): p. 525-540.

11. Zhang, F.P., et al., Effects of Pr doping on thermoelectric transport properties of $\mathrm{Ca}_{3-x} \mathrm{Pr}_{x} \mathrm{CO}_{4} \mathrm{O}_{9}$. Solid State Sciences, 2011. 13(7): p. 1443-1447.

12. Nong, N.V., C.-J. Liu, and M. Ohtaki, High-temperature thermoelectric properties of late rare earth-doped $\mathrm{Ca}_{3} \mathrm{Co}_{4} \mathrm{O}_{9+\delta}$. Journal of Alloys and Compounds, 2011. 509(3): p. 977981.

13. Klyndyuk, A.I. and I.V. Matsukevich, Synthesis and properties of $\mathrm{Ca}_{2.8} \mathrm{Ln}_{0.2} \mathrm{Co}_{4} \mathrm{O}_{9+\delta}(\mathrm{Ln}$ $=\mathrm{La}, \mathrm{Nd}$, Sm, Tb-Er) solid solutions. Inorganic Materials, 2012. 48(10): p. 1052-1057.

14. Saini, S., et al., Terbium Ion Doping in $\mathrm{Ca}_{3} \mathrm{Co}_{4} \mathrm{O}_{9}$ : A Step towards High-Performance Thermoelectric Materials. Scientific Reports, 2017. 7: p. 44621.

15. Muthiah, S., et al., Conducting grain boundaries enhancing thermoelectric performance in doped $\mathrm{Mg}_{2} \mathrm{Si}$. Applied Physics Letters, 2013. 103(5): p. 053901.

16. Meng, X., et al., Grain Boundary Engineering for Achieving High Thermoelectric Performance in n-Type Skutterudites. Advanced Energy Materials, 2017. 7(13): p. 1602582.

17. Hossein-Babaei, F., S. Masoumi, and A. Noori, Linking thermoelectric generation in polycrystalline semiconductors to grain boundary effects sets a platform for novel Seebeck effect-based sensors. Journal of Materials Chemistry A, 2018. 6(22): p. 10370-10378.

18. Zong, P.-a., et al., Skutterudite with graphene-modified grain-boundary complexion enhances zT enabling high-efficiency thermoelectric device. Energy \& Environmental Science, 2017. 10(1): p. 183-191. 
19. Mehdizadeh Dehkordi, A., et al., Large Thermoelectric Power Factor in Pr-Doped $\mathrm{SrTiO}_{3-\delta}$ Ceramics via Grain-Boundary-Induced Mobility Enhancement. Chemistry of Materials, 2014. 26(7): p. 2478-2485.

20. Zhang, Q., et al., Realizing high-performance thermoelectric power generation through grain boundary engineering of skutterudite-based nanocomposites. Nano Energy, 2017. 41: p. 501-510.

21. Mun, H., et al., Boundary Engineering for the Thermoelectric Performance of Bulk Alloys Based on Bismuth Telluride. ChemSusChem, 2015. 8(14): p. 2312-2326.

22. Mao, J., et al., Manipulation of ionized impurity scattering for achieving high thermoelectric performance in n-type $\mathrm{Mg}_{3} \mathrm{Sb}_{2}$-based materials. Proceedings of the National Academy of Sciences of the United States of America, 2017. 114(40): p. 10548-10553.

23. Kim, S.I., et al., Dense dislocation arrays embedded in grain boundaries for highperformance bulk thermoelectrics. Science, 2015. 348(6230): p. 109.

24. Boyle, C., et al., Grain boundary segregation and thermoelectric performance enhancement of bismuth doped calcium cobaltite. Journal of the European Ceramic Society, 2016. 36(3): p. 601-607.

25. Boyle, C., et al., Competing dopants grain boundary segregation and resultant seebeck coefficient and power factor enhancement of thermoelectric calcium cobaltite ceramics. Ceramics International, 2017. 43(14): p. 11523-11528.

26. Carvillo, P., et al., Thermoelectric Performance Enhancement of Calcium Cobaltite through Barium Grain Boundary Segregation. Inorganic Chemistry, 2015. 54(18): p. 90279032.

27. Romo-De-La-Cruz, C., et al., Role of oversized dopant potassium on the nanostructure and thermoelectric performance of calcium cobaltite ceramics. Sustainable Energy \& Fuels, 2018. 2(4): p. 876-881.

28. Boyle, C., et al., Improving the thermoelectric performance and thermal stability of $\mathrm{Ca}_{3} \mathrm{Co}_{4} \mathrm{O}_{9}$ ceramics by sintering in oxygen atmosphere. Journal of Sol-Gel Science and Technology, 2018. 85(3): p. 712-722.

29. Sotelo, A., et al., Improvement of thermoelectric properties of $\mathrm{Ca}_{3} \mathrm{Co}_{4} \mathrm{O}_{9}$ using soft chemistry synthetic methods. Journal of the European Ceramic Society, 2012. 32(10): p. 2415-2422.

30. Chen, S., et al., Effect of precursor calcination temperature on the microstructure and thermoelectric properties of $\mathrm{Ca}_{3} \mathrm{Co}_{4} \mathrm{O}_{9}$ ceramics. Journal of Sol-Gel Science and Technology, 2012. 64(3): p. 627-636.

31. Delorme, F., et al., Thermoelectric properties of $\mathrm{Ca}_{3} \mathrm{Co}_{4} \mathrm{O}_{9}-\mathrm{Co}_{3} \mathrm{O}_{4}$ composites. Ceramics International, 2015. 41(8): p. 10038-10043.

32. Mikami, M., et al., Enhancement of Electrical Properties of the Thermoelectric Compound $\mathrm{Ca}_{3} \mathrm{Co}_{4} \mathrm{O}_{9}$ through Use of Large-grained Powder. Journal of Materials Research, 2011. 20(9): p. 2491-2497.

33. Guilmeau, E., et al., Thermoelectric properties-texture relationship in highly oriented $\mathrm{Ca}_{3} \mathrm{Co}_{4} \mathrm{O}_{9}$ composites. Applied Physics Letters, 2004. 85(9): p. 1490-1492. 


\section{Chapter 9. Systematic Dual Doping Influence on the Thermoelectric Performance of $\mathrm{Ca}_{3} \mathrm{Co}_{4} \mathrm{O}_{9}$}

\section{1 Achieving a high-record ZT performance}

P-type oxide calcium cobaltite $\mathrm{Ca}_{3} \mathrm{Co}_{4} \mathrm{O}_{9-\delta}$ is an emerging promising candidate for thermoelectric (TE) applications and possesses high thermal stability in air at high temperatures, low cost, light weight, and non-toxicity. Single crystal $\mathrm{Ca}_{3} \mathrm{Co}_{4} \mathrm{O}_{9-\delta}$ exhibits excellent $\mathrm{TE}$ performance, approaching that of the well-developed conventional TE materials. However, TE energy conversion efficiency of polycrystalline $\mathrm{Ca}_{3} \mathrm{Co}_{4} \mathrm{O}_{9-\delta}$ ceramics is currently only about $\sim 30$ $60 \%$ of that of the single crystals. A methodical dual doping approach to improve the thermoelectric performance of $\mathrm{Ca}_{3} \mathrm{Co}_{4} \mathrm{O}_{9-\delta}$ ceramics is presented. Different from the conventional cation substitution approach, terbium and bismuth were introduced through both the cation stoichiometric substitution and novel non-stoichiometric addition, respectively. Those dopants subsequently display the depletion and segregation at the grain boundaries, which affect the crystal texture, grain boundary density, and dramatically impacted the TE energy conversion efficiency of the polycrystalline ceramic oxide. The outstanding ZT $\sim 0.89$ at $1073 \mathrm{~K}$ obtained for the highperformance bulk scale oxide ceramics with a stoichiometry chemistry $\mathrm{Ca}_{2.95} \mathrm{~Tb}_{0.05} \mathrm{Co}_{4} \mathrm{O}_{9} \mathrm{Bi}_{0.25}$ using the dual doping approach outperform the $\mathrm{Ca}_{3} \mathrm{Co}_{4} \mathrm{O}_{9-\delta}$ single crystals and outperform the state-of-the-art $\mathrm{SiGe}$. Most of all, $\mathrm{Ca}_{3} \mathrm{Co}_{4} \mathrm{O}_{9-\delta}$ ceramics are having just 5-10\% of the cost of $\mathrm{SiGe}$ and will be performing directly in air.

\section{2 Introduction}

The increasing search for new alternatives to supply the demand for energy holds an incredible opportunity to stablish a sustainable energy future. Eco-friendly energy and devices has gained an increased attention; however, these energy alternatives need to be able to compete with existing 
conventional technologies ${ }^{[1,2]}$. Electrical power generation from waste heat is one of the promising eco-friendly technologies due to the excessive amount of energy lost as waste heat. Particularly, TE materials are one of the rising candidates and could contribute to the most prominent energy production for harnessing the waste heat. TE devices hold beneficial features such as not having moving parts, extended longevity, and the ability of convert heat directly into electricity ${ }^{[3-5]}$. The energy conversion efficiency of the TE materials is described by the $Z T$, which is defined as $Z T=$ $S^{2} \rho^{-1} \kappa^{-1} T$, where $S, \rho, S^{2} \rho^{-1}$, and $\kappa$ are the Seebeck coefficient, electrical resistivity, electrical power factor(PF) and thermal conductivity, respectively.

Nowadays, the well-developed TE materials are with high ZT values. Those materials include conventional p-type TE materials available commercially such as bismuth antimony telluride $\left(\mathrm{Bi}_{0.5} \mathrm{Sb}_{1.5} \mathrm{Te}_{3}\right)^{[6]}$ and silicon germanium $\left(\mathrm{Si}_{80} \mathrm{Ge}_{20}\right)^{[7]}$ which hold values of $Z T \sim 1$. However, conventional TE materials are not necessarily eco-friendly, likely limiting the utilization of this materials. Even though the performance of those materials is fascinating, they are usually heavy, toxic, and low in abundance as natural resources, and their thermal or chemical stability are inferiors. Most of all, for those of SiGe and $\mathrm{Yb}_{14} \mathrm{MnSb}_{11}{ }^{[8]}$ that do have superior stability, they only function in the strict oxygen-free environment at high temperatures.

Alternatively, ceramic oxide materials have attracted considerable attention due to its lower cost, lighter weight, non-toxicity, and the capability to overcome thermostability at high temperatures ${ }^{[9]}$. The discovery of the good thermoelectric properties of the layered cobalt oxides in $1997^{[10]}$-first synthesized in $1973^{[11]}$ - exposed the possibility for practical applications for oxides as TE materials. Due to their intrinsic characteristics, layered cobalt oxides have been extremely versatile not only due to their potential in thermoelectric generation ${ }^{[3,4]}$ but also for potential superconducting applications ${ }^{[12]}$ and as a cathode material for batteries ${ }^{[13]}$. The versatility 
lies in the opportunity to vary the amount of electropositive metal separated by the transition metal oxide layers, this allows to alter the electronic and chemical properties over an extensive variety of alternatives ${ }^{[14]}$. As is the case in the perovskite $\mathrm{La}_{1-x} \mathrm{Sr}_{x} \mathrm{CoO}_{3}$, the degeneracy of $3 d$ electrons in cobalt ions is considered to have a decisive role in these layered cobalt oxide materials ${ }^{[15]}$. Particularly, the low spin state of the $\mathrm{Co}^{3+}$ in the $\mathrm{CdI}_{2}$-type $\mathrm{CoO}_{2}$ layer have a huge factor in the large Seebeck coefficient ${ }^{[15,16]}$. There have been numerous research on such layered cobaltites ${ }^{[3,4,}$ 9, 10, 16], in particular, calcium cobaltite $\mathrm{Ca}_{3} \mathrm{Co}_{4} \mathrm{O}_{9}$ has been gaining increasing attention as a $p$-type TE material ${ }^{[3]}$, especially for high temperature applications ${ }^{[17]}$.

Table 9-1 shows the progression of the performance of $\mathrm{Ca}_{3} \mathrm{Co}_{4} \mathrm{O}_{9}$ thermoelectric material ${ }^{[18-}$ 56]. As it can be seen, the $\mathrm{Ca}_{3} \mathrm{Co}_{4} \mathrm{O}_{9}$ single crystals performance offers a competitive TE performance value of $Z T$ of 0.89 at $973 \mathrm{~K}$ for the $\mathrm{Bi}$ doped $\mathrm{Ca}_{3} \mathrm{Co}_{4} \mathrm{O}_{9}$ single crystals ${ }^{[24]}$. However, the main setback for the $\mathrm{Ca}_{3} \mathrm{Co}_{4} \mathrm{O}_{9}$ lays in its low performance for the polycrystalline material ${ }^{[4]}$. Most of the $\mathrm{Ca}_{3} \mathrm{Co}_{4} \mathrm{O}_{9}$ bulk materials reported $Z T$ values $\sim 0.3$ at $\sim 900 \mathrm{~K}^{[3]}$.

The focal challenge of the low $Z T$ value of polycrystalline $\mathrm{Ca}_{3} \mathrm{Co}_{4} \mathrm{O}_{9}$ is the low electrical PF caused by to the low $S$ and high $\rho$. Therefore, to reduce the big gap between the single crystal and the polycrystalline material there is a need to find a consistent improvement in the $Z T$ value of bulk $\mathrm{Ca}_{3} \mathrm{Co}_{4} \mathrm{O}_{9}$. It is important to optimize all aspects of the polycrystalline synthesis process. Previously, the optimal conditions for the calcination ${ }^{[57]}$ and the sintering ${ }^{[51]}$ stages were reported. The sol-gel chemical route is reported to significantly reduce the secondary phase content in $\mathrm{Ca}_{3} \mathrm{Co}_{4} \mathrm{O}_{9}{ }^{[58]}$, since common secondary phases such as Secondary phases such as $\mathrm{Co}_{3} \mathrm{O}_{4}$ has been reported to decrease the performance of the material ${ }^{[59]}$. One of the most significant approaches to boost the TE performance of polycrystalline $\mathrm{Ca}_{3} \mathrm{Co}_{4} \mathrm{O}_{9}$ is the stoichiometric substitution of the $\mathrm{Ca}$ 
Table 9-1: Progression of the performance of $\mathrm{Ca}_{3} \mathrm{Co}_{4} \mathrm{O}_{9}$ thermoelectric material.

\begin{tabular}{|c|c|c|c|c|c|c|c|c|}
\hline Ref & Year & Stoichiometry & $\begin{array}{c}S \\
(\mu \mathrm{V} / \mathrm{K})\end{array}$ & $\begin{array}{c}\rho \\
(\mu \Omega \mathrm{m})\end{array}$ & $\begin{array}{c}\kappa \\
(\mathrm{W} / \mathrm{Km})\end{array}$ & $\begin{array}{c}S^{2} / \rho \\
\left(\mathrm{mW} / \mathrm{K}^{2} \mathrm{~m}\right)\end{array}$ & $Z T$ & $\begin{array}{c}T \\
(\mathrm{~K})\end{array}$ \\
\hline (18) & 2000 & $\mathrm{Ca}_{2.5} \mathrm{Bi}_{0.5} \mathrm{Co}_{4} \mathrm{O}_{9}$ & 160 & 95 & 1.37 & 0.27 & 0.2 & 973 \\
\hline (19) & 2002 & $\mathrm{Ca}_{2.4} \mathrm{Bi}_{0.3} \mathrm{Na}_{0.3} \mathrm{Co}_{4} \mathrm{O}_{9}$ & 204 & 75 & 1.75 & 0.55 & 0.32 & 1000 \\
\hline (20) & 2003 & $\mathrm{Ca}_{3} \mathrm{Co}_{4} \mathrm{O}_{9}$ single crystal & 245 & 23 & 2.90 & 2.61 & 0.87 & 973 \\
\hline (21) & 2004 & $\mathrm{Ca}_{2.7} \mathrm{Eu}_{0.3} \mathrm{Co}_{4} \mathrm{O}_{9}$ & 194 & 78 & 1.65 & 0.48 & 0.30 & 1000 \\
\hline (22) & 2004 & $\mathrm{Ca}_{2.7} \mathrm{D}_{\mathrm{y} 0.3} \mathrm{Co}_{4} \mathrm{O}_{9}$ & 190 & 85 & 1.60 & 0.42 & 0.27 & 1000 \\
\hline (23) & 2005 & $\mathrm{Ca}_{3} \mathrm{Co}_{4} \mathrm{O}_{9} / \mathrm{Ag}-10 \mathrm{wt} \%$ & 175 & 30 & & 1.02 & & 1073 \\
\hline (24) & 2006 & $\mathrm{Ca}_{2.9} \mathrm{Bi}_{0.1} \mathrm{Co}_{4} \mathrm{O}_{9}$ single crystal & 200 & 14.5 & 3.00 & 2.76 & 0.89 & 973 \\
\hline (25) & 2008 & $\mathrm{Ca}_{2.7} \mathrm{Ag}_{0.3} \mathrm{Co}_{4} \mathrm{O}_{9}$ & 229 & 75 & 2.10 & 0.70 & 0.32 & 1000 \\
\hline (26) & 2008 & $\mathrm{Ca}_{2.7} \mathrm{Gd}_{0.3} \mathrm{Co}_{4} \mathrm{O}_{9}$ & 182 & 78 & 1.40 & 0.42 & 0.24 & 973 \\
\hline (27) & 2009 & $\mathrm{Ca}_{2.7} \mathrm{Ag}_{0.3} \mathrm{Co}_{4} \mathrm{O}_{9} / \mathrm{Ag}-10 \mathrm{wt} \%$ & 218 & 37 & 2.50 & 1.28 & 0.50 & 1000 \\
\hline (28) & 2009 & $\mathrm{Ca}_{2.7} \mathrm{Y}_{0.3} \mathrm{Co}_{4} \mathrm{O}_{9}$ & 172 & 85 & 1.55 & 0.35 & 0.22 & 973 \\
\hline (29) & 2010 & $\mathrm{Ca}_{3} \mathrm{Co}_{3.7} \mathrm{Ti}_{0.3} \mathrm{O}_{9}$ & 285 & 165 & 1.80 & 0.49 & 0.29 & 1000 \\
\hline (30) & 2010 & $\mathrm{Ca}_{3} \mathrm{Co}_{3.9} \mathrm{Fe}_{0.1} \mathrm{O}_{9}$ & 258 & 97 & 1.78 & 0.69 & 0.39 & 1000 \\
\hline (31) & 2010 & $\mathrm{Ca}_{3} \mathrm{Co}_{3.95} \mathrm{Ga}_{0.05} \mathrm{Co}_{9}$ & 200 & 77 & 1.50 & 0.52 & 0.36 & 1073 \\
\hline (32) & 2011 & $\mathrm{Ca}_{2.97} \mathrm{Ag}_{0.03} \mathrm{Co}_{4} \mathrm{O}_{9}$ & 200 & 89 & 2.10 & 0.45 & 0.23 & 973 \\
\hline (33) & 2011 & $\mathrm{Ca}_{2.7} \mathrm{Er}_{0.3} \mathrm{Co}_{4} \mathrm{O}_{9}$ & 192 & 100 & 1.42 & 0.37 & 0.28 & 1073 \\
\hline (34) & 2011 & $\mathrm{Ca}_{2.8} \mathrm{Lu}_{0.2} \mathrm{Co}_{4} \mathrm{O}_{9}$ & 194 & 95 & 1.20 & 0.40 & 0.36 & 1073 \\
\hline (35) & 2011 & $\mathrm{Ca}_{2.8} \mathrm{Ag}_{0.05} \mathrm{Lu}_{0.15} \mathrm{Co}_{4} \mathrm{O}_{9}$ & 235 & 72 & 1.40 & 0.77 & 0.61 & 1118 \\
\hline (36) & 2011 & $\mathrm{Ca}_{2.8} \mathrm{Pr}_{0.2} \mathrm{Co}_{4} \mathrm{O}_{9}$ & 201 & 107 & 1.55 & 0.38 & 0.24 & 973 \\
\hline$(37)$ & 2014 & $\mathrm{Ca}_{3} \mathrm{Co}_{3.85} \mathrm{Cr}_{0.15} \mathrm{O}_{9}$ & 195 & 110 & 2.00 & 0.35 & 0.19 & 1073 \\
\hline$(38)$ & 2014 & $\mathrm{Ca}_{2.7} \mathrm{Bi}_{0.3} \mathrm{Co}_{3.9} \mathrm{Fe}_{0.1} \mathrm{O}_{9}$ & 182 & 73 & 1.19 & 0.45 & 0.37 & 973 \\
\hline (39) & 2014 & $\mathrm{Ca}_{2 .} \mathrm{La}_{0.1} \mathrm{Co}_{3.9} \mathrm{Fe}_{0.1} \mathrm{O}_{9}$ & 226 & 125 & 1.29 & 0.41 & 0.32 & 1000 \\
\hline (40) & 2014 & $\mathrm{Ca}_{3} \mathrm{Co}_{3.9} \mathrm{Cd}_{0.1} \mathrm{O}_{9}$ & 209 & 82 & 1.50 & 0.53 & 0.35 & 1000 \\
\hline$(41)$ & 2015 & $\mathrm{Ca}_{3} \mathrm{Co}_{4} \mathrm{O}_{9}$ & 132 & 184 & 0.41 & 0.12 & 0.29 & 1000 \\
\hline$(42)$ & 2015 & $\mathrm{Ca}_{3} \mathrm{Ba}_{0.05} \mathrm{Co}_{4} \mathrm{O}_{9}$ & 185 & 41 & 1.70 & 0.83 & 0.52 & 1073 \\
\hline$(43)$ & 2016 & $\mathrm{Ca}_{2.8} \mathrm{Ba}_{0.1} \mathrm{Pr}_{0.1} \mathrm{Co}_{4} \mathrm{O}_{9}$ & 207 & 92 & 1.65 & 0.47 & 0.31 & 973 \\
\hline (44) & 2016 & $\mathrm{Ca}_{3} \mathrm{Co}_{4} \mathrm{O}_{9}+5$ wt. $\% \mathrm{~K}_{2} \mathrm{CO}_{3}$ & 230 & 125 & 1.32 & 0.42 & 0.35 & 1073 \\
\hline (45) & 2016 & $\mathrm{Ca}_{2.8} \mathrm{Bi}_{0.2} \mathrm{Co}_{4} \mathrm{O}_{9}$ & 192 & 50 & 1.90 & 0.74 & 0.43 & 1073 \\
\hline (46) & 2016 & $\mathrm{Ca}_{3} \mathrm{Co}_{4} \mathrm{O}_{9}$ & 170 & 175 & 0.49 & 0.17 & 0.31 & 900 \\
\hline (47) & 2017 & $\mathrm{Ca}_{2.55} \mathrm{Na}_{0.45} \mathrm{Co}_{4} \mathrm{O}_{8.55} \mathrm{~F}_{0.45}$ & 192 & 130 & 2.20 & 0.28 & 0.13 & 873 \\
\hline (48) & 2017 & $\mathrm{Ca}_{3} \mathrm{Co}_{4} \mathrm{O}_{9}$ & 210 & 171 & 0.63 & 0.26 & 0.40 & 1073 \\
\hline (49) & 2017 & $\mathrm{Ca}_{2.5} \mathrm{~Tb}_{0.5} \mathrm{Co}_{4} \mathrm{O}_{9}$ & 323 & 91 & 1.25 & 1.15 & 0.74 & 800 \\
\hline$(50)$ & 2017 & $\mathrm{Ca}_{2.9} \mathrm{Bi}_{0.1} \mathrm{Ba}_{0.07} \mathrm{Co}_{4} \mathrm{O}_{9}$ & 190 & 40 & & 0.90 & & 1073 \\
\hline$(51)$ & 2018 & $\mathrm{Ca}_{3} \mathrm{Co}_{4} \mathrm{O}_{9} \mathrm{~K}_{0.1}$ & 190 & 44 & & 0.82 & & 1073 \\
\hline (52) & 2018 & $\mathrm{Ca}_{2.95} \mathrm{Na}_{0.05} \mathrm{Co}_{3.975} \mathrm{~W}_{0.025} \mathrm{O}_{9}$ & 183 & 130 & 1.26 & 0.26 & 0.21 & 1000 \\
\hline (53) & 2018 & $\mathrm{Ca}_{3} \mathrm{Co}_{4} \mathrm{O}_{9}$ & 189 & 72 & 1.95 & 0.50 & 0.28 & 1073 \\
\hline (54) & 2019 & $\mathrm{Ca}_{2.25} \mathrm{Na}_{0.3} \mathrm{Bi}_{0.35} \mathrm{~Tb}_{0.1} \mathrm{Co}_{4} \mathrm{O}_{9}$ & 236 & 86 & 1.96 & 0.65 & 0.35 & 1073 \\
\hline (55) & 2019 & $\mathrm{Ca}_{2.97} \mathrm{Sr}_{0.03} \mathrm{Co}_{4} \mathrm{O}_{9}$ & 275 & 65 & 4.40 & 1.16 & 0.29 & 1073 \\
\hline (56) & 2019 & $\mathrm{Ca}_{2.25} \mathrm{Na}_{0.3} \mathrm{Bi}_{0.35} \mathrm{~Tb}_{0.1} \mathrm{Co}_{4} \mathrm{O}_{9}$ & 240 & 70 & & 0.82 & & 1073 \\
\hline$*$ & 2019 & $\mathrm{Ca}_{2.95} \mathrm{~Tb}_{0.05} \mathrm{Co}_{4} \mathrm{O}_{9} \mathrm{Bi}_{0.25}$ & 212 & 36 & 1.52 & 1.25 & 0.89 & 1073 \\
\hline
\end{tabular}

$\operatorname{sites}^{[3,4,9,18,19,21-23,25-41,43,44,48-50,52-56]}$. Additionally, non-stoichiometric addition has been reported to greatly enhance the TE performance ${ }^{[41,43,48,53]}$. Those approaches can be used to overcome the big gap to compete with the performance of conventional p-type TE materials and that of the $\mathrm{Ca}_{3} \mathrm{Co}_{4} \mathrm{O}_{9}$ single crystal. TE energy conversion efficiency of polycrystalline $\mathrm{Ca}_{3} \mathrm{Co}_{4} \mathrm{O}_{9-\delta}$ 
ceramics is currently only about $\sim 30-60 \%$ of that of the single crystals. Furthermost, the improvement on the TE performance of the material proposed in this work makes the $\mathrm{Ca}_{3} \mathrm{Co}_{4} \mathrm{O}_{9}$ an encouraging candidate to compete against conventional and emerging p-type thermoelectric materials. The $\mathrm{Ca}_{3} \mathrm{Co}_{4} \mathrm{O}_{9}$ is closer than ever to surpass a $\mathrm{ZT}$ value of one, as shown in Figure 9-1.

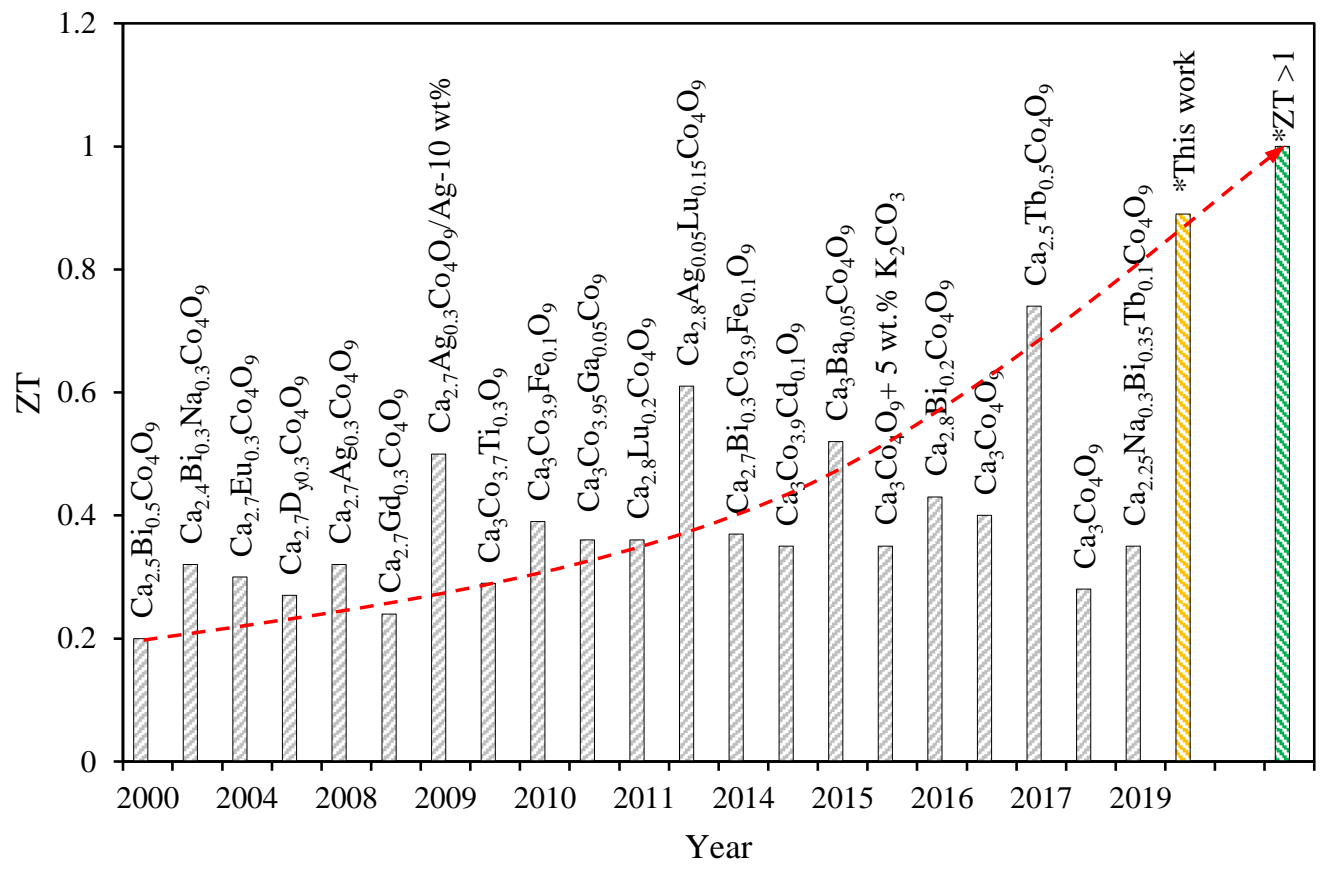

Figure 9-1: Timeline of the improvement in the $\mathrm{ZT}$ of $\mathrm{Ca}_{3} \mathrm{Co}_{4} \mathrm{O}_{9}$ polycrystalline materials by the addition of different doping elements. ${ }^{(18,19,21,22,25-27,29-31,34,35,38,40,42,44,45,48,49,53,54)}$. *ZT value of this work is also reported. The goal of reaching a ZT material over 1 for commercial applications would be achievable within a few years.

In this work, we report the first time $\mathrm{Ca}_{3} \mathrm{Co}_{4} \mathrm{O}_{9}$ ceramics surpass the $\mathrm{ZT}$ of the single crystal by using a systematic dual doping technique with simultaneous non-stoichiometric addition of Bismuth (Bi) and stoichiometric substitution of Terbium $(\mathrm{Tb})$ for Calcium $(\mathrm{Ca})$ to enhance the microstructure and thermoelectric performance of misfit layered calcium cobaltite $\mathrm{Ca}_{3} \mathrm{Co}_{4} \mathrm{O}_{9}$ ceramics. The designed nominal chemistry of the polycrystalline ceramic samples in this chapter 
are that of the undoped $\mathrm{Ca}_{3} \mathrm{Co}_{4} \mathrm{O}_{9}$ and the dual doped $\mathrm{Ca}_{2.95} \mathrm{~Tb}_{0.05} \mathrm{Co}_{4} \mathrm{O}_{9} \mathrm{Bi}_{\mathrm{y}}(y=0,0.10,0.15,0.20$, 0.25 , and 0.30$)$.

To understand the dual doping approach used to obtain the best ZT of the polycrystalline $\mathrm{Ca}_{3} \mathrm{Co}_{4} \mathrm{O}_{9}$, we first reported the structure and $\mathrm{TE}$ properties of $\mathrm{Tb}$ in a previous chapter followed by the addition of the second dopant in this chapter after the optimized first iteration. Using this systematic approach, the effect of a dual doping with simultaneous stoichiometric substitution of $\mathrm{Tb}$ in the $\mathrm{Ca}$ sites and non-stoichiometric addition of $\mathrm{Bi}$ into the $\mathrm{Ca}_{3} \mathrm{Co}_{4} \mathrm{O}_{9}$ is reported. Previous reports showed that the dual doping produces a selective segregation at the GBs and that the main driving force for the segregation is linked to the strain effect, in which bigger over-sized atoms segregate to the GBs, displacing smaller-sized atoms to the interior of the crystal lattice ${ }^{[48]}$. In the material studied, $\mathrm{Bi}$ is the over-sized atom in comparison to $\mathrm{Tb}$ and the host ions, thus, $\mathrm{Bi}$ is segregated to the GBs. For single dopants, GBs segregation have been explored to studied the influence on the $\mathrm{Bi}^{3+}$ as substitution in the $\mathrm{Ca}^{2+}$ site $e^{[43]}$, the non-stoichiometric addition of $\mathrm{Ba}^{2+[41]}$, and the non-stoichiometric addition of $\mathrm{K}^{+[53]}$. To further understand the influence of the segregation in a dual doping approach and their impact on the nanostructure and TE performance of doped $\mathrm{Ca}_{3} \mathrm{Co}_{4} \mathrm{O}_{9}$, the simultaneous stoichiometric substitution of $\mathrm{Tb}$ in the $\mathrm{Ca}$ sites and the nonstoichiometric addition of Bi was investigated.

The chemistry analysis explored under TEM revealed the segregation of the $\mathrm{Bi}$ at the Grain Boundaries (GBs) and showed depletion of $\mathrm{Tb}$ at the $\mathrm{GBs}$ of $\mathrm{Ca}_{3} \mathrm{Co}_{4} \mathrm{O}_{9}$ grains with different $\mathrm{c}-$ axis. Additionally, TEM results exposed $\mathrm{Bi}$ is present in inferior quantities and $\mathrm{Tb}$ content is higher at the $\mathrm{Ca}_{3} \mathrm{Co}_{4} \mathrm{O}_{9}$ lattice than the GBs concentration of each respective element. Systematically optimizing the amount of $\mathrm{Tb}$ previously, and later adding Bi non-stoichiometric addition to the best $\mathrm{Tb}$ concentration enhances the Seebeck Coefficient and decreases the electrical resistivity 
simultaneously which resulted in a high electrical power factor and low thermal conductivity. Crystal growth, grain alignment improvement and enhanced crystal texture of the micron sized crystal grains is possible with the dual element doping. Low thermal conductivity is induced by the incorporation of both heavy doping elements due to decrease in the lattice thermal contribution. Combined with a high electrical power factor and low thermal conductivity $\mathrm{Ca}_{2.95} \mathrm{~Tb}_{0.05} \mathrm{Co}_{4} \mathrm{O}_{9} \mathrm{Bi}_{0.25}$ produced a record thermoelectric ZT of $\sim 0.89$ at $1073 \mathrm{~K}$ which is comparable to current state-ofthe-art high temperature p-type thermoelectric materials. The obtained high performance might be the start of suitable oxide ceramics for thermoelectric commercial applications. The techniques and effective engineering approaches to increase the performance of bulk ceramics $\mathrm{Ca}_{3} \mathrm{Co}_{4} \mathrm{O}_{9-\delta}$, to significantly outperform $\mathrm{Ca}_{3} \mathrm{CO}_{4} \mathrm{O}_{9-\delta}$ single crystals, by introducing dual dopants for designing the grain boundaries and interfaces in polycrystalline ceramics is reported.

\section{3 Experimental section}

Instead of using the special techniques such as Spark Plasma Sintering, the present work applied conventional cold pressing and sintering to make the oxide pellets using the powders synthesized through the chemical sol-gel route, to precisely control the stoichiometry and dopants distribution with strict uniformity. The polycrystalline ceramic samples were prepared by the conventional chemical sol-gel route with a chemical composition of the undoped $\mathrm{Ca}_{3} \mathrm{Co}_{4} \mathrm{O}_{9}$ and the dual doped $\mathrm{Ca}_{2.95} \mathrm{~Tb}_{0.05} \mathrm{Co}_{4} \mathrm{O}_{9} \mathrm{Bi}_{\mathrm{y}}(y=0,0.10,0.15,0.20,0.25$, and 0.30$)$. The precursor powders were obtained mixing chemical reagents: $\mathrm{Ca}\left(\mathrm{NO}_{3}\right)_{2} \cdot 4 \mathrm{H}_{2} \mathrm{O}$ (99\%, Acros Organics), $\mathrm{Co}\left(\mathrm{NO}_{3}\right)_{2} \cdot 6 \mathrm{H}_{2} \mathrm{O}$ (99\%, Acros Organics), $\mathrm{Bi}\left(\mathrm{NO}_{3}\right)_{3} \cdot 5 \mathrm{H}_{2} \mathrm{O}$ (98\%, Acros Organics), and $\mathrm{Tb}\left(\mathrm{NO}_{3}\right)_{3} \cdot 6 \mathrm{H}_{2} \mathrm{O}(99.9 \%$ Strem Chemicals) in deionized water according to the stoichiometric chemical compositions. Citric acid (BDH Chemical), ethylene glycol, and polyethylene glycol were dissolved in the solution to polymerize the mixture. Nitric acid was included to the solution 
to promote the sol-gel synthesis, nitrate salts decomposition, and the new compound formation. The sol-gel mixture was mechanically stirred at $353 \mathrm{~K}$ for $3 \mathrm{~h}$. The obtained sol-gel mixture was ashed at $773 \mathrm{~K}$ for $2 \mathrm{hr}$ in air inside a box furnace. The subsequent ashes were ball-milled in ethyl alcohol in a stage with zirconia balls and then dried at room temperature with a final manually ground stage after that to obtain uniform grain size in the powder. The reasonable homogeneous ashes were calcined at $973 \mathrm{~K}$ for $4 \mathrm{~h}$ in a tube furnace with a constant oxygen flow. The calcined powders were uniaxially pressed into round pellets under a 1 Gpa pressure at room temperature. The bulk samples were sintered at $1233 \mathrm{~K}$ in a tube furnace with a constant oxygen flow and then cut into rectangular pellets to obtain the final desired sample for either the electrical or thermal measurements. The apparent densities for all bulk samples are listed in Table 9-2.

Table 9-2: Apparent density of the $\mathrm{Ca}_{3} \mathrm{Co}_{4} \mathrm{O}_{9}$ samples with dual dopants.

\begin{tabular}{ll}
\hline $\mathrm{Ca}_{3-\mathrm{x}} \mathrm{Tb}_{\mathrm{x}} \mathrm{Co}_{4} \mathrm{O}_{9} \mathrm{Bi}_{\mathrm{y}}$ & Average Apparent density $(\mathrm{g} / \mathrm{cm} 3)$ \\
\hline $\mathrm{x}=0 \quad \mathrm{y}=0$ & 3.883 \\
$\mathrm{x}=0.05 \mathrm{y}=0$ & 4.182 \\
$\mathrm{x}=0.05 \mathrm{y}=0.10$ & 3.911 \\
$\mathrm{x}=0.05 \mathrm{y}=0.15$ & 3.909 \\
$\mathrm{x}=0.05 \mathrm{y}=0.20$ & 3.970 \\
$\mathrm{x}=0.05 \mathrm{y}=0.25$ & 4.295 \\
$\mathrm{x}=0.05 \mathrm{y}=0.30$ & 4.701 \\
\hline
\end{tabular}

The electrical resistivity $(\rho)$ and absolute Seebeck coefficient $(S)$ were measured at the same time by the four-terminal method using a Linseis LSR-1100 unit. The measurements were performed perpendicular to the pressed direction from $323 \mathrm{k}$ to $1073 \mathrm{~K}$ under a low pressure He environment. The thermal conductivity was calculated using the equation $\kappa=\lambda C_{p} \rho m$, where $C_{p}, \lambda$, and $m$ are the specific heat, thermal diffusivity, and mass density, respectively. The $C p$ and $\lambda$ values were obtained in the range 323 to $1073 \mathrm{~K}$ using a Linseis Laser Flash Analyzer 1200. The measurements were also performed perpendicular to the pressed direction and samples were 
analyzed under a low-pressure air environment. X-Ray powder Diffraction (XRD) analysis was performed using a PANatycal X'Pert Pro XRD unit for crystal phase and lattice parameter determination purposes. The ground powders from the sintered samples were used in the XRD analysis using $\mathrm{Cu} \mathrm{K}$-alpha radiation, $45 \mathrm{kV}$ voltage and $40 \mathrm{~mA}$ current at room temperature. The cross-section and plan-view morphology of the sintered pellets was observed using a HitachiS4700F Scanning Electron Microscope (SEM) unit. Transmission electron microscopy (TEM) samples were prepared from sintered pellets by mechanically polishing and ion milling in a liquidnitrogen-cooled holder. TEM imaging including Energy dispersive spectroscopy (EDS) and high resolution TEM were performed in a JEM-2100 operated at $200 \mathrm{kV}$. X-ray Photoelectron Spectroscopy (XPS) was performed using Physical Electronics PHI 5000 VersaProbe system. Multipak XPS software was used to analyze the obtained data.

\section{4 Results}

The temperature dependence of the electrical properties are shown in Figure 9-2(a) $\rho-T$, (b) $S$ $T$, and (c) $S^{2} / \rho-T$ for different $\mathrm{Ca}_{3-\mathrm{x}} \mathrm{Tb}_{\mathrm{x}} \mathrm{Co}_{4} \mathrm{O}_{9} \mathrm{Bi}_{\mathrm{y}}$ doping levels. Inset in Figure 9-2(a) shows the direction in which the electrical measurements were performed for $\rho-T, \mathrm{~S}-T$, and $S^{2} / \rho-T$. The $\rho$-T properties of $\mathrm{Ca}_{2.95} \mathrm{~Tb}_{0.05} \mathrm{Co}_{4} \mathrm{O}_{9}$ have a metallic-like behavior and a transition to a semiconductorlike behavior around $473 \mathrm{~K}$, reaching a $\rho$ slightly lower in magnitude to the pristine material. In contrast, the $\rho$-T curves in Figure 9-2 (a) for $\mathrm{Ca}_{3-\mathrm{x}} \mathrm{Tb}_{\mathrm{x}} \mathrm{Co}_{4} \mathrm{O}_{9} \mathrm{Bi}_{\mathrm{y}}$ show a metallic-like behavior and the $\rho$ increases as the temperature increases, except for the concentration $y=0.30$ which shows a semiconductor-like behavior and higher $\rho$ values. With the increase in Bi doping level up to $y=0.25$ the magnitude of $\rho$ decreases notably. The best performing dual doping concentration $\mathrm{Ca}_{2.95} \mathrm{~Tb}_{0.05} \mathrm{Co}_{4} \mathrm{O}_{9} \mathrm{Bi}_{0.25}$ reaches the lowest $\rho$ value of $13.2 \mu \Omega \mathrm{m}$ at $310 \mathrm{~K}$ which correspond to a significant reduction in comparison with that of the baseline calcium cobaltite. The meaningfully 
(a)

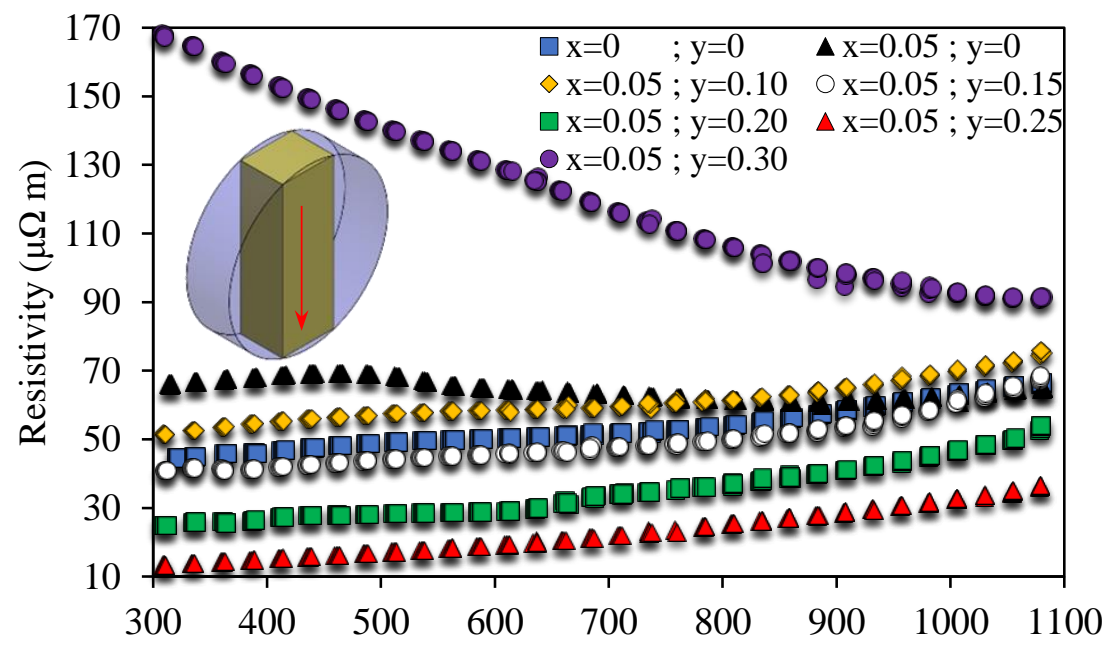

(b)

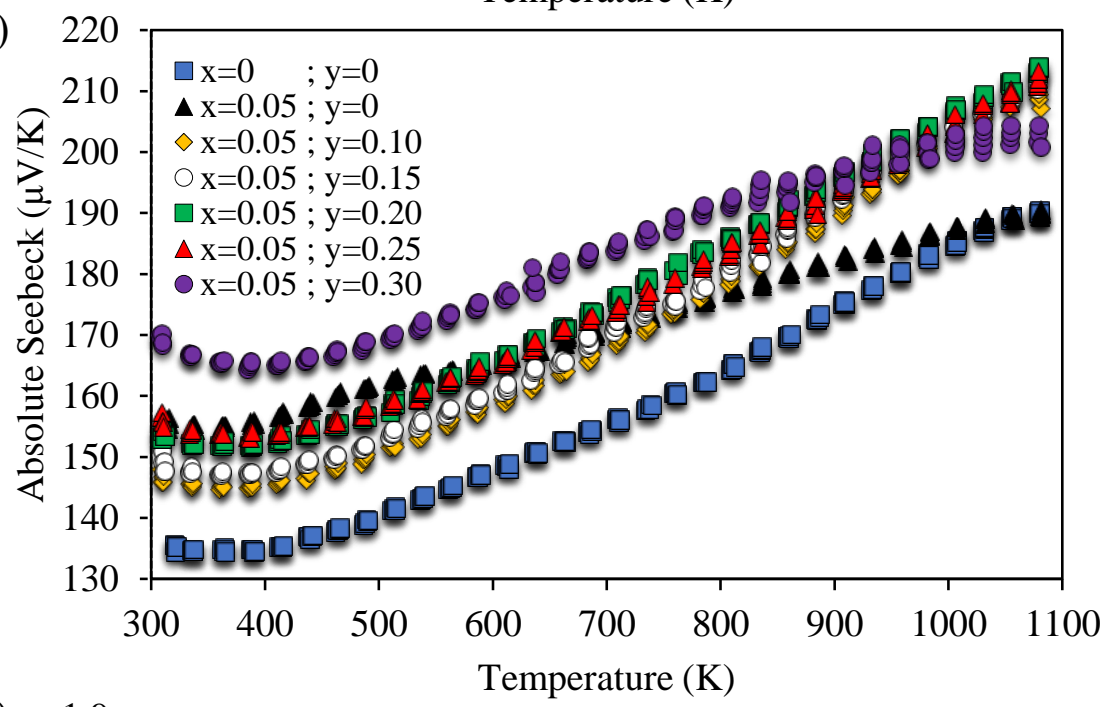

(c)

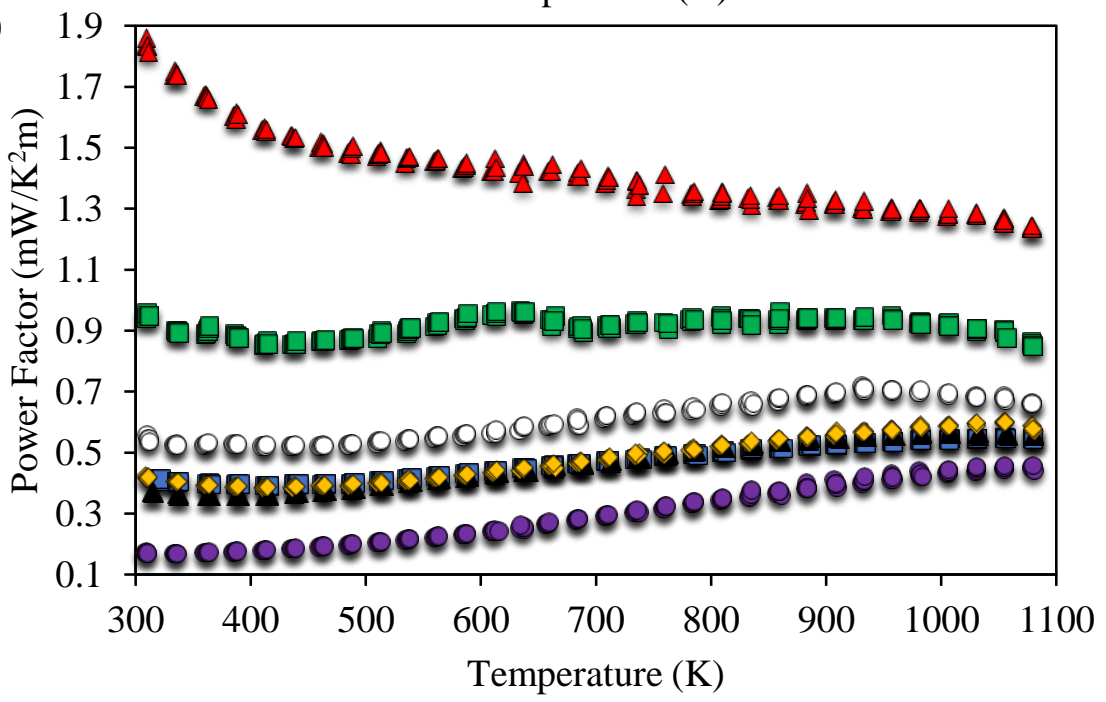

Figure 9-2: (a) $\rho$-T, (b) $S$-T, and (c) $S^{2} / \rho$-T show the temperature dependence of properties for $\mathrm{Ca}_{3} \mathrm{Co}_{4} \mathrm{O}_{9}$ with dual dopants. Inset figure(a) shows the direction of the performed measurements. 
low $\rho$ could be attributed for the increased carrier mobility in the system and by the nano and micron size crystal orientation improvement by the dual doping approach -as SEM and TEM images show-.

There is a simultaneous impact on the $\mathrm{S}$ by the doping of $\mathrm{Tb}$ into $\mathrm{Ca}_{3} \mathrm{Co}_{4} \mathrm{O}_{9}$. As the $\mathrm{Tb}$ doping level increases the magnitude of the $S$ is increased in the broad temperature range, as shown in the previous chapter. Compared to the pristine $\mathrm{Ca}_{3} \mathrm{CO}_{4} \mathrm{O}_{9}$ ceramics, doping $\mathrm{Tb}$ is a notably enhancer of S even with a small doping level. However, as stated in the $\rho$-T curves for Tb doping suffers of elevated $\rho$ values. With the inclusion of $\mathrm{Bi}$ at $\mathrm{Ca}_{2.95} \mathrm{~Tb}_{0.05} \mathrm{Co}_{4} \mathrm{O}_{9} \mathrm{Bi}_{0.25}$ not only the $\rho$ values were lowered, but also the $\mathrm{S}$ values were preserved at low temperature and most notably it was greatly improved at high temperature in comparison to the pristine $\mathrm{Ca}_{3} \mathrm{Co}_{4} \mathrm{O}_{9}$ and the $\mathrm{Tb}$ at $\mathrm{x}=0.05$ doping level, as shown in Figure 9-2 (b). There is an additional increase of $S$ for the doping level $y=0.3$ at the low temperature regime, nonetheless this improvement is completely diminished above $950 \mathrm{~K}$.

The electrical PF for $\mathrm{Ca}_{2.95} \mathrm{~Tb}_{0.05} \mathrm{Co}_{4} \mathrm{O}_{9}$ shows that a doping level of $x=0.05$ is beneficial to the material since a further increase in the Tb doping level produces a lower PF than the initial baseline, as shown in the previous chapter. Thus, $\mathrm{Ca}_{3-\mathrm{x}} \mathrm{Tb}_{\mathrm{x}} \mathrm{Co}_{4} \mathrm{O}_{9}$ at a doping level of $x=0.05$ was selected a starting point to add a second dopant due to its greater performance. Perhaps, making a good practice finding an optimization for the first dopant before adding a second dopant. For the second dopant, there was a gradual increase of the PF with the increase of Bi up to a doping level of $y=0.25$. The PF was increased with the integration of both outstanding improvements for $\mathrm{Ca}_{2.95} \mathrm{~Tb}_{0.05} \mathrm{Co}_{4} \mathrm{O}_{9} \mathrm{Bi}_{0.25}$, the lowest $\rho$ value and the enhanced $\mathrm{S}$, as shown in Figure 9-2 (c). At 310 $\mathrm{K}$, the electrical PF for the best concentration achieves one of the highest reported value at the room temperature regime for the $\mathrm{Ca}_{3} \mathrm{Co}_{4} \mathrm{O}_{9}$ with a value of $1.83 \mathrm{mWm}^{-1} \mathrm{~K}^{-2}$. At high temperature regime the best performing material maintains an exceptional $\mathrm{PF}$ value of $1.23 \mathrm{mWm}^{-1} \mathrm{~K}^{-2}$. Such 
superior performance makes this material candidate for a wide temperature range application. Nonetheless, there is a saturation limit of the thermoelectric properties, and $\mathrm{Ca}_{2.95} \mathrm{~Tb}_{0.05} \mathrm{Co}_{4} \mathrm{O}_{9} \mathrm{Bi}_{0.30}$ samples show a decreased PF performance.

The thermal conductivity and $Z T$ of the ceramics analyzed are shown in Figure 9-3 (a) $\kappa-T$, (b) $\kappa_{\mathrm{e}}-T$ and $\kappa_{\mathrm{i}}-T$, and (c) $Z T$ for different $\mathrm{Ca}_{3-\mathrm{x}} \mathrm{Tb}_{\mathrm{x}} \mathrm{Co}_{4} \mathrm{O}_{9} \mathrm{Bi}_{\mathrm{y}}$ doping levels. The $\mathrm{Tb}$ concentration level plays a fundamental role in the thermal properties of the $\mathrm{Ca}_{3} \mathrm{Co}_{4} \mathrm{O}_{9}$ samples. With the substitution of $\mathrm{Ca}$ by heavier atoms of $\mathrm{Tb}$ the magnitude of $\kappa$ is continuously reduced with the increasing $\mathrm{Tb}$ doping level, as shown in previous chapter. The cause of the distinctively low $\kappa$ is due to the gradual reduction of both $\kappa_{e}$ and $\kappa_{i}$ contribution by increasing Tb doping level. The nonstoichiometric addition of Bi doping level also plays an important part in the thermal properties of the $\mathrm{Ca}_{3-\mathrm{x}} \mathrm{Tb}_{\mathrm{x}} \mathrm{Co}_{4} \mathrm{O}_{9} \mathrm{Bi}_{\mathrm{y}}$ samples. The addition of $\mathrm{Bi}$ as a second dopant further reduces the $\kappa_{\mathrm{i}}$ contribution, as shown in Figure 9-3 (b). There is a constant decrease in the $\kappa_{\mathrm{i}}$ contribution with the increasing Bi doping level up to $y=0.25$, however, the $\kappa_{\mathrm{e}}$ contribution is also gradually increased with the addition of Bi up to $y=0.25$. For the doping level $y=0.3$ the $\kappa_{\mathrm{i}}$ contribution is deteriorated but still lower than the pristine $\mathrm{Ca}_{3} \mathrm{CO}_{4} \mathrm{O}_{9}$, and the $\kappa_{\mathrm{e}}$ contribution is reduced notably due to the poor electrical performance for $y=0.3$. The contribution of the $\kappa_{\mathrm{e}}$ and $\kappa_{\mathrm{i}}$ gives the $\mathrm{Ca}_{2.95} \mathrm{~Tb}_{0.05} \mathrm{Co}_{4} \mathrm{O}_{9} \mathrm{Bi}_{\mathrm{y}}$ samples a characteristic $\kappa$ total with a non-consistent trend. First a reduction of $\kappa$ for $y=0.10$, then a gradual increase of $\kappa$ with the increasing Bi doping level up to $y=0.25$, and finally a reduction of the overall $\kappa$ for the $y=0.3$ doping level.

The figure of merit is enhanced slightly with the stoichiometric substitution of $\mathrm{Tb}$, resulting in a $Z T$ of 0.38 at $1073 \mathrm{~K}$, achieved only by a small $\mathrm{Tb}$ concentration. The improvement of the $Z T$ by the single dopant is due to the improved $S$ and the reduction of $\kappa$. Complemented by the 
(a)

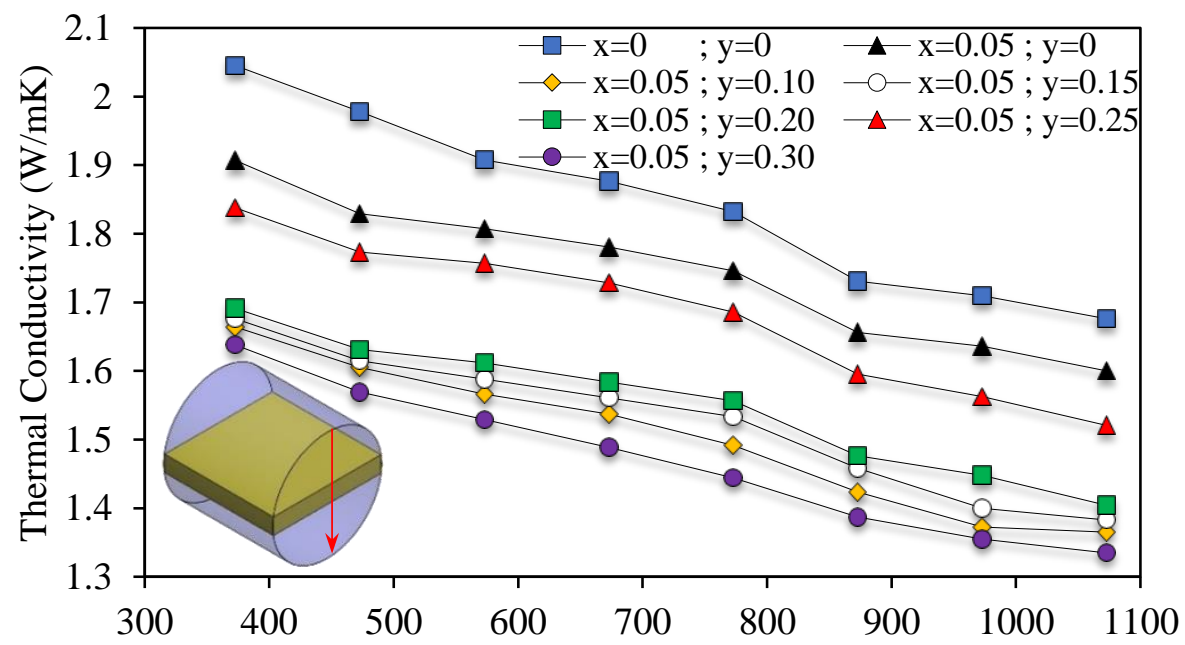

(b)

Temperature $(\mathrm{K})$
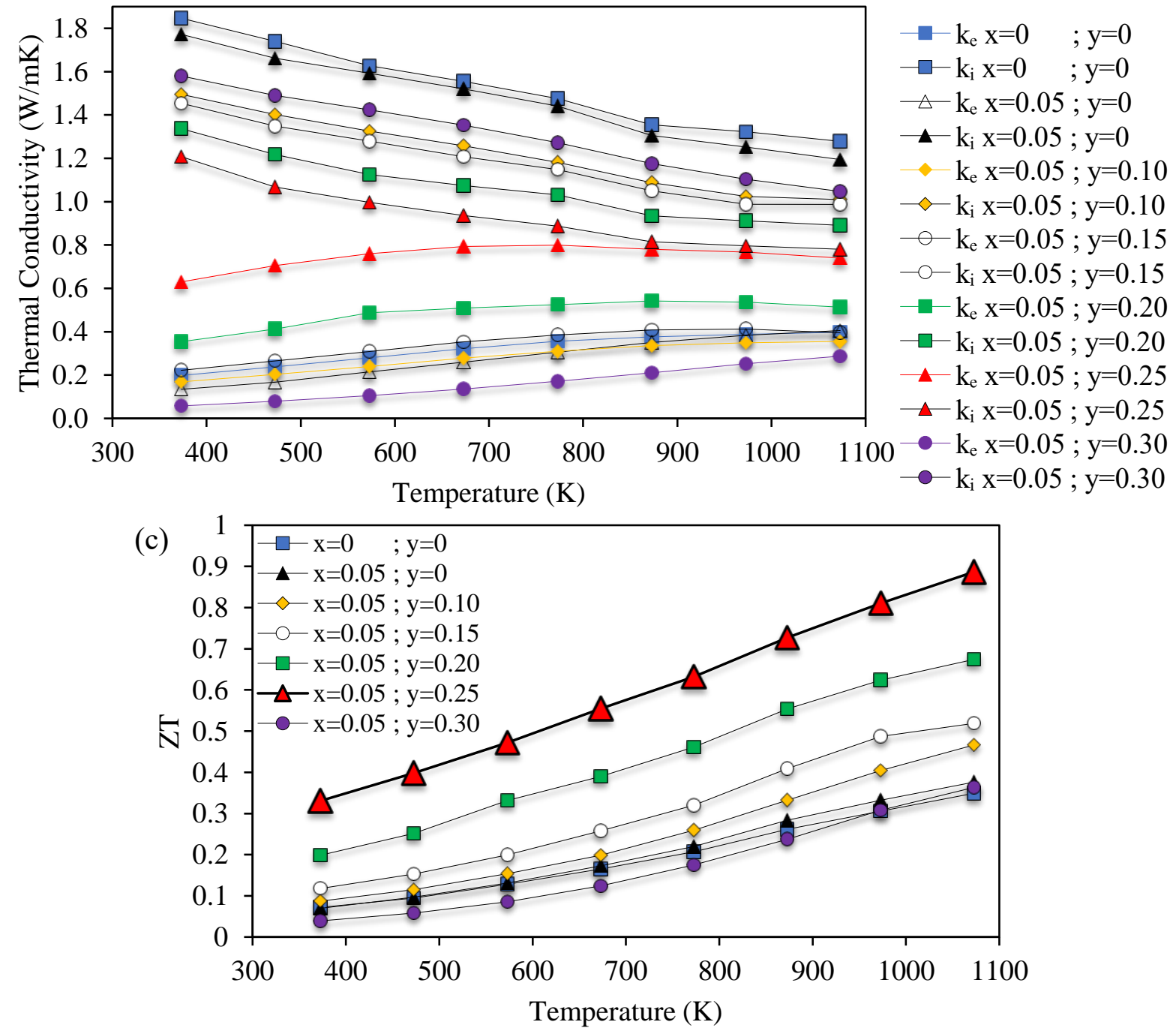

Figure 9-3: Temperature dependence of: (a) total thermal conductivity $(\kappa-T),(b)$ electronic contribution $\left(\kappa_{e}\right)$ and lattice contribution $\left(\kappa_{i}\right)$, and (c) $\mathrm{ZT}$ for $\mathrm{Ca}_{3} \mathrm{Co}_{4} \mathrm{O}_{9}$ with dual dopants. Inset figure in (a) shows the direction of the performed measurements for $\kappa-T$. 
pronounced simultaneous reduction of $\rho$ and improved $S$, the dual non-stoichiometric addition of $\mathrm{Bi}$ and stoichiometric substitution of $\mathrm{Tb}$ reduces the thermal conductivity $\kappa$ in comparison to the pristine $\mathrm{Ca}_{3} \mathrm{Co}_{4} \mathrm{O}_{9}$. Thus, the $Z T$ is outstandingly enhanced for the dual doping approach, resulting in a ZT of $\sim 0.89$ at $1073 \mathrm{~K}$, reached by the $\mathrm{Ca}_{2.95} \mathrm{~Tb}_{0.05} \mathrm{Co}_{4} \mathrm{O}_{9} \mathrm{Bi}_{0.25}$ ceramics.

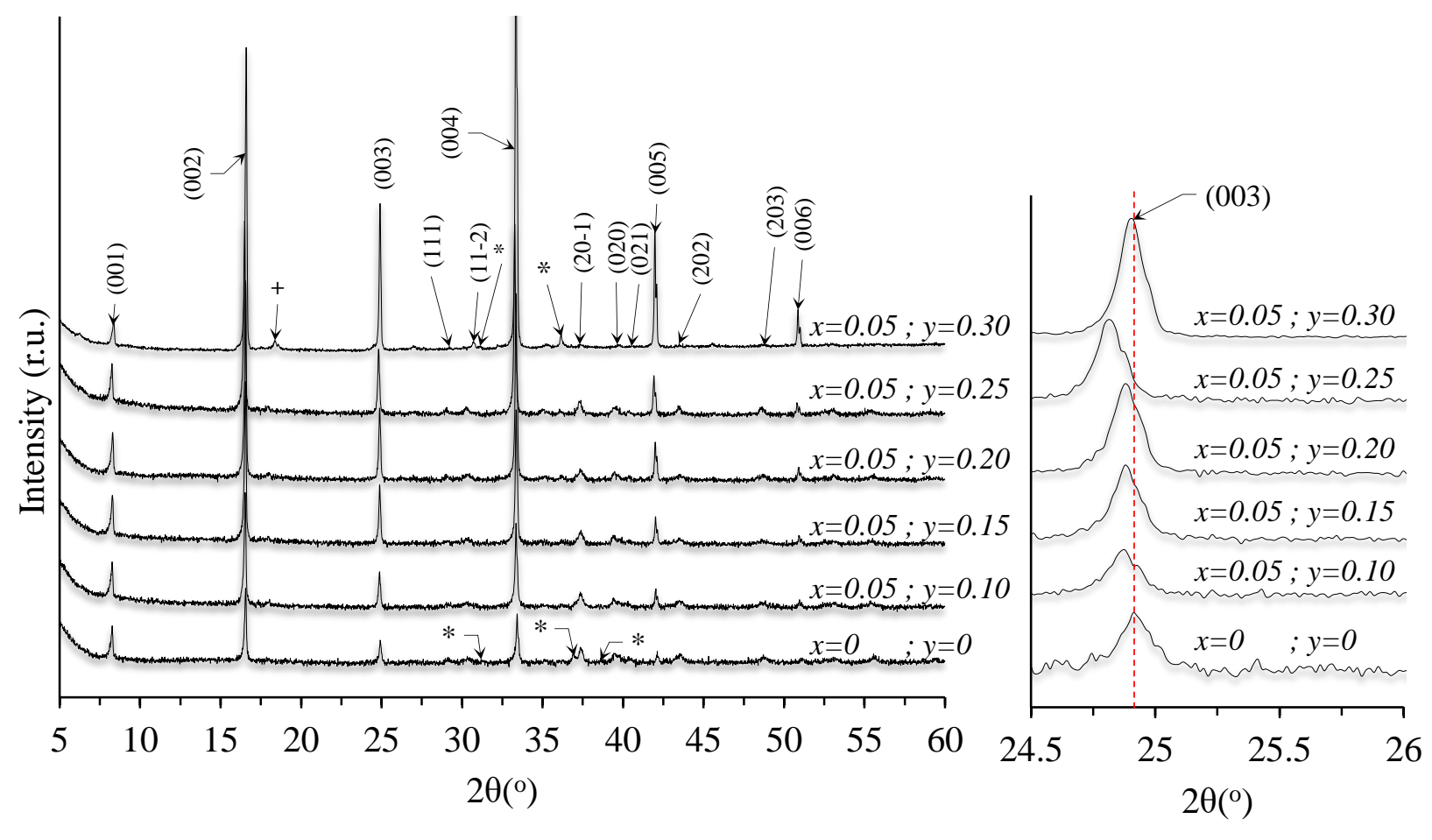

Figure 9-4: XRD powder diffraction patterns for the undoped and the doped $\mathrm{Ca}_{3} \mathrm{CO}_{4} \mathrm{O}_{9}$ samples. In all samples, monoclinic $\mathrm{Ca}_{3} \mathrm{Co}_{4} \mathrm{O}_{9}$ was observed. Nonetheless, a minor amount of $\mathrm{Co}_{3} \mathrm{O}_{4}$ and $\mathrm{Ca}_{3} \mathrm{Co}_{2} \mathrm{O}_{6}$ was observed as indicated by the marked peaks $*$ and ${ }^{+}$, respectively. (003) diffraction peak showing the shift to a lower Bragg's angle. Red line correspond to position of peak in the undoped $\mathrm{Ca}_{3} \mathrm{Co}_{4} \mathrm{O}_{9}$.

XRD patterns of $\mathrm{Ca}_{3-x} \mathrm{~Tb}_{x} \mathrm{Co}_{4} \mathrm{O}_{9} \mathrm{Bi}_{y}$ samples obtained at room temperature conditions from ground powders of sintered pellets are shown in Figure 9-4. The XRD patterns have been plotted with a minor offset with the purpose of a better visualization. The corresponding calculated lattice parameter values for samples with different doping level of the dual doping $\mathrm{Ca}_{3-x} \mathrm{~Tb}_{x} \mathrm{Co}_{4} \mathrm{O}_{9} \mathrm{Bi}_{y}$ are listed in Table 9-3. Previously, it was confirmed that Tb substitution produces a slight shift to a 
higher Bragg's angle of the peaks and also showed a decrease in the lattice parameter values of $a$, $b_{1}, c$, and $\beta$, accompanied by the increase in the lattice parameter $b_{2}$. Figure 9-4 (a) show the dual doping $\mathrm{Ca}_{3-x} \mathrm{~Tb}_{x} \mathrm{Co}_{4} \mathrm{O}_{9} \mathrm{Bi}_{y}$, all XRD characteristic peaks can be indexed as those from the reported $\mathrm{Ca}_{3} \mathrm{Co}_{4} \mathrm{O}_{9}$ phase with monoclinic symmetry ${ }^{[60]}$. Nonetheless, $\mathrm{Ca}_{2.95} \mathrm{~Tb}_{0.05} \mathrm{Co}_{4} \mathrm{O}_{9} \mathrm{Bi}_{0.30}$ samples show a minor amount of $\mathrm{Co}_{3} \mathrm{O}_{4}$ and $\mathrm{Ca}_{3} \mathrm{Co}_{2} \mathrm{O}_{6}$ secondary phases. The dual doping of $\mathrm{Tb}$ stoichiometric substitution and Bi non-stoichiometric addition XRD patterns show a slight shift to a lower Bragg's angle of the peaks for the Bi concentrations up to $y=0.25$. For the higher $\mathrm{Bi}$ concentration there is a shift in the opposite direction, to a higher Bragg's angle. The shift to a lower Bragg's angle indicate the increase in lattice parameter values for the dual doping $\mathrm{Ca}_{2.95} \mathrm{~Tb}_{0.05} \mathrm{Co}_{4} \mathrm{O}_{9} \mathrm{Bi}_{\mathrm{y}}$ ceramics. The calculated lattice parameter values for samples with different dual doping level are listed in Table 9-3. In contrast to the single doped $\mathrm{Ca}_{3-x} \mathrm{~Tb}_{x} \mathrm{Co}_{4} \mathrm{O}_{9}$, with the increase of Bi doping level and keeping $\mathrm{Tb}$ at $x=0.05$, there is an increase in the lattice parameter values $a, b_{1}, c$, and $\beta$, which is accompanied by the decrease in the lattice parameter $b_{2}$.

Table 9-3: Lattice parameters $a, b_{1}, b_{2}, c$, and $\beta$ for the single doped and dual doped $\mathrm{Ca}_{3} \mathrm{Co}_{4} \mathrm{O}_{9}$ samples. $b_{1}$ and $b_{2}$ refer to b-axis lattice parameters in rock-salt layer $\mathrm{Ca}_{2} \mathrm{CoO}_{3}$ and $\mathrm{CoO}_{2}$ layer, respectively.

\begin{tabular}{llllll}
\hline $\mathrm{Ca}_{3-\mathrm{x}} \mathrm{Tb}_{\mathrm{x}} \mathrm{Co}_{4} \mathrm{O}_{9} \mathrm{Bi}_{\mathrm{y}}$ & $\mathrm{a}(\AA)$ & $\mathrm{b}_{1}(\AA)$ & $\mathrm{b}_{2}(\AA)$ & $\mathrm{c}(\AA)$ & $\beta\left(^{\circ}\right)$ \\
\hline $\mathrm{x}=0 ; \mathrm{y}=0$ & 4.859 & 4.562 & 2.747 & 10.849 & 98.711 \\
$x=0.05 ; \mathrm{y}=0$ & 4.858 & 4.552 & 2.749 & 10.847 & 98.697 \\
$\mathrm{x}=0.05 ; \mathrm{y}=0.10$ & 4.878 & 4.573 & 2.744 & 10.879 & 99.052 \\
$\mathrm{x}=0.05 ; \mathrm{y}=0.15$ & 4.880 & 4.571 & 2.743 & 10.876 & 98.955 \\
$\mathrm{x}=0.05 ; \mathrm{y}=0.20$ & 4.880 & 4.566 & 2.743 & 10.880 & 99.038 \\
$\mathrm{x}=0.05 ; \mathrm{y}=0.25$ & 4.915 & 4.562 & 2.738 & 10.926 & 99.725 \\
$\mathrm{x}=0.05 ; \mathrm{y}=0.30$ & 4.848 & 4.548 & 2.752 & 10.862 & 98.377 \\
\hline
\end{tabular}

SEM images of $\mathrm{Ca}_{3-x} \mathrm{~Tb}_{x} \mathrm{Co}_{4} \mathrm{O}_{9} \mathrm{Bi}_{y}$ samples after sintering stage are shown in Figure 9-5 and Fig. 9-6. The size of the crystals was found to decrease with the increase in the Tb doping level in 

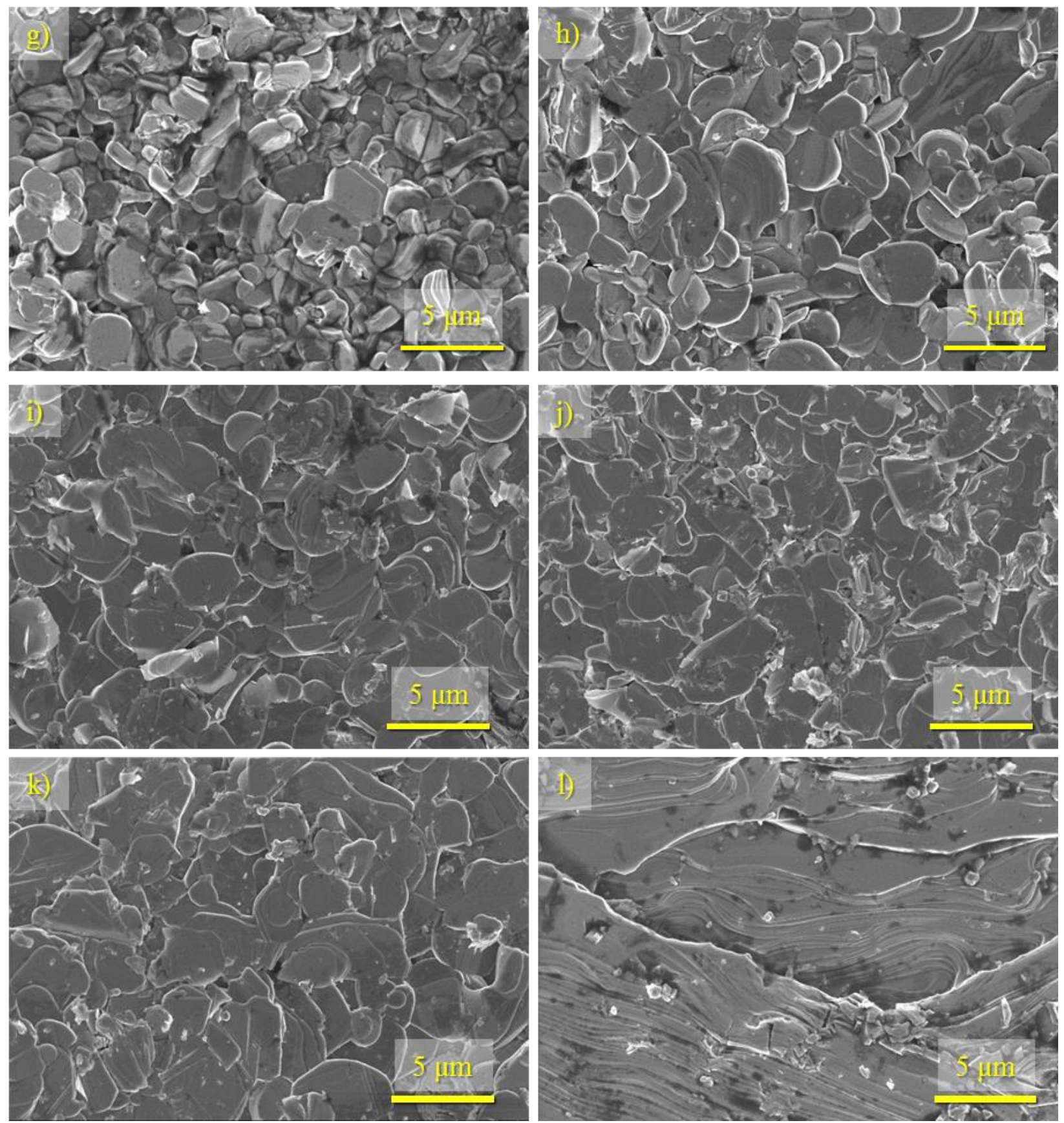

Figure 9-5: SEM plan-view images from the surface of $\mathrm{Ca}_{3-x} \mathrm{~Tb}_{x} \mathrm{Co}_{4} \mathrm{O}_{9} \mathrm{Bi}_{y}$ samples after sintering stage: (a) $\mathrm{Ca}_{3} \mathrm{Co}_{4} \mathrm{O}_{9}$, (b) $\mathrm{Ca}_{2.95} \mathrm{~Tb}_{0.05} \mathrm{Co}_{4} \mathrm{O}_{9} \mathrm{Bi}_{0.10}$, (c) $\mathrm{Ca}_{2.95} \mathrm{~Tb}_{0.05} \mathrm{Co}_{4} \mathrm{O}_{9} \mathrm{Bi}_{0.15}$, (d) $\mathrm{Ca}_{2.95} \mathrm{~Tb}_{0.05} \mathrm{Co}_{4} \mathrm{O}_{9} \mathrm{Bi}_{0.20}$, (e) $\mathrm{Ca}_{2.95} \mathrm{~Tb}_{0.05} \mathrm{Co}_{4} \mathrm{O}_{9} \mathrm{Bi}_{0.25}$, and (f) $\mathrm{Ca}_{2.95} \mathrm{~Tb}_{0.05} \mathrm{Co}_{4} \mathrm{O}_{9} \mathrm{Bi}_{0.30}$

$\mathrm{Ca}_{3-x} \mathrm{~Tb}_{x} \mathrm{Co}_{4} \mathrm{O}_{9}$ samples in previous chapter. Contrariwise, if $\mathrm{Bi}$ is added in top of a $\mathrm{Tb}$ as a second dopant as in $\mathrm{Ca}_{2.95} \mathrm{~Tb}_{0.05} \mathrm{Co}_{4} \mathrm{O}_{9} \mathrm{Bi}_{\mathrm{y}}$ the size of the crystals can be increased gradually with the 

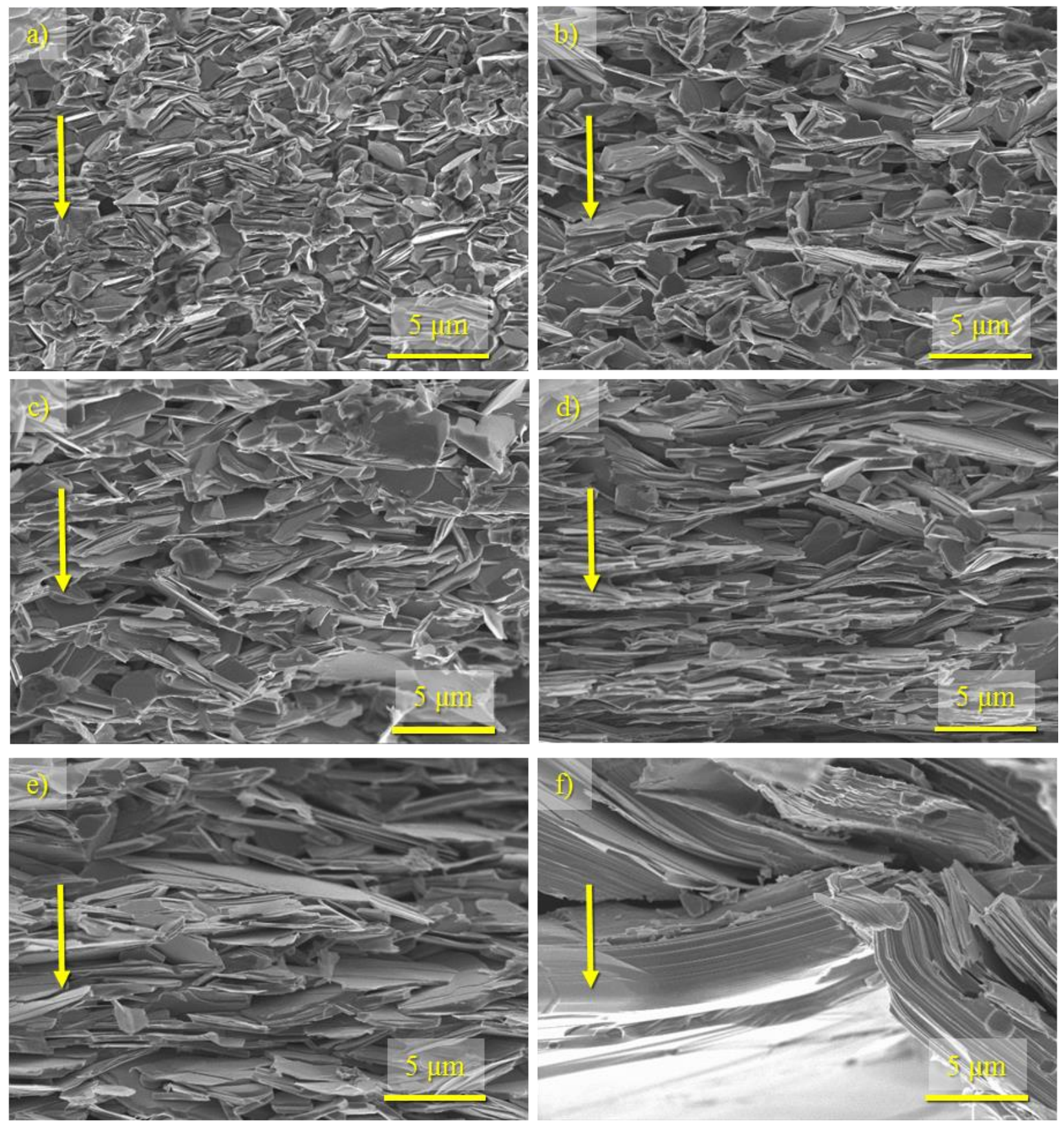

Figure 9-6: SEM cross-sectional images from the fractured surface of $\mathrm{Ca}_{3-x} \mathrm{~Tb}_{x} \mathrm{Co}_{4} \mathrm{O}_{9} \mathrm{Bi}_{y}$ samples

after sintering stage: (a) $\mathrm{Ca}_{3} \mathrm{Co}_{4} \mathrm{O}_{9}$, (b) $\mathrm{Ca}_{2.95} \mathrm{~Tb}_{0.05} \mathrm{CO}_{4} \mathrm{O}_{9} \mathrm{Bi}_{0.10}$, (c) $\mathrm{Ca}_{2.95} \mathrm{~Tb}_{0.05} \mathrm{Co}_{4} \mathrm{O}_{9} \mathrm{Bi}_{0.15}$, (d)

$\mathrm{Ca}_{2.95} \mathrm{~Tb}_{0.05} \mathrm{Co}_{4} \mathrm{O}_{9} \mathrm{Bi}_{0.20}$, (e) $\mathrm{Ca}_{2.95} \mathrm{~Tb}_{0.05} \mathrm{Co}_{4} \mathrm{O}_{9} \mathrm{Bi}_{0.25}$, and $(f) \mathrm{Ca}_{2.95} \mathrm{~Tb}_{0.05} \mathrm{Co}_{4} \mathrm{O}_{9} \mathrm{Bi}_{0.30}$. Arrows represent the pressing direction of the samples.

increase in the Bi doping level as shown in the plan-view SEM images. Figure 9-6 (a)-(f) shows the cross-sectional SEM images taken from the fractured surface of the samples, it can be clearly 
seen that all doping levels present large anisotropy with micron-sized plate-shaped grains having the larger dimension in direction to the $a-b$ plane of the monoclinic $\mathrm{Ca}_{3} \mathrm{Co}_{4} \mathrm{O}_{9}{ }^{[57]}$. The arrow in each cross-sectional SEM image indicates the pressing direction of the pellet. Tb doping deteriorates the crystal texture and produce a more arbitrary crystal grain orientation as shown before. Furthermore, when $\mathrm{Bi}$ is added in top of a $\mathrm{Tb}$ as a second dopant as in $\mathrm{Ca}_{2.95} \mathrm{~Tb}_{0.05} \mathrm{Co}_{4} \mathrm{O}_{9} \mathrm{Bi}_{\mathrm{y}}$ the size of the crystals gradually increases parallel to the $a-b$ plane of the elongated crystal grains, while remained with an equivalent size in the $c$-axes as shown in Figure 9-6 (a)-(e) for samples with Bi up to $y=0.25$. Moreover, it can also be observed a development of the crystal texture that preferentially aligns the crystal grain orientation for the $\mathrm{Ca}_{2.95} \mathrm{~Tb}_{0.05} \mathrm{Co}_{4} \mathrm{O}_{9} \mathrm{Bi}_{y} \leq 0.25$ samples. Most of the crystals have the $c$-axis of the monoclinic structure parallel to the pressed direction of the pellets for $\mathrm{Ca}_{2.95} \mathrm{~Tb}_{0.05} \mathrm{Co}_{4} \mathrm{O}_{9} \mathrm{Bi}_{\mathrm{y} \leq 0.25}$ samples. With the increase in the $\mathrm{Bi}$ doping level to $\mathrm{y}=0.3$, for the $\mathrm{Ca}_{2.95} \mathrm{~Tb}_{0.05} \mathrm{Co}_{4} \mathrm{O}_{9} \mathrm{Bi}_{0.30}$, sample shown in Figure 9-6 (f), the crystal texture diminishes, and the high doping level produce large arbitrary crystal grain not having a preferred orientation.

To better quantify the crystallographic texture orientation degree of $\mathrm{Ca}_{3-\mathrm{x}} \mathrm{Tb}_{\mathrm{x}} \mathrm{Co}_{4} \mathrm{O}_{9} \mathrm{Bi}_{\mathrm{y}}$ samples, the texture fraction (Lotgering factor) of the $\{001\}$ planes was calculated utilizing the intensities of the XRD peaks obtained at room temperature. The Lotgering factor $(L F)$ is defined as $L F=\left(P_{l}-P_{0}\right) /\left(1-P_{0}\right),{ }^{[74]}$ where $P_{l}=\sum I_{0 o l} \sum I_{h k l}$ and $P_{0}=\sum I^{\prime}{ }_{0 o l} \sum I_{h k l}^{\prime}$ denotes the fraction of the summation of the peak intensities for the as sintered pellet sample(textured oriented) and the aftersintering ground sample (randomly oriented), respectively. A fraction close to the unity correspond to the best possible orientation. The integrated densities of each of the corresponding XRD peaks were obtained with the Fullprof software. 
Table 9-4: Lotgering factor for the dual doped $\mathrm{Ca}_{3} \mathrm{Co}_{4} \mathrm{O}_{9}$ samples

\begin{tabular}{lc}
\hline $\mathrm{Ca}_{3-\mathrm{x}} \mathrm{Tb}_{\mathrm{x}} \mathrm{Co}_{4} \mathrm{O}_{9} \mathrm{Bi}_{\mathrm{y}}$ & \multicolumn{1}{c}{$L F$} \\
\hline$x=0 \quad ; y=0$ & 0.678 \\
$x=0.05 ; y=0$ & 0.644 \\
$x=0.50 ; y=0$ & 0.391 \\
$x=0 \quad ; y=0.25$ & 0.968 \\
$x=0.05 ; y=0.25$ & 0.959 \\
\hline
\end{tabular}

To better understand the effect of the dual doping strategy on the nanostructure of the calcium cobaltite ceramics, TEM examination was performed on the dual doped $\mathrm{Ca}_{2.95} \mathrm{~Tb}_{0.05} \mathrm{Co}_{4} \mathrm{O}_{9} \mathrm{Bi}_{0.25}$ (shown in Figure 9-7) samples. TEM images depict that each of the micron-sized crystal grains shown in the SEM images are conformed by a conglomeration of nanolamellas having different $c$ axes. Previously, the TEM analysis showed that the $\mathrm{Ca}_{2.5} \mathrm{~Tb}_{0.5} \mathrm{Co}_{4} \mathrm{O}_{9}$ crystal grains have a large amount of high angle GBs between them. Nonetheless, TEM examination of Figure 9-7 (b) for the $\mathrm{Ca}_{2.95} \mathrm{~Tb}_{0.05} \mathrm{Co}_{4} \mathrm{O}_{9} \mathrm{Bi}_{0.25}$ sample illustrates that is possible to modify this nano size structure to achieve better alignment -and thus, large amount of low angle GBs between them- in the nanolamellas that conform the micron sized crystal grains. Thus, it is plausible to tune the properties of our crystal in the micron and nano size spectrum to achieve a beneficial orientation of the highly anisotropic $\mathrm{Ca}_{3} \mathrm{Co}_{4} \mathrm{O}_{9}$ ceramics. Additionally, TEM results confirmed the singlephase structure of the $\mathrm{Ca}_{2.95} \mathrm{~Tb}_{0.05} \mathrm{Co}_{4} \mathrm{O}_{9} \mathrm{Bi}_{0.25}$ since there is not presence of any secondary phase. Additionally, the high resolution TEM (HRTEM) image in Figure 9-7 (c)-(d) also exposes that there is no secondary phase induced by either of the doping element at the GBs. There is not apparent lattice distortion in either side of the neighboring crystal grains in the GBs region in the high resolution TEM images. Apparently, the Bi segregation does not produce lattice distortion in the neighboring crystals. 


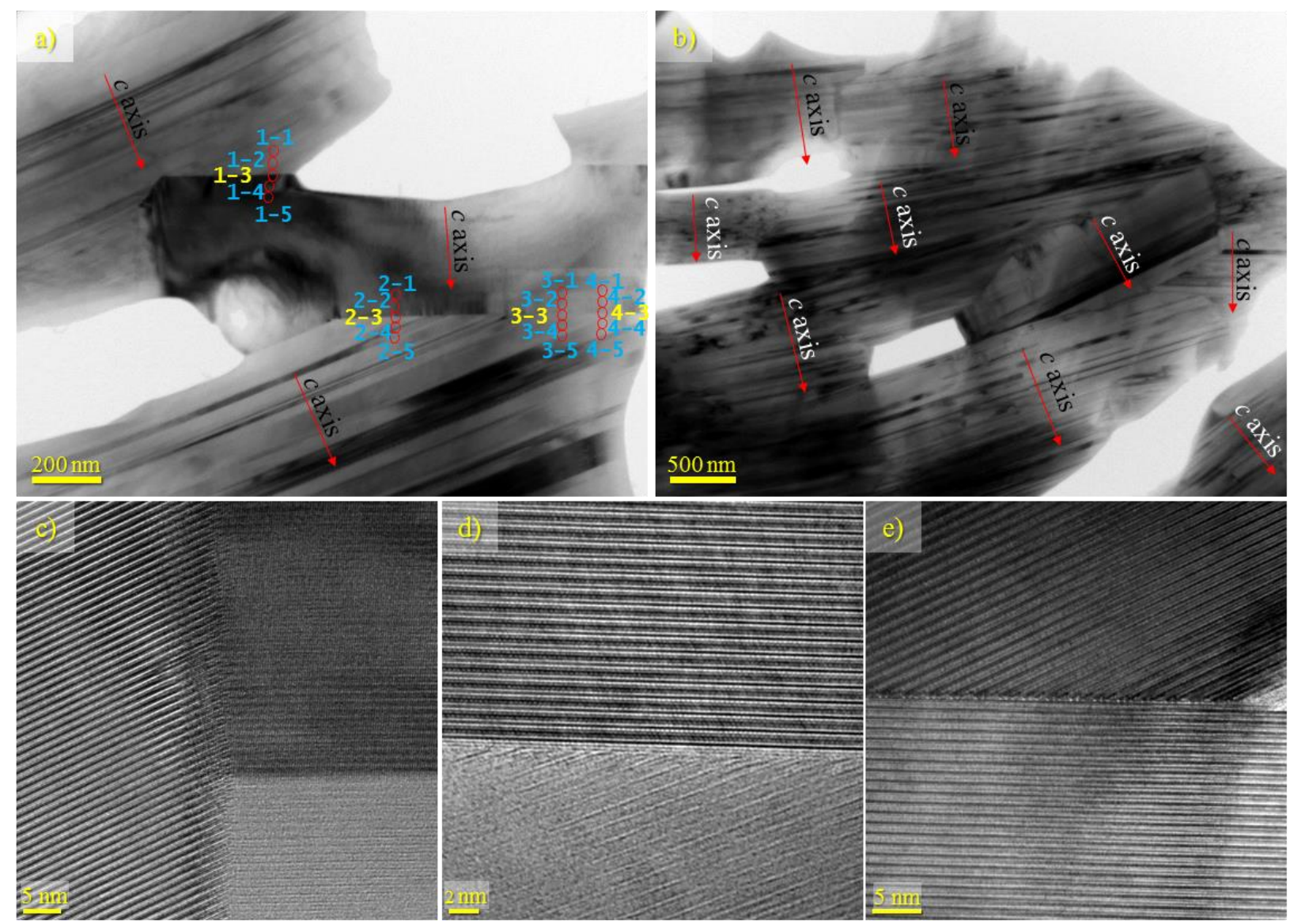

Figure 9-7: TEM images showing the nanostructure of the doped $\mathrm{Ca}_{3} \mathrm{Co}_{4} \mathrm{O}_{9}$ sample. (a) The grain boundary from the crystals with different c-axis orientations. The red circles on the TEM images indicate the electron beam sample areas for the EDS data acquisition. (b) TEM showing adjacent crystals having a preferred crystal orientation in the nanolamellas. (c)-(d) HRTEM showing the low angle GBs between neighboring crystals.

To further explore the chemical changes due to addition of $\mathrm{Tb}$ in conjunction with $\mathrm{Bi}$ as a dual doping, EDS examination was recorded on the GBs and grain interior of $\mathrm{Ca}_{3} \mathrm{Co}_{4} \mathrm{O}_{9}$ crystals to determine the atomic ratio of the different elements. The circle spots in Figure 9-7 (a) correspond to the location of EDS between the adjacent grains. The atomic ratio for each EDS spot is summarized in Table 9-5 for $\mathrm{Ca}_{2.95} \mathrm{~Tb}_{0.05} \mathrm{Co}_{4} \mathrm{O}_{9} \mathrm{Bi}_{0.25}$. 
Table 9-5: Chemistry of the grain interior and grain boundaries of the dual doped $\mathrm{Ca}_{3} \mathrm{CO}_{4} \mathrm{O}_{9}$ sample for different sites labeled in the TEM image in Figure 9-7(a).

\begin{tabular}{llllll}
\hline $\mathrm{At} \%$ & $1-1$ & $1-2$ & $1-3$ & $1-4$ & $1-5$ \\
\hline $\mathrm{Ca}$ & 16.06 & 15.80 & 14.61 & 17.60 & 16.46 \\
$\mathrm{Co}$ & 17.64 & 17.03 & 16.41 & 19.36 & 18.39 \\
$\mathrm{O}$ & 64.27 & 65.09 & 66.69 & 60.99 & 63.22 \\
$\mathrm{~Tb}$ & 0.35 & 0.42 & $\mathbf{0 . 3 2}$ & 0.39 & 0.32 \\
$\mathrm{Bi}$ & 1.69 & 1.66 & $\mathbf{1 . 9 8}$ & 1.67 & 1.60 \\
\hline $\mathrm{At} \%$ & $2-1$ & $2-2$ & $2-3$ & $2-4$ & $2-5$ \\
\hline $\mathrm{Ca}$ & 19.05 & 19.24 & 18.30 & 18.58 & 17.46 \\
$\mathrm{Co}$ & 22.22 & 23.45 & 23.42 & 22.10 & 21.75 \\
$\mathrm{O}$ & 56.20 & 54.80 & 55.02 & 56.84 & 58.55 \\
$\mathrm{~Tb}$ & 0.47 & 0.44 & $\mathbf{0 . 3 9}$ & 0.41 & 0.40 \\
$\mathrm{Bi}$ & 2.06 & 2.08 & $\mathbf{2 . 8 7}$ & 2.06 & 1.84 \\
\hline $\mathrm{At} \%$ & $3-1$ & $3-2$ & $3-3$ & $3-4$ & $3-5$ \\
\hline $\mathrm{Ca}$ & 17.21 & 16.32 & 13.34 & 13.34 & 13.17 \\
$\mathrm{Co}$ & 19.95 & 19.00 & 16.98 & 15.82 & 15.76 \\
$\mathrm{O}$ & 60.73 & 62.64 & 67.52 & 69.24 & 69.38 \\
$\mathrm{~Tb}$ & 0.39 & 0.39 & $\mathbf{0 . 2 4}$ & 0.28 & 0.38 \\
$\mathrm{Bi}$ & 1.72 & 1.65 & $\mathbf{1 . 9 3}$ & 1.32 & 1.31 \\
\hline $\mathrm{At} \%$ & $4-1$ & $4-2$ & $4-3$ & $4-4$ & $4-5$ \\
\hline $\mathrm{Ca}$ & 13.10 & 13.13 & 13.42 & 14.34 & 13.53 \\
$\mathrm{Co}$ & 15.09 & 16.05 & 16.17 & 16.24 & 15.85 \\
$\mathrm{O}$ & 69.97 & 68.99 & 68.34 & 67.52 & 68.91 \\
$\mathrm{~Tb}$ & 0.34 & 0.33 & $\mathbf{0 . 2 5}$ & 0.30 & 0.27 \\
$\mathrm{Bi}$ & 1.50 & 1.50 & $\mathbf{1 . 8 2}$ & 1.60 & 1.45 \\
\hline
\end{tabular}

As shown in Table 9-5 for the dual dopant ceramic, Tb depletion on the GBs is preserved after adding $\mathrm{Bi}$ as a second dopant while also $\mathrm{Tb}$ has presence in the crystal lattice. Additionally, EDS results confirm the presence of $\mathrm{Bi}$ inside the lattice crystal and the simultaneous segregation at the GBs is observed. Hence, EDS results are consistent with the information obtained by XRD analysis that showed the $\mathrm{Ca}_{3} \mathrm{Co}_{4} \mathrm{O}_{9}$ lattice is expanded by the incorporation of $\mathrm{Bi}$ in the dual doping material, even though at the grain boundaries a larger amount of Bi prevails. 


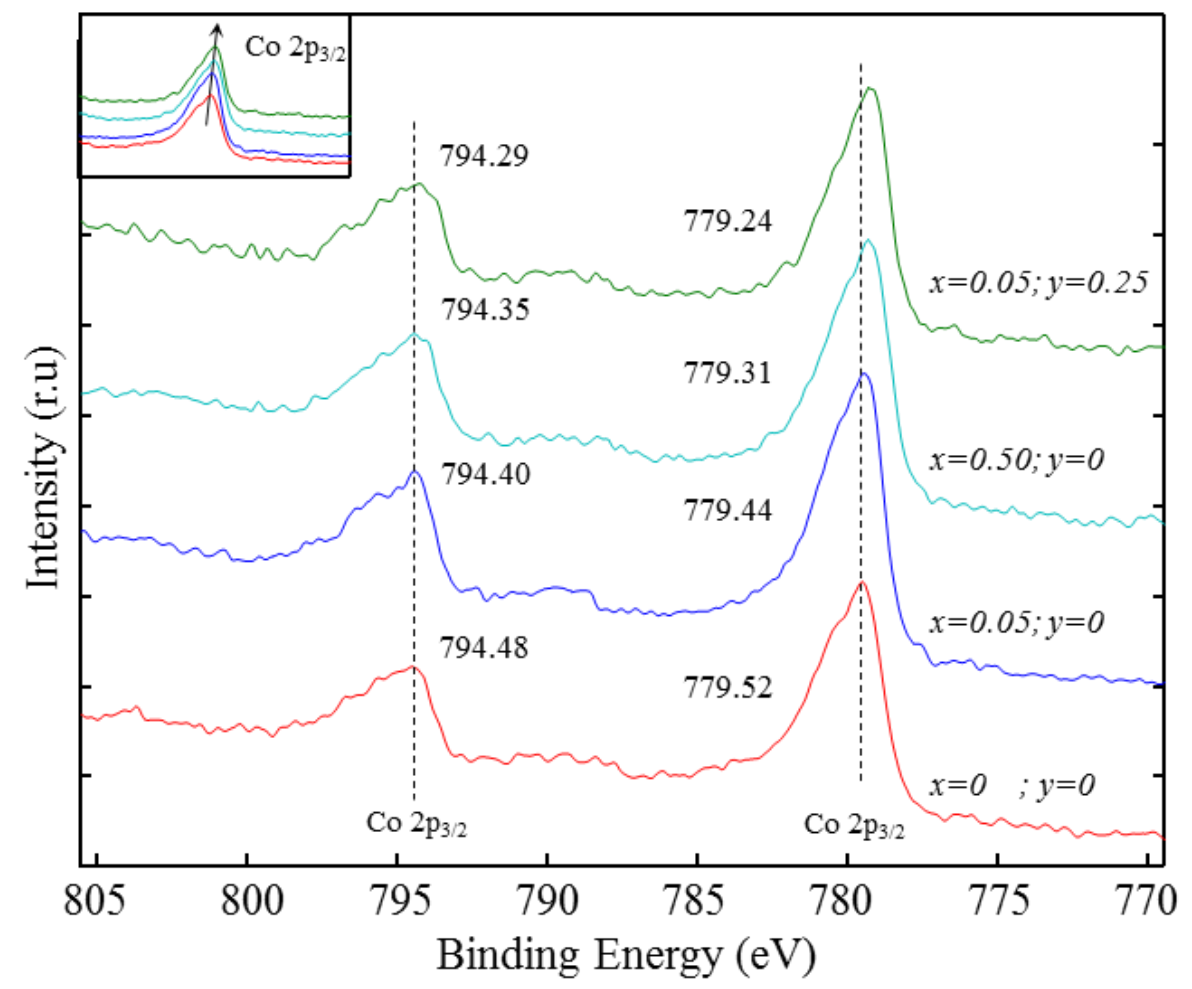

Figure 9-8: $\mathrm{Co} 2 p_{3 / 2}$ and $2 p_{1 / 2}$ peaks from XPS spectrum of $\mathrm{Ca}_{3} \mathrm{Co}_{4} \mathrm{O}_{9}$ samples with dual dopants. Inset figure showing Co 2p $3 / 2$ peaks shifting to a lower binding energy.

Figure 9-8 shows the Co $2 \mathrm{p}_{3 / 2}$ and $2 \mathrm{p}_{1 / 2}$ peaks from XPS spectrum of $\mathrm{Ca}_{3-\mathrm{x}} \mathrm{Tb}_{\mathrm{x}} \mathrm{Co}_{4} \mathrm{O}_{9} \mathrm{Bi}_{\mathrm{y}}$ samples. There is a gradual decrease of the binding energy from both peaks due to the substitution of $\mathrm{Ca}$ by $\mathrm{Tb}$ ions. $\mathrm{Tb}$ ions possesses a higher oxidation state than $\mathrm{Ca}^{2+}$, resulting in a shifting in the Co $2 \mathrm{p}$ peaks. Additionally, with the introduction of $\mathrm{Bi}$ as a second dopant the Co $2 \mathrm{p}$ peaks shift to a slightly lower binding energy in comparison with only using $\mathrm{Tb}$ as a single dopant. Thus, the inset in Figure 9-8 clearly shows Co $2 \mathrm{p}_{3 / 2}$ peaks shifting to a lower binding energy. Bi ions are also present in the crystal lattice, thus, it can be inferred that $\mathrm{Bi}$ substitutes $\mathrm{Ca}^{2+}$ and this produces a reduction of the $\mathrm{Co}^{4+}$ concentration which in turn produces a shift of the Co $2 \mathrm{p}$ XPS peaks to a slightly lower binding energy. 

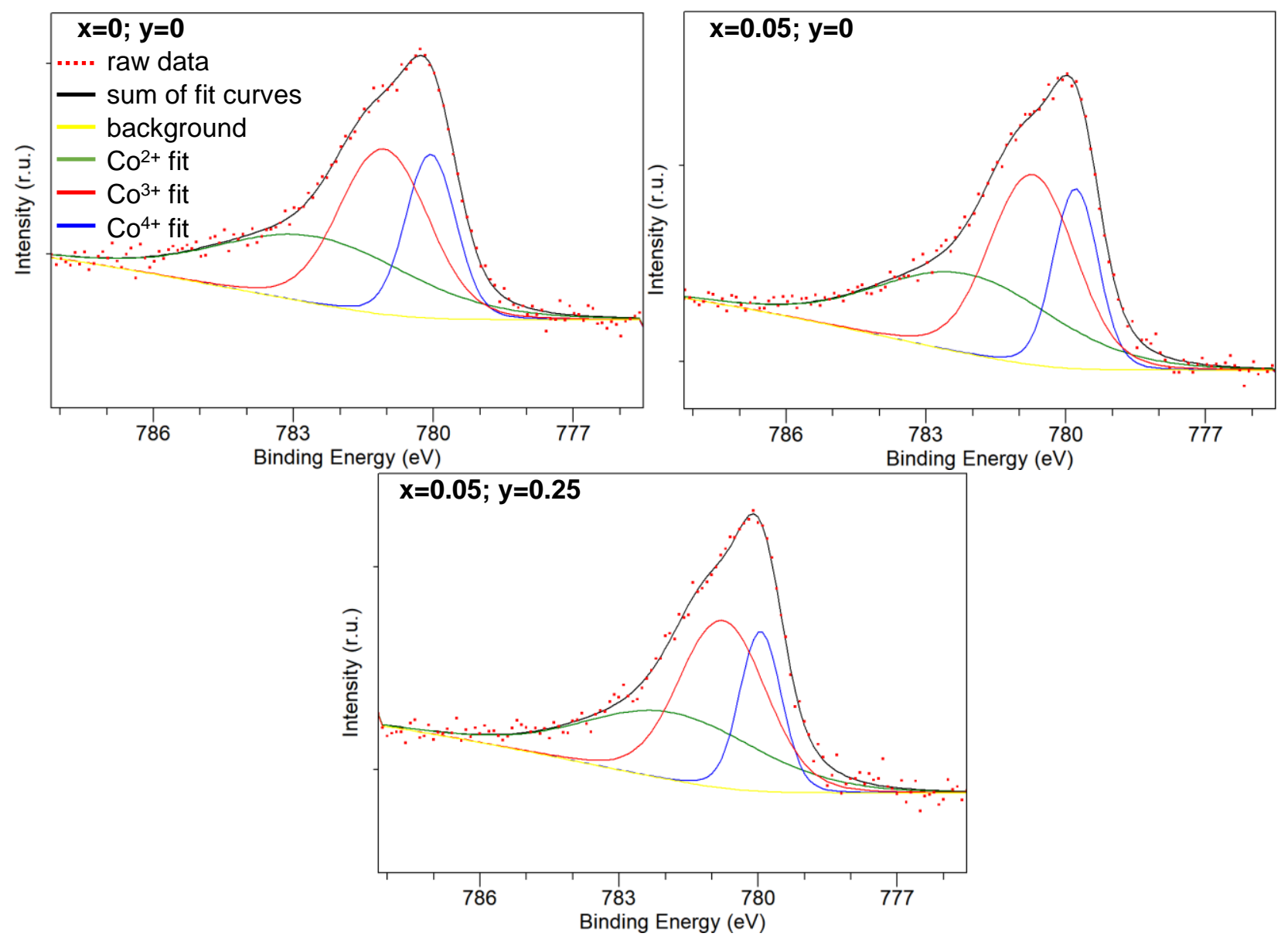

Figure 9-9: Deconvolution of $\mathrm{Co} 2 p_{3 / 2}$ peak from XPS of $\mathrm{Ca}_{3} \mathrm{Co}_{4} \mathrm{O}_{9}$ samples with dual dopants.

The deconvolution of Co $2 \mathrm{p}_{3 / 2}$ peak from XPS spectrums the different oxidation states of Co is presented in Figure 9-9. It is shown that the Co ions have three oxidation states: $\mathrm{Co}^{2+}, \mathrm{Co}^{3+}$ and $\mathrm{Co}^{4+}$ in all samples. Table 9-6 shows the percentage of each oxidation states and the $\mathrm{Co}^{4+} / \mathrm{Co}^{3}$ ratio in the analyzed samples. $\mathrm{Co}^{3+} / \mathrm{Co}^{4+}$ is mostly associated with the $\mathrm{CoO}_{2}$ hexagonal layers and $\mathrm{Co}^{2+} / \mathrm{Co}^{3+}$ is associated with the $\mathrm{Ca}_{2} \mathrm{CoO}_{3}$ rock-salt layers. Therefore, reducing the $\mathrm{Co}^{4+} / \mathrm{Co}^{3}$ ratio in the bulk material could lead to an improved the thermopower.

Table 9-6: Ratios of $\mathrm{Co}^{4+}, \mathrm{Co}^{3+}$ and $\mathrm{Co}^{2+}$ from the XPS deconvolution of the Co $2 p_{3 / 2}$ peak

\begin{tabular}{lcccc}
\hline $\mathrm{Ca}_{3-\mathrm{x}} \mathrm{Tb}_{\mathrm{x}} \mathrm{Co}_{4} \mathrm{O}_{9} \mathrm{Bi}_{\mathrm{y}}$ & $\mathrm{Co}^{4+}(\%)$ & $\mathrm{Co}^{3+}(\%)$ & $\mathrm{Co}^{2+}(\%)$ & $\mathrm{Co}^{4+} / \mathrm{Co}^{3+}(\%)$ \\
\hline$x=0 \quad ; y=0$ & 23.06 & 35.30 & 41.64 & 0.653 \\
$x=0.05 ; y=0$ & 20.99 & 43.01 & 36.00 & 0.488 \\
$x=0.05 ; y=0.25$ & 20.32 & 44.76 & 34.92 & 0.453 \\
\hline
\end{tabular}




\section{5 Discussion}

Doping of $\mathrm{Tb}$ into $\mathrm{Ca}_{3} \mathrm{Co}_{4} \mathrm{O}_{9}$ ceramics is incorporated in the crystal lattice, as has been demonstrated in previous reports ${ }^{[50]}$, and also confirmed in our research. Tb triggered the reduction of the lattice by replacing the $\mathrm{Ca}^{2+}$ sites by smaller $\mathrm{Tb}$ ions. In contrast, the results for the dual doping material, the $\mathrm{Bi}^{3+}$ ionic radius is $117 \mathrm{pm}$, which is slightly bigger than the host ion $\mathrm{Ca}^{2+}$ is $114 \mathrm{pm}$. Therefore, the difference in the ionic radius produces a slight shift to a lower Bragg's angle of the XRD peaks for the dual doped materials. For the highest Bi concentration there is a shift in the opposite direction, to a higher Bragg's angle. Thus, in contrast to the single doped Tb material, $\mathrm{Bi}$ ionic radius is bigger than the $\mathrm{Ca}_{3} \mathrm{Co}_{4} \mathrm{O}_{9}$ hosting ions, hence, the non-stoichiometric addition of the bigger $\mathrm{Bi}$ atom is the cause in the increase of the crystal lattice. Thus, suggesting the non-stoichiometric addition of $\mathrm{Bi}$ atoms in the dual doping material are also being incorporated in place of Ca lattice sites, supporting the information from previous research ${ }^{[43,48]}$. Actually, previous research in $\mathrm{Bi}$ substitution ${ }^{[43]}$ demonstrated that the segregation of $\mathrm{Bi}$ at GBs. Whereas, a report on $\mathrm{Bi}$ and $\mathrm{Ba}$ dual doping ${ }^{[48]}$ showed that $\mathrm{Bi}$ is depleted at the $\mathrm{GBs}$, likely due to the bigger ion $\mathrm{Ba}$ pushing away $\mathrm{Bi}$ in the GBs. In the present dual doping material, $\mathrm{Bi}$ is the bigger of the atoms and is segregated at a higher rate at the GBs since the competing dopant $\mathrm{Tb}$ is smaller than $\mathrm{Bi}$ and the host ions in $\mathrm{Ca}_{3} \mathrm{Co}_{4} \mathrm{O}_{9}$. In this dual doping material, $\mathrm{Tb}$ facilitates the $\mathrm{Bi}$ GBs segregation. Thus, we provide further evidence of the $\mathrm{Bi}^{3+}$ segregation at the GBs using a nonstoichiometric addition of the $\mathrm{Bi}$ dopant in a dual doping setting. The GBs structure on the $\mathrm{Ca}_{3} \mathrm{Co}_{4} \mathrm{O}_{9}$ ceramics could be modified by the inclusion of an ion-size mismatch between the dopant and host ions ${ }^{[41,43,48,53]}$.

$\mathrm{Ca}_{3} \mathrm{Co}_{4} \mathrm{O}_{9}$ is comprised of a monoclinic structure with two misfit layered subsystems aligned in the $c$-axis, the $\mathrm{CoO}_{2}$ level functions as an electrical conductive layer and the $\mathrm{Ca}_{2} \mathrm{CoO}_{3}$ level 
owns a characteristic electrical insulating. Each individual layer in $\mathrm{Ca}_{3} \mathrm{Co}_{4} \mathrm{O}_{9}$ is capable to contribute effectively in the electronic and ionic transport, according to recent reports ${ }^{[61]}$. The large grade of anisotropy on the crystal has a large effect on the thermoelectric properties of the ceramic. In fact, the single crystal $\rho$ in the $a-b$ basal plane of the monoclinic unit cell is significantly lower than along the $c$-axes direction ${ }^{[20]}$. In polycrystalline $\mathrm{Ca}_{3} \mathrm{Co}_{4} \mathrm{O}_{9}$, a superior crystal texture results in better orientation of the lamellas along the $c$-axes, but also important is to improve the nanolamella orientation within the crystal to significantly improve on the electrical properties of the material.

As SEM and TEM showed previously, Tb reduces considerably the size of the $\mathrm{Ca}_{3} \mathrm{Co}_{4} \mathrm{O}_{9}$ ceramics, but particularly generates a worst random orientation of the crystal grains in both the micron sized crystals and in the nanollamelas conforming such crystals. This yields a larger $\rho$ in the $\mathrm{Ca}_{3-\mathrm{x}} \mathrm{Tb}_{\mathrm{x}} \mathrm{Co}_{4} \mathrm{O}_{9}$ ceramics and a decrease in the PF performance. Bi addition as a second dopant in $\mathrm{Ca}_{2.95} \mathrm{~Tb}_{0.05} \mathrm{Co}_{4} \mathrm{O}_{9} \mathrm{Bi}_{y}$ improves the crystal texture with most of crystal aligned with the $a-b$ plane perpendicular to the pressing direction as illustrated in the SEM images and additionally promotes the grain size growth. More notably, nanolamellas also exhibited a better alignment in the TEM images. Bi segregation at GBs has been reported to benefit the electrical transport properties as well as the crystal texture of the $\mathrm{Ca}_{3} \mathrm{Co}_{4} \mathrm{O}_{9}$ ceramics ${ }^{[43,48]}$. The improved crystal texture, stimulated crystal alignment, and the grain size development are linked with the increase in carrier mobility in the polycrystalline materials ${ }^{[62,63]}$. The electrical conductivity $\sigma$ satisfies $\sigma=n e \mu$, where $\mu$ is the carrier mobility, $e$ is the elementary positive charge constant, and $n$ is the number density of electrons, thus, the reduction of $\rho$ in ceramics is caused from an improved of carrier mobility, since the improvement of $S$ is correlated to the reduction of carrier concentration. With the utilization of stoichiometric substitution of $\mathrm{Tb}$ and non-stoichiometric addition of $\mathrm{Bi}$ into $\mathrm{Ca}_{3} \mathrm{Co}_{4} \mathrm{O}_{9}$, it was 
possible to enhance the electrical properties by the improved carrier mobility due to the promoted crystal texture, enlarged grain growth, and the upgraded anisotropy orientation. Furthermore, it has been reported that ion diffusion occurs due to the oxygen vacancies and disorder between the different $\mathrm{Co}-\mathrm{O}$ layers and $\mathrm{Ca}-\mathrm{O}$ layers in the rock salt layers ${ }^{[20,64,65]}$ and that ion transport could be increased by doping on the calcium site ${ }^{[65,66]}$. Thus, the substitution of $\mathrm{Tb}$ and $\mathrm{Bi}$ for $\mathrm{Ca}$ in the rock salt layer decreases the concentration of $\mathrm{Co}^{4+}$ and thereby the hole carriers in the $\mathrm{Co}^{3+}$ matrix -confirmed by a shift in XPS to a lower binding energy for the co-doping material-which facilitate oxygen ionic pathways, and thus, probably lead to a higher ionic conductivity, and overall a higher electrical PF. However, the electrical properties are completely diminished by the deteriorated texture and arbitrarily oriented crystals in $\mathrm{Ca}_{2.95} \mathrm{~Tb}_{0.05} \mathrm{Co}_{4} \mathrm{O}_{9} \mathrm{Bi}_{0.30}$.

The substantial increase in the $\mathrm{S}$ values from the dual doped samples infers a decrease in the carrier concentration of the $\mathrm{Ca}_{3} \mathrm{Co}_{4} \mathrm{O}_{9}$ ceramics. At high temperature $(T \rightarrow \infty)$ the Seebeck coefficient of the $\mathrm{Ca}_{3} \mathrm{Co}_{4} \mathrm{O}_{9}$ can be expressed with Heike's formula as ${ }^{[67]}$ :

$$
S_{(T \rightarrow \infty)}=-\frac{k_{B}}{e} \ln \left(\frac{g_{3}}{g_{4}} \frac{x}{1-x}\right)
$$

where $\mathrm{g}_{3}$ and $\mathrm{g}_{4}$ is the number of configuration of the $\mathrm{Co}^{3+}$ and $\mathrm{Co}^{4+}$ ions, respectively, and $\mathrm{x}$ is the fraction of $\mathrm{Co}^{4+}$ in the $\mathrm{CoO}_{2}$ layer. $k_{B}$ and $e$ are the Boltzmann constant and the electronic charge, respectively. It can be expected that the magnitude of $S$ increase with a reduction of $\mathrm{Co}^{4+}$ concentration. Additionally it has been reported that the segregation at the GBs acts as a barrier layer mechanism in bulk semiconductor originated by a band offset among the grain boundary and the grain interior ${ }^{[68]}$. For the single dopant of $\mathrm{Tb}$, there is a gradual shift to a lower binding energy for the Co $2 p_{3 / 2}$ and $2 p_{1 / 2}$ peaks from XPS spectrum due to the substitution of $\mathrm{Ca}^{2+}$ by superior valance $\mathrm{Tb}$ ions. Moreover, with the introduction of $\mathrm{Bi}$ as a second dopant the Co $2 \mathrm{p}$ peaks shift to a slightly lower binding energy. Thus, Bi shifts to a further lower binding energy the Co $2 p$ 
peaks. $\mathrm{Bi}$ was confirmed to substitute the $\mathrm{Ca}^{2+}$ sites in the $\mathrm{Ca}_{2.95} \mathrm{~Tb}_{0.05} \mathrm{Co}_{4} \mathrm{O}_{9} \mathrm{Bi}_{y}$ material and this also yields a reduction of the $\mathrm{Co}^{4+}$ concentration which in turn enhances $S$. Furthermore, it has been reported that the ratio between $\mathrm{Co}^{3+} / \mathrm{Co}^{4+}$ is also important for the enhancement of thermopower ${ }^{[15]}$. The segregation of $\mathrm{Bi}$ at the GBs acts as an obstructive layer to block the carrier transport through adjacent crystals also works to filter carrier with low energy ${ }^{[69]}$, for that reason, carrier motion across the grain boundary only take place to carriers with high energy, thus, contributed to reducing the carrier concentration. The selective filter of low-energy carriers drastically improved $S$ in the polycrystalline $\mathrm{Ca}_{3} \mathrm{Co}_{4} \mathrm{O}_{9}$. There is an evident enhancement of $S$ across the whole temperature regime analyzed for the dual doping material.

The total $\kappa$ in $\mathrm{Ca}_{3} \mathrm{Co}_{4} \mathrm{O}_{9}$ is a cumulative effect of the electronic thermal conductivity $\left(\kappa_{e}\right)$ and the lattice thermal conductivity $\left(\kappa_{i}\right)$. Nevertheless, $\mathrm{Ca}_{3} \mathrm{Co}_{4} \mathrm{O}_{9}$ ceramics predominant component is the contribution of $\kappa_{i}$. Improvements in the electronic transport properties, leads to an increase in the $\kappa_{e}$ component. Still, $\kappa_{e}$ possess a less significant impact on the overall $\kappa$. Tb replaces the host ion $\mathrm{Ca}$ in $\mathrm{Ca}_{3} \mathrm{Co}_{4} \mathrm{O}_{9}$, but it is much heavier in atomic mass. Thus, introducing a heavier atom in the lattice site unquestionably intensifies the phonon scattering events. The scattering is attributed to the difference in atomic mass in the ions. Heavier ions vibrate easily from their neighboring light ions which cause local vibration that result in a shortened phonon mean free path ${ }^{[70]}$. In the $\mathrm{Ca}_{3-}$ ${ }_{x} \mathrm{~Tb}_{\mathrm{x}} \mathrm{Co}_{4} \mathrm{O}_{9} \mathrm{Bi}_{\mathrm{y}}$ ceramics, the non-stoichiometric addition of $\mathrm{Bi}$ also plays an important part in the thermal properties of the samples. The increasing Bi doping levels up to $y \leq 0.25$ reduces the $\kappa_{i}$. The decrease of the $\kappa_{i}$ is related to the existence of $\mathrm{Bi}$ at the $\mathrm{Ca}_{3} \mathrm{Co}_{4} \mathrm{O}_{9}$ crystal lattices since $\mathrm{Bi}$ holds a much heavier atomic mass. For that reason, the heavier $\mathrm{Bi}$ atom presence in the lattice increases the phonon scattering events, as is the case in $\mathrm{Bi}$ doping to $\mathrm{Ca}_{3} \mathrm{Co}_{4} \mathrm{O}_{9}{ }^{[43]}$. Additional contribution to a lower $\kappa_{i}$ is possible to the $\mathrm{Bi}$ segregation at the GBs, this intensifies the phonon scattering 

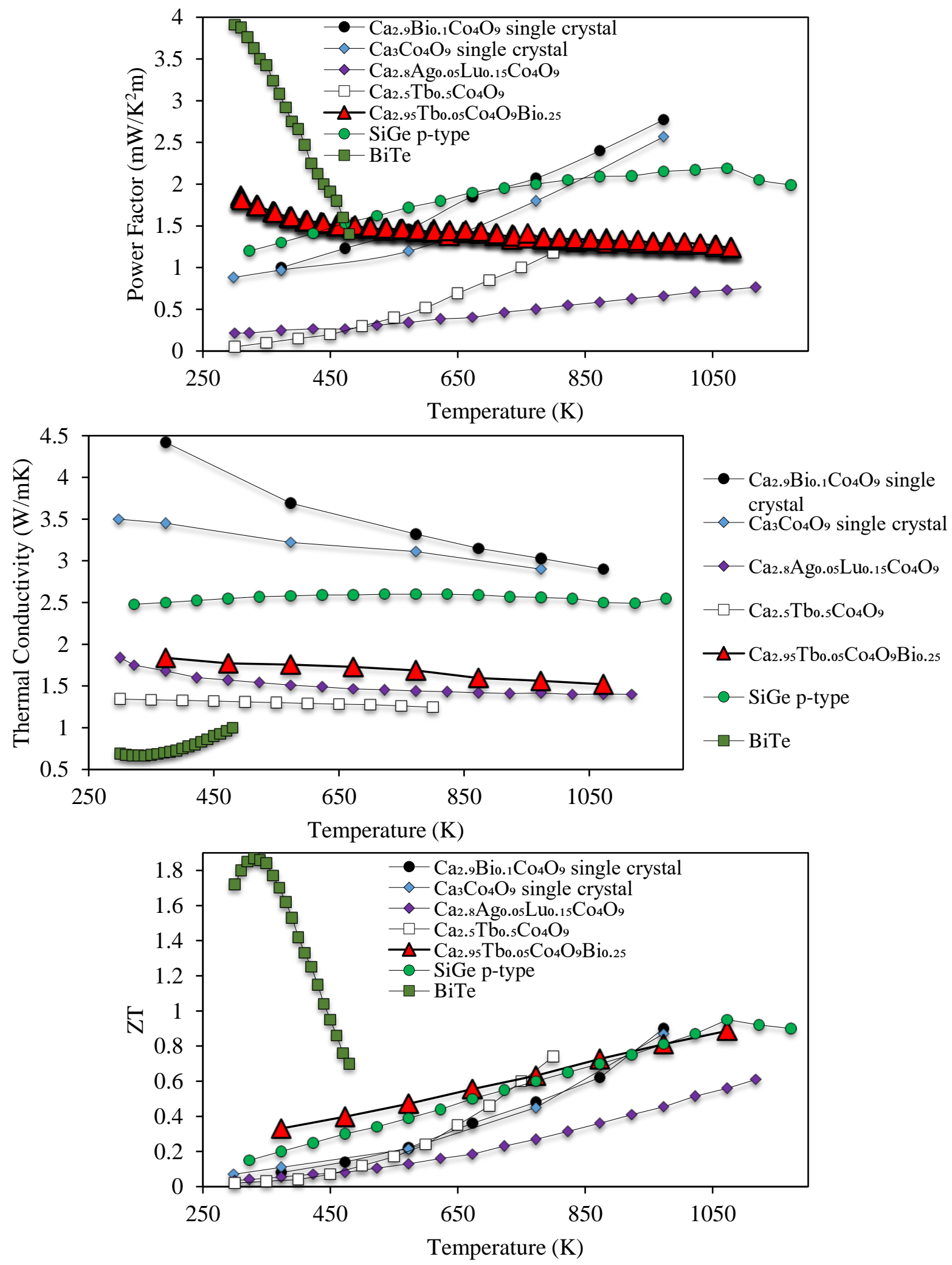

Figure 9-10: Temperature dependence of thermoelectric properties for different state-of-the-art p-type materials: (a) $\mathrm{S}^{2} / \rho-\mathrm{T}$, (b) $\kappa-\mathrm{T}$, and (c) ZT. 
events at the GBs ${ }^{[67,71]}$. Notwithstanding, with the increase in Bi doping level, the electronic transport properties are improved, and thus the contribution of $\kappa_{e}$ increases. For the best performing $\mathrm{Ca}_{2.95} \mathrm{~Tb}_{0.05} \mathrm{Co}_{4} \mathrm{O}_{9} \mathrm{Bi}_{0.25}$ material, the increase of $\kappa_{e}$ becomes noticeable, but notably the overall $\kappa$ remains lower than the pristine $\mathrm{Ca}_{3} \mathrm{Co}_{4} \mathrm{O}_{9}$. As Figure 9-10 (b) shows, $\mathrm{Ca}_{2.95} \mathrm{~Tb}_{0.05} \mathrm{Co}_{4} \mathrm{O}_{9} \mathrm{Bi}_{0.25} \mathrm{~K}$ is lower than the $\mathrm{Ca}_{3} \mathrm{Co}_{4} \mathrm{O}_{9}$ single crystals ${ }^{[20,24]}$ since it holds a better chance to scatter phonon and reduce the predominant contribution of $\kappa$. However there are conventional p-type materials with lower reported $\kappa$, such as $\mathrm{SiGe}^{[7]}$ and $\mathrm{BiTe}^{[6]}$.

The increase of $S$ coupled with an almost unchanged $\rho$ values the reduction of $\kappa$ in $\mathrm{Ca}_{2.95} \mathrm{~Tb}_{0.05} \mathrm{Co}_{4} \mathrm{O}_{9}$ materials produced a subtle increase in the $Z T$ up to 0.38 at $1073 \mathrm{~K}$ compared to the value of 0.35 for pristine $\mathrm{Ca}_{3} \mathrm{Co}_{4} \mathrm{O}_{9}$. Nonetheless, this subtle improvement served as an higher starting point to further increase the $Z T$ with the dual doping approach. The dual nonstoichiometric addition of $\mathrm{Bi}$ and stoichiometric substitution of $\mathrm{Tb}$ reduces the thermal conductivity $\kappa$ in comparison to the pristine $\mathrm{Ca}_{3} \mathrm{Co}_{4} \mathrm{O}_{9}$ and is also complemented by the pronounced simultaneous reduction of $\rho$ and improved $S$. Thus, the $Z T$ is exceptionally enhanced for the dual doping approach, resulting in a ZT of $\sim 0.89$ at $1073 \mathrm{~K}$. This magnitude represent the thus far the highest $Z T$ and the first time a polycrystalline calcium cobaltite has exceeded the performance of the single crystal at high temperature, as shown in Figure 9-10 (c). Additionally, the peak ZT of 0.89 is significantly higher than the best reported ZT of 0.61 from $\mathrm{Ca}_{2.8} \mathrm{Ag}_{0.05} \mathrm{Lu}_{0.15} \mathrm{Co}_{4} \mathrm{O}_{9}{ }^{[34]}$ synthesized using specialized Spark Plasma Sintering. The ZT value of $\mathrm{Ca}_{2.95} \mathrm{~Tb}_{0.05} \mathrm{Co}_{4} \mathrm{O}_{9} \mathrm{Bi}_{0.25}$ even surpasses the widely used $\mathrm{SiGe}^{[7]}$ performance at temperatures lower than $973 \mathrm{~K}$ as can be seen in Figure 9-10 (c). . Most of all, $\mathrm{Ca}_{3} \mathrm{Co}_{4} \mathrm{O}_{9-8}$ ceramics are having just 5$10 \%$ of the cost of SiGe, ${ }^{[72]}$ and will be performing directly in air. 
Although, there has been a key improvement in the performance of $\mathrm{Ca}_{3} \mathrm{Co}_{4} \mathrm{O}_{9}$ ceramics. Thus far the low electrical PF -shown in Figure 9-10 (a)- is still a big challenge to further improve the ZT for TE oxide materials. Some TE materials own a superior ZT performance, but their commercialization is objectionable due to their restrictions such as poor thermal stability at high temperature in air, low abundance, high cost of the comprising elements, and toxicity ${ }^{[9]}$. The improvement on the ZT performance of the proposed material with the inherent benefits of the oxide make $\mathrm{Ca}_{3} \mathrm{Co}_{4} \mathrm{O}_{9}$ a competitive candidate to remain relevant with conventional and emerging p-type thermoelectric materials.

Most notably, the designed chemistry is tunable in term of dopant species and doping level. As an example, we tested the sample of $\mathrm{Ca}_{2.94} \mathrm{Pr}_{0.03} \mathrm{~Tb}_{0.03} \mathrm{Co}_{4} \mathrm{O}_{9} \mathrm{Bi}_{0.23}$ by introducing the third element Pr and slightly lower the Bi doping level. The electrical power factor was lowered slightly, however, the thermal conductivity was also lowered due to the increased entropy. Eventually, the sample $\mathrm{Ca}_{2.94} \mathrm{Pr}_{0.03} \mathrm{~Tb}_{0.03} \mathrm{Co}_{4} \mathrm{O}_{9} \mathrm{Bi}_{0.23}$ is with comparable $\mathrm{ZT}$ to that of $\mathrm{Ca}_{2.95} \mathrm{~Tb}_{0.05} \mathrm{Co}_{4} \mathrm{O}_{9-\delta} \mathrm{Bi}_{0.25}$ as shown in Figure 9-11.

Lastly, it is important to remark that the methods utilized in the present work for producing the high-performance oxide ceramics is conventional and low cost, can be readily scale up for massive production to allow the oxide to play a central role in the TE technology. Additionally, the sample with designed chemistry of $\mathrm{Ca}_{2.95} \mathrm{~Tb}_{0.05} \mathrm{Bi}_{0.25} \mathrm{Co}_{4} \mathrm{O}_{9-\delta}$ is with high plateau of $\mathrm{ZT} 0.33$ at $373 \mathrm{~K}$ and peak ZT of 0.89 at $1073 \mathrm{~K}$. Such high plateau leads to the high average $\mathrm{ZT}^{[73]}$, which is very much desired for high performance TE materials. 


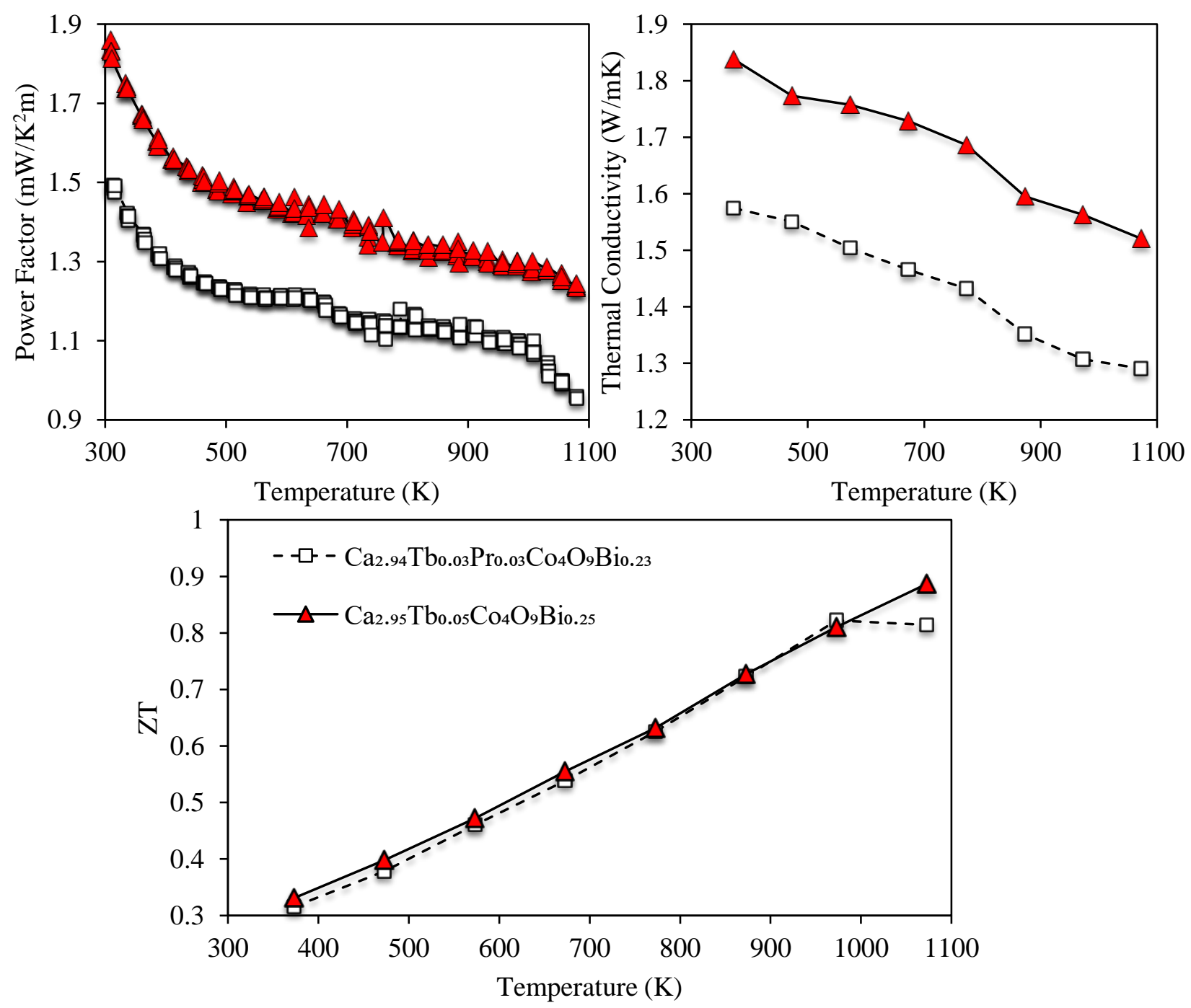

Figure 9-11: Temperature dependence of thermoelectric properties for different dopant species and doping level of high-performance $\mathrm{Ca}_{3} \mathrm{Co}_{4} \mathrm{O}_{9}$ ceramics.

\section{6 Conclusion}

The current work has explored a methodical dual doping approach to improve the TE performance of $\mathrm{Ca}_{3} \mathrm{Co}_{4} \mathrm{O}_{9}$ ceramics. First, $\mathrm{Tb}$ stoichiometric substitution for $\mathrm{Ca}$ was previously analyzed to evaluate the effect on TE properties and microstructure of the material. Tb doping achieved a $10 \%$ performance increase to a $\mathrm{ZT}$ of 0.38 . After analyzing the effects of $\mathrm{Tb}$ and selecting the optimal concentration, Bi was added to further promote the TE properties to achieve 
the highest ZT of $\sim 0.9$ for $\mathrm{Ca}_{2.95} \mathrm{~Tb}_{0.05} \mathrm{Co}_{4} \mathrm{O}_{9} \mathrm{Bi}_{0.25}$ ceramics. This approach led to a set of perceptible effects for each element to modify the structure and TE properties for a specific purpose, such as a 4.5 times improvement on the electrical PF and the beneficial $12 \%$ reduction on the thermal transport properties. TEM results showed evidence of $\mathrm{Tb}$ at the crystal lattice and depletion at the GBs, whereas $\mathrm{Bi}$ addition is present in the crystal lattice but with segregation at the GB due to the larger $\mathrm{Bi}$ atom. Lattice parameter increase with $\mathrm{Bi}$ incorporation, thus, likely substitution of $\mathrm{Bi}$ at $\mathrm{Ca}^{2+}$ sites exist. The segregation of $\mathrm{Bi}$ is attributed to the dissimilarity in its ionic radius in comparison with the $\mathrm{Ca}_{3} \mathrm{Co}_{4} \mathrm{O}_{9}$ host ions. SEM images showed a developed crystal texture and improvement crystal orientation of the micron sized with the dual doping approach. Notably, TEM showed the possibility to further improve the crystal orientation in the nano size spectrum. Employing the dual doping stoichiometric and non-stoichiometric of $\mathrm{Tb}$ and $\mathrm{Bi}$, respectively, the $\mathrm{Ca}_{3} \mathrm{Co}_{4} \mathrm{O}_{9}$ properties are manipulated to preserve advantages of both dopants. This resulted in $S$ values with $15 \%$ to $10 \%$ increasement from $320 \mathrm{~K}$ and $1073 \mathrm{~K}$, (due to reduced carrier concentration from carrier filtering at GBs) and a $70 \%$ to $40 \%$ reduction in the $\rho$ values from $320 \mathrm{~K}$ and $1073 \mathrm{~K}$ (due to enhanced carrier mobility). Carrier concentration was reduced due to doping of higher oxidation state in place of the $\mathrm{Ca}^{2+}$ sites in the system. Notably, the inclusion of the heavier atoms in the lattice sites of $\mathrm{Ca}_{3} \mathrm{Co}_{4} \mathrm{O}_{9}$ ceramics increases the phonon scattering which in turn lead to a $12 \%$ reduction in $\kappa$ of $\mathrm{Ca}_{3} \mathrm{Co}_{4} \mathrm{O}_{9}$ ceramic. Overall, the optimal concentration $\mathrm{Ca}_{2.95} \mathrm{~Tb}_{0.05} \mathrm{Co}_{4} \mathrm{O}_{9} \mathrm{Bi}_{0.25}$ simultaneously decreases $\rho$ to lowest value $\sim 13 \mu \Omega \mathrm{m}$ and a low $\kappa$ value $\sim 1.48 \mathrm{~W} / \mathrm{mK}$ while also increasing the Seebeck coefficient to a value of $\sim 210 \mu \mathrm{VK}^{-1}$ in order to achieve a better $\mathrm{TE}$ performance. The outstanding $\mathrm{ZT} \sim 0.9$ at $1073 \mathrm{~K}$ for the reported polycrystalline material represents a value 2.6 times larger than the pristine material and might provide a suitable option to develop high-temperature TE devices. 


\section{References}

1. Chu, S. and A. Majumdar, Opportunities and challenges for a sustainable energy future. Nature, 2012. 488: p. 294.

2. Dusastre, V. and L. Martiradonna, Materials for sustainable energy. Nature Materials, 2016. 16: p. 15.

3. Fergus, J.W., Oxide materials for high temperature thermoelectric energy conversion. Journal of the European Ceramic Society, 2012. 32(3): p. 525-540.

4. Koumoto, K., et al., Thermoelectric Ceramics for Energy Harvesting. Journal of the American Ceramic Society, 2013. 96(1): p. 1-23.

5. Snyder, G.J. and E.S. Toberer, Complex thermoelectric materials. Nature Materials, 2008. 7: p. 105.

6. Kim, S.I., et al., Dense dislocation arrays embedded in grain boundaries for highperformance bulk thermoelectrics. Science, 2015. 348(6230): p. 109.

7. Joshi, G., et al., Enhanced Thermoelectric Figure-of-Merit in Nanostructured p-type Silicon Germanium Bulk Alloys. Nano Letters, 2008. 8(12): p. 4670-4674.

8. Nesbitt, J.A., Rate of Sublimation of $\mathrm{Yb}_{14} \mathrm{MnSb}_{11}$, a Thermoelectric Material for Space Power Applications. Journal of Electronic Materials, 2014. 43(9): p. 3128-3137.

9. Ohtaki, M., Recent aspects of oxide thermoelectric materials for power generation from mid-to-high temperature heat source. Journal of the Ceramic Society of Japan, 2011. 119(1395): p. 770-775.

10. Terasaki, I., Y. Sasago, and K. Uchinokura, Large thermoelectric power in $\mathrm{NaCo}_{2} \mathrm{O}_{4}$ single crystals. Physical Review B, 1997. 56(20): p. R12685-R12687.

11. Fouassier, C., et al., Sur de nouveaux bronzes oxygénés de formule $\mathrm{Na}_{\chi} \mathrm{CoO}_{2}(\chi 1)$. Le système cobalt-oxygène-sodium. Journal of Solid State Chemistry, 1973. 6(4): p. 532-537.

12. Takada, K., et al., Superconductivity in two-dimensional $\mathrm{CoO}_{2}$ layers. Nature, 2003. 422: p. 53.

13. Mizushima, K., et al., $\mathrm{Li}_{x} \mathrm{CoO}_{2}(0<x<-1):$ A new cathode material for batteries of high energy density. Materials Research Bulletin, 1980. 15(6): p. 783-789.

14. $\quad$ Badding, J.V., Cobalt oxide layers. Nature Materials, 2003. 2: p. 208.

15. Koshibae, W., K. Tsutsui, and S. Maekawa, Thermopower in cobalt oxides. Physical Review B, 2000. 62(11): p. 6869-6872.

16. Terasaki, I., et al., Novel thermoelectric properties of complex transition-metal oxides. Dalton Transactions, 2010. 39(4): p. 1005-1011.

17. Woermann, E. and A. Muan, Phase equilibria in the system CaO-cobalt oxide in air. Journal of Inorganic and Nuclear Chemistry, 1970. 32(5): p. 1455-1459.

18. Li, S., et al., Synthesis and Thermoelectric Properties of the New Oxide Materials Ca3${ }_{x} \mathrm{Bi}_{x} \mathrm{Co}_{4} \mathrm{O}_{9+\delta}(0.0<x<0.75)$. Chemistry of Materials, $2000.12(8)$ : p. 2424-2427.

19. $\mathrm{Xu}, \mathrm{G}$., et al., Thermoelectric properties of the $\mathrm{Bi}$ - and $\mathrm{Na}$-substituted $\mathrm{Ca}_{3} \mathrm{Co}_{4} \mathrm{O}_{9}$ system. Applied Physics Letters, 2002. 80(20): p. 3760-3762.

20. Shikano, M. and R. Funahashi, Electrical and thermal properties of single-crystalline $\left(\mathrm{Ca}_{2} \mathrm{CoO}_{3}\right)_{0.7} \mathrm{CoO}_{2}$ with a $\mathrm{Ca}_{3} \mathrm{CO}_{4} \mathrm{O}_{9}$ structure. Applied Physics Letters, 2003. 82(12): p. 1851-1853.

21. Wang, D., et al., Fabrication and thermoelectric properties of $\mathrm{Ca}_{3-} \mathrm{Dy}_{x} \mathrm{Co}_{4} \mathrm{O}_{9+\delta}$ system. Journal of Alloys and Compounds, 2004. 376(1): p. 58-61. 
22. Wang, D., et al., High-temperature thermoelectric properties of $\mathrm{Ca}_{3} \mathrm{Co}_{4} \mathrm{O}_{9+\delta}$ with Eu substitution. Solid State Communications, 2004. 129(9): p. 615-618.

23. Mikami, M., N. Ando, and R. Funahashi, The effect of Ag addition on electrical properties of the thermoelectric compound $\mathrm{Ca}_{3} \mathrm{Co}_{4} \mathrm{O}_{9}$. Journal of Solid State Chemistry, 2005. 178(7): p. 2186-2190.

24. Mikami, M., et al., Bi-Substitution Effects on Crystal Structure and Thermoelectric Properties of $\mathrm{Ca}_{3} \mathrm{Co}_{4} \mathrm{O}_{9}$ Single Crystals. Japanese Journal of Applied Physics, 2006. 45(5A): p. 4131-4136.

25. Liu, H.Q., et al., Effect of $G d$-doping on thermoelectric properties of $\mathrm{Ca}_{3} \mathrm{Co}_{4} \mathrm{O}_{9+\delta}$ ceramics. Journal of Materials Science, 2008. 43(21): p. 6933-6937.

26. Wang, Y., et al., High temperature transport and thermoelectric properties of Agsubstituted $\mathrm{Ca}_{3} \mathrm{Co}_{4} \mathrm{O}_{9+\delta}$ system. Journal of Alloys and Compounds, 2008. 448(1): p. 1-5.

27. Liu, H.Q., et al., Thermoelectric properties of $\mathrm{Ca}_{3-x} \mathrm{Y}_{x} \mathrm{Co}_{4} \mathrm{O}_{9+\delta}$ ceramics. Journal of Physics and Chemistry of Solids, 2009. 70(3): p. 600-603.

28. Wang, Y., et al., Comparison of the high temperature thermoelectric properties for Agdoped and $\mathrm{Ag}$-added $\mathrm{Ca}_{3} \mathrm{Co}_{4} \mathrm{O}_{9}$. Journal of Alloys and Compounds, 2009. 477(1): p. 817821.

29. Nong, N.V., C.J. Liu, and M. Ohtaki, Improvement on the high temperature thermoelectric performance of Ga-doped misfit-layered $\mathrm{Ca}_{3} \mathrm{Co}_{4-x} \mathrm{Ga}_{x} \mathrm{O}_{9+\delta}(x=0,0.05,0.1$, and 0.2). Journal of Alloys and Compounds, 2010. 491(1): p. 53-56.

30. Wang, Y., et al., Enhanced high temperature thermoelectric characteristics of transition metals doped $\mathrm{Ca}_{3} \mathrm{Co}_{4} \mathrm{O}_{9+\delta}$ by cold high-pressure fabrication. Journal of Applied Physics, 2010. 107(3): p. 033708.

31. $\mathrm{Xu}, \mathrm{L} ., \mathrm{F} . \mathrm{Li}$, and $\mathrm{Y}$. Wang, High-temperature transport and thermoelectric properties of $\mathrm{Ca}_{3} \mathrm{Co}_{4-x} \mathrm{Ti}_{x} \mathrm{O}_{9}$. Journal of Alloys and Compounds, 2010. 501(1): p. 115-119.

32. Nong, N.V., C.-J. Liu, and M. Ohtaki, High-temperature thermoelectric properties of late rare earth-doped $\mathrm{Ca}_{3} \mathrm{Co}_{4} \mathrm{O}_{9+\delta}$. Journal of Alloys and Compounds, 2011. 509(3): p. 977981.

33. Pei, J., et al., High temperature transport and thermoelectric properties of $\mathrm{Ca}_{3-x} \mathrm{Er}_{x} \mathrm{Co}_{4} \mathrm{O}_{9+\delta}$. Physica B: Condensed Matter, 2011. 406(3): p. 571-574.

34. Van Nong, N., et al., Enhancement of the Thermoelectric Performance of p-Type Layered Oxide $\mathrm{Ca}_{3} \mathrm{CO}_{4} \mathrm{O}_{9+\delta}$ Through Heavy Doping and Metallic Nanoinclusions. Advanced Materials, 2011. 23(21): p. 2484-2490.

35. Zhang, F.P., et al., Preparation and high temperature thermoelectric properties of $\mathrm{Ca}_{3-x} \mathrm{Ag}_{x} \mathrm{Co}_{4} \mathrm{O}_{9+\delta}$ oxides. Solid State Ionics, 2011. 201(1): p. 1-5.

36. Zhang, F.P., et al., Effects of Pr doping on thermoelectric transport properties of $\mathrm{Ca}_{3-x} \mathrm{Pr}_{x} \mathrm{Co}_{4} \mathrm{O}_{9}$. Solid State Sciences, 2011. 13(7): p. 1443-1447.

37. Butt, S., et al., High-temperature thermoelectric properties of La and Fe co-doped CaCo-O misfit-layered cobaltites consolidated by spark plasma sintering. Journal of Alloys and Compounds, 2014. 588: p. 277-283.

38. Butt, S., et al., Enhancement of thermoelectric performance in $\mathrm{Cd}$-doped $\mathrm{Ca}_{3} \mathrm{Co}_{4} \mathrm{O}_{9}$ via spin entropy, defect chemistry and phonon scattering. Journal of Materials Chemistry A, 2014. 2(45): p. 19479-19487.

39. Prasoetsopha, N., et al., The effect of Cr substitution on the structure and properties of misfit-layered $\mathrm{Ca}_{3} \mathrm{Co}_{4-x} \mathrm{Cr}_{x} \mathrm{O}_{9+\delta}$ thermoelectric oxides. Journal of Alloys and Compounds, 2014. 588: p. 199-205. 
40. Tian, R., et al., Enhancement of high temperature thermoelectric performance in Bi, Fe codoped layered oxide-based material $\mathrm{Ca}_{3} \mathrm{Co}_{4} \mathrm{O}_{9+\delta}$. Journal of Alloys and Compounds, 2014. 615: p. 311-315.

41. Carvillo, P., et al., Thermoelectric Performance Enhancement of Calcium Cobaltite through Barium Grain Boundary Segregation. Inorganic Chemistry, 2015. 54(18): p. 90279032.

42. Gunes, M. and M. Ozenbas, Effect of grain size and porosity on phonon scattering enhancement of $\mathrm{Ca}_{3} \mathrm{Co}_{4} \mathrm{O}_{9}$. Journal of Alloys and Compounds, 2015. 626: p. 360-367.

43. Boyle, C., et al., Grain boundary segregation and thermoelectric performance enhancement of bismuth doped calcium cobaltite. Journal of the European Ceramic Society, 2016. 36(3): p. 601-607.

44. Huang, C.S., et al., Enhanced thermoelectric figure of merit through electrical and thermal transport modulation by dual-doping and texture modulating for $\mathrm{Ca}_{3} \mathrm{Co}_{4} \mathrm{O}_{9+\delta}$ oxide materials. Journal of Alloys and Compounds, 2016. 687: p. 87-94.

45. Presečnik, M., J. de Boor, and S. Bernik, Synthesis of single-phase $\mathrm{Ca}_{3} \mathrm{Co}_{4} \mathrm{O}_{9}$ ceramics and their processing for a microstructure-enhanced thermoelectric performance. Ceramics International, 2016. 42(6): p. 7315-7327.

46. Sotelo, A., et al., Tailoring $\mathrm{Ca}_{3} \mathrm{Co}_{4} \mathrm{O}_{9}$ microstructure and performances using a transient liquid phase sintering additive. Journal of the European Ceramic Society, 2016. 36(4): p. 1025-1032.

47. Bittner, M., et al., Porous $\mathrm{Ca}_{3} \mathrm{CO}_{4} \mathrm{O}_{9}$ with enhanced thermoelectric properties derived from Sol-Gel synthesis. Journal of the European Ceramic Society, 2017. 37(13): p. 3909-3915.

48. Boyle, C., et al., Competing dopants grain boundary segregation and resultant seebeck coefficient and power factor enhancement of thermoelectric calcium cobaltite ceramics. Ceramics International, 2017. 43(14): p. 11523-11528.

49. Porokhin, S., et al., Effect of $\mathrm{NaF}$ doping on the thermoelectric properties of $\mathrm{Ca}_{3} \mathrm{Co}_{4} \mathrm{O}_{9}$ Journal of Alloys and Compounds, 2017. 695: p. 2844-2849.

50. Saini, S., et al., Terbium Ion Doping in $\mathrm{Ca}_{3} \mathrm{Co}_{4} \mathrm{O}_{9}$ : A Step towards High-Performance Thermoelectric Materials. Scientific Reports, 2017. 7: p. 44621.

51. Boyle, C., et al., Improving the thermoelectric performance and thermal stability of $\mathrm{Ca}_{3} \mathrm{CO}_{4} \mathrm{O}_{9+\delta}$ ceramics by sintering in oxygen atmosphere. Journal of Sol-Gel Science and Technology, 2018. 85(3): p. 712-722.

52. Hira, U., et al., High-temperature thermoelectric properties of $\mathrm{Na}$ - and W-Doped $\mathrm{Ca}_{3} \mathrm{Co}_{4} \mathrm{O}_{9}$ system. RSC Advances, 2018. 8(22): p. 12211-12221.

53. Romo-De-La-Cruz, C., et al., Role of oversized dopant potassium on the nanostructure and thermoelectric performance of calcium cobaltite ceramics. Sustainable Energy \& Fuels, 2018. 2(4): p. 876-881.

54. Bittner, M., et al., Triple-phase ceramic $2 D$ nanocomposite with enhanced thermoelectric properties. Journal of the European Ceramic Society, 2019. 39(4): p. 1237-1244.

55. Bittner, M., et al., A comprehensive study on improved power materials for hightemperature thermoelectric generators. Journal of Power Sources, 2019. 410-411: p. 143151.

56. Torres, M.A., et al., Significant enhancement of the thermoelectric performance in $\mathrm{Ca}_{3} \mathrm{Co}_{4} \mathrm{O}_{9}$ thermoelectric materials through combined strontium substitution and hotpressing process. Journal of the European Ceramic Society, 2019. 39(4): p. 1186-1192. 
57. Chen, S., et al., Effect of precursor calcination temperature on the microstructure and thermoelectric properties of $\mathrm{Ca}_{3} \mathrm{Co}_{4} \mathrm{O}_{9}$ ceramics. Journal of Sol-Gel Science and Technology, 2012. 64(3): p. 627-636.

58. Sotelo, A., et al., Improvement of thermoelectric properties of $\mathrm{Ca}_{3} \mathrm{Co}_{4} \mathrm{O}_{9}$ using soft chemistry synthetic methods. Journal of the European Ceramic Society, 2012. 32(10): p. 2415-2422.

59. Delorme, F., et al., Thermoelectric properties of $\mathrm{Ca}_{3} \mathrm{Co}_{4} \mathrm{O}_{9}-\mathrm{Co}_{3} \mathrm{O}_{4}$ composites. Ceramics International, 2015. 41(8): p. 10038-10043.

60. Masset, A.C., et al., Misfit-layered cobaltite with an anisotropic giant magnetoresistance: $\mathrm{Ca}_{3} \mathrm{Co}_{4} \mathrm{O}_{9}$ Physical Review B, 2000. 62(1): p. 166-175.

61. Nagasawa, K., et al., $\mathrm{Ca}_{3} \mathrm{Co}_{4} \mathrm{O}_{9-\delta}$ : A Thermoelectric Material for SOFC Cathode. Chemistry of Materials, 2009. 21(19): p. 4738-4745.

62. Guilmeau, E., et al., Thermoelectric properties-texture relationship in highly oriented $\mathrm{Ca}_{3} \mathrm{Co}_{4} \mathrm{O}_{9}$ composites. Applied Physics Letters, 2004. 85(9): p. 1490-1492.

63. Mikami, M., et al., Enhancement of Electrical Properties of the Thermoelectric Compound $\mathrm{Ca}_{3} \mathrm{Co}_{4} \mathrm{O}_{9}$ through Use of Large-grained Powder. Journal of Materials Research, 2011. 20(9): p. 2491-2497.

64. Zhu, X., et al., Performance evaluation of $\mathrm{Ca}_{3} \mathrm{Co}_{4} \mathrm{O}_{9-\delta}$ cathode on $\mathrm{Sm}_{0.075} \mathrm{Nd}_{0.075} \mathrm{Ce}_{0.85} \mathrm{O}_{2-\delta}$ electrolyte for solid oxide fuel cells. Journal of Alloys and Compounds, 2017. 694: p. 877883.

65. Thoréton, V., et al., Oxygen transport kinetics of the misfit layered oxide $\mathrm{Ca}_{3} \mathrm{Co}_{4} \mathrm{O}_{9+\delta}$. Journal of Materials Chemistry A, 2014. 2(46): p. 19717-19725.

66. $\mathrm{Hu}, \mathrm{Y}$., et al., Oxide diffusion in innovative SOFC cathode materials. Faraday Discussions, 2014. 176(0): p. 31-47.

67. Cutler, M. and N.F. Mott, Observation of Anderson Localization in an Electron Gas. Physical Review, 1969. 181(3): p. 1336-1340.

68. Mehdizadeh Dehkordi, A., et al., Large Thermoelectric Power Factor in Pr-Doped $\mathrm{SrTiO}_{3-\delta}$ Ceramics via Grain-Boundary-Induced Mobility Enhancement. Chemistry of Materials, 2014. 26(7): p. 2478-2485.

69. Zide, J.M.O., et al., Demonstration of electron filtering to increase the Seebeck coefficient

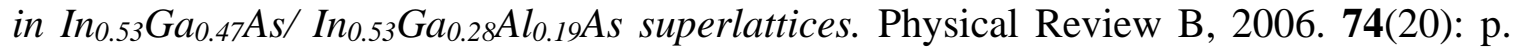
205335.

70. Wang, Y., et al., Doping-Induced Metal-Insulator Transition and the Thermal Transport Properties in $\mathrm{Ca}_{3-x} \mathrm{Y}_{x} \mathrm{Co}_{4} \mathrm{O}_{9}$. The Journal of Physical Chemistry C, 2010. 114(11): p. 51745181.

71. Watari, K., et al., Thermal conductivity of AlN ceramic with a very low amount of grain boundary phase at 4 to $1000 \mathrm{~K}$. Journal of Materials Research, 2011. 17(11): p. 2940-2944.

72. LeBlanc, S., et al., Material and manufacturing cost considerations for thermoelectrics. Renewable and Sustainable Energy Reviews, 2014. 32: p. 313-327.

73. Kim, H.S., et al., Relationship between thermoelectric figure of merit and energy conversion efficiency. Proceedings of the National Academy of Sciences, 2015. 112(27): p. 8205.

74. Lotgering, F. K. Topotactical reactions with ferrimagnetic oxides having hexagonal crystal structures-I. Journal of Inorganic and Nuclear Chemistry, 1959. 9(2): p. 113-123. 


\section{Chapter 10. Conclusion and Future Work}

\section{1 Conclusion}

There are some remaining challenges that need to be studied and explained in order to establish a complete interpretation of single and dual doping influence on the TE properties of polycrystalline $\mathrm{Ca}_{3} \mathrm{Co}_{4} \mathrm{O}_{9}$. Research can be performed to further improve the $\mathrm{ZT}$ performance with value $\sim 0.9$ for polycrystalline $\mathrm{Ca}_{2.95} \mathrm{~Tb}_{0.05} \mathrm{Co}_{4} \mathrm{O}_{9} \mathrm{Bi}_{0.25}$ and further compete with the performance of state-of-the-art $p$-type materials.

For polycrystalline $\mathrm{Ca}_{3} \mathrm{Co}_{4} \mathrm{O}_{9}$ materials, it was shown that one possible approach is to study the effect of dual cationic stoichiometric substitution and non-stoichiometric addition. Such approach could lead to $\rho$ values $\sim 13 \mu \Omega \mathrm{m}$ which are up to $70 \%$ better than the pristine material values and they are reached by improving the crystal texture (and carrier mobility) with simple chemistry modification. Additionally, introduction of more extrinsic heavy dopants may reduce $\kappa$ (by increasing the phonon scattering) of the polycrystalline $\mathrm{Ca}_{3} \mathrm{Co}_{4} \mathrm{O}_{9}$ up to $25 \%$, such is the case for the extremely low $\kappa \sim 1.28 \mathrm{~W} / \mathrm{mK}$ for $\mathrm{Ca}_{2.5} \mathrm{~Tb}_{0.5} \mathrm{Co}_{4} \mathrm{O}_{9}$. Moreover, modulation of the GB interfaces could increase phonon scattering at neighboring crystals, and hence more scattering compared to the case of single element doping. Chapter 9 shows that the high ZT is tunable in terms of dopant species and level, such is the case for the high ZT $\sim 0.9$ at $973 \mathrm{~K}$ for $\mathrm{Ca}_{3} \mathrm{Co}_{4} \mathrm{O}_{9} \mathrm{Bi}_{0.25}$ and the high $\mathrm{ZT} \sim 0.82$ at $1073 \mathrm{~K}$ for $\mathrm{Ca}_{2.94} \mathrm{~Tb}_{0.03} \mathrm{Pr}_{0.03} \mathrm{Co}_{4} \mathrm{O}_{9} \mathrm{Bi}_{0.24}$.

Furthermore, it is useful to study the effect of each single dopant to understand the specific influence on the TE properties. In this approach, one may tune the total $\kappa$ with the introduction of heavier atoms and grain boundary modulation, find the optimum condition for the lowest $\rho$ with the improved crystal texture by the chemistry modification, and at the same time improve $S$ (by 
reducing carrier concentration) with carrier filtering mechanisms at grain boundaries by the segregation of dopants or with the use of high valence state elements. Table 10-1 provides a more detail information about the aforementioned effects and peak improvements by each concentration analyzed in this work.

Table 10-1: Summary of peak improvements for different analyzed materials by chemistry modification $\mathrm{Ca}_{3} \mathrm{Co}_{4} \mathrm{O}_{9}$

\begin{tabular}{|c|c|c|}
\hline Bulk chemistry & Improved value & Relevant properties notes \\
\hline $\mathrm{Ca}_{3} \mathrm{Co}_{4} \mathrm{O}_{9} \mathrm{~K}_{0.10}$ & $\begin{array}{l}\boldsymbol{\rho}=27.6 \mu \Omega \mathrm{m}(38 \% \text { reduction }) \text { at LT } \\
\boldsymbol{\kappa}=2.08 \mathrm{~W} / \mathrm{mK}(25 \% \text { increase }) \text { at HT } \\
\boldsymbol{S}=160 \mu \mathrm{VK}^{-1}(18 \% \text { increase }) \text { at LT } \\
\boldsymbol{Z T}=0.42(20 \% \text { increase }) \text { at HT }\end{array}$ & $\begin{array}{l}\boldsymbol{\sigma} \boldsymbol{S}^{2} \text { increased } 50 \% \text { from } \\
\text { improvements in crystal } \\
\text { texture and alignment. } \\
\text { Negatively high } \boldsymbol{\kappa} \text { from light } \\
\text { weight of } \mathrm{K}^{+} \text {. }\end{array}$ \\
\hline $\mathrm{Ca}_{3} \mathrm{Co}_{4} \mathrm{O}_{9} \mathrm{Bi}_{0.15} \mathrm{~K}_{0.05}$ & $\begin{array}{l}\boldsymbol{\rho}=27.7 \mu \Omega \mathrm{m}(38 \% \text { reduction }) \text { at } \mathrm{LT} \\
\boldsymbol{\kappa}=1.85 \mathrm{~W} / \mathrm{mK}(11 \% \text { increase }) \text { at } \mathrm{HT} \\
\boldsymbol{S}=138 \mu \mathrm{VK}^{-1}(1 \% \text { increase }) \text { at } \mathrm{LT} \\
\boldsymbol{Z T}=0.44(30 \% \text { increase }) \text { at HT }\end{array}$ & $\begin{array}{l}\sigma \boldsymbol{S}^{2} \text { increased } 40 \% \text { from } \\
\text { increased carrier mobility and } \\
\text { decrease in carrier } \\
\text { concentration. Incorporation } \\
\text { of Bi does not overcome high } \\
\boldsymbol{\kappa} \text { coming from addition of } \mathrm{K}^{+} \text {. }\end{array}$ \\
\hline $\mathrm{Ca}_{2.95} \mathrm{~Tb}_{0.05} \mathrm{Co}_{4} \mathrm{O}_{9}$ & $\begin{array}{l}\boldsymbol{\rho}=64.2 \mu \Omega \mathrm{m}(3 \% \text { reduction }) \text { at } \mathrm{HT} \\
\boldsymbol{\kappa}=1.6 \mathrm{~W} / \mathrm{mK}(4 \% \text { reduction }) \text { at } \mathrm{HT} \\
\boldsymbol{S}=156 \mu \mathrm{VK}^{-1}(15 \% \text { increase }) \text { at } \mathrm{LT} \\
\boldsymbol{Z T}=0.38(10 \% \text { increase }) \text { at } \mathrm{HT}\end{array}$ & $\begin{array}{l}\text { Intensified phonon scattering } \\
\text { events from increased GB } \\
\text { density. Deterioration of } \\
\text { electrical transport properties } \\
\text { from inferior crystal texture. }\end{array}$ \\
\hline $\mathrm{Ca}_{2.95} \mathrm{~Tb}_{0.05} \mathrm{Co}_{4} \mathrm{O}_{9} \mathrm{~K}_{0.1}$ & $\begin{array}{l}\boldsymbol{\rho}=47.7 \mu \Omega \mathrm{m}(28 \% \text { reduction }) \text { at } \mathrm{HT} \\
\boldsymbol{\kappa}=1.85 \mathrm{~W} / \mathrm{mK}(11 \% \text { increase }) \text { at } \mathrm{HT} \\
\boldsymbol{S}=165 \mu \mathrm{VK}^{-1}(23 \% \text { increase }) \text { at } \mathrm{LT} \\
\boldsymbol{Z T}=0.47(34 \% \text { increase }) \text { at HT }\end{array}$ & $\begin{array}{l}\text { Tb help to provide better } \\
\text { thermal properties while } \mathrm{K}^{+} \\
\text {offer better electrical } \\
\text { properties. GB filtering } \\
\text { influence a big increase in } S \text {. }\end{array}$ \\
\hline $\mathrm{Ca}_{2.95} \mathrm{~Tb}_{0.05} \mathrm{Co}_{4} \mathrm{O}_{9} \mathrm{Bi}_{0.25}$ & $\begin{array}{l}\boldsymbol{\rho}=13.3 \mu \Omega \mathrm{m}(70 \% \text { reduction }) \text { at } \mathrm{LT} \\
\boldsymbol{\kappa}=1.48 \mathrm{~W} / \mathrm{mK}(12 \% \text { reduction }) \text { at } \mathrm{HT} \\
\boldsymbol{S}=156 \mu \mathrm{VK}^{-\boldsymbol{1}}(16 \% \text { increase }) \text { at } \mathrm{LT} \\
\boldsymbol{Z T}=\mathbf{0 . 9 0}(\mathbf{2 . 6 x} \text { increase }) \text { at } \mathrm{HT}\end{array}$ & $\begin{array}{l}\text { Nanostructure control of GB } \\
\text { facilitate a better crystal } \\
\text { texture with improved carrier } \\
\text { mobility and electrical } \\
\text { transport properties } 4.5 \text { times } \\
\text { better than baseline. }\end{array}$ \\
\hline
\end{tabular}

*Optimal parameter requirement: $\boldsymbol{\rho}$ reduction, $\boldsymbol{\kappa}$ reduction, $\boldsymbol{S}$ increase, and $\boldsymbol{Z T}$ increase. HT=High temperature1073 K; LT=Low temperature $\sim 373 \mathrm{~K}$. 
Given the complex nature of the strongly correlated TE properties for polycrystalline $\mathrm{Ca}_{3} \mathrm{Co}_{4} \mathrm{O}_{9}$ it will remain a challenge to achieve a $\mathrm{ZT}$ higher than 0.9 , nonetheless, those fundamental limitations could be overcome with specific approaches to control separately the electronic and thermal transport properties. Employing the above perspectives in polycrystalline $\mathrm{Ca}_{3} \mathrm{Co}_{4} \mathrm{O}_{9} \mathrm{TE}$ research requires the understanding of the suitable elements to properly control all the transport properties needed for a high ZT.

Thus, to further investigate the influence in dual dopants on the TE performance and the nanostructure properties of the polycrystalline $\mathrm{Ca}_{3} \mathrm{Co}_{4} \mathrm{O}_{9}$. It is of big importance to understand first the effect of single dopants to be able to use them in a systematic dual doping approach. Therefore, future work needs to center around already studied single-doping elements for $\mathrm{Ca}_{3} \mathrm{Co}_{4} \mathrm{O}_{9}$ to be able precisely modify the crystal texture, the grain boundary, the carrier concentration, the carrier mobility, and the phonon scattering events. Moreover, a second dopant from the pool of existing knowledge could be selected to tackle the TE limitations after the introduction of the first dopant, thus, giving a key boost to expand the performance of the single doping for $\mathrm{Ca}_{3} \mathrm{Co}_{4} \mathrm{O}_{9}$.

In this work we focus on both the conventional stoichiometric substitution and in the novel non-stoichiometric addition approach to improve the thermoelectric performance of $\mathrm{Ca}_{3} \mathrm{Co}_{4} \mathrm{O}_{9-\delta}$ ceramics. This versatile approach to introduce dopants in the bulk material resulted in and improvement of $260 \%$ on the TE performance by controlling the intragranular and concurrent intergranular segregation/depletion of the doping element at the grain boundaries as shown in Figure 10-1. Remarkably, dopant segregation promoted the crystal texture and alignment in $\mathrm{Ca}_{3} \mathrm{Co}_{4} \mathrm{O}_{9-\delta}$ which facilitate an improved carrier mobility and electrical transport properties almost 4.5 times better compared to baseline. Crystal growth is a direct consequence of a faster diffusion due to the segregated dopants at the GB. In addition, the layer of doping elements segregated at 
the GB works as carrier filter to decrease the carrier concentration and simultaneously increase the $S$ to a value of $156 \mu \mathrm{VK}^{-1}$ (16\% increase). Moreover, the inclusion of the heavy atomic weight of doping elements could provide with phonon scattering events leading to a thermal conductivity values of $1.48 \mathrm{~W} / \mathrm{mK}\left(12 \%\right.$ reduction)at $1073 \mathrm{~K}$. The modification on polycrystalline $\mathrm{Ca}_{3} \mathrm{Co}_{4} \mathrm{O}_{9-\delta}$ ultimately resulted in a world high record performance $\mathrm{ZT} \sim 0.9$ and in the systematic microstructure evolution as seen in Figure 10-1.
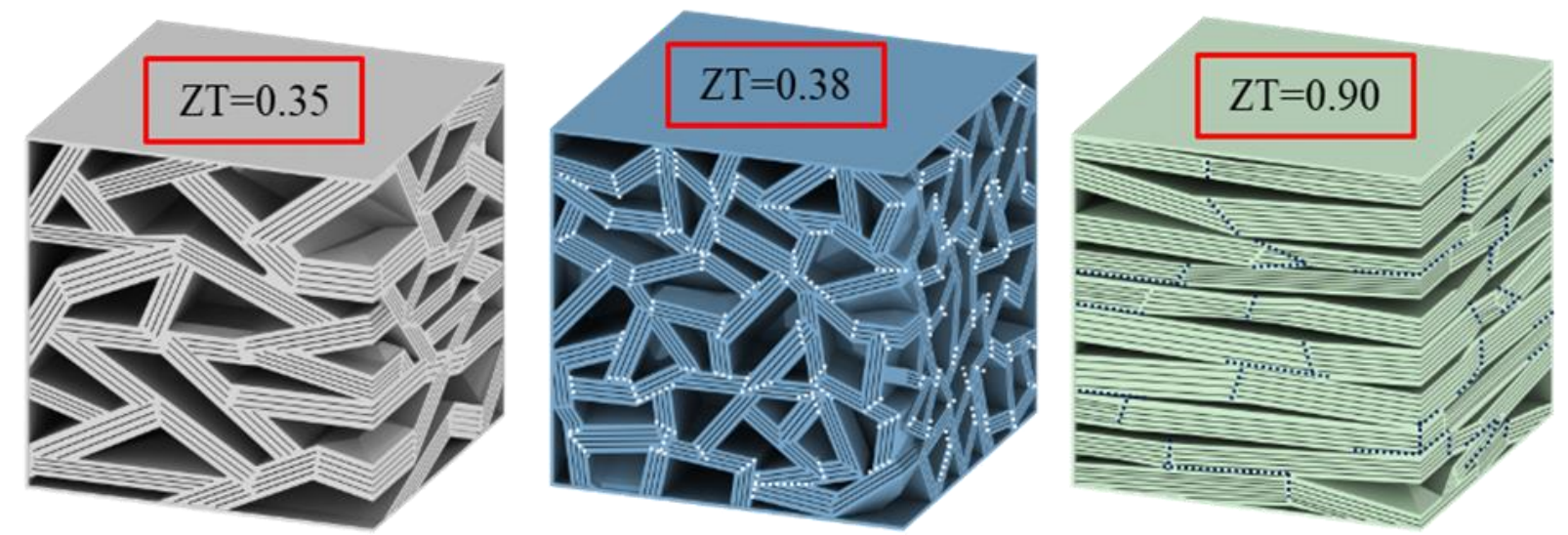

Figure 10-1: Schematic showing the systematic microstructure evolution of $\mathrm{Ca}_{3} \mathrm{Co}_{4} \mathrm{O}_{9}$.

\section{2 Future work}

Encouraged by the high ZT achieved in this work, certain improvements and future work are recommended for the implementation of this research into a viable thermoelectric generator. Although many aspects of the experimental setup used to test the thermoelectric properties are reliable, the thermoelectric power unit tests have several areas for improvement. First, the electrical properties were measured in a low pressure He environment due to limitations of the experimental setup which is not the real environment where the material will be working, thus, further characterization in air is desired. In addition to the characterization in air, is far more critical to obtain the power density and power output of the actual TE modules at different operating temperature for the power unit. This would allow to better characterize the material systems and 
provide a more precise performance for commercial applications. In addition, research related to the contact resistance and materials utilized to bond the multiple $p$ and $n$-type legs is also required. Additionally, it is highly desired to scale up the TE devices from the small-scale experimentation. A large-scale TE device for the recovery of waste heat, as those commercially available, with multiple $\mathrm{n}$ and $p$-type legs allowing useful power generation would be worthwhile exploring in the near future with the ZT performance achieved for the bulk $\mathrm{Ca}_{3} \mathrm{Co}_{4} \mathrm{O}_{9}$ in this work.
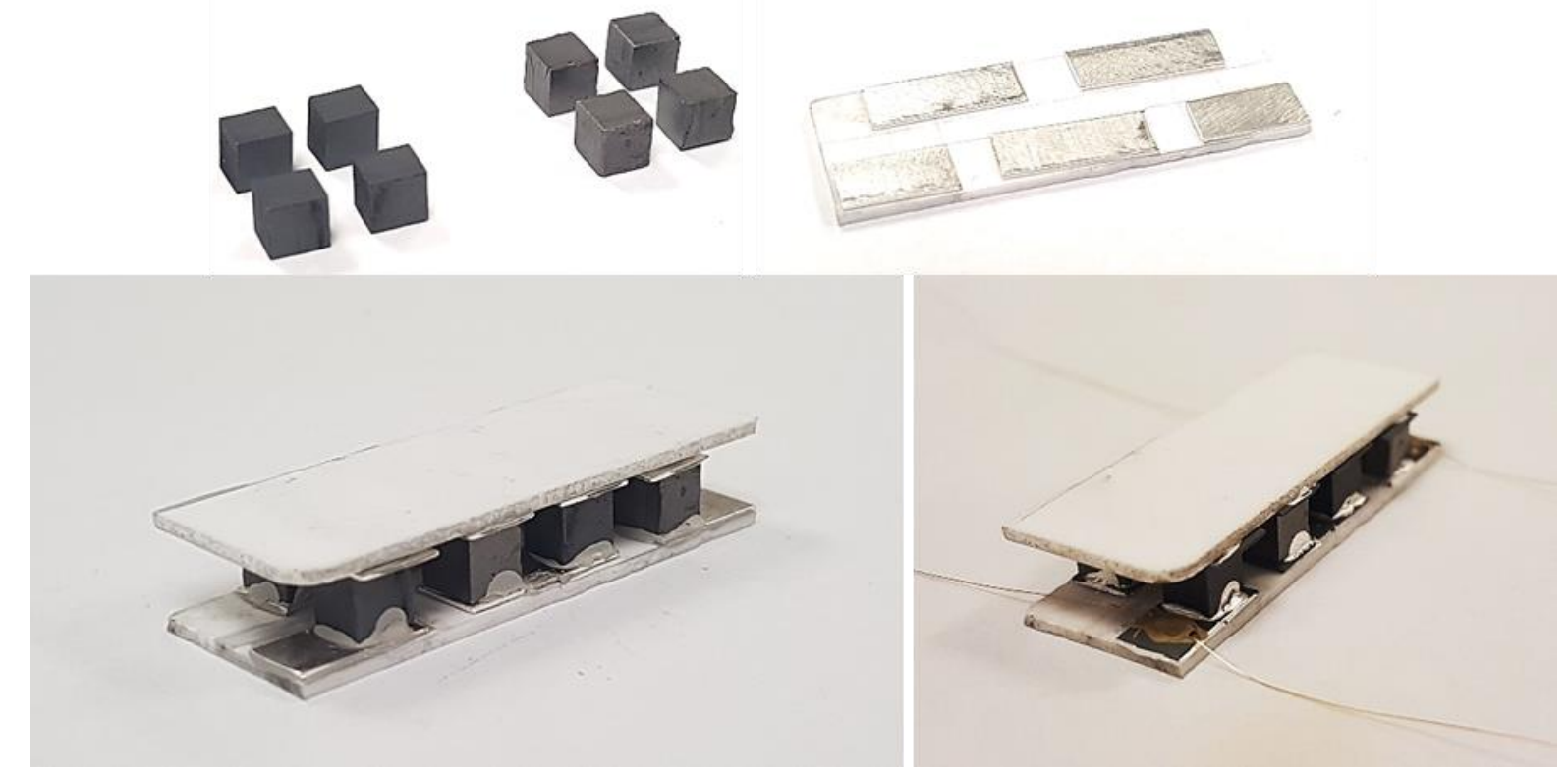

Figure 10-2: Multiple leg setup for prototype of $\mathrm{Ca}_{3} \mathrm{Co}_{4} \mathrm{O}_{9}$ legs as p-type material and $\mathrm{CaMnO}_{3}$ as n-type material using silver paste for bonding the legs with the silver foil and alumina.

To provide some early experiments in this aspect, two unicouples consisting of various $\mathrm{Ca}_{3} \mathrm{Co}_{4} \mathrm{O}_{9}$ legs as $p$-type material (from both the baseline and the best performing material in this work) and $\mathrm{CaMnO}_{3}$ as $n$-type material (from both a baseline and utilizing a previous reported material from Song et al.) was made as shown in Figure 10-2. To make the TE module each pellet was sinter separate and cut it in same height and width. Then, colloidal silver paste was used to glue the silver foil to the alumina and was also used to glue silver foil to each leg. Once the TE module set up was complete, the device was fired at $973 \mathrm{~K}$ for 1 hour to bond the silver paste to the legs, alumina, and silver foil. Gold wires connections were added to perform the measurements. 
The hot side of the thermoelectric module was at the restrictive high temperature of the hot plate at $800 \mathrm{~K}$ while water flow was utilized to keep the cold side below $373 \mathrm{~K}$. Measurements were done using a Gamry Reference 3000 w/ AE potentiostat.
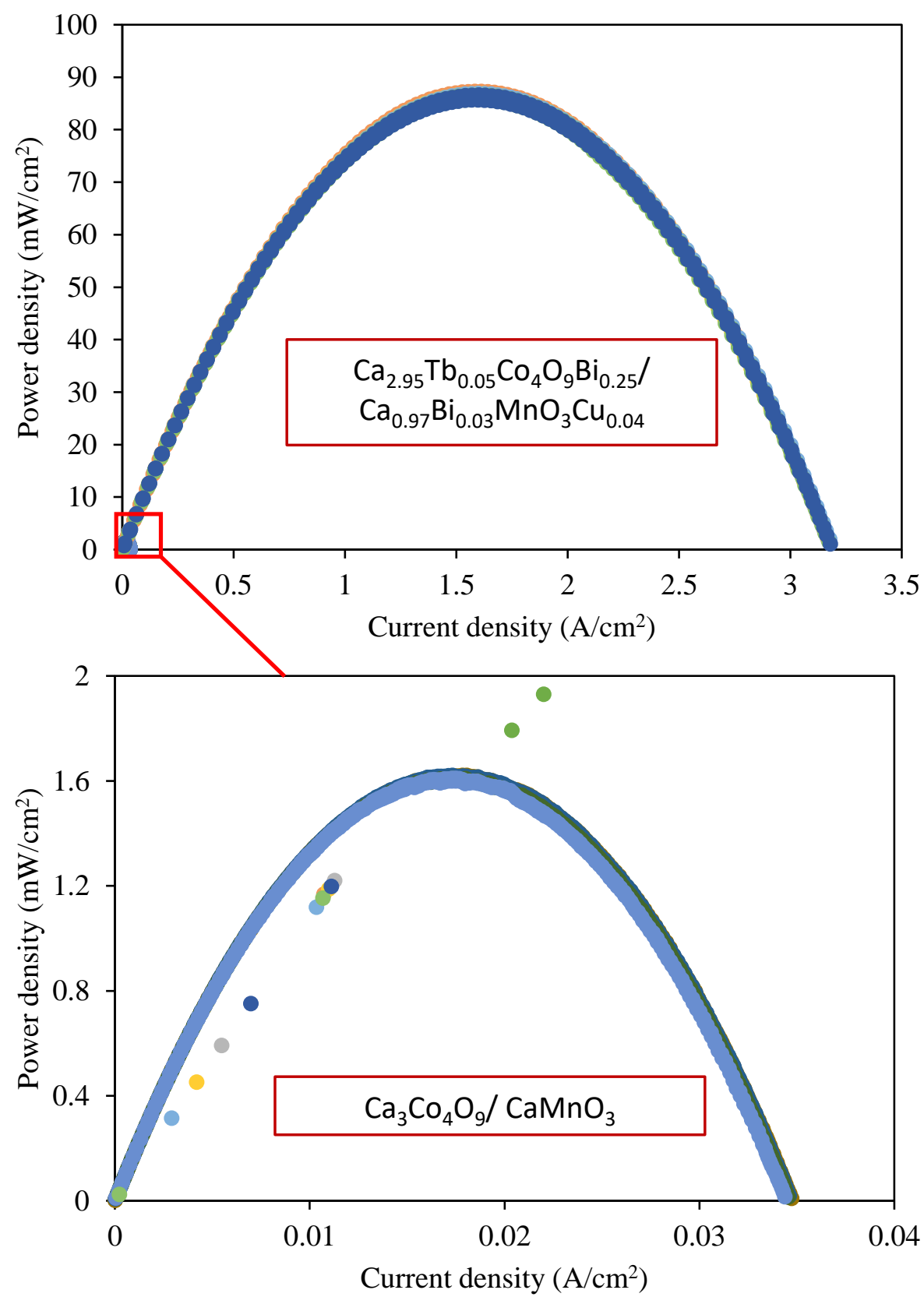

Figure 10-3: Characteristic of TE modules as function of the electrical current. The electrical power densities were calculated from the active area of the unicouples. 
The power density results of the TE modules for both the baseline and the best performing material are show in Figure 10-3. The power density for the best performing material $\mathrm{Ca}_{2.95} \mathrm{~Tb}_{0.05} \mathrm{Co}_{4} \mathrm{O}_{9} \mathrm{Bi}_{0.25} / \mathrm{Ca}_{0.97} \mathrm{Bi}_{0.03} \mathrm{MnO}_{3} \mathrm{Cu}_{0.04}$ is $\sim 90 \mathrm{~mW} / \mathrm{cm}^{2}$, such value represents a power density 55 times larger than that of the unicouples made of baseline materials. The obtained performance is within range of the performance of other reported $\mathrm{Ca}_{3} \mathrm{Co}_{4} \mathrm{O}_{9}$ (Bittner et al.), however those results are reported at temperatures at least $300 \mathrm{~K}$ higher. Thus, these results are encouraging and confirm that the utilization of $\mathrm{Ca}_{2.95} \mathrm{~Tb}_{0.05} \mathrm{Co}_{4} \mathrm{O}_{9} \mathrm{Bi}_{0.25}$ could potentially outperform the best reported TE modules made of $\mathrm{Ca}_{3} \mathrm{Co}_{4} \mathrm{O}_{9}$ ceramics. 


\section{Chapter 11. Publications}

\section{Patent}

- Xueyan S., Romo-De-La-Cruz C., Chen Y., "Significant Improvement in Thermoelectric Performances of Complex Oxide Polycrystalline Ceramics by Designing Grain Boundaries” U.S. Provisional Patent (filed September 2019)

\section{Papers published}

- Romo-De-La-Cruz C., Liang L., Navia S.A.P., Chen Y., Prucz J., Song X., Role of oversized dopant potassium on the nanostructure and thermoelectric performance of calcium cobaltite ceramics. Sustainable Energy \& Fuels. 2018;2(4):876-81.

- Boyle C., Liang L., Romo-De-La-Cruz C., Johnson R., Chen Y., Prucz J., Cakmak E., Watkins T. R., Lara-Curzio E., Song X., Improving the thermoelectric performance and thermal stability of $\mathrm{Ca}_{3} \mathrm{Co}_{4} \mathrm{O}_{9+\delta}$ ceramics by sintering in oxygen atmosphere. Journal of SolGel Science and Technology. 2018;85(3):712-22.

- Xueyan S., Paredes Navia S. A., Liang L., Boyle C., Romo-De-La-Cruz C., Jackson B., Hinerman A., Wilt M., Prucz J., Chen Y., Grain Boundary Phase Segregation for Dramatic Improvement of the Thermoelectric Performance of Oxide Ceramics, ACS Applied Materials \& Interfaces. 2018;10(45): 39018-39024.

- Liang L., Romo-De-La-Cruz C., Carvilo P., Jackson B., Gemmen E., Paredes Navia S. A., Prucz J., Chen Y., Xueyan S., Difference between Transition Metal Cation Substitution and Nonstoichiometric Addition on Nanostructure and Thermoelectric Performance of Complex Oxide Ceramics, Journal of Solid State Chemistry. 2019; 277: 427-433 


\section{Papers to be submitted}

- Romo-De-La-Cruz C., et al., Significant Thermoelectric Performance Improvement Through Manipulating Dopants Distribution in Lattice and Segregation at Grain Boundary Dopants, Nature Energy (to be submitted).

- Romo-De-La-Cruz C., et al., Influence of the Dual Non-Stoichiometric Addition of Bismuth and Potassium in Thermoelectric Performance of Calcium Cobaltite Ceramics, To be submitted.

- Romo-De-La-Cruz C., et al., Enhancement of the Thermoelectric Performance of Calcium Cobaltite Ceramics due to Grain Boundary Segregation of Bismuth Via Non-Stoichiometric Addition, To be submitted.

- Romo-De-La-Cruz C., et al., Review on Improvement of Calcium Cobaltite Ceramics with Conventional Processing and Chemistry Modification, To be submitted. 